\title{
The British Thoracic Society's winter 1994 meeting was held on 12-14 December 1994 at the Queen Elizabeth II Conference Centre, Westminster, London
}

Spatial mapping to relate respiratory morbidity to a point source of air pollution

P MILLIGAN, YJ KELLY, BJ BRABIN, J REID, E DUNNE, MG PEARSON School of Tropical Medicine, Sefton Health, Sefton MBC and Aintree Chest Centre, Liverpool Previously we reported a $60 \%$ increase in excess cough and significantly increased absences from school due to respiratory symptoms, in an area of increased dust pollution in a survey of 1872 children aged 5-11 years in Merseyside. We extended the analysis to relate levels of airborne dust pollution, respiratory symptoms and distance of residence from a point source of air pollution. Residential locations were mapped using postcode data. Airborne dust pollution was monitored for 12 months using dust deposition gauges in the Liverpool dock area and two other areas of the city thought to be typical of urban pollution. Mean dust deposition levels decrease radially as distance from the dock area increases; $0.5 \mathrm{~km}, 115 \mathrm{mg} / \mathrm{m}^{2} /$ day; $1.0 \mathrm{~km}, 90 \mathrm{mg} / \mathrm{m}^{2} /$ day $; 2.0 \mathrm{~km}, 40 \mathrm{mg} / \mathrm{m}^{2} /$ day. Children living within a $1 \mathrm{~km}$ radius of the dock area have a twofold increased risk of respiratory symptoms than those $3 \mathrm{~km}$ from the dock (OR $2 \cdot 1,95 \% \mathrm{CI} 1.5$ to 3.0 ) after adjusting for other environmental, predisposition and socioeconomic risk factors. For children within $1 \mathrm{~km} 35 \%$ had excess cough and $24 \%$ breathlessness against $18 \%$ and $11 \%$ respectively more than $2 \mathrm{~km}$ away. The control areas had $16 \%$ and $19 \%$ excess cough respectively. There is a relationship $(p<0.05)$ between mean dust pollution level in the vicinity of the child's residence and the risk of having excess cough which halved at a distance $2 \mathrm{~km}$ from the dock. The technique of spatial mapping can help identify a probable point source of pollution even in a cross sectional study.

Aeromycology of hospital buildings: non-construction related aspergillosis and detection of contaminated aerosols

G MORRIS, H KENNEDY, I BUTCHER, G SHANKLAND, M RICHARDSON, $K$ ANDERSON Departments of Respiratory Medicine, Medical Mycology, and Bacteriology, Western Infirmary, Glasgow and Public Health, University of Glasgow Nosocomial aspergillosis has been generally associated with building construction in close proximity to immunosuppressed patients. While this observation is likely to be correct for some infection episodes, large hospital sites usually have a continuous programme of building alteration, and there is recent evidence to suggest that building demolition or construction is not always followed by measurable differences in aeromycology (Goodley et al. $\mathcal{F}$ Hosp Infect 1994). We recently investigated two separate nosocomial outbreaks of aspergillosis which developed in a paediatric oncology ward (five cases over six months) and adult haematology ward (seven cases over nine months), neither associated with a discrete spell of construction or demolition. Although both units were served by filtered air conditioning, non-filtered air could enter through windows or the hospital waste disposal conduit to the basement because of the multistorey building design. A fumigatus was isolated from pigeon excreta close to the windows in the adult unit, and was also consistently isolated in air and swab samples around the waste conduits in both units (as well as $A$ niger, flavus, terreus, versicolor and other species - predominately Penicillium). A fumigatus was isolated within the paediatric unit vacuum cleaner and in its exhaust (65 colony forming units $/ \mathrm{m}^{3} v 0-6 \mathrm{cfu} / \mathrm{m}^{3}$ background) which possibly acted as a biological trap and disseminator. The adult unit vacuum cleaner was also contaminated but this reflected the ward predominant airborne organism of Penicillium species. Both outbreaks have resolved after sealing of the disposal conduits and removal of the contaminated vacuum cleaner. Neither of these routes have been reported as a source of nosocomial aspergillosis previously and underscore the importance of all sources of air and aerosols close to this group of patients.

Employment status and the perceived association of occupation with respiratory symptoms in young adults with and without asthma

D JARVIS, P BURNEY, $S$ CHINN, C LUCZYNSKA, E LAI, R HALL, B HARRISON, J STARK Department of Public Health Medicine, UMDS; Department of Respiratory Medicine, The Ipswich Hospital; Department of Respiratory Medicine, West Norwich Hospital; Department of Respiratory Medicine, Addenbrookes Hospital, Cambridge As part of the British arm of The European Community Respiratory Health Survey data on respiratory symptoms and occupational history were collected from a random sample of young adults living in East Anglia. Subjects were considered to have asthma (ast) if they reported being woken by shortness of breath in the last 12 months or asthma attacks in the last 12 months

\begin{tabular}{|c|c|c|c|c|}
\hline & \multicolumn{4}{|c|}{ Current employment status (\%) } \\
\hline & Student & Employed & Unemployed & Housewife \\
\hline \multirow{2}{*}{$\begin{array}{l}\text { Asthma } \\
(n=416) \\
\text { No asthma } \\
(n=1007)\end{array}$} & $3 \cdot 6$ & $73 \cdot 8$ & 11.8 & $10 \cdot 8$ \\
\hline & 3.3 & $80 \cdot 4$ & & $\begin{array}{l}9 \cdot 8 \\
N o \\
\text { asthma }\end{array}$ \\
\hline \multicolumn{3}{|c|}{$\begin{array}{l}\text { Being at work makes chest tight or wheezy } \\
\text { Ever exposed to vapours, dust, gas, fumes at work } \\
\text { Changed job because affected breathing }\end{array}$} & $\begin{array}{r}33 \cdot 2 \% \\
55 \cdot 3 \% \\
8 \cdot 3 \%\end{array}$ & $\begin{array}{r}8 \cdot 6 \% \\
45 \cdot 4 \% \\
2 \cdot 5 \%\end{array}$ \\
\hline
\end{tabular}

or current use of asthma medication. A wide range of occupations were associated with respiratory symptoms at work, perceived exposure to vapours, dust, gas or fumes and changing of occupation because current job affected breathing. Young adults with asthma living in East Anglia report more unemployment than those who do not have asthma. Of those who are employed one third report that 'being at work makes their chest tight or wheezy'. [This work was supported by the National Asthma Campaign.]

Respiratory symptoms and asthma in a working population and in the general population

SC STENTON, JR BEACH, G DEVEREUX, SJ BOURKE, DJ HENDRICK Chest Unit and Regional Unit for Occupational Lung Disease, Nerwcastle General Hospital, University of Newcastle upon Tyne We recently performed two investigations of asthma prevalence involving 876 subjects selected at random from FHSA registers (20-44 years, 289 women) and 1126 shipyard workers (16-25 years, 50 women). All subjects completed a respiratory questionnaire and underwent measurements of airway responsiveness as $\mathrm{PD}_{20} \mathrm{FEV}_{1}$ to methacholine. The distribution of $\mathrm{PD}_{20}$ measurements was similar among the two populations as was the proportion in each $\mathrm{PD}_{20}$ category with a physician-made diagnosis of asthma (table 1). The overall prevalence of asthma symptoms was higher among the general population than among the workers. The general population sample also showed a close association between 
Table 1

\begin{tabular}{lllll}
\hline & $P D_{20}(\mu g$ methacholine $)$ & \\
\cline { 2 - 5 } & 200 & $200-1000$ & $1000-6400$ & $>6400$ \\
\hline General population & 74 & 109 & 117 & 556 \\
$\quad$ n & 73 & 18 & 12 & 4 \\
$\quad$ \% asthmatic & 86 & 184 & 241 & 615 \\
Workers & 62 & 16 & 9 & 2 \\
$\quad$ n & & & & \\
\hline
\end{tabular}

Table 2

\begin{tabular}{lllll}
\hline & \multicolumn{4}{l}{$P D_{20}(\mu g$ methacholine $)$} \\
\cline { 2 - 5 } & 200 & $200-1000$ & $1000-6400$ & $>6400$ \\
\hline General population & 95 & 65 & 48 & 32 \\
Wheeze (\%) & 81 & 45 & 43 & 26 \\
$\quad$ Chest tightness (\%) & & & & \\
Workers & 16 & 23 & 21 & 19 \\
$\quad$ Wheeze (\%) & 12 & 19 & 17 & 16 \\
Chest tightness (\%) & & & & \\
\hline
\end{tabular}

symptom prevalence and $\mathrm{PD}_{20}$ category, but the workers showed little difference in symptom prevalence with changing $\mathrm{PD}_{20}$ (table 2). The questionnaire was thus a poorer guide to the presence of asthma among the workers than among the general population. This might have been due to underreporting of symptoms by some workers worried about job security, and overreporting by others concerned about occupational exposures. Similar biases could distort the results of asthma questionnaires under other circumstances.

\section{Occupational asthma in East London}

SK MEREDITH AND P CULLINAN Department of Epidemiology and Medical Statistics, London Hospital Medical College at QMW, London; Department of Occupational and Environmental Medicine, National Heart and Lung Institute, London To assess the aetiological role of occupation in asthma, a postal survey was undertaken of all asthmatics, 16-64 years old, registered with seven general practices in East London (1430 persons). The questionnaire requested the age of onset (or exacerbation) of asthma symptoms and detailed occupational and smoking histories; home visits were conducted on a $20 \%$ random sample of non-responders. A net response of $72 \%$ was obtained. The prevalence of registered asthma was $4.4 \%$, slightly higher in women than men, with little difference by age. Half of the men (49\%) and $64 \%$ of the women reported onset of asthma after the age of 16 years; of those with childhood onset, $41 \%$ reported a worsening in adulthood ( $35 \%$ of men, $47 \%$ of women). A case-control analysis was carried out of the occupations at the time symptoms began for the 658 persons who reported an onset or exacerbation of asthma after leaving school. Cases $(n=186)$ were defined as those whose asthma began $<2$ years after starting a new job and controls as those whose asthma began while in work, but $\geqslant 2$ years from starting a job. High risk occupations were defined as those with at least five times greater risk of occupational asthma than the general working population (SWORD data). No association was found in women between starting a high risk job and onset of symptoms. In men the odds ratio (OR) was $1 \cdot 2$ ( $95 \%$ CI 0.5 to 3.3 ) overall, but was higher in men under the age of 45 years $(1 \cdot 9)$ and in smokers $(1 \cdot 7)$. The increased risk was confined to those with childhood onset and exacerbation in adulthood (OR 5.3). The attributable proportion derived from these data suggest that about $2 \%$ of asthma in working men in East London is associated with high risk work.

\section{An in vitro biological effect of an antibody to an epithelial cell} autoantigen in cryptogenic fibrosing alveolitis (CFA)

wah wallace, D Lamb, Sem howie Department of Pathology, Edinburgh University Medical School, Teviot Place, Edinburgh EH8 $9 A G$ We have previously described the presence of circulating IgG autoantibodies to an extractable lung antigen of $70-90 \mathrm{kDa}$ (Thorax 1994;49:218-24) in patients with CFA. Using serum obtained by immunisation of a rabbit with the partially purified antigen we have demonstrated that it appears to be lung specific, associated with alveolar epithelial lining cells, and present in the A549 type II epithelial cell line (Thorax 1994;49:1139-45). In order to assess whether this antibody might have biological activity we cultured A549 cells and a pulmonary fibroblast cell line in medium containing a range of concentrations $(0.001-10 \%)$ of immune or control rabbit serum for 72 hours in 96 well plates. The number of cells in each well at the end of this period was assessed using an MTT assay ( $\mathcal{F}$ Immunol Methods 1983;65:55-63). The results indicated that the immune rabbit serum at concentrations between $3 \%$ and $0.1 \%$ resulted in significantly fewer A549 cells after 72 hours than the presence of identical concentrations of control rabbit serum $(3 \% \mathrm{p}<0.05,1 \%$ $\mathrm{p}<0.01,0.3 \% \mathrm{p}<0.001,0.1 \% \mathrm{p}<0.05)$. At concentrations lower than this no differential effect was observed. Identical experiments using the fibroblasts showed no such effect. Further experiments conducted using purified control and immune rabbit IgG in the presence of fresh and heat inactivated rabbit serum confirmed that the effect on the A549 cells was IgG and complement mediated. The rabbit antiserum directed against the putative $70-90 \mathrm{kDa}$ antigen in CFA appears to have in vitro biological activity against the A549 cell line which is IgG and complement mediated. It is not clear whether the effects observed are due to complement mediated cell death or a reduction in cell proliferation. These results suggest a possible biological role for the autoantibody identified in patients with CFA which may have significance in the pathogenesis of the disease.

Epstein-Barr virus (EBV) in type II pneumocytes in cryptogenic fibrosing alveolitis

JJ EGAN, JP STEWART, PS HASLETON, JR ARRAND, KB CARROLL, AA woodcock North West Lung Centre, Department of Histopathology, Wythenshawe Hospital, Manchester; Department of Molecular Biology, Paterson Institute for Cancer Research, Christie Hospital Manchester Cryptogenic fibrosing alveolitis (CFA) is a clinically heterogeneous condition. Both environmental and infective factors have been implicated in its aetiology. We have investigated surgically obtained lung tissue from $20 \mathrm{CFA}$ patients $(17$ men, mean age 58 years, range 39-69) for evidence of EBV replication and compared this with lung tissue of 21 control patients $(n=21,14$ men, mean age 61 years, range 32-75). Only patients with a typical histological and clinical picture were designated CFA. Fourteen of the 20 CFA patients had received no specific therapy for CFA at the time of biopsy. Monoclonal antibodies directed against the EBV viral antigens, EBV viral capsid antigen (VCA, MAB 817; Chemicon International Temecula, California, USA) and gp340/220 antigen, which are expressed during the lytic (replicating) phase of the EBV life cycle, were studied. Expression of viral and cellular markers within the tissue blocks were analysed by immunofluorescence using a streptavidinbiotin amplification step. Fourteen of $20(70 \%)$ of the CFA patients were positive for both EBV VCA and gp340/220 compared with two of $21(9 \%)$ of the control group $\left(\chi^{2}\right.$ test, $\left.p=0.0002\right)$. In the patients with positive staining for EBV viral replication was localised to pulmonary epithelial cells using the specific epithelial cell marker (m613) and immunoperoxidase staining confirmed the staining to be within type II alveolar cells. This is the first report of in vivo EBV replication within epithelial cells of the lower respiratory tract in an immunocompetent host. This suggests that EBV may be an immune trigger or contribute to lung injury in CFA, offering a new avenue of treatment.

Th2 lymphocytes predominate in the pulmonary interstitium of patients with cryptogenic fibrosing alveolitis (CFA)

WaH Wallace, EA RAMAge, D LAMB, SEM Howie Department of Pathology, Edinburgh University Medical School, Teviot Place, Edinburgh EH8 $9 A G$ CFA is characterised by a persistent inflammatory process in the lung which is believed to be immunologically mediated. As such the CF4 helper/inducer lymphocyte subset is likely to play a key role in the disease process. CD4 positive lymphocytes can be divided into Th1 and Th2 subtypes on the basis of the profile of cytokines which they produce $(\mathcal{F}$ Immunol 1986;136:2348-57). Cells associated with delayed type hypersensitivity reactions (Th1) are characterised by the production of IL-10 and INF $\gamma$, while those associated with the development of allergic type antibody responses are characterised by the production of IL-4 and IL-5. We examined open lung biopsy specimens from 10 patients with CFA [median age (range) 70 (61-78)] using a non-isotopic in situ mRNA hybridisation technique (Exp Dermatol 1992;1:230-5) and immunohistochemistry in order to identify cells positive for the key cytokines INF $\gamma$, IL-4, and IL-5. In situ hybridisation revealed that the majority of infiltrating cells with 
lymphocyte morphology contained detectable mRNA for each of the 3 cytokines. In contrast the immunohistochemical data revealed that while most cells were positive for IL- 4 and IL- 5 only a very few were positive for INF $\gamma$. This difference was quantified using an image analysis system (HOME) [median \% lymphocytes positive with antibody (range): IL-4 78\% (53-85); IL-5 76\% (55-83); INF $\gamma$ 5.6\% $(2 \cdot 9-6 \cdot 6)]$. This discrepancy between detectable mRNA and protein production has been previously noted in other situations (Immunology Today 1990;11:458-64) and suggests that care must be taken in interpreting data on cytokine production when only mRNA is measured. CFA is associated with a predominantly Th2 pattern of CD4 activation.

\section{Pulmonary vascular endothelial permeability in systemic scler-} osis: relationship to alveolar epithelial permeability and disease extent

JB CAILES, AU WELLS, R LANEY, CM BLACK, DM HANSELL, R COKER, RM DUBOIS Royal Brompton Hospital, London In patients with systemic sclerosis (SSc) endothelial abnormalities have been found in areas of histologically normal lung, suggestng endothelial abnormalities may precede fibrosing alveolitis (FA). Increased alveolar epithelial permeability is also seen in SSc, and is associated with subsequent lung function decline. We aimed to assess the relationship between endothelial permeability and both extent of FA and epithelial permeability in patients with SSc. Twenty-two non-smoking patients with SSc ( 17 women) of mean (SD) age $50(10.8)$ years were randomly selected. All underwent estimation of endothelial and epithelial permeability during one week. Endothelial permeability was assessed by measuring the pulmonary transvascular flux of ${ }^{99}$ indium-labelled transferrin corrected for changes in pulmonary blood volume using ${ }^{99 \mathrm{~m}} \mathrm{Tc}$-labelled red blood cells. Epithelial permeability was measured by the clearance of nebulised ${ }^{99 \mathrm{~m}} \mathrm{Tc}-\mathrm{DTPA}$ from the lung. The lung was divided into three zones (upper, middle and lower) and endothelial and epithelial permeability measured in each. Nineteen of the patients underwent concurrent $C T$ scans $(3 / 10 \mathrm{~mm}$ sections). Visual estimates were made by two observers at four anatomical levels of the percentage of abnormal lung (to the nearest $5 \%$ ) and combined to give an overall percentage of abnormal lung. Pulmonary endothelial permeability was significantly related to epithelial permeability in the lower lung zones only $(r=0.58, \mathrm{p}=0.005)$, no relationship being present in the mid and upper zones. Radiological abnormality was not associated with endothelial permeability as there was no relationship between CT extent of abnormality and endothelial permeability in any lung zone, and upper zone endothelial permeability in nine patients with no evidence of upper zone disease on CT scan was no different from patients with evidence of disease. Endothelial permeability did not differ regionally throughout the lung whilst, in contrast, both the extent of CT abnormality and the epithelial permeability were greater in the lower zones than the upper. We conclude that in SSc changes in pulmonary endothelial and epithelial permeability are associated only in the lower zones of the lung where fibrosis is maximal. In the lower zones of the lung the epithelial and endothelial abnormality may predispose to the development of fibrosis. [Supported by a grant from the Raynauds and Scleroderma Association UK.]

\section{Rapid clearance of inhaled ${ }^{99}$ Tc-DTPA independently predicts lung functional decline in fibrosing alveolitis associated with systemic sclerosis}

JB CAILES, AU WELLS, CM BLACK, R UNDERWOOD, RM DUBOIS Royal Brompton Hospital, London In patients with fibrosing alveolitis (FA) increased clearance of inhaled technetium-labelled diethylene triamine pentaacetate $\left.{ }^{99 \mathrm{~m}} \mathrm{Tc}-\mathrm{DTPA}\right)$ is a risk factor for decline in lung function, and the predictive value of the test is increased if two measurements are made. It is not known if the ${ }^{99 \mathrm{~m}} \mathrm{Tc}$-DTPA scan is independently predictive when demographic, disease and lung function variables are taken into account. This study aimed to assess the value of two ${ }^{99 \mathrm{~m}} \mathrm{Tc}-$ DTPA scans for prediction of lung function decline, and to determine if the predictive value was independent of other variables. Fiftyfour non-smoking patients $(39 \mathrm{~F} / 15 \mathrm{M})$, with systemic sclerosis and fibrosing alveolitis (FASSc), mean (SD) age $48(11 \cdot 7)$ years had two ${ }^{99 \mathrm{~m}}$ Tc-DTPA scans performed a median (interquartile range) of 12 (9-16) months apart. A clearance half time of less than 40 minutes was regarded as abnormal (A) and greater than $\mathbf{4 0}$ minutes normal (N). Two groups were defined on the basis of the two scan results: group 1, NN ( $n=13)$ or $\mathrm{AN}(\mathrm{n}=7)$; and group 2, AA $(\mathrm{n}=34)$. Survival analysis (life table) was utilised with an event defined as a $15 \%$ or greater fall in either forced vital capacity (FVC) or gas transfer (TLCO) from the time of the second scan. Proportional hazards regression analysis was utilised to assess the independence of ${ }^{99 \mathrm{~m}} \mathrm{Tc}-$ DTPA clearance in prediction of decline in lung function. Only two of 20 patients in group 1 experienced a significant fall in lung function compared with 17 of 34 in group $2, p=0.001$ (log rank test). The risk of a significant fall in lung function in group 2 remained highly significant $(p=0.007)$ when controlling for the effects of age, sex, past smoking history, initial lung function (TLCO), type of systemic sclerosis (limited $v$ diffuse disease) and treatment. The estimated hazard for lung function decline for patients in group 2 was 10.9 times that in group 1. Abnormal clearance of ${ }^{99 \mathrm{~m}} \mathrm{Tc}-\mathrm{DTPA}$ on two occasions independently predicts a markedly increased risk of lung function decline in patients with FASSc. [Supported by a grant from the Raynauds and Scleroderma Association UK.]

\section{Cryptogenic fibrosing alveolitis with normal lung volumes}

mj Doherty, mg pearson, pma Calverley Aintree Chest Centre, Fazakerley Hospital, Liverpool Cryptogenic fibrosing alveolitis (CFA) typically presents with a restrictive pulmonary defect and a low TLCo. However, relative preservation of lung volume has been reported in CFA patients with concomitant emphysema (Turner Warwick. Respir Med 1990). How frequently this occurs and its relationship to symptoms and smoking intensity is not known. We have therefore retrospectively analysed all patients with CFA who attended our laboratory for full pulmonary function tests in the last three years. CFA was the physician diagnosis based on clinical examination, chest radiography, lung function tests and CT scans (28 cases) excluding cases associated with connective tissue disease and asbestosis. There were 41 patients of whom 20 had a \% predicted FVC of more than $80 \%$ (group A) and 21 less than $80 \%$ (group B). Group A patients

\begin{tabular}{|c|c|c|c|c|}
\hline & \multicolumn{2}{|l|}{ Group $A$} & \multicolumn{2}{|l|}{ Group B } \\
\hline & Actual (SE) & $\%$ Predicted & Actual (SE) & $\%$ Predicted \\
\hline $\begin{array}{l}\text { FVC } \\
\text { FEV } \\
\text { TLC } \\
\text { RV } \\
\text { TLCo } \\
\text { Kco }\end{array}$ & $\begin{array}{r}3.6(0.2) \\
2.6(0.1) \\
5.5(0.3) \\
1.9(0.2) \\
11.9(0.9) \\
2.5(0.2)\end{array}$ & $\begin{array}{l}93 \\
94 \cdot 7 \\
92 \cdot 4 \\
94 \\
48 \cdot 8 \\
58 \cdot 4\end{array}$ & $\begin{array}{c}2.1(0 \cdot 1) \\
1.8(0 \cdot 1) \\
3.5(0 \cdot 2) \\
1.34(0 \cdot 1) \\
11.9(0 \cdot 8) \\
3.8(0 \cdot 3)\end{array}$ & $\begin{array}{l}64 \cdot 4 \\
78 \cdot 6 \\
62 \cdot 6 \\
62 \\
49 \cdot 5 \\
85 \cdot 8\end{array}$ \\
\hline
\end{tabular}

were more likely $\left(\chi^{2}\right.$ test, $\left.\mathrm{p}<0.05\right)$ to be male $(75 \% v 38 \%)$, to have finger clubbing $(65 \% v 28 \%)$, and to present with non-respiratory symptoms $(25 \% v 0 \%)$. They were more likely to be smokers or exsmokers and had smoked more (40 $v 29$ pack years). Self reported exercise tolerance, age (61 $v 63$ years) and time to diagnosis were similar in each group, as was Trco; however, Kco was significantly lower in group A patients. Group A were more likely to have emphysema reported on CT scan (7 of $12 v 5$ of 16 scans) but the difference was not significant. In our laboratory CFA often presents without substantial volume restriction. Whether this reflects a different form of the disorder, a different stage of the disorder or an interaction with emphysema needs further elucidation.

Relationship between HIV-1 proviral DNA copy number in alveolar macrophages and clinical disease in the lung of AIDS patients

JR CLARKE, AJ GATES, RJ COKER, JD WILLIAMSON, DM MITCHELL Department of Medical Microbiology, St Mary's Hospital Medical School, London A study was performed to determine HIV-1 proviral DNA copy number in cells from the lung of AIDS patients and to investigate possible correlation with clinical disease. A total of 66 AIDS patients undergoing fibreoptic bronchoscopy for new respiratory episodes were studied. Alveolar macrophages (AM) were purified by adherence to plastic culture plates. Quantitative polymerase chain reaction (PCR) of HIV proviral DNA sequences was carried out using two different methods; an in house radiometric method and the Amersham Quant Amp scintillation proximity assay. 
Samples from 15 patients were also tested by limited cell dilution. The mean HIV-1 proviral DNA copy number was higher in the AM of patients where a specific opportunistic pathogen had been recovered from the lung ranging from 910 to $1169 \mathrm{HIV}$ copies $/ 10^{6} \mathrm{AM}$ compared to $281 \mathrm{HIV}$ copies $/ 10^{6} \mathrm{AM}$ in individuals where no respiratory pathogen was isolated. Median HIV copy number values in patients with PCP (200 copies $/ 10^{6} \mathrm{AM}$ ) or with bacterial pneumonia (600 copies/ $10^{6} \mathrm{AM}$ ) were higher than in individuals with no recoverable pathogen in the lung (10 copies $/ 10^{6} \mathrm{AM}$ ). Median HIV copy number in AM was found to be lowest in individuals undergoing their first respiratory episode (8 copies $/ 10^{6} \mathrm{AM}$ ) compared to patients with recurrent respiratory disease (200 copies $\left./ 10^{6} \mathrm{AM}\right)$. Sequential samples taken from 6 patients with repeated episodes of respiratory disease showed that there was an increased HIV DNA copy number in AM of three out of six (50\%) patients as disease progressed. HIV can be found in significant quantities in the AM of AIDS patients. HIV DNA copy number is highest as a consequence of opportunistic infection of the lung and is lowest when no pathogen can be recovered from the lung although this difference was not statistically significant $(p>0 \cdot 1)$. HIV proviral DNA copy in AM increases with disease progression.

\section{Quantity of HIV-1 proviral DNA in alveolar cells may be contributing to abnormalities of lung physiology in AIDS patients}

JR CLARKE, AJ GATES, RJ COKER, JD WILLIAMSON, JRW HARRIS, DM MITCHell Department of Medical Microbiology, St Mary's Hospital Medical School, London Measurement of transfer factor for lung carbon monoxide (TLCO) has been shown to be a sensitive method for the detection of respiratory physiological abnormalities in HIV seropositive individuals in the absence of overt lung disease. Sequential testing of lung function in asymptomatic HIV-1 seropositive patients showed that individuals with a TLCO value of $<80 \%$ predicted normal progressed significantly faster to AIDS than individuals $>80 \%$ TLCO value. Previously we have reported that mean TLCo values were lower at presentation in patients with more advanced HIV disease compared to asymptomatic individuals. In this study we have used quantitative PCR to investigate the relationship between HIV DNA copy number and TLCo. HIV-1 proviral DNA was detected by PCR in the bronchoalveolar lavage (BAL) cells of 82 out of $124(66 \%)$ HIV seropositive individuals undergoing diagnostic fibreoptic bronchoscopy for respiratory disease. Successful recovery of infectious HIV-1 from BAL cells by co-cultivation was achieved from 58 out of $100(58 \%)$ individuals indicating that, in the majority, HIV-1 is replicationcompetent. Detection by PCR or isolation of HIV from BAL cells alone was not related qualitatively to TLCo values in this patient group. However, quantification of HIV proviral DNA by PCR techniques revealed that both the mean and median HIV proviral DNA copy number increased from a mean (SD) of $10(13.5)$ and 7.5 (median) in individuals with a TLCo value of greater than 70 predicted normal to 625 (1088) and 100 DNA copies per $10^{6} \mathrm{BAL}$ cells in patients with a TLCo of between 40 and 49 predicted normal. The highest HIV load of 1472 (1680) and 1800 (median) DNA copies per $10^{6}$ BAL cells was observed in individuals with a TLCo of less than 29 predicted normal. These differences in the quantity of HIV proviral DNA were statistically significant $(p<0.01)$. We conclude that there is a relationship between HIV-1 load in the lung and abnormal pulmonary physiology of AIDS patients.

\section{Regional microbiology of the lung in patients with cystic fibrosis}

DL SMITH, EG SMITH, DE STABLEFORTH, ME KAUFMANN, TL PITT Adult Cystic Fibrosis Unit, Birmingham Heartlands Hospital; Central Public Health Laboratory, London Recent applications of genotyping methods to distinguish strains of Pseudomonas aeruginosa $(\mathrm{Pa})$ and Pseudomonas cepacia $(\mathrm{Pc})$ in the sputum of cystic fibrosis $(\mathrm{CF})$ patients have provided insight into the frequency of multiple strain carriage within individuals that may relate to the interpretation of epidemiological investigations. We have investigated multiple strain carriage by sampling directly from the post-mortem lung. Swabs were obtained from both lungs and the major airways of five CF patients at post-mortem. $\mathrm{Pa}$ isolates from these swabs were genotyped using the tox A gene probe and CHEF typing. Ninety percent of swabs produced successful cultures. Four patients grew $\mathrm{Pa}$ from all sites cultured, one patient grew $\mathrm{Pa}$ from two lobes and the major airways and $\mathrm{Pc}$ from all lobes and the major airways. In four patients, including the patient with co-existent $\mathrm{Pc}$ infection, all $\mathrm{Pa}$ isolates for each individual were indistiguishable by both genotyping methods. In addition $\mathrm{Pa}$ isolates from two of these patients were indistinguishable. In one patient three genotypically distinct strains of $\mathrm{Pa}$ were found in different anatomical sites; not all were reflected in major airway cultures. The finding of co-existent $\mathrm{Pa}$ and $\mathrm{Pc}$ infection in one patient is in keeping with our experience of Pc infection in $\mathrm{CF}$ patients. One of five patients studied was found to exhibit multiple strain carriage which was only partially reflected by major airway culture. The finding of genotypically indistinguishable strains in two patients supports the possibility of patient to patient transmission as a source of infection. In general, our study supports the notion that major airway sputum accurately reflects the microbiological flora present at more peripheral sites. However, we have shown that in cases exhibiting multiple strain carriage not all strains present in the periphery may be reflected in major airway cultures.

$\beta_{2}$-integrin expression on circulating blood neutrophils from patients with cystic fibrosis

D O'RIORDAN, J HAYES, C O'CONNOR, MX FITZGERALD Department of $\vec{x}$ Medicine, University College Dublin and St Vincent's Hospital, of Dublin Lung disease is the major cause of morbidity and mortality in cystic fibrosis (CF). Lung destruction results from chronic pulmonary it inflammation, characterised by the continual influx of neutrophils from the bloodstream. Recently, hyperexpression of the $\beta_{2}$-integrin (CD18) adhesion molecules on circulating neutrophils has been $\frac{\mathrm{O}}{3}$ observed in patients with ARDS (Laurent et al. Am $\mathcal{F}$ Respir Crit Care Med 1994;149:1534-8). It is suggested that this may contribute to $D$ the excessive pulmonary accumulation of neutrophils observed in ARDS. To assess whether similar mechanisms might be operating in $\mathrm{CF}$, we compared surface expression of CD18 on circulating neutrophils from $10 \mathrm{CF}$ patients $(7 \mathrm{M}, 3 \mathrm{~F}$; mean age $=20.6$, range of $21-28$ yrs) and 10 control subjects $(6 \mathrm{M}, 4 \mathrm{~F}$; mean age $=27 \cdot 7$, range 23-35). All CF patients displayed chronic pulmonary bacterial colonisation but none were acutely infected at the time of sampling. As assessed by pulmonary function measurements, lung disease severity ranged from mild $\left(\% \mathrm{FEV}_{1}=85 ; \% \mathrm{FVC}=102\right)$ to severe $\left(\% \mathrm{FEV}_{1}=\right.$ $27 ; \% F V C=44$ ) within the CF study group. Peripheral blood leukocytes were labelled with an FITC-conjugated monoclonal antibody to CD18 and analysed by flow cytometry. Results were calculated as the mean fluorescence intensity (MFI) of CD18-labelled neutrophils. No difference in surface $\beta_{2}$-integrin expression on circulating neutrophils between CF patients $(\mathrm{MFI}=54.6(15 \cdot 8))$ and control subjects $(\mathrm{MFI}=57.2(10.8))$ was observed. Only one CF patient displayed an MFI value above normal range (i.e. $>$ mean control value $+2 S D$ ). Thus, in CF over-expression of $\beta_{2}$-integrins on circulating neutrophils does not seem to contribute to the excessive accumulation of neutrophils in the lung. [This work was supported by the Cystic Fibrosis Association of Ireland.]

Effect of recombinant human DNase in vitro and in vivo in
bronchiectasis
bronchiectasis

RA STOCKLEY, VC OKAFOR, C LLEWELLYN-JONES, SL HILL Lung Im- N munobiochemical Research Laboratory, The General Hospital, Steelhouse Lane, Birmingham Neutrophil recruitment and the release of elastase into the bronchial secretions is thought to play a significant role in the pathogenesis of chronic bronchial diseases such as bronchiectasis with and without cystic fibrosis. Preliminary clinical studies have of investigated the role of inhaled human recombinant DNase in the management of these conditions. However, mucus may entrap several factors that play a part in the pathogenesis of this disease including chemotactic factors and neutrophil elastase itself. Thus disruption of the mucus by DNase may release these factors and adversely affect $\mathbb{D}$ the lung. We have studied the effect of DNase in vitro and in vivo $\sigma$ upon sputum sol phase chemotactic and elastase activity. DNase treatment of five sputum samples in vitro did not affect the amount of albumin recovered or the chemotactic activity; however elastase activity rose from a mean (SE) of $16.2(3.6)$ elastase units to 46.3 $(8 \cdot 7)(p<0.05)$. Twenty bronchiectatic patients entered a double blind $\supset$ study of inhaled DNase $(2.5 \mathrm{mg}$ twice a day). Ten received placebo and 10 active treatment. During the run in period the two groups were similar with respect to the degree of lung inflammation as defined by albumin leakage into the lung (mean placebo sputum/serum ratio = $3.35(1.52) \times 10^{-2}$; treatment group $\left.=2.35(0.59)\right)$, chemotactic activity (placebo mean $=29.4(5.4)$ cells/field; treatment $=32.3(2.84)$ ) and elastase activity (placebo mean $=33 \cdot 7(7 \cdot 4)$; treatment $=75 \cdot 7$ 
(24.3)). No change in any of these parameters was seen over the first month in either the placebo or treatment group. In particular the sputum elastase activity did not increase significantly on therapy (day $1=38.3(12.4)$; day $8=57 \cdot 7(20.3)$; day $29=57.9(18.3))$. Thus, despite the in vitro changes inhalation of DNase does not appear to adversely affect factors that may influence lung damage in bronchiectasis.

\section{Liposome-mediated CFTR gene transfer to the nasal epi-} thelium of patients with cystic fibrosis

EWFW ALTON, N CAPLEN, PG MIDDLETON, JR DORIN, BJ STEVENSON, $X$ GAO, SR DURHAM, PK JEFFERY, ME HODSON, C COUTELLE, L HUANG, DJ PORTEOUS, R WILLIAMSON, DM GEDDES Ion Transport Unit, National Heart and Lung Institute, London; Department of Biochemistry, St Mary's Hospital Medical School, London; MRC Human Genetics Unit, Edinburgh; Department of Pharmacology, University of Pittsburgh, USA; Departments of Allergy and Clinical Immunology, Lung Pathology and Cystic Fibrosis, National Heart and Lung Institute, London We have carried out a phase I double blind placebo controlled clinical trial of cationic liposome-mediated CFTR gene transfer in 15 cystic fibrosis (CF) patients. Subjects ( $n=3$ each group) received either $10 \mu \mathrm{g}$, $100 \mu \mathrm{g}$, or $300 \mu \mathrm{g}$ of $C F T R \mathrm{cDNA}$ or the appropriate liposome only dose $(n=6)$, applied topically to each nostril. There were no adverse clinical effects and nasal biopsies demonstrated no histological differences between the groups. Plasmid derived CFTR mRNA was detected in six of the eight available samples from the CFTR treated subjects. In vivo assessment of nasal electrophysiological parameters demonstrated an approximately $20 \%$ restoration of the deficit $(\mathrm{p}<0.05)$ in both chloride and sodium abnormalities between non-CF and $\mathrm{CF}$ subjects. These changes were maximal around day 3 following administration and had reverted to pretreatment values by day 7 . In some cases the chloride responses of the CFTR treated subjects reached the range for non-CF subjects. These data are encouraging but suggest that transfection efficiency may need to be improved.

\section{A progressive care programme for patients failing to wean on} an ICU: clinical experience

JM SHNEerson, IE SMITH Respiratory Support Centre, Papworth Hospital, Cambridge During the 32 month period from January 199240 patients (21 women), mean (SD) age $54(16.5)$ years entered our progressive care programme (PCP) because of a failure to be weaned from mechanical ventilation. The primary diagnosis was pulmonary in 14 patients (COPD 10, post TB sequelae 4), chest wall deformity in five and neuromuscular disease in 21 . The mean length of stay on the ICU at the referring hospital was 32 days. Twelve patients had failed at least one trial of extubation, 29 patients had been tracheostomised, and 30 patients were completely ventilator dependent. The mean duration of assisted ventilation at the time of transfer was 19.4 hours per day, with 28 patients requiring additional oxygen (mean $\mathrm{FiO}_{2} \mathrm{0} \cdot 38$ ). The mean (SD) $\mathrm{PCO}_{2}$ immediately prior to transfer was $6.95(1.61) \mathrm{kPa}, 11$ patients having a $\mathrm{PCO}_{2}>7.0 \mathrm{kPa}$. On transfer to the PCP the diagnosis was reviewed in each case and in 10 patients additional diagnostic information resulted in changes of management - for example, withdrawal of high dose steroids in three patients. In all patients ventilatory, nutritional medical, psychological and communication requirements were reassessed and non-invasive methods of monitoring and ventilation were introduced where possible. The mean length of stay on the PCP was $26.9(16 \cdot 2)$ days. The hospital survival was $95 \%$. Of the survivors 32 patients $(84 \%)$ were discharged directly to home, three $(8 \%)$ to residential care, and three $(8 \%)$ returned to the referring hosital. Twelve patients required no ventilatory support at discharge although five retained a tracheostomy. Nineteen patients were discharged with nasal intermittent positive pressure ventilation (NIPPV) and five with negative pressure cuirass ventilation. Of this group of 24 patients five used the equipment for more than eight hours each day. Two other patients remained on IPPV via tracheostomy (one for 16 hours a day and one for 22 hours each day). Only two patients required additional oxygen at home. Mean (SD) overnight $\mathrm{SaO}_{2}$ at discharge was $91(23.74) \%$ with daytime $\mathrm{Po}_{2}$ of $10.3(3.36) \mathrm{kPa}$ and $\mathrm{PCO}_{2}$ of $6.16(0.78) \mathrm{kPa}$ breathing air. These findings indicate that most patients who fail to be weaned from mechanical ventilation with conventional techniques can do so and return to their homes with the aid of a comprehensive PCP incorporating the facility of non-invasive home ventilatory support.
Supplementary oxygen administration with nasal intermitent positive pressure ventilation

AJ PADKIN, WJM KINNEAR Queen's Medical Centre, University Hospital, Nottingham Intermittent positive pressure ventilation via a nasal mask (nIPPV) can be used to prevent hypercapnia when oxygen is administered to patients who are dependent on hypoxic respiratory drive. We have investigated the inspired oxygen concentration $\left(\mathrm{FIO}_{2}\right)$ achieved when oxygen is added at different points in the breathing circuit. We studied 11 patients (four women) with chest wall disease, all of whom were accustomed to nIPPV. Oxygen was administered at rates of $1-6 \mathrm{l} / \mathrm{min}$ by three different routes: route $\mathrm{A}$, directly into the ventilator (Monnal) with $\mathrm{FiO}_{2}$ remaining constant at a known value throughout inspiration; route $\mathrm{B}$, into the tubing connecting the ventilator to the patient close to the ventilator outlet port; route $\mathrm{C}$, into the nasal mask. By comparing each patient's $\mathrm{SaO}_{2}$ (Ohmeda 3700) with routes $B$ and $C$ to that obtained with route $A$ we estimated the $\mathrm{FIO}_{2}$ for different oxygen flow rates. The mean $\mathrm{FiO}_{2}$ for route $\mathrm{C}$ is

\begin{tabular}{lrrrrrr}
\hline $\mathrm{O}_{2}(1 / \min )$ & 1 & 2 & 3 & 4 & 5 & 6 \\
$\mathrm{FIO}_{2}(\%)$ & 31 & 37 & 42 & 44 & 45 & 47 \\
\hline
\end{tabular}

shown in the table. There was no significant difference in $\mathrm{FiO}_{2}$ between routes $B$ and $C$ at any flow rate. Route $B$ is preferable to route $C$ in ventilators without the facility for route $A$ since it achieves a similar $\mathrm{FiO}_{2}$ but does not require additional tubing attached to the mask, and is less liable to become disconnected when the patient moves.

Comparison of "elective" and "emergency" initiation of nocturnal assisted ventilation: importance of avoiding endotracheal intubation

PH JOHNSON, BG COOPER, L WATSON, JR BRITTON, WJM KINNEAR Nottingham Assisted Ventilation Group, City and University Hospitals, Nottingham Most patients referred for consideration of nocturnal assisted ventilation (AV) report a gradual decline in health, often over several years. Some of these patients nevertheless present with life threatening acute respiratory failure and require emergency endotracheal intubation. We have compared this group with those presenting earlier, in whom AV can be commenced "electively". Since 198820 patients (eight women) have been commenced on nocturnal AV, 19 with IPPV (18 via a nasal mask and two through a tracheostomy) and one with external negative pressure ventilation. Seven patients required intubation and ventilation on the intensive care unit (ICU) at first presentation. These seven patients spent a mean (SD) of $21.9(13.0)$ days on ICU and a total of $42.9(11.3)$ days in hospital. The other 13 patients spent $0.8(1.5)$ days on ICU and $11.0(10.8)$ days in hospital $(p<0.01)$. There were no significant differences between the two groups in age, vital capacity, or risk factors for the development of respiratory failure, and the aetiology of respiratory failure was similar. The importance of early referral of patients at risk of nocturnal hypoventilation to an appropriate centre needs to be emphasised to GPs and hospital physicians. At presentation, every effort should be made to use non-invasive $\mathrm{AV}$ techniques and avoid endotracheal intubation.

Outcome of nasal intermittent positive pressure ventilation in acute respiratory failure

JS BROWN, DJ MEECHAM JONES, JA WEDZicha Department of Thoracic Medicine, The London Chest Hospital, London Nasal intermittent positive pressure ventilation (NIPPV) has been used in the treatment of acute-on-chronic respiratory failure, although the acceptability and success rate of the technique is variable and has not been evaluated. We report a survey of 101 consecutive episodes of acute respiratory failure treated with NIPPV in 50 patients $(25 \mathrm{M}, 25 \mathrm{~F}$, median age 69 years, range $45-78$ ) over an 18 month period. Thirty-three patients $(66 \%)$ had COPD, $11(22 \%)$ restrictive chest wall disease, and six $(12 \%)$ obstructive sleep apnoea. Thirty-two $(64 \%)$ patients were using domiciliary LTOT and nine $(18 \%)$ were on home NIPPV. Twenty-eight $(56 \%)$ patients had a single admission, $22(44 \%)$ patients had multiple admissions (median 3, range 2-10). Data from the first admission only were included in the analysis. All patients were managed on general wards. Admission (pretreatment) blood 
gases $(51 \%$ of patients on supplementary oxygen) were (mean (SD)): pH 7.34 (0.08); $\mathrm{PaCO}_{2}$ 9.21 (2.0); $\mathrm{PaO}_{2} 7.04$ (2.5). All patients received standard therapy, including supplementary oxygen. There were $15(30 \%)$ treatment "failures". Eleven $(22 \%)$ patients complied poorly with NIPPV, of whom two died; two patients were intubated and ventilated whilst using NIPPV, one of whom died; and two compliant patients died during admission. There were proportionally fewer failures in patients with restrictive chest wall disease compared with COPD (1/11 patients $v 12 / 32)$, but the difference was not statistically significant. Admission $\mathrm{pH}, \mathrm{PaCO}_{2}$ and age did not predict failure. Mean (SD) blood gases on NIPPV shortly after admission showed a significant improvement from admission: pH 7.37 (0.07), $\mathrm{PaCO}_{2} 8.36$ (1.68), $\mathrm{PaO}_{2}$ 9.53 (2.85); mean improvements (95\% CI) were $\mathrm{pH}+0.035(0.006$ to $0.63, \mathrm{p}=0.018), \mathrm{PaCO}_{2}-0.88(-0.27$ to $-1.50, \mathrm{p}=0.006), \mathrm{PaO}_{2}+2.51(1.21$ to $3.81, \mathrm{p}<0.001)$. Patients with an admission $\mathrm{PaO}_{2}$ of $6.6 \mathrm{kPa}$ or lower have been identified as at high risk (Jeffrey, Thorax 1992;47:34-40). NIPPV raised $\mathrm{PaO}_{2}$ from an admission level of $6.6 \mathrm{kPa}$ or less in 16 out of 19 patients $(\mathrm{p}=$ 0.006 ). Median duration of NIPPV use was eight days (range 1-47) and of hospital stay 17 days (1-63). NIPPV produced rapid improvement in blood gases in patients treated on general wards for both obstructive and restrictive lung disease. The technique was used successfully in $70 \%$ of unselected patients with an acute deterioration of their chronic respiratory failure.

Use of the Hayek oscillator in stable hypercapnic obstructive airways disease

FM HARDINGE, JR STRADLING Osler Chest Unit, Churchill Hospital, Oxford The Hayek oscillator is a new technique which consists of a negative pressure cuirass capable of operating at a variety of high frequencies. It could potentially be used to provide assisted ventilation to patients presenting with respiratory failure resulting from an acute exacerbation of chronic obstructive airways disease. We studied the effect of short term periods of ventilation in 12 subjects with stable hypercapnic chronic airways disease who were outpatients. Subjects received, in a random order, five minute periods of ventilation at three different frequencies, with a "sham" or control period of ventilation at each frequency. Measurements were made before and after ventilation, of changes in gas exchange (oxygen saturation, end tidal $\mathrm{CO}_{2}$ ), spontaneous respiratory rate (oronasal airflow, inductance plethysmography) and blood pressure (Finapres). In addition, arterial blood gases were measured in four subjects. Each outcome measure was analysed separately for the factors affecting it (ANOVA, Duncan's post hoc analysis). There was no significant improvement in oxygen saturation, end tidal $\mathrm{CO}_{2}$ or arterial blood gas measurements during any of the "sham" or "active" periods of ventilation. There was significant intersubject variation in respiratory rate $(p=0.0001)$, but no significant changes with ventilation at any setting. No periods of apnoea were seen in any subjects. Blood pressure was also not affected by use of the cuirass. We conclude that the Hayek oscillator does not ventilate patients with stable chronic obstructive airways disease when used for short periods at frequencies of 30-90 oscillations/minute. This is in contrast to experience with normal subjects, and may be due to the decreased chest compliance, altered respiratory drive, and increased anxiety in this group of patients.

\section{Magnetic resonance imaging (MRI) in the staging of bronchial} carcinoma

AH AL-GHAMDI, CA ROOBOTTOM, M WESTON, C FORRESTER-WOOD, $\mathrm{K}$ JEYSINGHAM, W DIHMIS, P GODDARD, JR CATTERALL Respiratory and Radiology Departments, Bristol Royal Infirmary; Thoracic Surgery Unit and MRI Centre, Frenchay Hospital, Bristol The role of MRI in the staging of bronchial carcinoma is undefined. Fifty patients considered to have operable bronchial carcinoma on conventional staging including computed tomography (CT) underwent subsequent MRI scanning in the week prior to operation. Axial STIR and T1 weighted images before and after intravenous gadlinium-DPTA and multiecho proton density and T2 weighted coronal images were performed. Assessment of local spread to pleura, chest wall and mediastinum as well as nodal status was made at surgery and by subsequent histopathological examination of resected tissue, and the results compared with the MRI findings. Surgery was performed without the knowledge of the MRI findings. Mediastinal nodes of $0.75 \mathrm{~cm}$ or more were demonstrated by MRI with a sensitivity of $92 \%$, specificity of $100 \%$, and accuracy of $96 \%$. Regarding hilar lymphadenopathy the results were $76 \%, 100 \%$ and $85 \%$, respectively. However, lymph node enlargement correlated poorly with the presence of malignancy in the nodes. For pleural involvement the results were $81 \%, 90 \%$ and $87 \%$, respectively. These results are comparable to published results for CT. Regarding mediastinal invasion MRI had a sensitivity of $93 \%$, specificity of $94 \%$, and accuracy of $93 \%$. These results are superior to those reported for CT. In conclusion, MRI is as sensitive as CT in the demonstration of mediastinal and hilar lymph node enlargement, chest wall, and pleural invasion. It is, however, superior in the demonstration of mediastinal invasion and thus holds promise for more accurate staging of bronchial carcinoma preoperatively.

Age as a factor in lung cancer surgery results from one region over a five year period

GE WILSON, CIA JACK, M LYE, MJ DRAKELEY, RJ DONNELLY, CRK HIND The Cardiothoracic Centre, Liverpool; Royal Liverpool University Hospital, Liverpool Lung cancer is now the commonest cause of cancer deaths in men in Great Britain and accounts for $8 \%$ of all male and $4 \%$ of all female deaths. The prognosis for non-small cell cancer remains poor and surgery remains the best chance of cure. Despite this, only $15 \%$ of patients have attempted curative surgery. For a similar stage of lung cancer, patients aged 65 or over are less likely to be operated on. We have therefore looked at the situation over a five year period in Mersey region. A total of 912 thoracotomies (464 lobectomies, 313 pneumonectomies, and 135 wedge resections) were performed from August 1987 to July 1992. We examined the records of all patients aged 70 or above undergoing surgery for lung cancer (elderly). A random group of patients below this age was selected for comparison (young). Seventy nine elderly patients (50 men; mean age 75 , range $70-87$ ) and 83 young patients (53 men; mean age 60, range 34-69) underwent thoracotomy for bronchial carcinoma. The characteristics for the two groups are as shown in the table. The type of operation performed, tumour cell type and

\begin{tabular}{llll}
\hline & Elderly & Young & $p$ \\
\hline FEV $_{1}$ (\%pred) & $67 \cdot 6(2 \cdot 1)$ & $70 \cdot 4(2 \cdot 4)$ & NS \\
FVC (\%pred) & $84 \cdot 3(2 \cdot 3)$ & $85 \cdot 7(2 \cdot 4)$ & NS \\
Smoker (ever) & $91 \%$ & $99 \%$ & NS \\
Smoker (current) & $38 \%$ & $63 \%$ & NS \\
Prev med history & $84 \%$ & $67 \%$ & $0 \cdot 02$ \\
Medication & $59 \%$ & $53 \%$ & NS \\
Time to op (days) & $84(13)$ & $36(4)$ & 0.001 \\
Abnormal ECG & $38 \%$ & $20 \%$ & 0.02 \\
\hline
\end{tabular}

stage were similar for each group. Postoperative complications were fewer in the elderly (26\%) than in the young $(38 \%)$, due mainly to chest infections. Adjuvant radiotherapy was used in $26 \%$ of elderly and $18 \%$ of young patients. The mean survival for each group was 23 (2) months. From these figures it would appear that age should not necessarily be considered a contraindication to surgery for lung cancer. Furthermore, patients over the age of 70 have fewer postoperative complications and equal survival figures to younger patients, despite having to wait longer for referral.

\section{Does blood transfusion affect long term survival following lung} cancer resection?

RK MACKENZIE, MM KENNEDY, M GREISS, KM KERR, RR JEFFREY Departments of Cardiothoracic Surgery, Transfusion Medicine and Pathology, Aberdeen Royal Infirmary NHS Trust, Aberdeen Debate continues on the potential deleterious effects of perioperative blood transfusion in patients undergoing potentially curative resections for cancer. We have studied the results of 280 patients who underwent pulmonary resection for non-small cell lung cancer between January 1986 and January 1991 looking at the effect of perioperative blood transfusion on long term survival. Demographic data were obtained from the case records, perioperative transfusion data were obtained from the Regional Blood Transfusion Service records, and the pathology was reviewed. Date of death was obtained from the Scottish Cancer Registry. Patients dying within 90 days of operation $(n=26)$ were excluded. The mean age of the group was $62.3(8 \cdot 8)$ years and $65.7 \%$ were men. There were 116 squamous carcinomas, 89 
adenocarcinoma, 21 of mixed differentiation, 11 large cell carcinomas, and 17 others. Eighty five patients underwent pneumonectomy, 148 lobectomy or bilobectomy, and 21 other procedures, e.g. segmentectomy. Three year survival for pneumonectomy was $44.7 \%$ compared with $60.5 \%$ for lobectomy; $125(49 \cdot 2 \%)$ patients had stage I disease, $79(31 \cdot 1 \%)$ stage II and $50(19 \cdot 7 \%)$ had stage III. Patients with stage I disease survived a mean of $46.4(24.2)$ months with a three year survival of $64.8 \%$, those with stage II 40.0 $(25 \cdot 8)$ months and $53 \cdot 8 \%$, and for stage III $31 \cdot 1(26 \cdot 4)$ months and $40 \%$. Over all stages patients with adenocarcinoma did marginally worse than those with squamous tumours (e.g. stage I $67 \cdot 3 \% v$ $63 \cdot 8 \%$ ). For all stages of disease patients transfused perioperatively had significantly reduced long term survival e.g. stage I not transfused $50.8(23.6)$ months $v$ transfused $40.2(26.4)$ months, $\mathrm{p}<0.01$. We conclude that perioperative blood transfusion should be considered among the major determinants of long term survival following resection for lung cancer.

Patient survival and bronchial resection line status in primary lung carcinoma

KK TAN, MM KENNEDY, KM KERR, RR JEFFREY Departments of Cardiothoracic Surgery and Pathology, Aberdeen Royal Infirmary A series of 255 patients who had lung resection for primary non-small cell cancer between 1986 and early 1991 were studied. Postoperative survival was calculated from Cancer Registry data; patients surviving <3 months were excluded. All histological material was reviewed. From 255 cases $18(7 \%)$ showed tumour at the bronchial resection line (BRL), six (2.4\%) cases showed carcinoma in situ (CIS), and five $(2 \%)$ showed dysplasia. Of the 18 cases of BRL with tumour five showed mucosal disease only while 13 showed extrachondral tumour. Mean survival for those with invasive disease at the BRL was 32.6 (24.7) months compared with $41.7(26.0)$ months for those without $(p=0.0004)$. There was no significant difference in mean survival between the groups with CIS, dysplasia, or benign BRL, respectively. The case group with only mucosal tumour at the BRL, but also the group with extrachondral tumour at this site, had $40 \%$ of patients alive at the review date ( $\geqslant 46$ months postoperatively). Of those who died before the review date mean postoperative survival with only mucosal disease at BRL was $29 \cdot 1$ months, while it was only 11 months for the group with BRL showing extrachondral cancer. These data suggest that invasive disease, and particularly extrachondral malignancy, but not carcinoma in situ or dysplasia may be a relevant factor in determining patient survival after lung resection for primary bronchial carcinoma.

TNM staging system for malignant mesothelioma: evaluation in 88 patients with malignant pleural mesothelioma

L TAMMILEHTO, $\mathrm{K}$ MATTSON, L KIVISAARI, U-S SALMINEN, $\mathbf{P}$ MAASILTA Department of Epidemiology and Biostatistics, Institute of Occupational Health, Helsinki; Departments of Pulmonary Medicine, Diagnostic Radiology, and Thoracic and Cardiovascular Surgery, Helsinki University Central Hospital, Helsinki, Finland There is no universally recognised method for staging malignant mesothelioma, although computed tomographic (CT) scanning has improved non-invasive staging. IUAC has recently proposed using the TNM staging system for mesothelioma, but in clinical practice it is difficult to assess the tumour (T) and nodal $(\mathrm{N})$ involvement due to the unique plate-like growth pattern of this tumour. In order to evaluate TNM staging we analysed preoperative CT scans from 88 patients with histologically confirmed malignant pleural mesothelioma. Most of the patients would participate in a clinical study programme which included debulking surgery, chemotherapy and hemithorax irradiation. Median age of patients was 56 years (range 38-79). There were 70 men and 18 women, and 33 had tumours with epithelial histology. Median survival for all the patients, measured from the data of histological confirmation of mesothelioma, was 10 months (range $0 \cdot 2-110$ ). The same radiologist (LK) analysed CT scans according to the TNM staging system. Actuarial survival curves were constructed by the Kaplan-Meier method. Survival curves for the different TNM categories were compared using the log rank test. Node evaluation could not be completed in eight cases because the tumour had encompassed the hilum and mediastinum. In multivariate analysis significant differences in prognosis correlated to the different $T$ categories $(p<0.01)$, and the different stage categories $(\mathrm{p}<0.05)$, but not to the $\mathrm{N}$ or $\mathrm{M}$ categories. Larger studies are needed to assess the importance of TNM staging for selecting treatment and as a prognostic factor for malignant mesothelioma.

\section{Eight year experience with the pleuroperitoneal shunt for the} management of malignant pleural effusions

M PETROU, D KAPLAN, P Goldstraw Royal Brompton Hospital, London Malignant pleural effusions are a common cause of morbidity and patients often require repeated palliation by needle thoracocentesis, tube thoracostomy, and pleurodesis. For patients where lung expansion is restricted by a fibrous or malignant cortex ("trapped lung") pleurodesis usually fails and an alternative method of treatment is needed. Over an eight year period (1986-94) 275 patients were referred to our surgical unit with pleural malignancies, 65 of whom had no significant effusion (underwent pleural biopsy only) or were discovered to have an empyema (requiring rib resection and external drainage). This left 210 patients with large recurrent effusions and 128 were treated by talc pleurodesis. We review the results of the remaining 82 patients who were treated with a pleuroperitoneal (Denver Biomedical Inc) shunt for recurrent malignant effusions associated with a trapped lung. The mean age was 60 years (range $25-85$ years) and 62 patients $(76 \%)$ had received repeated first line treatment before referral. The most common pathological diagnoses were mesothelioma (39 patients), secondary breast carcinoma (15 patients), and primary or secondary adenocarcinoma of the lung (12). All patients underwent general anaesthesia and thoracoscopy or minithoracotomy. The degree of lung expansion was assessed by sustained positive pressure ventilation and was found to be severely limited in all patients resulting in the insertion of a pleuroperitoneal shunt. There were no intraoperative deaths and only two early deaths $(2 \cdot 4 \%)$. The mean hospital stay was 5.9 days (range 2-12 days). Follow up data were complete in $85 \%$ of the patients. There were nine case (11\%) of blocked shunts (five requiring replacement or renovation and four requiring removal for sepsis) at one week to four months after insertion. One patient required further thoracocentesis and two patients developed malignant ascites. The median survival in this heterogeneous group of cancer patients was 5.4 months (range 1-56) and those with secondary breast carcinoma or lymphoma surviving the longest. Our results show that patients in whom pleurodesis would fail due to limited lung expansion despite adequate drainage of the effusion can obtain safe and effective palliation by pleuroperitoneal shunting.

Tetracycline pleurodesis in malignant pleural effusion: a comparison of needle aspiration with intercostal tube drainage

LG MCALPINE, JW KAY, NC THOMSON, BHR STACK, PA CORRIS, E NEVILLE, FJC MILLARD ON BEHALF OF THE BRITISH THORACIC SOCIETY RESEARCH COMmitten The British Thoracic Society, 1 St Andrews Place, London Chemical pleurodesis is often required to prevent reaccumulation of malignant pleural effusion. Tetracycline is the commonest agent used but the method of removing the pleural fluid varies (McAlpine et al, Thorax 1990;45:699-701). The present study compared the success of tetracycline pleurodesis using a needle aspiration technique with an intercostal tube drainage method in patients with recurrent malignant effusion. Patients with recurrent pleural effusion due to histologically or cytologically proven malignancy other than mesothelioma or lymphoma who were suitable for pleurodesis (lung fully expandable) were eligible for this multicentre trial. They were randomised to either needle aspiration of the effusion to dryness followed by instillation of $1.5 \mathrm{~g}$ tetracycline in $50 \mathrm{ml}$ saline containing $200 \mathrm{mg}$ lignocaine or to intercostal tube drainage to dryness prior to instillation of the same mixture and drainage then continued for $\geqslant 48$ hours or until $<100 \mathrm{ml} /$ day. A total of 113 patients were randomised but 32 were lost to follow up or were withdrawn. Thus there were 81 evaluable patients (lung cancer 34; breast 15; other/ unknown primary 32). Forty one were allocated needle aspiration and 40 an intercostal tube drain. Pleurodesis was successful (no need for further aspiration) at six weeks in 11 of $35(29 \%)$ in the needle aspiration group and in 29 of $36(80 \%)$ in the intercostal tube drain group ( $\mathrm{p}<0.0001)$. At 12 weeks the corresponding success rates were seven of $33(21 \%)$ and 25 of $32(78 \%)(p<0.0001)$, and at 24 weeks four of $30(13 \%)$ and 19 of $27(70 \%)(p<0.0001)$. There were five failures in each treatment group due to problems with the technique. The underlying diagnosis did not influence success. There were no differences in the time between pleurodesis and hospital discharge, 
in the complication rate, or in the acceptability to patients between the two techniques. In conclusion, intercostal tube drainage of pleural fluid when carrying out tetracycline pleurodesis for malignant effusion is considerably more effective than a simple needle aspiration method without being any less acceptable to patients.

\section{Mast cells infiltrate the bronchial epithelium in mild asthma}

M SYNEK, AJ FREW, FC LAMPE, P BRADDING, ST HOLGATE University Medicine, University of Southampton, Southampton We compared numbers and distribution of mast cells infiltrating the airways in eight mild asthmatic subjects who died of causes unrelated to asthma with nine non-asthmatic subjects who died of incidental causes. Specimens of lung tissue were obtained at post-mortem examination. Sections $4 \mu \mathrm{m}$ thick were cut from the tissue blocks and stained by monoclonal antibody (AA1:tryptase Moab) using streptavidin-biotin peroxidase technique. The numbers of positive cells were expressed in cells $/ \mathrm{mm}$ for the epithelium and cells $/ \mathrm{mm}^{2}$ for the submucosa. The airways were divided into two groups according to the internal perimeter ( $\mathrm{Pi}$ ): those with $\mathrm{Pi}>2 \mathrm{~mm}$ and those with $\mathrm{Pi}<2 \mathrm{~mm}$. All airways together were studied first, then larger and smaller airways were evaluated separately. When all airways were examined together we found higher numbers of mast cells infiltrating the epithelium in mild asthma than in non-asthmatic subjects $(2.06 v 0.17$ cells $/ \mathrm{mm}, \mathrm{p}=0.006)$. In the submucosa the numbers of mast cells were not significantly different $\left(103.4 v 112.7\right.$ cells $\left./ \mathrm{mm}^{2}, \mathrm{p}=0.597\right)$. The same applied to larger and smaller airways examined separately. The respective figures for epithelium were $1.31 v 0.23$ cells $/ \mathrm{mm}, \mathrm{p}=0.03$, and $1.99 v 0$ cells/ $\mathrm{mm}, \mathrm{p}=0.02$. The corresponding figures for the submucosa were $103.2 v 106 \cdot 1$ cells $/ \mathrm{mm}^{2}, \mathrm{p}=0.80$ and $130.6 v 155.0$ cells $/ \mathrm{mm}^{2}, \mathrm{p}=$ $0 \cdot 42$. These results indicate that mast cells infiltrate the bronchial epithelium in mild asthma, while the numbers of cells in the submucosa do not differ from normal subjects. This distribution of mast cells is present throughout the bronchial tree, irrespective of airway size. [This study was supported by Action Research.]

Allergen-induced late asthmatic reactions are associated with increased nitric oxide concentration in exhaled air

SA KHARITONOV, DJ EVANS, BJ O'CONNOR, PJ BARNES Department of Thoracic Medicine, National Heart and Lung Institute, London; Clinical Studies Unit, Royal Brompton Hospital, London The concentration of nitric oxide (NO) is increased in the exhaled air of asthmatic patients and may reflect cytokine-mediated inflammation in the airways. We have used inhaled allergen challenge (AC) to induce a late asthmatic reaction (LAR) to explore whether allergen-induced inflammation causes an elevation in the level of NO. We measured serial FEV 1 by spirometry and NO using a chemiluminescence analyser for up to 27 hours following allergen or methacholine challenge and measured responsiveness to inhaled histamine by determining the provocative concentration causing a $20 \%$ fall in $\mathrm{FEV}_{1}\left(\mathrm{PC}_{20}\right)$ before and three hours after challenge. Of 22 patients who underwent AC, 16 developed dual early and late responses, whereas six had a single early response. In the patients with a dual response the mean maximal fall in $F_{E V}$ during the LAR was $34.9(8.6) \%$ at 21 hours after challenge and there was a significant reduction in histamine PC $_{20}$ at three hours. In these patients there was a significant increase in the level of NO, with a mean increase of $65 \cdot 1(14 \cdot 1) \% 10$ hours after challenge. There was a significant relationship between the size of the LAR and increase in NO $(r=0.75, \mathrm{p}<0.01)$. In patients who had a single early response, which was not significantly different from the early response in dual responders, there was no significant change in NO over the 27 hour period or in histamine $\mathrm{PC}_{20}$. In five patients given a control challenge with methacholine rather than AC and followed over the same period there was no change in NO. Similarly, there was no increase in NO after inhaled histamine in any of the patient groups. We conclude that patients who develop a LAR after AC show an increase in the amount of NO in exhaled air which precedes the fall in lung function, whereas patients who have an isolated early response to allergen do not show any change in NO. Similarly challenge with saline or histamine has no effect on exhaled NO. This provides further evidence that exhaled NO may reflect allergic inflammation in asthmatic airways, and may be a useful marker in monitoring asthma and its response to anti-inflammatory treatment.
Imaging allergen-invoked airways inflammation in atopic asthma in vivo using $\left[{ }^{18} \mathrm{~F}\right]$-fluorodeoxyglucose and positron emission tomography (PET)

IK TAYLOR, AA HILL, M HAYES, C RHODES, T JONES, JMB HUGHES, NB PRIDE, RW FULLER Departments of Respiratory Medicine, Clinical Pharmacology and MRC Cyclotron Unit, RPMS, Hammersmith Hospital; Glaxo Group Research Airways inflammation is a cardinal feature of chronic asthma but is currently only visualisable using bronchial lavage and biopsy. Inflammatory foci can, however, be imaged noninvasively using PET and ${ }^{18} \mathrm{~F}$-labelled fluorodeoxyglucose $\left({ }^{18} \mathrm{FDG}\right.$, a non-metabolisable analogue of D-glucose) to quantify glucose uptake of activated granulocytes. We have investigated the effect of segmental allergen challenge on FDG uptake in the lungs of five mild male asthmatic patients (age 23-32, $\mathrm{FEV}_{1} 71-110 \%$ predicted, $\mathrm{PC}_{20}$ histamine $(\mathrm{H})<16 \mathrm{mg} / \mathrm{ml}$, on inhaled $\beta_{2}$ agonists alone). On day 1 , preallergen $\mathrm{PC}_{20} \mathrm{H}$ was measured. The following day at bronchoscopy $20 \mathrm{ml}$ allergen ( $D$ pteronyssinus or mixed glass pollen, range 100-500 units, median 200) was instilled into the posterior segment of the right upper lobe (RUL, time 0 ). A similar volume of saline was instilled into the posterior segment of the LUL. At variable times following allergen instillation PET scanning was performed with the patients resting supine within the scanner. Following an initial transmission scan, about $3 \mathrm{mCi}$ of ${ }^{18} \mathrm{FDG}$ was injected intravenously. Blood was sampled from the contralateral arm whilst a total of 16 sequential time frames of ${ }^{18} \mathrm{FDG}$ radioactivity (each of $15 \times 7 \mathrm{~mm}$ planes) were acquired over the next 66 minutes. Regions of interest (to permit calculation of the rate of uptake of ${ }^{18} \mathrm{FDG}$ ) were defined anatomically and were drawn manually from the initial transmission scan to incorporate the posterior two thirds of the lung fields from the first four planes above the main carina. Rate of uptake of ${ }^{18}$ FDG $\left(\mathrm{min}^{-1}\right)$ was calculated from Patlak plots in which tissue/plasma count ratio $\left(\times 10^{-4}\right)$ was plotted agsinst the integral plasma counts/plasma counts to obtain a slope; the RUL/LUL [R/L] slope ratios were then calculated. Following completion of the PET scan, post allergen $\mathrm{PC}_{20}$ $\mathrm{H}$ was measured and the change in airway reactivity expressed as the doubling dose (DD) shift. ${ }^{18}$ FDG uptake was significantly increased in all studies in the RUL compared to the LUL: slope RUL $6 \cdot 1-26 \cdot 7 \times 10^{-4} \mathrm{~min}^{-1}$ median $12 \cdot 0 v$ slope LUL $0 \cdot 53-8 \cdot 30$, median $2 \cdot 6$, R/L slope ratios $1 \cdot 3-22 \cdot 6$, median $4 \cdot 0, \mathrm{p}<0 \cdot 02$. Median DD shift was $0 \cdot 8$. Local allergen-invoked airways inflammation can therefore be visualised using ${ }^{18} \mathrm{FDG}$ and PET in vivo in asthma. The cellular localisation of the ${ }^{18}$ FDG signal however remains to be determined.

Human bronchial epithelial cell mediators influence eosinophil and neutrophil adhesion to human endothelial cells in vitro

MM ABDELAZIZ, JL DEVALIA, OA KHAIR, RJ SAPSFORD, RJ DAviES Department of Respiratory Medicine and Allergy, St Bartholomew's Hospital, London Several studies have demonstrated that bronchial epithelial cells can synthesise proinflammatory cytokines such as IL-1 $\beta$ and $T N F \alpha$, which influence the adhesion of in- $N$ flammatory cells to endothelial cells. We have cultured human bronchial epithelial cells and investigated the effect of conditioned medium (CM) obtained from these cells after 24 hours, on eosinophil and neutrophil adhesion to human endothelial cell cultures (HEC) in vitro. HEC were incubated with either CM, medium 199, IL-1 $\beta$, or TNF $\alpha$ for six hours and then cocultured with either purified eosinophils or neutrophils for 0.5 hours. Adhesion was expressed as a percentage of the total number of eosinophils/neutrophils adhering to $\mathrm{HEC}$ at the end of this incubation period. CM increased the adhesion of eosinophils from a control (medium 199) value of $9.3 \%$ (range $8.9-12 \cdot 2 \%$ ) to $28.5 \%$ (range $18.8-32 \%$; $<<0.01$ ) and neutrophils from a control value of $13.9 \%$ (range $9 \cdot 3-26 \%$ ) to $28 \cdot 75 \%$ (range 25-40\%; $\mathrm{p}<0.01)$. Similarly, IL-1 $\beta(10 \mathrm{U} / \mathrm{ml})$ and $\mathrm{TNF} \alpha$ $(500 \mathrm{U} / \mathrm{ml}$ ) increased the adhesion of eosinophils to $23.1 \%$ (range $17 \cdot 3-34.7 \% ; \mathrm{p}<0.01$ ) and $23.7 \%$ (range $20.3-30 \%, \mathrm{p}<0.01$ ), respectively, and adhesion of neutrophils to $50 \cdot 15 \%$ (range $39 \cdot 4-75 \cdot 9 \%$; $\mathrm{p}<0.01$ ) and $62.45 \%$ (range $59.1-80 \% ; \mathrm{p}<0.01$ ), respectively. Treatment of cultures with anti-IL-1 $\beta$ and anti-TNF $\alpha$ significantly at- : tenuated the CM-induced adhesion of both eosinophils and neutrophils to HEC. These results suggest that bronchial epithelial cells, through their ability to generate proinflammatory mediators, are likely to play an important role in the pathogenesis of airway disease by influencing the expression of endothelial cell adhesion molecules which are involved in the adhesion and recruitment of eosinophils and neutrophils. 
Inhibition of GM-CSF induced eosinophil survival and stimulation of DNA fragmentation by cyclic AMP

MP HALLSWORTH, MA GIEMBYCZ, PJ BARNES, TH LEE Department of Allergy and Respiratory Medicine, UMDS, Guy's Hospital, London; Department of Respiratory Medicine, Royal Brompton National Heart and Lung Institute, London GM-CSF has been implicated in the maintenance of eosinophilic inflammation by activating and prolonging the survival of eosinophils (EOS). The signal transduction processes involved in this process are unclear. We investigated the effects of the second messenger cyclic AMP on GM-CSF-induced EOS survival using an in vitro culture assay. The stable, non-hydrolysable analogue of cAMP, dibutyryl cAMP, inhibited EOS survival induced by $\mathrm{GM}-\mathrm{CSF}(1 \mathrm{ng} / \mathrm{ml})$ in a concentration-dependent manner, from a mean (SE) control of 78 (3)\% viability with GM-CSF alone to 20 (4)\% with dibutyryl cAMP $(1 \mathrm{mM})\left(\mathrm{IC}_{50}=200 \mu \mathrm{M}\right)(\mathrm{n}=6)$. Dibutyryl cGMP, included as a control in each experiment, failed to inhibit EOS survival over the same concentration range. Similarly, cholera toxin (CTX) inhibited EOS survival in a concentrationdependent manner from a control of 83 (3)\% viability with GM-CSF alone to $39(10) \%$ with CTX $(1 \mu \mathrm{g} / \mathrm{ml})\left(\mathrm{IC}_{50}=7 \mathrm{ng} / \mathrm{ml}\right)(\mathrm{n}=4)$. The adenylate cyclase activator, forskolin, however, did not inhibit EOS survival at any concentration tested $\left(10^{-8}-10^{-5} \mathrm{M}\right)$. EOS cAMP levels induced by these agents were measured at 24 hours. CTX increased cAMP in a concentration-dependent manner from a control of 59 (12) $\mathrm{fmol} / 10^{6}$ EOS to $515(98) \mathrm{fmol} / 10^{6}$ EOS with CTX $(1 \mu \mathrm{g} / \mathrm{ml})$ $(n=3)$. Forskolin, however, only increased cAMP at the highest concentration tested $(10 \mu \mathrm{M})$ from a control of $69(20) \mathrm{fmol} / 10^{6} \mathrm{EOS}$ with GM-CSF alone to 181 (30) fmol $/ 10^{6} \mathrm{EOS}$ with forskolin ( $\mathrm{n}=$ 5). EOS cultured in the absence of cytokines undergo apoptosis with characteristic fragmentation of their DNA. We have examined the DNA from cells cultured for seven days and found that GM-CSF $(1 \mathrm{ng} / \mathrm{ml})$ prevents this DNA breakdown and the protective effect of GM-CSF can be overcome with the induction of DNA fragmentation by agents that elevate intracellular cAMP ( $1 \mathrm{mM}$ dibutyryl cAMP and $10 \mathrm{ng} / \mathrm{ml}$ CTX)

\section{ICAM-1 expression on alveolar macrophages (AM) is enhanced} by relevant allergen challenge in patients with atopic asthma

M SARNO, S SETHI, J ALLEN, S ROTONDETTO, R KNIGHT, M SPITERI Lung Cell Biology Group, Department of Postgraduate Medicine, Keele University, Staffordshire The intercellular adhesion molecule 1 (ICAM1) has been postulated to play a critical role in the intricate cell-cell interactions leading to the inflammatory response within the lungs of atopic asthmatic patients. In this study, surface ICAM-1 expression was analysed using a monoclonal antibody $\mathrm{RR} 1 / 1 \cdot 1$ (Boehringer Ingelheim, USA) in freshly isolated unstimulated and allergen-stimulated bronchoalveolar lavage cells (BALC) from six atopic asthmatic and six allergic rhinitic patients. A control group of six healthy volunteers was included. Cultures were set up in serum-free RPMI 1640 medium in $35 \mathrm{~mm}$ dishes $\left(1 \times 10^{6}\right.$ cells/well $)$ for 24 hours. The immunostaining intensity was assessed using a semiquantitative visual score from two counts of 300 cells per slide. Baseline expression of surface ICAM-1 in asthmatic patients was preferentially higher within the BAL lymphocyte population. In contrast, no significant ICAM1 staining was detected on either resting lymphoid or monocytoid BAL cells from normal and rhinitic subjects. Stimulation with relevant allergen consistently increased expression of ICAM-1 on asthmatic $A M$, with no change in lymphocyte ICAM-1 expression. In the rhinitic group there was only a slight increase in ICAM-1 expression on AM following relevant allergen stimulation in three out of six subjects. In both asthmatic and rhinitic groups no change was observed with allergens to which the patient was insensitive. These findings support our hypothesis of an allergen-specific immune regulation within the lungs of atopic asthmatics, in which $\mathrm{AM}$ and their enhanced expression of ICAM-1 play a direct functional part.

\section{Spirometric parameters and quality of life in patients with} COPD from the ISOLDE study

C O'LEARY, J DANIELS, PW JONES FOR THE ISOLDE GROUP Division of Physiological Medicine, St. George's Hospital Medical School, London, Department of Respiratory Physiology, Birmingham Heartlands Hospital, Birmingham The relationship between spirometric parameters and impaired quality of life in COPD is weak, but the size of the ISOLDE study (concerning the effect of fluticasone propionate on long-term outcome in COPD) allows more detailed analysis than was possible previously. Spirometry was performed using a computer-controlled rolling seal device (Sensormedics) to ATS quality criteria. This allowed precise detection of the "no-flow" point (i.e. FVC). Spirometry was performed four hours after any previous bronchodilator, then following $400 \mu \mathrm{g}$ salbutamol plus $80 \mu \mathrm{g}$ ipratropium delivered via a Nebuhaler (10 breaths). Baseline data from 273 patients (71 women) were analysed. Their mean (SD) age was 64 (4) years, mean post-bronchodilator $\mathrm{FEV}_{1}$ was 51 (14)\% predicted (\% pred), and total St George's Respiratory Questionnaire (SGRQ) score was 51 (18). Change in $\mathrm{FEV}_{1}$ following bronchodilators $\left(\triangle \mathrm{FEV}_{1}\right)$ was $6 \cdot 7(5 \cdot 3) \%$ pred. Pearson correlations between SGRQ and spirometric values are shown in the table. The correlation between SGRQ score and $\triangle F E V_{1}$

\begin{tabular}{llllll}
\hline & \multicolumn{2}{l}{ Pre-bronchodilators } & & \multicolumn{2}{l}{ Post-bronchodilators } \\
\cline { 2 - 3 } & $r$ & $p$ & & $r$ & $p$ \\
\hline FEV $_{1}$ (\% pred) & 0.14 & 0.022 & & 0.23 & 0.0002 \\
FVC $^{2} \%$ pred) & 0.02 & 0.8 & & 0.07 & 0.25 \\
FEV $_{1}$ /FVC & 0.14 & 0.026 & & 0.20 & 0.0011 \\
\hline
\end{tabular}

(as $\%$ pred) was significant $(r=-0 \cdot 18, \mathrm{p}<0.005)$. In a multiple regression with SGRQ score as the dependent variable both $F E V_{1}$ and $\triangle F E V_{1}$ were significant covariates $(p=0.002$ and $p<0.02$, respectively). The SGRQ score correlated better with the best FEV rather than the worst - probably a result of the standardising effect of bronchodilation. The absence of any correlation between SGRQ and FVC was surprising and may have been due to the use of strict criteria to determine the "no flow" point and ensure the highest possible FVC. This suggests that the component of vital capacity produced in the later part of a long forced exhalation does not influence life quality in patients with COPD.

Treatment of breathlessness without incurring sedation or respiratory depression in anxious patients with COPD using Buspirone

AK DATTA, $S$ MUZZIN, N GENE-COS, $S$ MOOSAVI, $K$ MACRAE, P JONES, A GUZ Departments of Medicine, Charing Cross $\mathcal{E}$ Westminster Medical School, London; St George's Hospital Medical School, London Breathlessness in patients with COPD who maintain near normal aterial blood gases at rest has been linked to anxiety in those patients. Twelve clearly defined non-obese patients with severe breathlessness, severe anxiety and COPD with near normal arterial blood gases at rest have been examined before and after one month's treatment with a new anxiolyic, Buspirone (BUSP; $30 \mathrm{mg} /$ day, $\mathrm{n}=7$ ) or placebo $(n=5)$ in a randomised, double blind trial. Recruitment to the study revealed a high incidence of consumption of psychotropic medication by these patients that had previously been unrecognised by their respiratory physicians. Treatment with BUSP was anxiolytic; there was no effect of placebo on anxiety. Fletcher and oxygen cost diagram scores of breathlessness confirmed severe breathlessness on entry to the study. Following BUSP there was a (statistically nonsignificant) $10 \%$ reduction in breathlessness using either score; following placebo there was a mixed response. No significant difference was seen in the change in overall ventilation, pattern of breathing or breathlessness during hypercapnia between the BUSP and placebo groups. There was no change in the slope or intercept of the ventilation: $\mathrm{PETCO}_{2}$ relation following BUSP or placebo. Sleep, breathing during sleep, and vigilance were unchanged after BUSP. Exercise capacity was measured with a self-paced six minute treadmill test. The data were analysed after normalisation and adjustment for differences in baseline pre-exercise values and for differences in oxygen consumption during the test. No significant difference was seen between the two groups in the total distance walked. There was an increase in oxygen consumption, heart rate and carbon dioxide production during exercise following BUSP compared with placebo. The increase in oxygen consumption was statistically significant. Following BUSP there was a slower respiratory rate during exercise but the overall minute ventilation was preserved. There was a greater level of exercise related oxygen desaturation following BUSP than following placebo. However, this additional desaturation was not observed when adjusted for the increased oxygen consumption seen during exercise following treatment with BUSP. During exercise breathlessness was significantly increased following BUSP when related to oxygen consumption; breathlessness was reduced in relation to ventilation. The results suggest that, at anxiolytic doses, BUSP has no deleterious effect on 
ventilation at rest or when stimulated by hypercapnia or exercise. There is a modest but statistically insignificant reduction in perceived breathlessness at rest. During exercise patients appeared to tolerate a greater level of metabolic work and exercise related oxygen desaturation.

\section{Magnetic stimulation of the diaphragm in patients with chronic hyperinflation}

MI POLKEY, D KYROUSSIS, SEJ KEILTY, GH MILLS, CH HAMNEGARD, M GREEN, J MOXHAM King's College Hospital and Royal Brompton Hospital, London Patients with COPD have hyperinflation and shortening of the diaphragm. This should theoretically result in reduced force and pressure generating capacity, but other investigators (Similowski et al. N Engl f Med 1991;325:917-23) have found evidence of better than expected contractility suggesting a process of chronic adaptation. We therefore used cervical magnetic stimulation of the phrenic nerve roots to study the effect of chronic hyperinflation on diaphragm contractility in seven well trained men with severe COPD (mean $\mathrm{FEV}_{1} 0.641$, range $\left.0.5-0.9\right)$. Lung volumes were determined using whole body plethysmography. To avoid diaphragm potentiation ( $\mathrm{Am}$ $\mathcal{F}$ Respir Crit Care Med 1994;149:739-43) the subjects breathed quietly for 20 minutes after passage of oesophageal and gastric balloon catheters. Twitch transdiaphragmatic pressure (Tw PDI) was then measured at resting end expiration. Five measurements were made for each subject. Tw PDI was reproducible for each subject (coefficient of variation $2 \cdot 7-7 \cdot 5 \%$ ). The mean Tw PDI was $20.3 \mathrm{~cm} \mathrm{H}_{2} \mathrm{O}$ (range 14.9-25) at a mean lung volume of $2 \cdot 1$ litres (range $0 \cdot 9-3 \cdot 2$ ) above predicted FRC. In normal male subjects in our laboratory ( $A m \mathcal{F}$ Respir Crit Care Med 1994;149:A133) the mean Tw PDI at FRC was $31 \cdot 8 \mathrm{~cm} \mathrm{H}_{2} \mathrm{O}$ (range $26 \cdot 5-38 \cdot 1$ ), but at $2 \cdot 1$ litres above FRC the mean Tw PDI was $20.5 \mathrm{~cm} \mathrm{H}_{2} \mathrm{O}$. We conclude that during chronic hyperinflation diaphragm contractility is comparable to that seen in normal subjects during acute hyperinflation and we observed no evidence of chronic adaptation.

\section{Measurement of maximal exercise capacity in patients with severe chronic obstructive pulmonary disease (COPD)}

RS MATHUR, SM REVILL, DD VARA, R WALTON, MDL MORGAN Department of Respiratory Medicine, Glenfield Hospital, Leicester In normal subjects treadmill exercise usually produces the greatest maximal oxygen consumption $\left(\dot{\mathrm{V}}_{2} \mathrm{max}\right)$. This may not be true in COPD where bicycle exercise, which supports the upper limb muscles, may be better tolerated than unsupported walking on a treadmill. The aim of this study was to determine which mode of exercise produced the best performance in patients with severe COPD. Eight patients with severe COPD (mean $\mathrm{FEV}_{1} 28 \%$ predicted, range 18-36) exercised to a symptom limited maximum on a cycle and on a treadmill on separate days. Cycle workload was increased by 10 watts/minute and the treadmill gradient was increased by $2.5 \%$ alternate minutes, the speed remained constant. $\dot{V o}_{2}$, ventilation $(\dot{V E})$, and heart rate $(H R)$ were measured. Lactate concentration was measured at rest and four minutes after exercise. Breathlessness (BS) and perceived exertion (PE) were rated from modified Borg scores. Full pulmonary function tests were carried out during a screening visit. The mean (SE) $\dot{\mathrm{Vo}}_{2}$, $\dot{V} \mathrm{E}$ and HR at peak cycle exercise were $11 \cdot 8(1 \cdot 1) \mathrm{ml} / \mathrm{min} / \mathrm{kg}, 27 \cdot 7(3 \cdot 2) 1$ $\mathrm{min}$, and $120(5 \cdot 5) \mathrm{bts} / \mathrm{min}$ respectively, and for peak treadmill exercise $12 \cdot 0(1 \cdot 1), 26 \cdot 0(2 \cdot 8)$, and $121(7 \cdot 4)$. There were no significant differences, though individual variability was large. There was a difference between the exercise lactate concentrations, with cycle exercise generating the greatest response $2 \cdot 49(0.42)$ v $1 \cdot 22(0.27) \mathrm{mmol} / \mathrm{l}$, $(p<0.05)$. There was a better correlation between the Borg scores and $\dot{\mathrm{Vo}}_{2}$ during cycle exercise than during treadmill exercise $(0 \cdot 700$ for BS and 0.784 for PE $v 0.448$ and 0.495 ). The relationships between static lung function and exercise performance were poor. There was no clear difference between the two forms of exercise. Individual variation was large therefore the best performance cannot be predicted. This can only be determined if both types of exercise are carried out. Cycle exercise was unfamiliar to the patients and generated the greatest lactate response. The results do not support the hypothesis that cycle exercise consistently produces a better performance in severe COPD. [RSM sponsored by Raj Nanda Pulmonary Disease Research Trust Delhi, and The British Thoracic Society.]

\section{Metabolic and cardiorespiratory responses to inspiratory} threshold loading in COPD

SM REVILL, MDL MORGAN, AE HARDMAN Department of Respiratory Medicine, Glenfield Hospital, Leicester; Department of Sports Science, Loughborough University The aim of the study was to examine the metabolic and cardiorespiratory responses to inspiratory threshold loading (ITL) in patients with COPD. A solenoid device was used to generate the threshold pressure. Its use has been described in ? normal subjects (Bardsley PA et al. Thorax 1993;48:354-9). Eleven $\overrightarrow{\overline{\vec{D}}}$ patients were recruited $(8 \mathrm{M}$, mean age 61$)$ with moderate COPD (mean (SD) $\left.\mathrm{FEV}_{1} 1 \cdot 29(0 \cdot 43) \mathrm{l}\right)$. All patients carried out a practice test and seven carried out an additional test to determine repeatability. Patients breathed through a mouthpiece attached to the solenoid $\frac{\bar{S}}{D}$

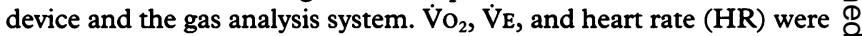
measured at rest, and with mouth pressure $(\mathrm{Pm})$ and the pressure time index ( $\int \mathrm{Pdt}$ ) during ITL. The initial load and size of increments $\mathrm{c}$ were determined for each patient, aiming to achieve a maximum $\vec{\circ}$ within 10 minutes. The load was increased every minute until the patient could no longer inspire. A blood sample was taken at rest and $\vec{\omega}$ four minutes after loading to determine lactate concentration. The repeatability of peak $\mathrm{Pm}$ and $\int \mathrm{Pdt}$ at the end of the test was good but $\vec{x}$ the $\dot{\mathrm{Vo}}_{2}$ peak and $\dot{\mathrm{V}}$ e peak were more variable. The mean difference 0 and $95 \%$ limits of agreement (LA) for tests 2 and 3 are shown in the table. For the whole group $(n=11)$ the mean $(S E)$ peak Pm was

\begin{tabular}{|c|c|c|c|c|}
\hline Test & $\begin{array}{l}\text { peak } \mathrm{PM} \\
\left(\mathrm{cm} \mathrm{H}_{2} \mathrm{O}\right)\end{array}$ & $\begin{array}{l}\int P d t \\
\left(\mathrm{~cm} \mathrm{H} \mathrm{H}_{2} \mathrm{O} / \mathrm{s}\right)\end{array}$ & $\begin{array}{l}\dot{\mathrm{V}} \mathrm{O}_{2} \\
(\mathrm{ml} / \mathrm{min})\end{array}$ & $\begin{array}{l}\dot{V}_{E} \\
(\text { (l/min) }\end{array}$ \\
\hline $2 v 3$ & $\begin{array}{c}-0.4(2.3) \\
-12.8 \text { to } 11.9\end{array}$ & $\begin{array}{c}110(201 \cdot 5) \\
-956.3 \text { to } 1176 \cdot 3\end{array}$ & $\begin{array}{c}47(36 \cdot 1) \\
-144 \cdot 1 \text { to } 238 \cdot 1\end{array}$ & $\begin{array}{c}2.3(1.7) \\
-6.7 \text { to } 11.3\end{array}$ \\
\hline
\end{tabular}

$52(7 \cdot 0) \mathrm{cm} \mathrm{H}_{2} \mathrm{O}, \int \mathrm{Pdt} 1410(261) \mathrm{cm} \mathrm{H}_{2} \mathrm{O} / \mathrm{s}, \dot{\mathrm{VO}}_{2}$ peak $367(19 \cdot 7) \mathrm{ml} / \stackrel{\mathrm{O}}{\mathrm{O}}$ min, VE peak $24 \cdot 2(1 \cdot 9) 1 / \mathrm{min}$, and HR peak $89(5 \cdot 7)$. The mean (SD)

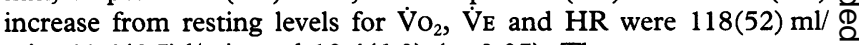
min, $11 \cdot 4(6.5) 1 / \mathrm{min}$ and $12 \cdot 4(6 \cdot 0)(\mathrm{p}<0.05)$. These represent mean percentage increases of 47,96 , and 16 , respectively. There was no $ㅇ$ significant difference in lactate concentration. Threshold loading in COPD using the solenoid device was reproducible. The incremental, progressive protocol produced significant increases in $\dot{\mathrm{Vo}}_{2}, \dot{\mathrm{VE}}$ and HR, but no lactate response.

Bicycle ergometer underestimates symptom-limited maximal $\mathrm{V}_{2}$ in subjects with moderate chronic obstructive pulmonary disease (COPD): implications for clinical testing and rehabilitation programmes

AN MCLEAN, LM COCHRANE, CJ CLARK Hairmyres Hospital, East Kilbride, $\frac{D}{2}$ Lanarkshire Cycling and walking are used as means of assessing exercise capacity and exertional dyspnoea and to improve exercise $\mathbb{N}$ tolerance in pulmonary rehabilitation programmes. The choice of exercise mode is often arbitrary. The systematic differences in sub- 0 jective and physiological responses between the two modes in subjects $\mathrm{N}$ with COPD are not known. We examined the effect of each mode of $\sigma$ exercise on the relationships of: (1) breathlessness (Borg rating) with oxygen uptake $\left(\dot{\mathrm{VO}}_{2}\right) ;(2)$ minute ventilation $(\mathrm{VE})$ with $\mathrm{Vo}_{2}$; and (3) $\dot{\mathrm{V}} \mathrm{E}$ with Borg. We also compared symptom limited maximal $\mathrm{Vo}_{2} \stackrel{\mathbb{P}}{+}$ between modes in subjects with COPD. Nine subjects with moderate COPD (mean $\mathrm{FEV}_{1} 45 \%$ pred) performed a symptom-limited pro- $\frac{}{D}$ gressive incremental test on bicycle (B) and treadmill (TM) on the $\frac{\mathbb{D}^{\circ}}{\square}$ same day. Regression analysis was carried out for each subject for $\frac{\rho}{\rho}$

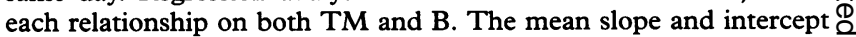
between the modes was compared using the paired $t$ test. For Borg $v \dot{\mathrm{VO}}_{2}$ there was no significant difference, slope TM $0.0098 v \mathrm{~B}$ $0 \cdot 0088$, constant $\mathrm{TM}-6 \cdot 25 v \mathrm{~B}-5 \cdot 36$. For $\dot{\mathrm{VE}} v \dot{\mathrm{Vo}}_{2}$, $\dot{\mathrm{V} E}$ was lowero on the TM, slope $0.026 v 0.030$ ( $\mathrm{p}<0.01$ ), constant $3.88 v 3.30$. For Borg $v$ VE, Borg was higher on TM, slope $0.328 v 0.274(\mathrm{p}<0.01)$, constant $-5.28 v-6.75$. Maximal $\mathrm{Vo}_{2}$ was higher on TM, $1.53 v ?$ $1.29(\mathrm{p}<0.001)$ but there was no significant difference in maximal $\dot{V E}$ $(46.2 v 44 \cdot 1 \mathrm{l} / \mathrm{min})$. Subjects were working close to their predicted maximal voluntary ventilation in both modes with dyspnoea indices of $89 \% v 91 \%$ for B and TM respectively (NS). This study shows that less work was achievable on the bike which may significantly underestimate symptom-limited maximal $\mathrm{Vo}_{2}$ in these patients. This has not been previously described in this group of subjects in whom 
the respiratory limitation might be expected to predominate and prevent differences in maximal work capacity from appearing. The submaximal data show a difference in ventilatory response between the two modes but suggest that subjective breathlessness remains closely linked to $\mathrm{VO}_{2}$ irrespective of the mode of exercise.

\section{Outcomes in occupational asthma}

DJ ROSS, JC MCDONALD SWORD Project, National Heart and Lung Institute, London SWORD (surveillance of work-related and occupational respiratory diseases) is a voluntary reporting scheme in which $83 \%$ of consultant chest physicians in the UK participate as well as a similar number of occupational physicians. We have undertaken a study of the long term respiratory health and employment after a diagnosis of occupational asthma. A total of 1940 cases of occupational asthma in the age group 16-65 were notified to the scheme from 1989-1992. A questionnaire was sent for each case to the 312 physicians who reported them. Duplicates, cases where the physician had retired, or the diagnosis had been revised were excluded. So far, $81 \%$ of questionnaires have been returned, 792 by chest physicians (35\% initially medicolegal) and 231 by occupational physicians ( $4 \%$ medicolegal). Time from notification to response was $17-68$ months. 750 cases were not medicolegal. Of these, $21 \%$ were no longer considered to have asthma, $74 \%$ had improved, $22 \%$ were unchanged, and $4 \%$ were worse; $48 \%$ were with the same employer and $13 \%$ were employed elsewhere, $23 \%$ were unemployed, $7 \%$ medically retired or on chronic sick leave, and $9 \%$ retired. Unskilled workers had a higher probability of leaving their employer than skilled workers. The mean duration of exposure to the suspected agent was 20.0 months in those who recovered from asthma, but was 52.6 months in those still affected. The mean duration of exposure after diagnosis was 5.7 months in those who recovered, but 11.0 months otherwise.

\section{Work-related symptoms and specific sensitisation in a cohort} of workers exposed to acid anhydrides

RD BARKER, $M$ VAN TONGEREN, JM HARRIS, KM VENABLES, AJ NEWMAN TAYLOR Department of Occupational and Environmental Medicine, National Heart and Lung Institute, London; Institute of Occupational Health, Birmingham To study exposure response relationships in occupational asthma we have examined a cohort of workers exposed to acid anhydrides (AA) at four industrial sites in the UK. Participants completed questionnaires on respiratory symptoms, smoking habits and work history and had skin prick tests (SPT) with common inhalant allergens and three AA-human serum albumin (AA-HSA) conjugates. In 1992 exposure to phthalic anhydride (PA), trimellitic anhydride (TMA), and maleic anhydride (MA) was measured with personal samplers during full shifts. Past exposure was estimated using job and process descriptions and past exposure measurements. The risk of being sensitised or experiencing new work-related chest symptoms was assessed in relation to full shift AA exposure. In total, 506 workers fulfilled the cohort definition of whom 401 (79\%) took part (388 (97\%) men; age range 18-81, median 39 years). Thirty four (9\%) had new work-related chest symptoms and $12(3 \%)$ had a positive SPT to an AA-HSA conjugate. Average full shift exposure to all AA, measured in 1992, was below the occupational exposure limit at all sites. There was a trend for an increasing prevalence of sensitisation with increasing exposure to TMA ( $\chi^{2}$ for linear trend $\left.p=0.019\right)$. This trend was not apparent when all three AA were included in the analysis. The highest average full shift exposure experienced by five workers sensitised to TMA was less than one quarter of the current UK occupational exposure limit. A matched case-control analysis showed an increased risk of new work-related chest symptoms with increasing estimated full shift exposure to acid anhydrides. The data show that higher full shift exposures are associated with a greater risk of sensitisation and symptoms. They also suggest that the current occupational exposure limit for TMA may be too high.

\section{Coal miners aged over 70 seeking industrial injury benefit for} bronchitis and emphysema

PS SANDHU, SC STENTON, SJ BOURKE, DJ HENDRICK Chest Unit and Regional Unit for Occupational Lung Disease, Newcastle General Hospital, University of Newcastle upon Tyne; Benefits Agency Medical Service Industrial injury benefit for coal miners suffering from chronic bronchitis and emphysema was introduced in September 1993. Miners with at least 20 years underground exposure, an FEV of at least 1 litre below the Cotes predicted values, and pneumoconiosis (ILO category $1 / 0$ or above) are eligible for benefit. To date, over 7300 miners have been evaluated at the Newcastle office of the Benefits Agency. We have collected data on respiratory symptoms, exposure and smoking histories, lung function and radiographic abnormalities on as many of these as possible and have analysed an initial cohort of 1565 workers aged over 70 years. $1383(88 \%)$ of these subjects started work underground before the age of 20 , and their median duration of underground work was 39 years (1-50); $1377(90 \%)$ reported cough and sputum and $1524(99 \%)$ reported breathlessness, $812(52 \%)$ used inhalers, 1145 (75\%) were current or ex-smokers with a median reported cigarette consumption of 10 / day, 514 subjects (34\%) had $\mathrm{FEV}_{1}$ values of more than 1 litre below the predicted value. Of these, $147(29 \%)$ had radiographic evidence of pneumoconiosis and received compensation. The median disability assessment was $50 \%(10-100 \%)$. Stepwise linear regression analysis suggested that $\mathrm{FEV}_{1}$ was positively related to height and was negatively related to age, the use of inhalers, smoking, and the presence of cough. There was a slight but significant $(\mathrm{p}<0.001)$ positive relationship with years of underground work with $\mathrm{FEV}_{1}$ increasing by 0.0071 per year of underground exposure.

Work-related symptoms and specific sensitisation in two occupational cohorts

P CULLINAN, D LOWSON, M NIEUWENHUIJSEN, S GORDON, C SANDIFORD, RD TEE, KM VENABLES, JC MCDONALD, AJ NEWMAN TAYLOR Department of Occupational and Environmental Medicine, National Heart and Lung Institute, London The development of symptoms and specific sensitisation over up to five years has been compared in two newly exposed occupational cohorts; incident, work-related respiratory, eye/nose or skin symptoms and positive, specific skin prick tests (SPT) were related to measured whole shift exposures to aeroallergen or dust in 208 laboratory animal workers and 103 flour exposed workers. In both cohorts the incidence of all symptom types was highest in the first year of exposure; eye/nose symptoms were most commonly reported. Amongst flour workers there was no evidence of a relationship between new symptoms and a positive SPT to flour; in laboratory workers with specific sensitisation and symptoms these tended to be identified simultaneously. In the flour workers an exposure response relationship was seen only with exposure expressed in terms of dust; there were no associations with atopy or smoking. In laboratory employees regression analysis revealed an association between eight hour rat urinary aeroallergen exposure and new skin $\left(\mathrm{OR}=1.12\right.$ per $\left.10 \mu \mathrm{g} / \mathrm{m}^{3}\right)$ and eye/nose symptoms $(\mathrm{OR}=1.01)$, and the development of a specific SPT (OR=1.13); smoking and atopic status were also associated with these outcomes. These findings are consistent with those from an initial cross sectional analysis and suggest that the development of work-related disease in these two groups may reflect different pathological processes or selection pressures.

Comparison of across shift and across week variations in lung function in symptomatic textile workers and matched controls

CJ WARBURTON, AM FLETCHER, CAC PICKERING, RM NIVEN, LA OLDHAM, HC FRANCIS Northwest Lung Centre, Wythenshawe Hospital, Manchester Eighty four textile workers with one or more respiratory symptoms and 84 asymptomatic control workers (defined by questionnaire) performed spirometric and bronchial reactivity (BR) testing at the beginning and the end of the first and fourth shifts of the working week. The symptomatic workers were significantly older than the controls, and demonstrated lower levels of percent predicted lung function ( $p<0.05$ only for $\mathrm{FEF}_{25-75}$ ). There was no difference between the groups for current or past cotton dust exposure. On day 1 both groups demonstrated falls in spirometric values and increases in BR across the shift. None of these differences was significant. On day 4 both demonstrated a fall in $\mathrm{FEV}_{1}$ and FVC with rises in $\mathrm{FEF}_{25-75}$ and $\mathrm{FEF}_{75-85}$. There was a significant increase in BR in the controls as opposed to the symptomatics on this day $(p<0.05)$. The symptomatic workers demonstrated significantly greater falls in $\mathrm{FEV}_{1}$ and FVC across the working week than the controls. There was little difference for $\mathrm{FEF}_{25-75}$ and $\mathrm{FEF}_{75-85}$. Exposure to textile dust seems to have similar effects upon workers with and without symptoms, and this effect is more marked on the first working day. Symptomatic operatives 
do not recover lung function as quickly as the controls leading to declines across the working week. Asymptomatic workers seem to experience the largest changes in $\mathrm{BR}$.

\section{Are the respiratory health effects seen in ceramic fibre man-} ufacturers due to the dust rather than the fibre exposure?

PS BURGE, WN TRETHOWEN, JM HARRINGTON, IA CALVERT Birmingham Heartlands Hospital and Institute of Occupational Health, Birmingham University, Birmingham We have carried out a cross sectional survey of all seven European ceramic fibre manufacturing plants, estimating current exposure using full shift personal samplers, measuring inspirable dust and respirable fibre levels. The response rate was $89 \%$ of 708 current employees. Plant and job specific cumulative exposure levels were calculated from detailed occupational histories based on current exposure estimates. Respiratory health was measured by questionnaire and spirometry. Odds ratios relating current symptoms to current exposures were calculated by multiple logistic regression and regression coefficients for lung function related to cumulative exposure were calculated using multiple linear regression, each controlling for the effects of respirable fibre and inspirable mass exposures separately and together, after adjusting for age, sex, plant (and hence country) and smoking. Significantly increased odds ratios for current exposure to both dust and fibres were seen for dry cough, stuffy nose eye and skin irritation and breathlessness. There were significan decrements in $\mathrm{FEV}_{1}$ and $\mathrm{FEF}_{25-75}$ seen in current smokers which were related to cumulative fibre exposure but not cumulative dust exposure. No effect was seen in lifelong non-smokers, intermediate effects were seen in ex-smokers. In conclusion, current symptoms were related to both inspirable mass and respirable fibre exposures. Dose-related decrements in lung function related only to cumulative respirable fibre exposure and were confined to smokers, suggesting a promoting effect of ceramic fibres on the effects of cigarette-related declines in $\mathrm{FEV}_{1}$.

Scottish national survey of tuberculosis notifications in 1993 with special reference to the prevalence of HIV seropositivity

AG LEITCH, M RUBILAR, GI FORBES, J CURNOW, G BOYD, $s$ BURNS, B WATt Tuberculosis Survey Study Office, Royal Victoria Chest Clinic, Chalmers Hospital, RIE NHS Trust, Edinburgh All notifications of tuberculosis in Scotland in 1993 were surveyed by questionnaire and a $100 \%$ response rate was achieved by supervising clinicians. HIV testing was requested for under 65 year olds. Of 497 notifications, $423(85 \%)$ were white and $58(12 \%)$ were from the Indian subcontinent (ISC). Of those from the ISC $85 \%$ were aged under 55 years whereas $64 \%$ of white patients were aged over 55 years. $37 \%$ of white and $47 \%$ of ISC patients were male. Pulmonary disease was found in $74 \%$, non-pulmonary in $22 \%$, and combined disease in $4 \%$ of patients. ISC $(47 \%)$ had more non-pulmonary disease than white (15\%) patients. Genitourinary disease (33\%) and pleural effusion $(17 \%)$ were commoner in white patients and lymphatic disease $(67 \%)$ in ISC patients. Cultures for tuberculosis were positive in $64 \%$ of white and $65 \%$ of ISC pulmonary cases with $48 \%$ and $45 \%$ positive smears. Of $244 \mathrm{HIV}$ tests performed three, including one previously known, proved positive. Five other HIV positive patients were known, giving an HIV positivity rate of $1.6 \%$ for the 497 Scottish tuberculosis notifications in 1993. Four HIV positive patients were African. The annual tuberculosis notification rate for Scotland in 1993 was 9.7/ $10^{5}$. In white patients the rate was $8 \cdot 4 / 10^{5}$ with higher rates for men and increasing rates with age in both sexes. The ISC population rate for Scotland was $179 / 10^{5}$. Notification rates for white patients were higher in Glasgow $\left(13 / 10^{5}\right)$ than in Edinburgh $\left(6 \cdot 9 / 10^{5}\right)$. Tuberculosis notifications in Scotland have fallen by $5 \%$ since 1992 . HIV infection is an uncommon accompaniment of tuberculous disease in Scotland.

\section{Changing pattern of respiratory tuberculosis in adult patients} from the Indian subcontinent

OR MCCARTHY, LP ORMEROD Newham Chest Clinic, London; Blackburn Royal Infirmary, Blackburn, Lancashire Clinical observation over 10 years in both clinics in high tuberculosis prevalence areas suggested an altering pattern of respiratory tuberculosis in adult patients from the Indian subcontinent (ISC), with a declining proportion of isolated mediastinal lymphadenopathy, and an increasing proportion of smear positive and culture positive disease. Details of all 878 adult ISC (age $>15$ ) cases treated in the two centres in the years 1981-92 were analysed. The main site of disease, sputum status at diagnosis, time since first entry to the UK, ethnic group, and sex were known for all patients. General logistic regression showed a highly significant effect by year $(p=0.0002)$. There was a highly significant trend over time $(\mathrm{p}=0.0002)$, with an increasing percentage culture positive, but no difference between centres $(p=0.65)$ or centre by year interaction $(p=0 \cdot 47)$. There was also a significant trend to decreasing percentage mediastinal lymphadenopathy over time $(p=0.002)$, with no centre by year interaction $(p=0.90)$ but a difference between the centres $(p=0.02)$. Analysis of other factors to attempt to explain the increased culture positive disease and decreased mediastinal glands, particularly since 1987, showed no change in the proportions of respiratory and non-respiratory disease, and no correlation with the recognised risk factors of duration since first UK entry and return visit to the ISC. The change from 1987 onwards may therefore be due to social deprivation which has increased since 1987.

Results of the national survey of tuberculosis notifications in England and Wales in 1993

JM WATSON ON BEHALF OF A PHLS/BTS/DH COLLABORATIVE GROUP PHLS Communicable Disease Surveillance Centre, London A survey of tuberculosis notifications in England and Wales in 1993 was carried out to determine the occurrence of the disease in the whole population and in population subgroups. Detailed information was obtained in the first six months of the survey on site of disease and bacteriological results. The prevalence of HIV infection in adults (16-54 years) notified over the whole year was estimated using unlinked anonymous HIV testing. By 30 September 1994, for 2338 eligible notifications in the first six months, $1997(85 \%)$ clinical forms had been received. Of these, $41 \%$ patients were white, $43 \%$ from the Indian subcontinent (ISC), and $16 \%$ from other ethnic groups (the equivalent figures in the 1988 survey were $53 \%, 39 \%$, and $8 \%$, respectively). After exclusion of previously treated cases and allowing for incomplete data, the crude annual notification rate for England was $8 \cdot 7$ per 100000 compared with 8.6 in 1988 . The rates in the white and ISC ethnic groups were 3.9 and 115.6 per 100000 compared with 4.7 and 120 in 1988 , respectively. Bacteriological results were available for $1029(88 \%)$ of the 1169 pulmonary cases: $659(64 \%)$ were positive on culture; 574 $(56 \%)$ had positive smears. Of the 659 with positive cultures 27 $(4 \cdot 1 \%)$ were resistant to one or more first line drugs, including 12 $(1 \cdot 8 \%)$ resistant to isoniazid alone and a further $13(2 \cdot 0 \%)$ to isoniazid in combination with other drugs. Specimens were received for 1073 ( $40 \%$ ) of the 2701 patients eligible for inclusion in the HIV prevalence survey: $22(2 \cdot 1 \%)$ were positive. The continued decrease in white and ISC ethnic group specific rates suggest that changes in the demographic structure of the population and the increased number of cases in the other ethnic groups have contributed to the overall increase in notifications.

\section{UK mycobacterium resistance network}

D BeNnet, J WATSON, D KUMAR, M YATES, T JeNkINS PHLS Communicable Disease Surveillance Centre (CDSC); Dulwich Public Health Laboratory; Mycobacterium Reference Unit In order to characterise drug resistance trends for Mycobacterium tuberculosis isolates more frequently than under the five yearly national surveys, the PHLS Mycobacterium Reference Unit and Regional Tuberculosis Centres have agreed to report information on mycobacterial isolates they receive for UK residents to CDSC. The reference laboratories in Scotland and Northern Ireland, as well as the Brompton Hospital laboratory, are also participating. An electronic system for regular reporting of the relevant demographic, geographical, specimen site, sputum microscopy, and antibiotic susceptibility information, based on an agreed minimum data set, has been piloted since January 1994 . Reports are made on all primary isolates and isolates in which susceptibility has changed. A preliminary analysis of 643 deduplicated reports received on $M$ tuberculosis isolates through April 1994 indicates that resistance to isoniazid and rifampicin combined could be rising in England and Wales: combined resistance was $1.8 \%$ compared with a preliminary estimate of $0.8 \%$ from the 1993 National Survey of Tuberculosis Notifications in England and Wales, and $0.6 \%$ from the 1988 survey. Increases were also seen in other resistance measures. Resistance patterns by disease site did not differ significantly among ethnic groups, except that significantly more pulmonary isolates as. . .

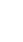
. .

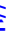
.

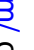
. .

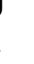

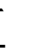
. , 
sociated with African ethnic origin were resistant. SOUNDEX code and date of birth matched for 29 isolates (4.5\%) to the CDSC HIV data bases. Unexpectedly, all measures of resistance were significantly higher among these isolates: resistance to at least one first line drug was found in $27.6 \%$, compared with $9.1 \%$ among those not known to be HIV seropositive. Complete reporting from all centres over a longer period is needed before definite trends can be discerned. Analyses will be carried out for reports through September 1994.

Relative diagnostic value of gastric lavage, bronchoalveolar lavage, and sputum examination in the diagnosis of pulmonary tuberculosis: a retrospective survey

BG MARSHALL, RN DAVIDSON, RA WALL, GV PASVOL Departments of Infection and Tropical Medicine and Microbiology, St Mary's Hospital Medical School, Northwick Park Hospital, Harrow, Middlesex We treat over 100 patients with tuberculosis (TB) each year, arising from the local population (mostly Asians) as well as cases referred from the Port Health Authority at Heathrow. We routinely examine specimens of gastric lavage (GL), sputum and bronchoalveolar lavage (BAL) specimens in the diagnostic work up for pulmonary TB. A retrospective review was undertaken to assess the relative efficiency of each of these investigations going back over a total of 20 months of microbiological records. Approximately 2600 samples were cultured for mycobacteria during this period. Sixty six patients had a confirmed diagnosis of pulmonary TB, as defined by one or more positive mycobacterial culture from GL, BAL or sputum. Thirty six of these 66 patients underwent one or more GL; 13 of the 36 (36\%) were smear positive, $28(78 \%)$ were culture positive, eight patients were smear and culture negative, the diagnosis being confirmed by other means. Forty seven patients produced at least one specimen of sputum; $25(53 \%)$ were smear positive, $44(94 \%)$ were culture positive, and three $(6 \%)$ were smear and culture negative. Fibreoptic bronchoscopy was performed on 28 of the 66 patients. Of these, $12(43 \%)$ were smear positive, 25 (93\%) were eventually culture positive, three $(7 \%)$ were culture negative. BAL provided the sole positive culture in five of $66(7 \cdot 6 \%)$ patients (and in both of those whom it was the only investigation performed). BAL gave a positive smear or culture result earlier than GL or sputum in five of 66 patients $(7.6 \%)$, whereas GL or sputum produced a time advantage over BAL in four of $66(6 \cdot 1 \%)$ patients. Each of these three investigations plays an important role in the "work up" of patients with suspected pulmonary TB, adding to the diagnostic yield. Each is a sensitive, safe and relatively non-invasive technique. BAL is the investigation of choice when other samples are smear negative, or if the patient is unable to provide them.

\section{Tuberculosis: how to improve notification rates}

JS BROWN, F WELLS, G DUCKWORTH, NC BARNES The London Chest Hospital, London; The Royal London Hospital, Whitechapel, London Tuberculosis (TB) is a statutory notifiable disease. The accuracy of the statistics from notifications is important for the planning of TB services and for thorough contact tracing. However, we have previously shown over a quarter of cases of TB were not notified for the years 1985-9 in an area of East London served by the London Chest and Royal London hospitals (Sheldon et al. Thorax 1992;47:1015-8). As a result several changes in practice were instigated to improve notification rates: clinicians were encouraged to refer all cases of TB to the chest physicians for advice on management; suggestive histology reports and all positive microbiology reports were sent to the communicable disease control (CDC) consultant to check whether the patient had been notified or not; and pharmacy informed the CDC consultant about patients prescribed isoniazid. We now report a repeat audit of TB notification rates for the years 1992-3 from these two centres. Identical methods were used to identify cases as the original study, and comparisons were made between the two sets of data. 252 cases of adult TB were identified for the years 1992-3, giving an annual incidence of 126 cases/year which is similar to the original study (122/year). The mean age was 46 years and 148 (59\%) cases were male. There was a significant increase in the proportion of patients of African origin from 15 out of $580(3 \%)$ in the original study to 29 out of $252\left(12 \%, \chi^{2} 29.5, \mathrm{p}<0.00001\right)$ for cases diagnosed in 1992-3. Ten patients (4.0\%) were known to be HIV positive in the $1992-3$ cohort whereas only four patients $(0.7 \%)$ were known to be HIV positive in the 1984-9 group. 236 cases (94\%) were notified, a highly significant increase of $21 \%$ over the original study where $73 \%$ of cases had been notified $\left(\chi^{2} 42 \cdot 9, \mathrm{p}<0.00001\right.$, confidence intervals $16 \%$ to $25 \%$ ). Only one smear positive patient was not notified. We have therefore shown that changes in the practice of notification for TB can lead to a substantial improvement in notification rates and so provide accurate data for contact tracing and on the local incidence of TB.

Randomised trial of five classes of anti-asthma medication: one month results

A MORICE, M TAYLOR Department of Medicine and Pharmacology, University of Sheffield, Royal Hallamshire Hospital, Sheffield We have compared the effectiveness of five classes of currently prescribed medication in a randomised study in a hospital asthma clinic. Ninety eight patients aged 16-65 years, who were naive to therapy apart from occasional bronchodilators, were recruited into an open trial following informed consent. The diagnosis of asthma was established by either a $15 \%$ diurnal variability in PEFR or a $>15 \%$ bronchodilation to inhaled salbutamol. Patients were randomised to one of the following drug classes: a short acting $\beta_{2}$ agonist (Aerolin Autohaler $200 \mu \mathrm{g}$ prn or Bricanyl turbohaler $0.5 \mathrm{mg}$ prn); a long acting $\beta_{2}$ agonist (Serevent $50 \mu \mathrm{g} \mathrm{bd}$ ); an inhaled steroid (Becotide $200 \mu \mathrm{g}$ bd or Pulmicort Turbohaler $100 \mu \mathrm{g}$ bd); nedocromil sodium $4 \mathrm{mg}$ qds; and oral theophylline (Nuelin $250 \mathrm{mg}$ bd or Theodur $300 \mathrm{mg} \mathrm{bd}$ ). Theophylline levels were monitored to achieve levels of $10-20 \mathrm{mg} / \mathrm{l}$. Symptoms and daily PEFR measurements were recorded in a diary card. Baseline characteristics of the five groups were similar: mean $\mathrm{FEV}_{1}$ (predicted) 2.61 (82\%), FVC 3.61 (91\%). Wheeze, cough and expectoration were present on $4.2,3.8$, and 2.8 days per week. At one month the greatest improvement in number of symptom-free days was seen in the group given inhaled steroids. Mean days per week with wheeze fell by $1 \cdot 3$, cough by 0.5 , and expectoration by 1.5. Nedocromil sodium produced similar but less striking results of $0 \cdot 8,0.3$, and 0.8 respectively. Other modalities of treatment produced no significant change in symptoms. In this group of mild asthmatics mean improvements in $\mathrm{FEV}_{1}$ was greatest in the steroid group $(11 \%)$ followed by the nedocromil $(9 \%)$ and salmeterol $(7 \%)$ groups. There was no change with short acting $\beta_{2}$ agonist or theophylline. Antiinflammatory therapy produces the greatest symptomatic and physiological improvement in mild asthma. [Supported by Astra, Fisons, Allen \& Hanburys, and 3M.]

Patterns of prescribing of inhaled steroids over a seven year period in a general practice and its implications

DB PRICE Thorpewood Surgery, Woodside Road, Norwich There is evidence that the prevalence of asthma appears to be increasing (Burr et al. Arch Dis Child 1989;64:1452-6). As asthma increases so does the burden of asthma costs. It is recommended that an essential part of the management of asthma is the use of inhaled steroids. An audit was carried out in my practice to look at the increase in prescribing costs and its relation to both the drugs used and the costs per patient. Between 1987 and 1994 the recorded asthma population in my practice of 10500 patients has increased from 345 to $1106(10 \cdot 5 \%)$. A variety of factors may be responsible: increasing diagnosis and prevalence, increasing practice population (13\%), changing local population, and asthmatics changing practices $(30 \%$ of transferring patients). Asthma prescribing costs have more than doubled over the study period from $£ 39706$ in $1987-8$ to $£ 89040$ in $1993-4$. This consists mainly of spending on inhaled steroids which has risen from $£ 14374$ to $£ 53640$ with a small increase in bronchodilator costs and falls for other asthma medications. The number of asthmatics receiving inhaled steroids has increased from 110 to 694 . Thus, annual drug costs per diagnosed asthmatic have fallen from $£ 115$ to $£ 80$ with inhaled steroids staying between $£ 40$ and $£ 48$. Clinical improvements have been found in terms of reduced total admissions for asthma over the age of three $(43 \%)$ and outpatient attendances $(65 \%)$ over the period. For individual asthmatics changed to inhaled steroids the decrease is $74 \%$ and $90 \%$ respectively. Total recorded exacerbations fell by $25 \%$ in the last two years. The data in this study support the premise that increased use of inhaled steroids in the management of asthma can be cost effective.

Effects on bone metabolism of inhaled beclomethasone dipropionate (BDP) with and without oral prednisolone in asthmatic men compared with controls

EN EVANS, G MORRIS, $s$ woodhead, P EBDEN Prince Philip Hospital Dafen Llanelli; University Hospital of Wales, Heath Park, Cardiff We 
have studied serum osteocalcin and serum parathyroid hormone (PTH) pre and post oral calcium load (1200 mg calcium) in 10 male asthmatic patients (mean age 52.7 years) taking inhaled BDP (mean dose $1000 \mathrm{mg}$ per day) and 10 asthmatic male patients (mean age 55.6 years) on inhaled BDP with oral corticosteroid mean daily dose prednisolone $9.75 \mathrm{mg}$ (P.) These groups were compared against 10 aged matched male controls (mean age 54.4 years) on no corticosteroid preparation (C). Subjects on steroid preparations had been taking their medication for at least three months with no change (P 0.25-10 years, BDP 0.25-7 years). All subjects had normal renal and bone biochemistry. Osteocalcin levels were all in the normal range and there was no difference statistically between groups (C $5.55(1.26) \mathrm{ng} /$ $\mathrm{ml}$; P 4.31 (1.57); BDP 5.74 (3.22)). PTH levels were all in the normal range pre calcium load (C $4.54(1.30) \mathrm{pmol} / \mathrm{ml} ; \mathrm{P} 4.16(0.86)$; BDP 4.04 (1.28)). PTH decrement after calcium load (C 2.02 $(1.15) \mathrm{pmol} / \mathrm{ml}$; P $1.81(0.75)$; BDP $1.64(0.76))$ were all in the normal range and not statistically different. Both patient groups with chronic stable corticosteroid use and controls did not have different osteocalcin levels, PTH levels and response to calcium absorption. These findings indicate stable bone biochemistry in patients with chronic stable steroid ingestion and suggest interpretation of findings of acute studies should be made with caution.

Inhaled corticosteroids, asthma, and longer term bone metabolism: collagen crosslinks, osteocalcin, and densitometry after one year

K ANDERSON, SJ GALLACHER, WD FRASER, T SPEEKENBRINK, D VERNON, IT BOYLE Department of Respiratory Medicine, Western Infirmary; University Department of Medicine, Royal Infirmary, Glasgow Hitherto, most studies of bone metabolism in asthma relating to inhaled corticosteroids have been cross sectional, of short duration, and may have included patients who have taken oral corticosteroids. We compared the effect of inhaled beclomethasone (BDP) $500 \mu \mathrm{g} \mathrm{bd} \mathrm{(} n=5$, mean age 50 years) and inhaled budesonide (BUD) $400 \mu \mathrm{g}$ bd ( $\mathrm{n}=4$, mean age 41 years) on bone metabolism (urinary collagen crosslink excretion, osteocalcin, and lumbar spine/hip density by dual energy $x$ ray absorptiometry) measured pretreatment and after one year in patients with asthma who had never taken oral corticosteroid. The drugs were inhaled through a spacer followed by rinsing the mouth with water in an attempt to reduce non-respiratory drug deposition. Mean (SE) pretreatment L2-4 densities were similar in both groups (BDP $1.162(0.020) \mathrm{g} / \mathrm{cm}^{2} v$ BUD $\left.1.179(0 \cdot 030), \mathrm{p}=\mathrm{NS}\right)$. However, L2-4 density fell significantly over the year after BDP $(-5 \cdot 8(3 \cdot 1) \%$ $v$ BUD $+1.9(1.5) \%, p=0.028)$. No change was noted in femoral neck density. Pretreatment serum osteocalcin was similar (BDP 8.0 $(2.0) \mathrm{ng} / \mathrm{ml} v 8.7(2.0) \mathrm{BUD}, \mathrm{p}=\mathrm{NS})$ and did not change significantly after one year. Pretreatment concentrations of fasting urinary free pyridinoline/creatinine (BDP 12.8 (1.0) $v 15.2(1.9)$ BUD, $\mathrm{p}=\mathrm{NS}$ ) and free deoxypyridinoline/creatinine (BDP $2.5(0.2) v$ BUD 2.9 $(0 \cdot 3), p=N S)$ were not changed with treatment. These results suggest that, although biochemical markers of bone turnover may show no change following treatment with BDP or BUD, lumbar spinal density would appear to fall after one year of BDP. Urinary collagen crosslink excretion is the most sensitive biochemical marker of bone resorption, however, given our results and the relative precision of crosslink measurement of $11 \%$ (Gallacher et al. Eur $\mathcal{F}$ Endocrinol 1994) and $0.8 \%$ for bone densitometry, the latter may be more useful in clinical practice over the long term.

\section{Markers of bone metabolism in asthmatic children receiving} inhaled corticosteroids

IOLO JM DOULL, M WHITE, NICHOLAS J FREEZER, STEPHEN T HOLGATE University Medicine, Centre Block, Southampton General Hospital, Southampton Osteocalcin is produced almost exclusively by osteoblasts, and as such is a sensitive index of bone formation. Collagen breakdown releases hydroxyproline, which is secreted in the urine, and is thus a measure of bone resorption. To determine the effect of inhaled cortiscosteroids on bone turnover in children we measured serum osteocalcin and urinary hydroxyproline in a group of 94 mildly asthmatic children receiving inhaled beclomethasone dipropionate (BDP) $200 \mu \mathrm{g}$ twice daily as a dry powder in a randomised, double blind, placebo controlled manner. Samples were collected prior to starting BDP and after three and six months treatment. Serum osteocalcin was measured by radioimmunoassay. An overnight urine was collected and urinary hydroxyproline measured as per Podenphant et al, and expressed as a hydroxyproline/creatinine ratio ( $\mathrm{Hyd} / \mathrm{Cr}$ ). There was no significant difference in serum osteocalcin at any of the three timepoints between the BDP and placebo treated children (baseline: $19.34 v 21.44 \mu \mathrm{g} / \mathrm{l}, \mathrm{p}=0.12$; three months: $18.34 v 20.20 \mu \mathrm{g} / \mathrm{l}, \mathrm{p}=0.12$; six months: $15.51 v 17.69 \mu \mathrm{g} / \mathrm{l}, \mathrm{p}=0.19$ ), or in $\mathrm{Hyd} / \mathrm{Cr}$ at any of the three timepoints (baseline: $17.8 v 19.5 \mu \mathrm{mol} /$ mmol, $\mathrm{p}=0.5$; three months: $14.5 \vee 16.1 \mu \mathrm{mol} / \mathrm{mmol}, \mathrm{p}=0.53$; $\mathrm{six}$ months: $13.2 v 18.2 \mu \mathrm{mol} / \mathrm{mmol}, \mathrm{p}=0.22$ ). Serum osteocalcin was significantly correlated at three $(r=0.5, \mathrm{p}=0.001)$ and six months $(r=0.53, \mathrm{p}=0.001)$ with growth over the six month period. At conventional doses BDP does not affect serum osteocalcin or urinary $\mathrm{Hyd} / \mathrm{Cr}$ in mildly asthmatic children.

\section{Suppressive effects of therapeutic doses of fluticasone pro-} pionate on the HPA axis in healthy volunteers

A GRAHNÉN, A LING-ANDERSSON, RM BRUNDIN, SÅ ECKERNÄS Pharmaco Medical Consultants, PMC AB, Uppsala, Sweden A new inhaled glucocorticosteroid, fluticasone propionate (FP), has recently become available. It has been claimed that FP has no clinically significant effects on the hypothalamic-pituitary-adrenal (HPA) axis, primarily based on data from single, morning plasma cortisol assessments. In two studies we have investigated the effect on the HPA axis following single and repeated doses of inhaled FP using a multiple sampling technique. Study 1: FP was administered as single doses (250, 500, and $1000 \mu \mathrm{g}$, respectively) and repeated doses (1000 $\mu \mathrm{g}$ bid) from Diskhaler. A single dose of budesonide (Bud) $(800 \mu \mathrm{g})$ from Turbuhaler was included as a reference. The study was of an open, partially randomised (single dose randomised), placebo controlled, crossover design. Twenty five healthy male volunteers took part in the study. For single doses of FP, the cortisol suppression, measured as $\mathrm{AUC}_{0-20 \mathrm{~h}}$, was $8 \%, 19 \%$ and $28 \%$ for $250 \mu \mathrm{g}, 500 \mu \mathrm{g}$ and $1000 \mu \mathrm{g}$. The Bud single dose resulted in a cortisol suppression of $16 \%$. Cortisol suppression, after all single doses, was significantly different from placebo. After seven repeated doses of fluticasone $(100 \mu \mathrm{g}$ bid), the cortisol suppression was $65 \%$. Study 2: FP from Diskhaler and Bud from Turbuhaler were given as single doses $(1000 \mu \mathrm{g}$ and $800 \mu \mathrm{g}$ respectively) and repeated doses $(1000 \mu \mathrm{g}$ bid and $800 \mu \mathrm{g}$ bid, respectively). The study was open, randomised, placebo controlled and crossover. Twenty four healthy male volunteers participated. In both studies the single doses were administered at $10.00 \mathrm{pm}$ and the repeated doses at $10.00 \mathrm{pm}$ and 10.00 am for 3.5 days. Multiple plasma cortisol samples were taken during a 20 hour period after each single dose and after the last (7th) repeated dose. Single doses of FP $(1000 \mu \mathrm{g})$ and Bud $(800 \mu \mathrm{g})$ resulted in a cortisol suppression of $25 \%$ and $26 \%$, respectively. Repeated dosing for 3.5 days resulted in a cortisol suppression of $55 \%$ for FP $(1000 \mu \mathrm{g}$ bid from Diskhaler $)$ and $34 \%$ for Bud $(800 \mu \mathrm{g}$ from Turbuhaler). This difference was statistically significant $(\mathrm{p}<0.001)$. We conclude that FP from Diskhaler, within the therapeutic dose range, results in a significant suppression of plasma cortisol which is more marked after repeated dosing. . . ज

\section{$\rightarrow$} $\omega$ $\overrightarrow{0}$ 윽 , . $\overrightarrow{0}$ ज

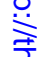
ปั㐅

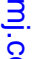

Genioglossus muscle response to negative airways pressure in awake humans: lack of effect of stimulus timing within the respiratory cycle

P NOLAN, DM O'LEARY, SA O'NEILl, SM ROE, RG O'REgAN, WT MCNICHOLAS Departments of Respiratory Medicine and Physiology and the Respiratory Sleep Laboratory, University College and St. Vincent's Hospital, Dublin, Ireland Upper airways (UA) negative pressure elicits a reflex increase in UA dilator muscle activity to help maintain a patent airway. Studies in anaesthetised rabbits have shown that the genioglossus (GG) response to UA negative pressure is greatest during early inspiration, protecting the UA at the time in the respiratory cycle when it is most vulnerable to collapse (Woodall et al. $\mathcal{F} A p p l$ Physiol 1989;67:366-70). The purpose of this study was to determine if, in awake humans, the GG response to negative airways pressure is influenced by the timing of the pressure stimulus within the respiratory cycle. We studied six healthy naive volunteers with their informed consent. The subjects lay supine and breathed through a nose mask. GG EMG was recorded using bipolar intraoral surface electrodes. Pulses of negative pressure $\left(350 \mathrm{~ms},-30 \mathrm{~cm} \mathrm{H}_{2} \mathrm{O}\right.$ or $0 \mathrm{~cm} \mathrm{H}_{2} \mathrm{O}$ ) were applied to the nose mask at eight times in the respiratory cycle, $\approx 100 \mathrm{~ms}, \approx 300 \mathrm{~ms}, \approx 600 \mathrm{~ms}$, and $\approx 1000 \mathrm{~ms}$ after the onset of inspiration (INSP) and $\approx 100 \mathrm{~ms}, \approx 300 \mathrm{~ms}, \approx 600 \mathrm{~ms}$, and $\approx 1000 \mathrm{~ms}$ after the onset of expiration (EXP). The GG EMG 
response was quantified as the natural logarithm (ln) of the ratio of the EMG activity during the pressure pulse to the EMG activity immediately preceding the pressure pulse; an $\ln$ ratio $>0$ implies increased activity. We found (figure) that pulses of $-30 \mathrm{~cm} \mathrm{H}_{2} \mathrm{O}$ airways pressure (O) significantly activated the GG muscle when compared with sham pulses of $0 \mathrm{~cm} \mathrm{H}_{2} \mathrm{O}(\mathrm{O})(\mathrm{p}=0.0004$, ANOVA with Student-Newman-Keuls test). However, there was no significant

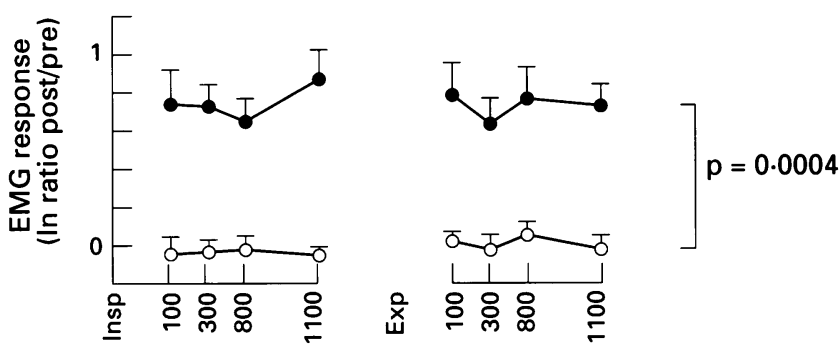

Time (ms)

difference between the responses to airways negative pressure applied at different times in the respiratory cycle. We conclude that, in contrast to findings in anaesthetised animals, the GG EMG response to airways negative pressure in awake humans is not influenced by the timing of the stimulus within the respiratory cycle.

\section{Reflex activity of palatal muscles in patients with sleep apnoea} and normal non-snoring subjects

IL MORTIMORE, NJ DOUglas Respiratory Medicine Unit, Department of Medicine, University of Edinburgh, Edinburgh In normal subjects genioglossus (Horner, $\mathcal{f}$ Physiol 1991;436:15), levator palatini and palatoglossus (Mortimore, Am f Respir Crit Care Med 1994;149:A145) exhibit reflex activity in response to negative pressure. This may be important in the maintenance of upper airways (UAW) patency, as collapse in sleep apnoea/hypopnoea (SAHS) patients is known to occur at either the retroglossal or retropalatal level. We have therefore compared the reflex responses of the antagonist palatal muscles levator palatini (LP) and palatoglossus (PG) during nose and mouth breathing to different levels $\left(0,-2 \cdot 5,-5,-7 \cdot 5,-10\right.$ and $\left.-12 \cdot 5 \mathrm{~cm} \mathrm{H}_{2} \mathrm{O}\right)$ of "square wave" negative pressure application in 13 non-snoring male subjects and 15 male SAHS patients using electromyography (EMG). Results were expressed as percentage maximum EMG activity. In normal subjects both LP $(p<0.001)$ and PG $(p<0.001)$ demonstrated increasing EMG activity in response to increasing negative pressure when applied via the nose or mouth (reaching $80 \%$ maximum at $-12.5 \mathrm{~cm} \mathrm{H}_{2} \mathrm{O}$ ). In SAHS patients LP demonstrated a significant increase in activity in response to negative pressure when applied via the nose $(p<0.006)$ and mouth $(p<0.04)$, reaching $43 \%$ and $55 \%$ respectively at $-12.5 \mathrm{~cm} \mathrm{H}_{2} \mathrm{O}$. PG showed only a non-significant trend to increasing activity with increasing negative pressure when applied via the nose $(p=0 \cdot 16)$ and mouth $(p=0 \cdot 19), 38 \%$ and $48 \%$ of maximum respectively at $-12.5 \mathrm{~cm} \mathrm{H}_{2} \mathrm{O}$. SAHS patients showed consistently lower responses to negative pressure application via both mouth and nose compared to normal subjects for both LP and PG $(p<0.001)$. We conclude that the reduced reflex response of LP and PG to negative pressure application in patients with SAHS may compromise UAW patency. [Supported by the Wellcome Trust.]

Comparison of reflex activity of levator palatini and palatopharyngeus in patients with sleep apnoea

IL MORTIMORE, NJ DOUGLAS Respiratory Medicine Unit, Department of Medicine, University of Edinburgh, Edinburgh Genioglossus (Horner, f Physiol 1991;436:15), levator palatini and palatoglossus (Mortimore, Am $\mathcal{F}$ Respir Crit Care Med 1994;149:A145) demonstrate reflex activity in response to negative pressure application, which may be important in maintenance of palatal position and upper airway patency. Furthermore, levator palatini (LP) and palatoglossus reflex activity in response to upper airway negative pressure application is decreased in sleep apnoea patients compared with normals. The respiratory activity of palatopharyngeus (PP) has not been investigated to date. We have therefore examined PP for evidence of respiratory and reflex activity in comparison with the antagonist muscle LP, using electromyography in seven patients with sleep apnoea. Results were expressed as \% maximum EMG activity in response to upper airway negative pressure application via nose or mouth. PP demonstrated phasic inspiratory activity during nose and mouth breathing. Application of negative pressure via the mouth caused a significant increase in $P P$ activity $(p=0.02)$ reaching $75 \%$ maximum at $-12.5 \mathrm{~cm} \mathrm{H}_{2} \mathrm{O}$ compared with $87 \%$ for LP $(p<0.001)$. Negative pressure applied via the nose caused only a non-significant trend to increased activity $(p=0.13)$ reaching $60 \%$ maximum at $-12.5 \mathrm{~cm} \mathrm{H}_{2} \mathrm{O}$ for PP compared with $80 \%$ for LP $(p<0.001)$. This differential response to nasal negative pressure application for $L P$ and PP may promote retropalatal obstruction in patients with sleep apnoea.

Effect of sleep fragmentation on daytime function

SE MARTIN, IJ DEARY, NJ DOUglas Respiratory Medicine Unit, Department of Medicine and Department of Psychology, University of Edinburgh, Edinburgh Patients suffering from the sleep apnoea/ hypopnoea syndrome (SAHS) show impaired daytime function and decreased sleep latencies on the MSLT. To help understand whether these features of SAHS are caused by sleep fragmentation or hypoxaemia, we have studied the effects of sleep fragmentation alone on daytime function in normal subjects. Nine normal subjects were studied over four nights and two days in the sleep laboratory. The nights were divided into two pairs. The first of each pair was for acclimatisation, and on the second the subject either slept undisturbed or had sleep fragmented with sound pulses every two minutes. Sound volume was titrated to cause a return to theta or alpha electrocorticol activity for a minimum of three seconds but no longer than 15 seconds. Both the second nights were followed by a full day's testing of daytime function using psychometric tests, the MSLT and the Maintenance of Wakefulness Test (MWT). Total sleep time did not differ between study nights (mean (SD) 400 (20) min undisturbed; 392 (19) min disturbed, $p=0 \cdot 5$ ). However, sleep architecture was altered during the fragmented night with significant increases in stages $1(p=0.02)$ and $2(p=0.002)$ and decreases in stage $4(p=0.001)$ and REM $(p=0.03)$. The time to REM onset was delayed on the fragmented night $(p=0 \cdot 04)$. Mean (SE) sleep latency decreased on MSLT (10 (1) $v 7$ (1) min, p<0.03) and MWT fell (29 (3) $v 20$ (3) $\min , \mathrm{p}<0.01)$ after fragmentation. Subjects showed a decrease in their levels of energetic arousal $(p=0.01)$ and an increased tense arousal $(p<0.05)$ on the UWIST scale after sleep fragmentation. One night of sleep fragmentation adversely affects the ability of normal subjects both to stay awake and to fall asleep. Energetic arousal is decreased and tense arousal is increased after a fragmented nights sleep. Their ability to perform other tasks of mental flexibility are not altered by sleep fragmentation in this ongoing study.

Evaluation of pulse transit time as a screening respiratory sleep study

DJ PITSON, JR STRADling Osler Chest Unit, Churchill Hospital, Oxford Two important components of a respiratory sleep study are a measure of inspiratory effort (marker of upper airway narrowing) and a measure of arousals (marker of sleep disturbance). In an all night beat-to-beat blood pressure (BP) profile, the size of the falls in systolic blood pressure (SBP) with inspiration (pulsus paradoxus) correlate well with the degree of inspiratory effort and there are rises in SBP with each arousal. Pulse transit time (PTT), measured from the ECG $R$ wave to detection of the pulse shock wave at the finger follows changes in BP well. The size of the inspiratory rises in PTT correlate well with inspiratory effort and falls in PTT are sensitive markers of arousal. Unlike SBP, PTT can be measured by a portable device. Twenty six patients presenting to the sleep unit for investigation of a possible sleep-related breathing disorder had a domiciliary PTT study and a laboratory PTT recording in conjunction with a polysomnography sleep study. PTT records wre analysed to calculate the mean size of the inspiratory rises in PTT for the night and the number of arousal-related falls in PTT per hour of the night. There were good correlations between the home and laboratory studies both for the mean size of the inspiratory rises in PTT $(r=0.93)$ and for the number of arousal-related falls in PTT/hour $(r=0.91)$. In the eight normal patients (no obstructive sleep apnoea (OSA) or snoring) the average inspiratory PTT rises ranged from 7 to $14 \mathrm{~ms}$ and in the nine patients subsequently established on nasal continuous positive airway pressure (NCPAP) for OSA PTT rises ranged from 14 to $39 \mathrm{~ms}$. 
PTT arousals ranged from 2 to 15 /hour in the normal subjects and from 15 to 94/hour in the NCPAP group. Thus, PTT data appear reproducible and capable of differentiating between two groups of patients defined as normal or requiring NCPAP on clinical history and polysomnography. PTT may therefore be a useful tool in a screening respiratory sleep study.

\section{Survey of pneumothorax management in Wessex}

E NEVILLE, J TURNER, D LIPSCOMBE, R LEA, J WADDELL and A ROBERTS ON BEHALF OF THE WESSEX THORACIC PHYSICIANS AND SURGEONS Chest Clinic, St Mary's Hospital, Portsmouth A survey of current clinical practice was conducted on 169 patients admitted in Wessex between 1 January 1992 and 1 July 1992 . Forty four (26\%) were managed by initial observation from one to 66 days, and only six patients had either aspiration or tube drainage at a later stage. Eighteen $(11 \%)$ were treated by initial aspiration. In nine of these a subsequent intercostal tube was inserted. One hundred and seven (63\%) patients were managed by initial intercostal tube drainage. Instructions about not clamping the tube were written in the notes in only $48(40 \%)$ of patients. Thirty four (30\%) patients had suction applied and, of those, only two were recorded as having a satisfactory apparatus applied. After the tube was removed, there was incomplete expansion of the lung in $28(29 \%)$ of cases. There were nine deaths, six with underlying respiratory disease, two of whom were under the age of 65 . Surgery was performed in $22(13 \%)$. Advice about return to work and lifestyle (for example, not flying) was recorded in only 36 (22\% of patients). We conclude that (1) the BTS guidelines do not adequately deal with removal of chest drains; (2) the management of chest drains and suction is unsatisfactory; and (3) advice to the patient at discharge was inadequate. We recommend that (1) each junior doctor's induction package should include four algorithms or lists: (a) management decisions, (b) management of chest drains, (c) when to refer for specialist respiratory advice, (d) discharge checklist; and (2) each ward where a chest drain may be cared for should have available a wall suction adaptor with clear instructions as to its use.

Audit of inpatient bronchoscopy requests: should hospital physicians have open access?

PH JOHNSON, H HOPKINSON, IDA JOHNSTON, WJM KINNEAR Queen's Medical Centre, University Hospital, Nottingham Open access to upper gastrointestinal endoscopy is commonplace, but it is traditional in most hospitals for a chest physician to assess referrals for bronchoscopy beforehand. This latter convention is time consuming. We have audited requests for bronchoscopy on inpatients to see if an open access service would be cost effective. Of 102 patients referred for a respiratory opinion over a period of six months, 18 involved a request for bronchoscopy. Nine were from medical specialties, seven from geriatrics, and two from surgical wards. All referrals were seen by a registrar or consultant in respiratory medicine. Of the 18 requests, 10 were considered to be appropriate by the respiratory physician who saw the patient, but four of these 10 were unfit for the procedure. In the other eight patients the referring clinicians had unrealistic expectations of the ability of bronchoscopy to yield the information that they required. Had an open access policy been in place in our hospital, 12 of the 18 patients referred would have had the procedure cancelled on arrival in the bronchoscopy room, entailing wasted bronchoscopy time and money, and distress to the patients. Even in those cases in whom bronchoscopy was subsequently performed, assessment of the patients beforehand by a respiratory physician was often valuable, for example in making provisional management plans. We conclude that all patients being considered for bronchoscopy should be seen by a respiratory physician, and that the time involved in these assessments is cost effective. Open access to bronchoscopy for hospital physicians might be appropriate if they were issued with guidelines on the indications and required fitness.

\section{Cigarette smoking and death certification}

TJ FLETCHER, RC HORTON, JG AYRES Chest Research Institute, Birmingham Heartlands Hospital, Birmingham; Cardiac Transplant Unit, Queen Elizabeth Hospital, Edgbaston Since 1992 doctors have been able to record cigarette smoking as a cause of death on death certificates without reporting the death to the coroner. In the 10 years prior to 1992 only some 200 such cases had been reported to the coroner countrywide despite an estimated 110000 deaths per annum caused by smoking related disease (BMF 1992;305:552). This new measure was taken in part to improve the accuracy of statistics on smoking-related deaths. We have gained the impression that, despite the new regulation, smoking is rarely recorded on death certificates. A retrospective analysis was undertaken of all deaths occurring in Birmingham Heartlands Hospital in January 1994. Age, sex and cause of death as recorded on the death certificate counterfoil was recorded in each case. One hundred and thirty two deaths were reported during this period ( 74 male, 78 female, mean age 75 , range 19-105). None had smoking recorded as a cause of death. Hospital notes were sought and details of smoking habit recorded. To date we have obtained 72 case notes for analysis. Of these, $32(44 \%)$ had inadequate documentation of smoking history in that we were unable to determine from the notes whether the patient had ever been a smoker. Five more $(7 \%)$ were comatose on arrival and unable to give smoking details, and nine $(13 \%)$ were non-smokers or had not smoked for at least five years. The remaining $26(36 \%)$ were still smokers at the time of hospital admission or had smoked within the last five years. From the causes of death recorded in this group 21 were considered to have died from a condition recognised to be smoking related (nine from bronchial carcinoma, five from chronic obstructive airways disease or emphysema, four from ischaemic heart disease, two from oesophageal carcinoma, and one from a stroke). We conclude that smoking is still not recorded on death certificates despite regulations designed to improve the accuracy of information regarding smokingrelated deaths.

Variability in the hospital management of acute emergencies and implications for future savings

H MOUdGIL, J HAMMERSLEY, M ANDERSON, AG LEITCH Respiratory Medical Unit, City Hospital, RIE NHS Trust It is assumed that the cost of care for a given patient is determined by the severity, complexity, or duration of the patient's illness with little attention paid to physician behaviour in this setting and despite variability in clinical practices. Four hundred and thirteen acute medical admissions to a specialist respiratory medical unit were audited to document the overall and diagnosis-related (DR) patterns of investigation (laboratory and radiological), duration of admission, and patient outcome (disposal) for the five consultant teams $(A=99, B=99, C=83, D=70, E=62)$ focusing on "routine" investigations and "repeated" investigations as the identified problem areas. Mean (SD) age for admissions was $64 \cdot 3$ (19.6) years (range 14-94) with $71 \cdot 1 \%$ due to respiratory disease with approximately $60 \%$ of the total due to either asthma, chronic bronchitis/emphysema $(\mathrm{CB} / \mathrm{E})$, or pneumonia. Of the initial investigations $46 \cdot 7 \% \mathrm{FBC}, 41 \cdot 6 \% \mathrm{U} \& \mathrm{E}, 34 \cdot 6 \% \mathrm{LFT}$ and $74.9 \% \mathrm{CXR}$ were abnormal; all groups subsequently repeated investigations more often where the initial outcome had been abnormal, indicating some discrimination in use. Investigations policy varied significantly between teams, especially in the category of "all diagnoses" with mean values for each investigation type for team A tending towards "zero" (haematology $p<0.01$, biochemistry $p<0.001$, virology $p<0.01$ ) and team E towards "infinity" (haematology $p<0 \cdot 05$, biochemistry $p<0.02$, bacteriology $p<0.001$, virology $p<0.01$, radiology $p<0.01)$. Management of DR groups was more standardised but continued to show a rationed use by team $A(p<0.01$ with biochemical tests for both $\mathrm{CB} / \mathrm{E}$ and pneumonia) without adversely affecting patient outcome and "over-investigation" by team $\mathrm{C}$ for pneumonia (haematology $\mathrm{p}<0.02$, biochemistry $\mathrm{p}<0.05$, virology $\mathrm{p}<0.02)$. Duration of stay was prolonged for team E $(p<0.05)$ for "all diagnoses" and for team $\mathrm{B}$ for pneumonia. Disposal also varied primarily between team A discharging more to the GP ( $p<0.01)$ with fewer follow ups $(p<0.02)$ and team $E$ discharging fewer to the GP $(p<0.001)$ with more follow ups $(\mathrm{p}<0.01)$; team $A$ had more GP and fewer follow ups (both $\mathrm{p}<0.05$ ) and team $\mathrm{E}$ the opposite (both $\mathrm{p}<0.01$ ) with $\mathrm{CB} / \mathrm{E}$, but practices were not significantly different for asthma or pneumonia. The audit concluded that most of the variability was in the nonrespiratory admissions but represented areas in which savings could be made. Objectives for testing differ and although it is not possible or even desirable to eliminate all unnecessary investigations the cost implications cannot be ignored. Investigations should be directed to where the result, even if normal, is important in the management of the patient. A change in physician behaviour is proposed which should include a regular assessment of "why" and "to what purpose" investigations are performed. 
Audit of discharge rates in respiratory outpatient clinics: NHS versus the private sector

JM WALES, E DRAPER The Glenfield Hospital NHS Trust and Department of Epidemiology and Public Health, University of Leicester, Groby Road, Leicester Outpatient referrals consume an increasing proportion of medical manpower. We have set out to evaluate the difference in follow up rates between private (consultant only) clinics and NHS clinics held during the same period. All new patients seen in one consultant's private practice in 18 months $(n=372)$ and two simultaneous NHS clinics $(n=527)$ were entered in the study. Demographic data, occupation and hence social class, together with diagnosis coded by ICD-9, were recorded. Follow up continued for a further 18 months. All private and all but five NHS patients were seen by one observer at the first consultation. Follow up was continued by the same observer in private practice and usually by registrars and senior house officers in the NHS sector. A marked difference in social group existed (social group I and II: NHS $n=109,20 \cdot 7 \%$; private sector $\mathrm{n}=178,47.8 \%, \mathrm{p}<0.0001)$. The table shows numbers of

\begin{tabular}{lllll}
\hline & 0 months & 6 months & 12 months & 18 months \\
\hline NHS & 150 & 126 & 65 & 54 \\
\multirow{2}{*}{ Private } & $(28 \cdot 5 \%)$ & $(23 \cdot 9 \%)$ & $(12 \cdot 3 \%)$ & $(10 \cdot 2 \%)$ \\
& 27 & 18 & 11 & 9 \\
& $(7 \cdot 3 \%)$ & $(4 \cdot 8 \%)$ & $(3 \cdot 0 \%)$ & $(2 \cdot 4 \%)$ \\
\hline
\end{tabular}

patients attending at six monthly intervals after entry into the study was closed: 317 private (85.4\%) and 340 NHS (64.5\%) were discharged by the second follow up appointment. There was an excess in the NHS sector of patients with chronic airways limitation and lung cancer. Asthma was heavily represented in both groups. The reasons for such a marked variation in follow up rates are discussed. We feel that the study supports the value of consultant only clinics in the NHS to support patients who may be more socially disadvantaged or sicker than those referred privately.

\section{Characteristics of infants ventilated with fast rates who receive} muscle relaxants

DP COCHRAN, NJ SHAW Neonatal Unit, Liverpool Maternity Hospital, Liverpool With the increasing use of fast ventilator rates (60-80 breaths $/ \mathrm{min}$ ) fewer preterm infants appear to require muscle relaxants. However, even with faster rates there remain a group of babies for whom they are prescribed on subjective, clinical criteria. We studied 97 infants (birth weight $<2 \mathrm{~kg}$ ), ventilated using rapid rates, of whom $24(24 \cdot 7 \%)$ received muscle relaxants as judged necessary by the attending clinicians. There was no significant difference in birth weight or gestation between the two groups. Infants who were paralysed had markedly more severe lung disease with higher inspired oxygen concentrations (median $1.0 v 0.65, \mathrm{p}<0.0001$ ) and peak inspiratory pressures (median $27.5 v 22.0, \mathrm{p}<0.0001$ ) on day 1 . The paralysed group suffered high mortality $(10 / 24 v 8 / 73, \mathrm{p}<0.001)$, a higher incidence of parenchymal cerebral haemorrhage $(6 / 25 v 4 / 72, \mathrm{p}<0.05)$, required inotrope more frequently, $(13 / 23 v 19 / 73, \mathrm{p}<0.05)$ and had a higher incidence of pneumothorax $(6 / 25 v 10 / 72$, NS). The risk of death or chronic lung disease was higher in the paralysed group but was only significant at 36 weeks gestation $(17 / 25 v 28 / 72, \mathrm{p}<0.05)$ and not at 28 days of age. These data suggest that infants who receive muscle relaxants are likely to have very severe lung disease and ultimately a poor prognosis. There are a number of possible explanations for these associations. We believe these data should prompt a re-examination of the use of muscle relaxants in the era of fast rate ventilation to ensure that their prescription is appropriate and that they are not contributing to the poor outcome in this group of babies. Newer strategies of ventilation may hold more promise for these patients.

Value of flexible fibreoptic bronchoscopy in children with persisting lobar collapse

DE LACY, RL SMYTH, DP HEAF Respiratory Unit, Royal Liverpool Children's NHS Trust, Alder Hey, Liverpool Flexible fibreoptic bronchoscopy has been available at Alder Hey Hospital since 1992. We have reviewed the results of bronchoscopy in children who presented with persisting lobar collapse (despite conventional treatment of two weeks antibiotics and physiotherapy) from January 1992 to April 1994. Twenty two children ( 7 girls, 15 boys) with a mean age of 6.02 years (range $0.83-12.08$ ) were investigated by bronchoscopy for persisting lobar collapse. Ten patients had left lower lobe collapse, nine right middle lobe collapse, five right lower lobe collapse, two right upper lobe collapse, one lingula lobe collapse, and one left lung collapse. Findings at bronchoscopy were as follows: collapse/no infection $=6$, collapse/persisting infection $=4$, obstruction of bronchi $=7$ (three were obstructed by peanuts previously unsuspected, two by mucous plugs), normal $=5$. Outcome if bronchoscopy abnormal: resolution of collapse $=6$, collapse improved $=2$, persistent collapse with evidence of bronchiectasis $=4$, lobectomy $=2$. Persisting lobar collapse in children should be managed actively to prevent the development of permanent collapse and bronchiectasis. Flexible fibreoptic bronchoscopy is useful in the management of persisting lobar collapse as it can detect causes of bronchial obstruction (including an unsuspected foreign body) and allows suction to clear mucous plugs. We suggest that any child who has lobar collapse that persists after conventional treatment should have a bronchoscopy.

Short term respiratory outcome in infants with chronic lung disease of prematurity (CLD): risk of admission/sudden infant death syndrome (SIDS)

R ILES, AT EDMUNDS The Royal Hospital for Sick Children, Edinburgh Thirty five infants born between 24 and 31 weeks gestation (mean (SD) 27 (1.9) weeks) weighing a mean (SD) of 1010 (71)g at birth were studied between the 39th and 44th week of post-conceptional age. All infants required ventilatory support from birth (mean (SD) $18.7(3.11)$ days). Thirty three infants had a chronic oxygen requirement at 36 weeks. Infants were studied using the squeeze technique to measure VmaxFRC and overnight assessment of oxygen saturation $\left(\mathrm{SaO}_{2}\right)$. Analysis of overnight saturation excluded movement artefact. At time of examination eight infants were on supplementary oxygen $\left(\mathrm{SaO}_{2}\right.$ was assessed in air for short periods only), no child was on theophylline treatment. The clinical course of the cohort was recorded over the next three months. Nineteen infants were readmitted to hospital with respiratory related illness $(76 \%)$, twelve with proven respiratory syncytial virus (RSV) infection, eight with a history suggestive of an acute life threatening event (ALTE). One child died unexpectedly, the post-mortem examination was highly suggestive of SIDS showing minimal changes of CLD. For all infants mean $\mathrm{SaO}_{2}$ correlated with risk of admission $(\mathrm{t}=4.47, \mathrm{p}<0.001)$ and ALTE/SIDS $(t=2 \cdot 8, p<0 \cdot 001)$, the variability of the $\mathrm{SaO}_{2}$ correlated with risk of admission $(t=2, p<0.05)$ and ALTE/SIDS $(t=3.6$, $\mathrm{p}<0.001)$. For the non-oxygen dependent infants mean $\mathrm{SaO}_{2}$ correlatd with ALTE/SIDS $(t=2 \cdot 1, p<0.05)$ and the variability of the $\mathrm{SaO}_{2}$ correlated with ALTE/SIDS $(t=3 \cdot 38, p<0 \cdot 005)$. Those infants who were readmitted following RSV infection were graded according to their clinical severity. For this group the mean $\mathrm{SaO}_{2}$ highly correlated with the severity of illness on admission $(R=0.77, \mathrm{p}<0.005)$. VmaxFRC did not correlate with clinical outcome.

\section{Influence of maturity at birth on lung volume of preschool} children

F GRIFFIN, A GREENOUGH Paediatric Respiratory Laboratory, Departments of Child Health and Thoracic Medicine, King's College Hospital, London In a prospective follow up study of prematurely born children we demonstrated that, at approximately four years of age, $41 \%$ were hyperinflated - that is, they had a functional residual capacity (FRC) greater than $120 \%$ of that predicted for their height. No control group, however, was included, thus it was not possible to determine whether the hyperinflation related to the high proportion of children who were symptomatic or to premature birth per se. We have now therefore matched for height 46 of the prematurely born children (study group) with children born at term who had no respiratory problems (controls). In both groups of children FRC was measured by helium gas dilution. The study group was born at a median gestational age of 29 weeks (range 24-35), 27 had been ventilated in the neonatal period and 15 were symptomatic at four years. The median FRC of the study group $(670 \mathrm{ml}$, range $420-1260)$ was significantly higher than that of the control group (641 ml, 370-950), $\mathrm{p}<0 \cdot 01$. No such difference, however, was seen when the asymptomatic prematurely born children were compared with their matched 
controls ( $685 \mathrm{ml}, 420-1260 v 711 \mathrm{ml}, 400-950)$. Fifteen of the study group were hyperinflated. The hyperinflated children in the study group did not differ significantly from the rest of their cohort regarding their gestational age (median 29 weeks, 25-35 $v 29$ weeks, 24-32). A greater proportion of the hyperinflated children, however, were symptomatic $(67 \% v 19 \%) \mathrm{p}<0.01$. We conclude hyperinflation in prematurely born children of preschool age does not appear to be influenced by maturity at birth.

\section{Maternal asthma, premature birth and the risk of respiratory morbidity in primary schoolchildren}

YJ KELLY, BJ BRABIN, P MILligaN, D HEAF, J REID, MG PEARSON School of Tropical Medicine, Alder Hey, Sefton Health and Aintree Chest Centre, Liverpool Maternal asthma and prematurity are known to predispose to the development of later childhood respiratory morbidity. We analysed the relationship of maternal asthma, premature birth and later respiratory outcome in data from parent completed questionnaires collected during two cross sectional community surveys of primary school children, aged 5-11 years, in Merseyside during 1991 and 1993. The numbers of children surveyed were 1872 and 3746 respectively. The proportions of preterm deliveries among asthmatic mothers in the surveys were $23 \cdot 7 \%(28 / 118)$ in 1991 and $19 \cdot 1 \%(61 /$ 319 ) in 1993 , compared with $14.0 \%$ and $13.0 \%$ of non-asthmatic mothers delivering prematurely. Asthmatic mothers are more likely to have a preterm delivery than non-asthmatic mothers (OR 1.49, $95 \%$ CI $1 \cdot 10$ to 2.02 ). Smoking is a separate risk factor for preterm delivery (OR $1 \cdot 35,95 \%$ CI $1 \cdot 10$ to $1 \cdot 65$ ). Asthmatic mothers did not have an increased risk of delivering small, growth retarded babies. Maternal asthma, paternal asthma and premature birth, in that order, increased the risk of later childhood respiratory morbidity (OR 3.13, $95 \% \mathrm{CI}, 2 \cdot 36$ to $4 \cdot 16 ; 2 \cdot 23,1 \cdot 62$ to $3 \cdot 05 ; 1 \cdot 40,1 \cdot 10$ to $1 \cdot 79)$. Babies born small but full term are less likely to develop respiratory symptoms in childhood, although this is not statistically significant (OR 0.63 , $95 \%$ CI $0 \cdot 28$ to $1 \cdot 41$ ). The effect of maternal asthma on preterm delivery is independent to that of smoking during pregnancy. Maternal asthma is associated with an increased number of premature (but normal size for dates) babies. Prematurity has a lesser effect than genetic factors on predisposing to the later development of respiratory symptoms.

\section{Association of family size with atopic disease}

D JARVIS, P BURNEY, $S$ CHINN, C LUCZYNSKA, E LAI, R HALL, B HARRISON, J STARK Department of Public Health Medicine, UMDS; Department of Respiratory Medicine, The Ipswich Hospital; Department of Respiratory Medicine, West Norwich Hospital; Department of Respiratory Medicine, Addenbrookes Hospital, Cambridge It has been postulated that atopic disease is inversely associated with family size (Strachan BMF 1989; 299:1259-60). Data collected as part of the British arm of The European Community Respiratory Health Survey were analysed to determine the association of family size with symptoms suggestive of current asthma and hayfever in a random sample of 1094 young adults living in East Anglia. Of those interviewed $866(79 \cdot 1 \%)$ agreed to have blood tests for determination of specific IgE to house dust mite (HDM) and timothy grass (Pharmacia Diagnostics). The prevalence of symptoms/sensitisation by number of siblings is shown in the table.

\begin{tabular}{|c|c|c|c|c|c|}
\hline & \multicolumn{5}{|c|}{ Number of sibs } \\
\hline & 0 & 1 & 2 & 3 & $4+$ \\
\hline Wheeze & 31.9 & $27 \cdot 8$ & $25 \cdot 8$ & $32 \cdot 1$ & $24 \cdot 5$ \\
\hline Woken by SOB & $5 \cdot 3$ & $11 \cdot 4$ & $7 \cdot 8$ & $10 \cdot 2$ & $7 \cdot 0$ \\
\hline Asthma attack & $4 \cdot 2$ & $7 \cdot 0$ & $6 \cdot 0$ & 3.8 & $2 \cdot 3$ \\
\hline Hayfever** $+\dagger$ & $33 \cdot 0$ & $34 \cdot 3$ & $27 \cdot 8$ & $28 \cdot 2$ & $22 \cdot 2$ \\
\hline \multicolumn{6}{|l|}{ Specific IgE to } \\
\hline HDM & $31 \cdot 0$ & $26 \cdot 2$ & $22 \cdot 4$ & $25 \cdot 0$ & $22 \cdot 4$ \\
\hline Grass* & $25 \cdot 4$ & $30 \cdot 1$ & $23 \cdot 2$ & $21 \cdot 8$ & 20.9 \\
\hline
\end{tabular}

${ }^{*} \mathrm{p}<0.05, * * \mathrm{p}<0.01\left(\chi^{2}\right.$ for trend significance).

$+\mathrm{p}<0.05,+t \mathrm{p}<0.01$ ( $\chi^{2}$ for trend significance after adjustment for age, sex smoking and area).

Although there was an association of family size with some of the symptoms, birth order was associated with hayfever only. The prevalence of reported hayfever and sensitisation to grass were negatively associated with family size. The relationship of family size to asthma and sensitisation to house dust mite was less clear. [This work was supported by the National Asthma Campaign.]

Synthesis of IL-8, GM-CSF and TNF $\alpha$ by cultured nasal epithelial cells from atopic rhinitic, atopic non-rhinitic and non-atopic non-rhinitic subjects and the effect of exposure to nitrogen dioxide $\left(\mathrm{NO}_{2}\right)$

MA CALDERON, JL DEVALIA, RJ SAPSFORD, A PRIOR, RJ DAVIES Departments of Repiratory Medicine and Allergy, ENT, St Bartholomew's Hospital, London Recent studies have suggested that airway epithelial cells of atopic and non-atopic individuals may differ in their ability to generate inflammatory mediators. We have cultured nasal epithelial cells (NEC) from biopsy tissues of 10 atopic rhinitic subjects (AR), nine atopic non-rhinitic subjects (AnR), and eight non-atopic non-rhinitic subjects (normals) and investigated the ability of these cells to release GMCSF, IL-8 and TNF $\alpha$ and the effect of release of these mediators after exposure for six hours to $400 \mathrm{ppb} \mathrm{NO}_{2}$. Cells were grown as confluent explant cultures on tissue culture inserts and the cytokines released into the medium were estimated by ELISA. NEC from AR released significantly greater $(p<0.05)$ amounts of IL-8, TNF $\alpha$, and GM-CSF than NEC from AnR and normals under normal culture conditions. IL- 8 was generated in greatest quantity and GM-CSF in lowest quantity, irrespective of whether the cells were derived from atopic or non-atopic subjects. Exposure of NEC for six hours to $400 \mathrm{ppb} \mathrm{NO}$ led to significant release of IL-8 in the normal and the AR group (14.2 and $62 \mathrm{pg} \mathrm{IL}-8 / \mu \mathrm{g}$ cellular protein, respectively) compared with NEC exposed to air $(3.26$ and $44 \mathrm{pg} / \mu \mathrm{g}$ cellular protein, $\mathrm{p}<0.05$, respectively). Release of $\mathrm{TNF} \alpha$ was significantly increased from $0.7 \mathrm{pg} / \mu \mathrm{g}$ cellular protein to $1.22 \mathrm{pg} / \mu \mathrm{g}$ cellular protein $(p<0.05)$, only in NEC from the normal group after exposure to $\mathrm{NO}_{2}$. These results suggest that NEC from atopic rhinitic individuals release more GM-CSF, IL-8 and TNF $\alpha$ than NEC from atopic non-rhinitic and normal volunteers and that exposure to $\mathrm{NO}_{2}$ may influence the synthesis of pro-inflammatory cytokines primarily from the NEC of non-atopic non-rhinitic individuals.

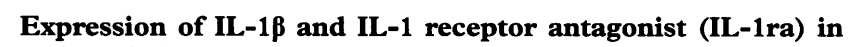
asthmatic bronchial epithelium: effect of steroids

AR SOUSA, SJ LANE, JA NAKHosteen, RN POSTON, TH LEE Departments of Allergy and Respiratory Medicine and Experimental Patholoy, UMDS, Guy's Hospital, London; Department of Respiratory Medicine, Augusta Teaching Hospital, Bochum, Germany Accumulating evidence suggests that the cytokine network is central to the immunopathology of bronchial asthma. Recent evidence for the existence of naturally occurring cytokine antagonists has added to this complexity. In this study we looked at the expression of IL-1 $\beta$ and its naturally occurring receptor antagonist, IL-1 ra, in normal and asthmatic bronchial epithelium and have investigated the effect of inhaled beclomethasone dipropionate. Frozen bronchial biopsies from 12 normal and 18 asthmatic individuals were stained with a rabbit anti-IL-1 $\beta$ (R\&D) and a rabbit anti-IL-1 ra (Prof WP Arend, Colorado). Hue-saturationintensity colour image analyses (HSI) were used to quantify the brown immunoperoxidase reaction colour present on the bronchial epithelium. There was an increased expression of both IL-1 $\beta$ and IL$1 \mathrm{ra}$ in the asthmatic bronchial epithelium, $\mathrm{p}<0.0001$ for both. There was a significant correlation between the percentage epithelium stained for IL- $\beta$ and for IL-ra $(r=0.65, p<0.001)$. Additionally, frozen biopsies from six asthmatic individuals on $1000 \mu \mathrm{g}$ beclomethasone per day for eight weeks and from six asthmatic individuals on matching placebo were stained with the above anti-IL-1 $\beta$ and anti IL-1 ra antibodies and the immunohistochemical results were quantified by HSI. There was a significant decrease in the percentage change in epithelial expression of IL-1 $\beta$ by beclomethasone $(p>0.01)$ but not of IL-1 ra $(\mathrm{p}<0 \cdot 3)$. Therefore, IL-1 $\beta$ and IL-1 ra are expressed more in the epithelium of asthmatic airways and becolmethasone selectively inhibits IL-1 $\beta$ epithelial expression without affecting IL-1 ra significantly.

Increased $\mathrm{H}_{2} \mathrm{O}_{2}$ generation by primed human eosinophils following allergen challenge

DJ EVANS, MA LINDSAY, BJ O'CONNOR, PJ BARNES Department of Thoracic Medicine, National Heart and Lung Institute, London; Clinical Studies 
Unit, Royal Brompton Hospital, London Activation of inflammatory cells is known to play a part in the pathological changes seen in the asthmatic airway. In this study we have examined the possibility that the eosinophil is activated in asthma using allergen challenge as a model of allergic inflammation. The effect of allergen challenge on the ability of ex vivo human eosinophils to release hydrogen peroxide following stimulation with platelet activating factor (PAF) and complement factor 5a (C5a) was studied in five mild asthmatics. Subjects were venesected immediately before and 24 hours after the allergen challenge. All subjects had a late asthmatic response with a mean maximum fall in $\mathrm{FEV}_{1}$ of $29 \%$ between 4 and 10 hours after challenge. Freshly separated eosinophils ( $>95 \%$ purity) were incubated in fibrinogen coated wells $(100 \mu \mathrm{g} / \mathrm{ml})$ for one hour. To each well there were serially increasing doses of PAF of C5a. Hydrogen peroxide levels were measured in the supernatant of the cell culture following this incubation. The dose response curves and time course plots showed a dose and time dependent effect for both PAF and C5a. The maximum $\mathrm{H}_{2} \mathrm{O}_{2}$ release plateaued at one hour. The maximum response for PAF was achieved at a dose of $10^{-6} \mathrm{M}$ and for C5a, $10^{-7} \mathrm{M}$. Allergen challenge resulted in a priming effect on the eosinophils as measured by changes of $\mathrm{H}_{2} \mathrm{O}_{2}$ released by the cells. This reached a level of significance for PAF $(p<0.02)$, but not for C5a $(p=0 \cdot 2)$. Additionally adhered cells in the absence of stimulation had increased capacity to release $\mathrm{H}_{2} \mathrm{O}_{2}$ after allergen challenge. These results demonstrate an in vivo priming of human eosinophils following allergen challenge. This augmented cellular response is likely to be an important factor in the pathogenesis of the late asthmatic response.

\section{Human bronchial epithelial cells express and release RANTES}

JL DEVALIA, JH WANG, RJ SAPSFORD, RJ DAVIES Department of Respiratory Medicine and Allergy, St Bartholomew's Hospital, London Although several cell types, including $\mathrm{T}$ lymphocytes, fibroblasts, endothelial cells and renal tubular epithelial cells, are known to express the chemokine RANTES, to date there is little information on the expression of this cytokine by human bronchial epithelial cells. We cultured human bronchial epithelial cells (HBEC) from surgical tissue in vitro and investigated these for their ability to synthesise and release RANTES, both constitutively and after stimulation with TNF $\alpha$. Additionally, we have obtained bronchial biopsies from mild asthmatic patients, treated with either inhaled beclomethasone dipropionate (BDP) $500 \mu \mathrm{g} \mathrm{bd}$ or matched placebo for four months and investigated these for the presence of RANTES in the bronchial epithelium, before and after treatment. RANTES in the biopsies was detected by immunocytochemistry and expressed as the percentage of total epithelium staining for RANTES, by use of a colour image analysis system. Measurement of RANTES released from HBEC demonstrated that this cytokine was synthesised constitutively and that this was significantly increased by TNF $\alpha$ in a dose-dependent manner (range $10-63 \mathrm{fg} / \mu \mathrm{g}$ cellular protein) after 24 hours. Treatment with anti-TNF $\alpha$ blocked the TNF $\alpha$-induced release of RANTES from the HBEC. Staining of biopsies demonstrated that RANTES was expressed in the bronchial epithelium of mild asthmatics in vivo and that treatment with BDP led to a significant decrease in the expression of RANTES from $17 \cdot 12 \%$ to $4 \cdot 22 \%(p<0 \cdot 05)$. These results suggest that human bronchial epithelial cells are capable of synthesising RANTES, both in vitro and in vivo, and may play a part in the pathogenesis of asthma. Furthermore, corticosteroid treatment may influence airway inflammation by modulating the expression and/or release of proinflammatory cytokines in the bronchial epithelium.

\section{Mediators from human bronchial epithelial cells can influence eosinophil and neutrophil chemotaxis in vitro}

MM ABDELAZIZ, JL DEVALIA, OA KHAIR, RJ SAPSFORD, RJ DAvies Department of Respiratory Medicine and Allergy, St Bartholomew's Hospital, London We have recently demonstrated that human bronchial epithelial cells are capable of expressing and releasing proinflammatory cytokines including GM-CSF, IL-8 and TNF $\alpha$ (Devalia et al. Am ₹ Respir Cell Mol Biol 1993;9:271-9). To investigate the biological relevance of the release of these cytokines we have collected 24 hours conditioned medium (CM) from confluent explant cultures of human bronchial epithelial cells, and investigated the effect of this medium on the chemotactic response of preparations of eosinophils and neutrophils isolated from normal individuals. Chemotaxis was assessed according to the Boyden technique and results were expressed as the mean number of cells in 10 random high power fields. CM significantly increased eosinophil chemotaxis (median $11 \cdot 4$, range $9 \cdot 1-12 \cdot 3 ; p=0 \cdot 005$ ) compared with medium 199 (negative control, median $4 \cdot 1$, range $3 \cdot 4-7 \cdot 0$ ), but not when compared with $10 \mu \mathrm{m}$ PAF (positive control, median $20 \cdot 0$, range $13 \cdot 0-26 \cdot 5$ ). Similarly, CM also significantly increased neutrophil chemotaxis (median $30 \cdot 4$, range 19.4-33.3; $>>0.01$ ) compared with medium 199 (median $17 \cdot 2$, range $13 \cdot 4-25 \cdot 3$ ), but not when compared with $10^{-9} \mathrm{M}$ f-MetLeu-Phe (positive control, median 64.7, range 49.0-132.0). These results suggest that human bronchial epithelial cells produce inflammatory mediators which can influence the activity of eosinophils and neutrophils and consequently may play an important part in the aetiology of airways disease.

Accident and emergency attendance for asthma: an opportunity for intervention?

PJM VICKERY, BJ MCDONOUGH, DPS SPENCE, CRK HIND Royal Liverpool University Hospital Trust (RLUHT), Liverpool The attendance of an asthmatic patient at an Accident and Emergency (A\&E) department is a failure of treatment. Patients attending $A \& E$ may be either admitted to the hospital under the care of physicians, admitted to the short stay observation ward (SSOW) in A\&E, or discharged. The care of those admitted to hospital under physicians has been audited nationally (Pearson et al. Thorax 1992). However, patients whose care has been A\&E based have not been so well examined. Since October 1993 an asthma liaison nurse (ALN) has been attached to the A\&E department of the RLUHT and is responsible for assessing patients in the SSOW and advising appropriately. During the six months (January-June 1994) 344 patients attended $A \& E$ with a diagnosis of asthma: 141 were admitted to a hospital bed [median age 53 years (range 17-91)], 126 were admitted to SSOW [median age 33 years (range 17-87)], and 77 [median age 30 years (range 13-71] were discharged. A representative cohort of 44 patients [median age 31 years (range 18-71)] admitted to the SSOW were assessed by the ALN (two patients attended twice: 46 episodes). The duration of increasing asthma symptoms prior to admission was $<24$ hours in 8 patients, 24-72 hours in six patients, and $>72$ hours in 32 patients. Only $43 \%$ had sought help for their asthma since increasing symptoms had begun. Despite the fact that $72 \%$ of the GP practices of these patients were approved for asthma chronic disease management programmes by the Family Health Services Authority, only $25 \%$ had attended for asthma review in the previous year. $64 \%$ of patients possessed a peak flow meter but only one was using it, and $75 \%$ had been prescribed inhaled steroids. Modifiable factors in their asthma management were identified by the ALN in all cases: education (43), inhaler technique (29), poor compliance with prophylaxis (17), a need to increase prophylaxis (32), need for a peak flow meter prescription (16), and a self management plan (36). We conclude that asthma patients attending $A \& E$ are frequently reluctant to seek help from their GP and, as a result, their care is often suboptimal. Attendance at $A \& E$ offers a unique opportunity to advise these patients on their asthma management, hopefully reducing morbidity and future hospital attendance.

\section{Risk of severe life threatening asthma (SLTA) and death and type of prescribed $\beta$ agonist: an example of confounding by severity}

JE GARRETT, S LANES, J KOLBE, HH REA Respiratory Services, Green Lane Hospital, Auckland, New Zealand; New England Epidemiology Institute, Newton Lower Falls, Massachusetts, USA In previous case-control studies examining the association between inhaled $\beta$ agonists in death or near death, the potential for confounding by disease severity has precluded confirmation of a causal association between asthma drugs and death or SLTA. The aims of this study were (1) to assess the relationship between inhaled $\beta$ agonists (specifically fenoterol) and other asthma drugs and the risk of asthma death or SLTA, and (2) to examine the influence of confounding by severity by controlling for a variety of risk factors not reliably recorded in previous studies. A retrospective, dynamic cohort study was performed on patients aged 13-52 years following a hospital visit (ER visit or admission) between May 1986 and December 1987. Following the "index event" each subject contributed person time to the analysis until death or April 1989 , allowing incidence rates for SLTA or death to be calculated. The rates of each outcome in relationship to each drug were calculated, as were incident rate ratios (RRs) for users to non-users. For each type of drug adjusted RRs were calculated, controlling for four risk 
factors which were independently associated with an increased risk of SLTA (race, previous hospital admissions, usual asthma symptoms, and severity of previous attack). Six hundred and twenty four patients were recruited subsequent to which there were 14 asthma deaths, 94 SLTAs, 333 hospital admissions, and 520 ER visits. Crude RRs for SLTAs and death were strikingly similar and were increased for all asthma drugs, except for inhaled $\beta$ agonists. After controlling for the independent risk factors, most of the crude RRs reduced to a nonsignificant association. Controlling for hospitalisation in the past year and any oral corticosteroid use reduced the RR for inhaled fenoterol when compared with inhaled salbutamol from 2.02 to 1.52 (CI 1.02 to $2 \cdot 28$ ). When the four independent risk factors were controlled for, the $\mathrm{RR}$ for fenoterol reduced further to $1 \cdot 11$ (CI 0.57 to $2 \cdot 20$ ). These data support the notion that most asthma medications are related to both asthma severity and risk of SLTA (and death) and such associations result in substantial confounding by severity. Users of fenoterol had a higher incidence of life-threatening asthma than users of salbutamol. However, after adjusting for differences in baseline severity, users of fenoterol and of salbutamol had similar risks of life threatening asthma. [Supported by the Asthma Foundation of New Zealand.]

\section{Beta agonist type and outcome following severe life threatening} attack of asthma (SLTA)

J KOLBE, S LANES, JE GARRETT, H REA Respiratory Services, Green Lane Hospital, Auckland; New England Epidemiology Institute, Newton Lower Falls, Massachusetts, USA Confounding by severity remains a tenable explanation for the reported association between prescription of the $\beta$ agonist fenoterol and asthma death or SLTA. Previous SLTA is associated with a markedly increased risk of asthma death and further SLTA. The aims of this study were (1) to assess the relationship between inhaled $\beta$ agonists, specifically fenoterol, and the risk of asthma death or SLTA, in a group of subjects at recognised high risk, and (2) to examine the influence of confounding by severity by controlling for a variety of risk factors. A retrospective dynamic cohort study was performed on patients aged 15-50 years following an

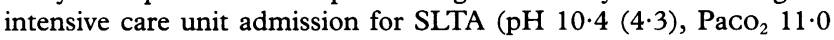
$(5 \cdot 5) \mathrm{kPa}$ on admission). Each subject contributed person time to the analysis until death or April 1989, allowing incidence rates for SLTA or death to be calculated. The rates of each outcome were calculated as were the incident rate ratios (RRs) for fenoterol to salbutamol users. Adjusted RRs were calculated by controlling for risk factors associated with an increased risk of SLTA in non-fenoterol users. Three hundred and seventy eight patients formed the cohort. There were 13 deaths, 62 subsequent SLTAs and 345 subsequent hospital admissions. Prescription of fenoterol was associated with an increased risk of SLTA (crude RR $=1 \cdot 72$ ). Controlling for socioeconomic status was associated with an adjusted RR of 1.44 (95\% CI 1.05 to 1.97 ). After additional adjustment for multiple risk factors (age, prior SLTA, number of types of inhaled $\beta$ agonist prescribed), the adjusted RR was 1.02 ( $95 \%$ CI 0.57 to 1.83 ). We conclude that, in this high risk group, the increased risk of SLTA associated with fenoterol prescription seems best explained by confounding. [Supported by the Asthma Foundation of NZ and the NZ Lotteries Board.]

Comparison of nebulisation therapy combining ipratropium bromide $(0.5 \mathrm{mg})$ plus salbutamol sulphate $(3.0 \mathrm{mg})$ with salbutamol sulphate $(3.0 \mathrm{mg})$ alone in acute asthma

JE GARRETT, I TOWN for the New Zealand Combivent Study Group Respiratory Services, Green Lane Hospital, Auckland, New Zealand; The Canterbury Research Group, Christchurch, New Zealand There is uncertainty about the benefit of routine addition of ipratropium bromide (IB) to $\beta$ agonist therapy in acute asthma. Patients with more severe acute asthma appear to benefit more from combination therapy. We aimed to evaluate (1) whether a combination of nebulised IB $(0.5 \mathrm{mg})$ plus salbutamol sulphate (SS) $(3.0 \mathrm{mg})$ confers significant additional bronchodilating effects over nebulised SS (3.0 mg) in patients with acute bronchial asthma, and (2) whether adjusting for the effects of known prognostic indicators of outcome (baseline $\mathrm{FEV}_{1}$, age, duration of asthma attack and medicines taken in the six hours prior to presentation) influences any of the additional effect seen with IB. A double blind, two centre, randomised, parallel group, single dose study of 338 asthmatics aged 18-55 years attending an emergency department (ED) with acute asthma. The primary end point was $\mathrm{FEV}_{1}$ measured at 45 and 90 minutes. The mean (SE) absolute difference in $\mathrm{FEV}_{1}$ with IB plus SS compared with SS at 45 minutes was $93(44) \mathrm{ml}(\mathrm{p}<0.05)$ and at 90 minutes $113(48) \mathrm{ml}$ $(p<0 \cdot 05)$. Treatment response was predicted by baseline $\mathrm{FEV}_{1}$ $(p<0.001)$, time between onset of asthma attack and start of nebulisation $(p<0.05)$, age $(p<0.05)$, and inhaled $\beta$ agonist $(p<0.0001)$ and inhaled anticholinergics $(p<0.01)$ used in the previous six hours. The added advantage of combination therapy response over $\beta$ agonist response was apparent in all subgroups defined by these prognostic factors. Those subjects who had taken no asthma medication in the six hours prior to ED attendance exhibited the greatest response to combination therapy. Thus, there was a significantly greater response $\vec{A}$ at both 45 and 90 minutes with the combination therapy. Patients with more severe asthma at presentation did not exhibit greater benefit $\bar{\sigma}$ from the addition of IB. Patients who exhibited most benefit from $\bar{G}$ IB were those who had consumed least bronchodilator prior to presentation and who were presumably on the lower part of the dose response curve. [Supported by Boehringer Ingelheim.]

Peak flow criteria for hospital admission or discharge in acute
asthma

L DAVIES, I RYLAND, M ZAHIR, E KADZOMBE, MG PEARSON Aintree Chest Centre and the Accident and Emergency Department, Fazakerley Hospital, Liverpool The asthma guidelines recommend that patients with acute severe asthma with a peak flow $<50 \%$ of best or predicted value should be admitted. We studied retrospectively 100 consecutive 윽 patients (median age 33, range 5-68 years, 56\% female) presenting to casualty with acute asthma, recording basic patient data and $D$ hospital management. Sixty seven patients (group 1) were admitted, 을 31 (group 2) were discharged. Two were transferred to a paediatric hospital. Those admitted were older (median $36 v 23$ years), included more smokers $(57 \% v 29 \%)$, had more previous admissions $(56 \% v$ o $32 \%)$ and more on oral steroids $(36 \% v 10 \%)$ (all $\mathrm{p}<0.02)$. Use of inhaled bronchodilators $(82 \% v 80 \%)$ and inhaled steroids $(69 \% v<$ $61 \%$ ) was similar. All 18 patients with previous ITU admissions and all 16 with home nebulisers were admitted. Peak flows on arrival were recorded in 88 patients; nine of those without initial peak flows were subsequently admitted. Peak flows on arrival were lower $(\mathrm{p}<0.01)$ in those admitted (median $150 \mathrm{l} / \mathrm{min}$, range unrecordable-410) than in $\overrightarrow{\hat{\sigma}}$ those discharged (median $330 \mathrm{l} / \mathrm{min}$, range unrecordable-500) with a marked overlap between the groups (figure). More patients admitted

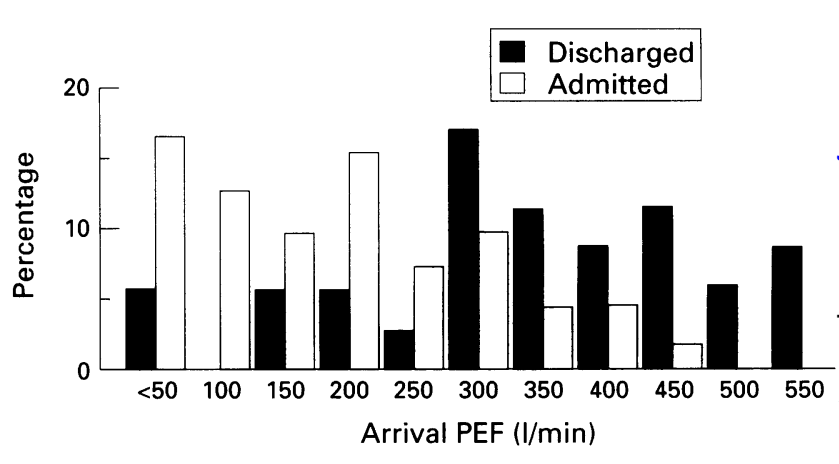

had a tachycardia $>110 / \mathrm{min}(33 \% v 17 \%)$ and were tachypnoeic (rate $>25)(55 \% v 41 \%)$. Of those sent home $5 / 31(16 \%)$ adults had a $\mathrm{PEF}<200 \mathrm{l} / \mathrm{min}$ on arrival. Four were female and two also had $\mathbb{D}$ tachycardia $>110 / \mathrm{min}$ and respiratory rate $>25$. Working to guidelines, $\stackrel{\infty}{+}$ these patients should have been admitted, which would result in $T$ $5 / 67(7 \%)$ more admissions. However none died or were readmitted $\overline{0}$ within two months. Whether the peak flow thresholds in the guidelines are adequate requires further evaluation.

\section{Follow up of asthma patients in hospital outpatient clinics}

I RYLAND, MG PEARSON on behalf of the NATIONAL ASTHMA TASK FORCE and 30 PARTICIPATING PHYSICIANS Aintree Chest Centre, Liverpool There are unsupported claims that hospital doctors follow up too many asthma patients in their clinics. The Asthma Task Force has surveyed 21 adult and nine paediatric hospitals collecting data on 894 adult and 401 paediatric patients. Median age of adults was 48 (13-93) years and of children $6(0.5-17)$ years. $59 \%$ of adults and $39 \%$ of children were female, and $65 \%$ adults and $62 \%$ children had 
had hospital admissions. Of the adults, $14 \%$ were on oral steroids, $24 \%$ on high dose inhaled ( \pm other therapy), $38 \%$ on low dose steroids ( \pm other therapy), and $24 \%$ on no anti-inflammatory treatment. For children $2 \%$ were on oral steroids, $20 \%$ on high dose inhaled steroids $25 \%$ low dose steroids, $10 \%$ cromoglycate, and $45 \%$ on no anti-inflammatory treatment. Main reasons given for requiring follow up of adults were asthma severity $431 / 740$ (58\%), concomitant disease $81 / 740(11 \%)$, and special patients (e.g. staff) $81 / 740(11 \%)$. For children these same reasons applied to $185 / 298(68 \%), 28 / 298(9 \%)$, and $19 / 298(6 \%)$ respectively. $112 / 740(15 \%)$ adults and $22 / 298(7 \%)$ children were discharged at that appointment with discharge being anticipated within six months for a further $17 \%$ and $16 \%$ respectively. Of $353 / 740(48 \%)$ of adults and $118 / 298(40 \%)$ of children in whom long term follow up was planned, these three reasons accounted for $90 \%$ of cases. $240 / 312(77 \%)$ adults and $83 / 131(63 \%)$ children being followed long term were on high dose steroids or more. When patients were asked who they would wish to be followed up by (if their asthma was well controlled), 338/708 (48\%) said GP alone, 290/708 (41\%) hospital and GP and only $80 / 708$ (11\%) hospital alone. This was independent of asthma severity as judged by treatment stage. Equivalent figures for paediatrics were $114 / 295$ (39\%), 125/295 (42\%) and $56 / 295(19 \%)$. Many patients would like to be followed up by GP alone. Hospital clinics seem to be discharging most mild asthma patients so that the majority of asthma patients being followed up long term do have severe disease. [Supported by a grant from the Department of Health.]

Increased nitric oxide in exhaled air of normal subjects with upper respiratory tract infection

SA KHARITONOV, DH YATES, PJ BARNES Department of Thoracic Medicine, National Heart and Lung Institute, London Nitric oxide (NO) is produced by a variety of cells within the lower respiratory tract, including inflammatory and epithelial cells, and is increased in inflammatory lung disorders. To determine whether exhaled NO is increased by upper respiratory tract infection (URTI) we measured exhaled NO in a total of 18 (11 men) normal subjects (mean age $33.7(1.05)$ years) during and after URTI, and compared values with age and sex-matched controls. All subjects with URTI had documented symptoms, such as generalised or frontal headache, myalgia, rhinorrhoea, sneezing, congestion/blocked nose, cough and chest pain accentuated by coughing ( 14 subjects) for at least two days prior to the investigation. Lung function parameters and exhaled nitric oxide (NO) were investigated in all subjects on two occasions 1-2 days after the beginning of URTI, and three weeks thereafter Exhaled NO was measured on a chemiluminescence analyser (Dasib Environmental Corporation, Glendale, California, USA). Subjects were asked to produce a slow vital capacity manoeuvre over 30-45 seconds into wide bore Teflon tubing. NO was sampled continuously The control group had been investigated earlier in the year and were used as a reference group regarding exhaled NO levels. Mean peak exhaled NO concentration was $87 \cdot 7(2 \cdot 67) \mathrm{ppb}$ in this group of 72 non-smoking controls (43 men; mean age $36 \cdot 1(2 \cdot 4)$ years). In subjects with URTI the peak was significantly higher $(314.6(57.03) \mathrm{ppb}$ $\mathrm{p}<0.001)$ during the acute phase of URTI, whereas peak NO values after recovery fell to $87.4(8.93) \mathrm{ppb}$, not significantly different from the normal controls. No change was observed in lung function. These data suggest that exhaled NO reflects both upper and lower respiratory tract inflammation and may have clinical utility in monitoring cytokine-mediated inflammation.

\section{Nitric oxide production by human bronchoalveolar lavage cells in culture}

PL LEUNG, K ANDERSON, C MCSHARRY, IB MCINNES, NC THOMSON, FY LIEW Department of Immunology and Respiratory Medicine, Westerm Infirmary, Glasgow There is increasing evidence from both animal and human studies suggesting that nitric oxide (NO) produced by inducible nitric oxide synthase (iNOS) may play an important role in the pathophysiology of certain lung diseases. We sought to establish whether cells obtained by lavage during diagnostic bronchoscopy can produce NO. Bronchoalveolar lavage (BAL) from 23 patients with mainly airways disease was included in this study. Cells from 19 patients (10 endobronchial cancer, three infection, six haemoptysis with negative bronchoscopy, age 29-84) were cultured in RPMI $5 \%$ FCS for 72-96 hours and NO production was measured by Greiss reaction. Expression of the iNOS gene was assessed by reverse transcription polymerase chain reaction (RT/PCR) using oligonucleotide primers corresponding to unique human iNOS sequence. In 15 of 19 samples there was no evidence of NO production after 72-96 hours in culture. The four samples (three bronchial carcinoma) which produced NO did so at low concentration (range $7-13 \mathrm{nmol} / 10^{6} \mathrm{cells} / \mathrm{ml}$ ) and was not increased even after stimulation with LPS and SEB; this NO production was inhibited in the presence of $5 \mathrm{mM}$ L-NMMA. iNOS mRNA was detected in four of 10 patients and in one patient NO was detected but PCR was negative. The majority of BAL samples did not produce NO either spontaneously or after stimulation. The few positive were mainly from subjects with malignant airways disease.

\section{L-Arginine increases exhaled nitric oxide in normal subjects}

SA KHARITONOV, G LUBEC, B LUBEC, M HJELM, PJ BARNES Department of Thoracic Medicine, National Heart and Lung Institute, London; University Hospital, Vienna, Austria; Hospital for Sick Children, London We have investigated the effect of orally administered L-arginine $(0 \cdot 05,0 \cdot 1$, $0.2 \mathrm{~g} / \mathrm{kg}$ ) compared with matched placebo on the concentration of nitric oxide (NO) in the exhaled air in 23 normal individuals. Administration of the dose of L-arginine (or placebo) was designated time 0 and exhaled NO was measured prior to medication, then every 30 minutes up to five hours after medication. A blood sample was taken for measurement of nitrate and arginine prior to dosing then two hours after dosing. Heart rate, supine blood pressure, and FEV were measured hourly throughout the study. The doses of L-arginine $0.1 \mathrm{~g} / \mathrm{kg}(\mathrm{n}=7)$ and $0.2 \mathrm{~g} / \mathrm{kg}(\mathrm{n}=4)$, but not $0.05 \mathrm{~g} / \mathrm{kg}(\mathrm{n}=4)$, induced a significant increase in exhaled NO two hours after administration (baseline $64(7 \cdot 26)$ ppb; two hours $97(4 \cdot 88) \mathrm{ppb}, 52(6 \cdot 59) \mathrm{ppb}$, and $156(19 \cdot 3) \mathrm{ppb}$, respectively) which appeared to be dose-related. This was paralleled by a dose-related increase in plasma nitrate and arginine concentrations two hours after administration. There was no effect of placebo (five subjects) on the levels of exhaled NO over the same time period, nor on plasma nitrate or arginine concentrations. The increase in exhaled NO was maximal at two hours and then declined by 3-4 hours to baseline levels. Calculation of the area under the curve by planimetry demonstrated a dose-related increase $(r=0.93$, $\mathrm{p}<0.001)$ in exhaled NO $(10 \cdot 2(6.6)$ arbitrary units at $0.05 \mathrm{~g} / \mathrm{kg}, 41.1$ $(8 \cdot 8)$ units at $0 \cdot 1 \mathrm{~g} / \mathrm{kg}$, and $114.5(24 \cdot 0)$ units at $0.2 \mathrm{~g} / \mathrm{kg})$. There was no significant effect on heart rate, blood pressure, or $F E V_{1}$ over the period of measurement. The results suggest that an increase in the amount of substrate for NO synthase can increase the formation of endogenous NO.

\section{Downregulation of the constitutive nitric oxide synthase (NOS)} gene expression in rats treated with lipopolysaccharide in vivo

SF LIU, IM ADCOCK, PJ BARNES, TW EVANS Unit of Intensive Care, Department of Thoracic Medicine, National Heart and Lung Institute, London Bacterial endotoxin has been reported to be both stimulatory and inhibitory on nitric oxide release. One explanation for this discrepancy is that endotoxin may affect the constitutive and inducible NOS (iNOS) gene differently. We have reported that lipopolysaccharide (LPS) treatment of rat in vivo causes a widespread tissue expression of the iNOS gene. We now study the effects of LPS and dexamethasone (Dexa) treatment in vivo on the endothelial type (eNOS) and brain type (bNOS) NOS mRNA expression. The glyceraldehyde phosphate dehydrogenase (GAPDH) gene was also studied in parallel as an internal standard. Wistar rats were treated with either saline ( $1 \mathrm{ml} / \mathrm{kg}$ ip, control group) or LPS $(15 \mathrm{mg} / \mathrm{kg}$ ip, LPS group) for four hours. For LPS + Dexa group, Dexa $(3 \mathrm{mg} / \mathrm{kg}$ ip) was injected 40 minutes prior to the administration of LPS. Lung, heart, aorta and brain were dissected from these animals. Total and mRNA were isolated using commercial kit. Northern blot analysis was performed according to standard methods. The eNOS and bNOS mRNA level were quantified using densitometry and expressed as eNOS/GAPDH or bNOS/GAPDH ratio. Neither LPS nor Dexa had any effect on the GAPDH mRNA levels. The eNOS/GAPDH ratios for control, LPS, and LPS + Dexa groups, respectively, were 0.31 $(0.07), 0 \cdot 11(0.04)$, and $0.08(0.02)(\mathrm{p}<0.03$ compared with control, $\mathrm{n}=6)$ in the lung, and $0.16(0.03), 0.04(0.02)$, and $0.06(0.02)$ $(\mathrm{p}<0.03$ compared with control, $n=6$ ) in heart. Similar results were obtained from aorta. The bNOS/GAPDH ratio was $0.42(0 \cdot 15), 0 \cdot 10$ $(0.02)$, and $0.06(0.01)(p<0.03$ compared with control) for control, Endo, and Endo + Dexa group respectively. These results demonstrate that LPS treatment in vivo downregulates the two isoforms of con- 
stitutive NOS mRNA expression, which may also contribute to the multi-organ dysfunction seen as septic shock. [Supported by British Heart Foundation.]

Decreased endogenous nitric oxide in the exhaled air of systemic sclerosis patients with pulmonary hypertension

JB CAILES, S KHARITONOV, D YATES, P BARNES, RM DUBOIS Royal Brompton Hospital, London Systemic sclerosis (SSc) may be complicated by pulmonary hypertension (PHT) which can occur both in the setting of fibrosing alveolitis (FASSc) or as lone pulmonary vascular disease. PHT is now recognised as the most common cause of death in SSc. Nitric oxide (NO) is produced by a variety of cells in the lower respiratory tract including pulmonary vascular endothelial cells and can be measured in expired air. NO is both an inhibitor of platelet aggregation and a powerful vasodilator which has been used therapeutically to treat PHT. We aimed to test the hypothesis that exhaled NO levels would be decreased in SSc patients with PHT. We measured exhaled breath NO levels by a chemiluminescence analyser in 24 patients with SSc, (mean (SD) age 52 (11) years, 17 women). Presence or absence of PHT was determined by Doppler echocardiography performed median (interquartile range) $2 \cdot 6(0-10 \cdot 5)$ months prior to the estimation of NO. Five patients had PHT, four of whom $(80 \%)$ had previously had this confirmed invasively by right heart catheterisation. Full lung function was performed at the same visit as measurement of NO, and thin section CT scans were analysed for the presence of abnormality consistent with fibrosing alveolitis. Exhaled NO (ppb) was significantly decreased in SSc complicated by PHT from a median (IQ range) of 150 (72-211) in patients without PHT to $19.5(13-32)(\mathrm{p}=0.001)$. SSc patients with PHT were also significantly older $(p=0.03)$ and had decreased alveolar to arterial gradients for oxygen (Aa) and gas transfer (TLCO) $(p=0.03$ and 0.05 , respectively). When controlling for the effect of age, Aa, and TLco by multiple linear regression analysis PHT was the only factor which was independently associated with NO levels $(p=0.03)$. No association was found between the presence of a CT scan with abnormality consistent with fibrosing alveolitis and NO levels $(p=1 \cdot 0)$. We conclude that exhaled breath $\mathrm{NO}$ is decreased in SSc patients with PHT. Decreased NO levels may reflect endothelial dysfunction and be involved in the pathogenesis of the obliterative pulmonary vascular disease seen in this condition. [Supported by a grant from the Raynauds and Scleroderma Association UK.]

\section{Hypoxic constrictor response in intrapulmonary artery rings from chronic hypoxic rats}

VSNMR KARAMSETTY, RM WADSWORTH, KA KANE, MR MACLEAN, KM MCCULLOCH Department of Physiology and Pharmacology, University of Strathclyde, Glasgow; Institute of Physiology, Glasgow University, Glasgow Hypoxic constrictor response (HCR) has been reported to be either blunted or augmented in isolated lungs from chronic hypoxic (CH) animals. However, there are no reports of HCR in isolated pulmonary arteries from $\mathrm{CH}$ animals. The aim of this study was to investigate the effects of acute hypoxia in isolated intrapulmonary arteries (IPA) from CH Wistar rats (hypobaric hypoxia, two weeks). Isometric tension was recorded from IPA rings from the control (C) and $\mathrm{CH}$ rats (internal diameter $2.14(0.07)$ v $2.43(0.08) \mathrm{mm}$ respectively, $\mathrm{p}<0.05)$. Once the active tone induced by phenylephrine (PE) became stable, hypoxia $(\mathrm{H})$ was introduced by bubbling organ chambers with $95 \% \mathrm{~N}_{2}+5 \% \mathrm{CO}_{2}$ for 30 minutes. Hypoxia produced an initial rapid contraction which was reduced in IPA rings from $\mathrm{CH}$ compared with C rats $\left(50.0(4.8) v 30.5(6.5) \mathrm{g} / \mathrm{cm}^{2}, \mathrm{p}<0.05\right)$. This was followed by a partial relaxation $\left(11.4(4 \cdot 1) v 9 \cdot 0(7 \cdot 8) \mathrm{g} / \mathrm{cm}^{2}\right.$, $\mathrm{p}>0.05)$ and a slowly developing sustained contraction $(39.2(6.4) v$ $\left.28.3(10) \mathrm{g} / \mathrm{cm}^{2}, \mathrm{p}>0.05\right)$ in IPA rings from $\mathrm{C}$ and $\mathrm{CH}$ rats respectively. Carbachol induced relaxation was impaired in IPA rings from $\mathrm{CH}$ $(56(4.4) \%)$ compared with $\mathrm{C}$ rats $(88(2 \cdot 5) \%, \mathrm{p}<0.05)$. Removal of endothelium resulted in a loss of the first $\left(2.59(1 \cdot 11) \mathrm{g} / \mathrm{cm}^{2}\right)$ and third $\left(-5.07(1.76) \mathrm{g} / \mathrm{cm}^{2}\right)$ phases of hypoxic contraction in IPA rings from both $\mathrm{C}$ and $\mathrm{CH}$ rats. Similarly, incubating with $\mathrm{N} \omega \mathrm{Nitro}-\mathrm{L}-$ Arginine (LNOARG, $3 \times 10^{-4} \mathrm{M}$ ) resulted in a complete loss of the first and third phases of hypoxic contraction and hypoxia resulted in only a relaxation. Removal of the endothelium or treatment with LNOARG had no effect on the hypoxic phase of relaxation in IPA rings from $\mathrm{C}$ and $\mathrm{CH}$ rats. These results show that the first phase only of HCR was slightly blunted in $\mathrm{CH}$ rat IPA rings and that the contractile phases, but not the relaxation, were endothelium/NO dependent.

\section{Hypoxic pulmonary vasoconstriction/dilatation is modified by} different preconditions

GQ TENG, GR BARER Department of Medicine, Royal Hallamshire Hospital, Sheffield In vitro, hypoxia causes both constriction and di- $\overrightarrow{\overline{\vec{B}}}$ latation. Small vessels were mounted in a Cambustion myograph in physiological saline in the isometric mode, gassed with $\mathrm{O}_{2}$ or $\mathrm{N}_{2}+5 \% \mathrm{CO}_{2}$. After preconstriction with $4 \mu \mathrm{g} \mathrm{PGF}_{2 \alpha}$ hypoxia caused a four-phase response: (1) dilatation; (2) constriction; (3) dilatation; $\frac{\bar{S}}{\supset}$ and (4) a slow small constriction. This response was repeatable. The $\mathbb{\unrhd}$ response varied with the level of preconstriction and with different agonists. In 20 vessels (five rats), at lower levels of preconstriction with $\mathrm{PGF}_{2 x}$, hypoxia caused small prolonged constrictions only; both $\vec{\circ}$ dilator and constrictor responses increased with the level of pre- $\overrightarrow{\vec{H}}$ constriction. During $\mathrm{KCl}$ preconstriction, dilatation and constriction $\vec{\omega}$ were less than after $\mathrm{PGF}_{2 \alpha}$ (phase $1,0.017(0.01) v 0.21(0.02) \mathrm{mN} /$ $\mathrm{mm}$; phase 2, $0.33(0.097)$ v $2.19(0.21) \mathrm{mN} / \mathrm{mm}$, both $\mathrm{p}<0.001), \vec{x}$ even though the preconstriction level was similar $\left(\mathrm{PGF}_{2 \alpha} 0.99 \mathrm{mN} /\right.$ of $\mathrm{mm} ; \mathrm{KCl} 0.91 \mathrm{mN} / \mathrm{mm})$. At $25^{\circ} \mathrm{C}$ the maximal constriction of eight pulmonary vessels, (four rats) to $\mathrm{KCl}$ was unchanged $(3.35(0.91) \mathrm{mN} /$ i $\mathrm{mm} v 3.71(0.99) \mathrm{mN} / \mathrm{mm}, \mathrm{p}=0.2661)$ compared with $38^{\circ} \mathrm{C}$, but $\mathrm{PGF}_{2 x}$ maximal contraction was reduced $(1.48(0.32) \mathrm{mN} / \mathrm{mm} v 3.08$ 七 $(0.61) \mathrm{mN} / \mathrm{mm}, \mathrm{p}<0.001)$. Acetylcholine $(\mathrm{ACH})$ relaxation was higher $\frac{\mathrm{O}}{3}$ at $25^{\circ} \mathrm{C} \quad(66.57 \% \quad(12 \cdot 16) \%$ v $38.93 \quad(7.62) \%$ precontraction, $\mathrm{p}<0.001)$. The hypoxic response was tested at $25^{\circ} \mathrm{C}$ in eight vessels (four rats) precontracted with $\mathrm{PGF}_{2 \alpha}$. The magnitude of contraction by hypoxia compared with $38^{\circ} \mathrm{C}$ was unchanged. However, at $25^{\circ} \mathrm{C}$ 工 the onset of hypoxic constriction was delayed $(2 \cdot 83(0.6) \mathrm{min}$ at $38^{\circ} \mathrm{C}$ and $6.87(1.24) \mathrm{min}$ at $\left.25^{\circ} \mathrm{C}, \mathrm{p}<0.005\right)$. The contraction was of maintained for a longer time $\left(7.83(1.64) \mathrm{min}\right.$ at $38^{\circ} \mathrm{C} v 24.62$ $(4 \cdot 34) \mathrm{min}$ at $\left.25^{\circ} \mathrm{C}, \mathrm{p}<0.001\right)$, and dilatation was absent.

Energy state, intracellular pH and tension responses in pulmonary artery rings during hypoxia and absence of substrate

RM LEACH, DW SHEEHAN, VP CHACKo, JT SYLVESTER Department of Intensive Care, St Thomas' Hospital, London Reduction in energy state may trigger hypoxic pulmonary vasoconstriction and is associated with hypoxic systemic vasorelaxation. ${ }^{31} \mathrm{P}$ nuclear magnetic resonance spectroscopy (NMR) was used to measure phosphocreatine (Pcr), ATP and intracellular $\mathrm{pH}(\mathrm{pHi})$ in porcine intrapulmonary artery rings. Paired rings were stretched to $4 \mathrm{~g}$ tension in standard organ 3 baths. One ring from each pair was transferred to a plastic frame at its stretched length, placed in a sample tube within the bore of an 11.8 Tesla MSL 500 NMR spectrometer, and superfused with phosphate-free Krebs-bicarbonate solution $\left(37^{\circ} \mathrm{C}, \mathrm{pH}=7 \cdot 4\right)$ con- 윽 taining phenylephrine $\left(10^{-7} \mathrm{M}\right)$. NMR spectra were obtained from $D$ free induction decays collected over 15 minutes. The other ring remained in the organ bath to allow measurements of tension and was subjected to identical conditions. Experiments consisted of control, of experimental and recovery periods each two hours in length. In the $N$ control and recovery periods the perfusate contained glucose $(10 \mathrm{mM}) \mathrm{N}$ and was gassed with $93 \% \mathrm{O}_{2}$. In the experimental period the perfusate $\omega$ was either gassed with $0 \% \mathrm{O}_{2}\left(\mathrm{Po}_{2}<20\right.$ torr $)$ or glucose was removed (sucrose substitution) or both. During hypoxia Pcr transiently fell to $37(14 \%)$ of baseline returning to $90(10) \%$ at two hours. Initially $\mathbb{D}$ pHi also fell from the control value of $7.24(0.02)$ to $7.08(0.03)$ recovering to $7 \cdot 18(0.02)$ after two hours hypoxia. ATP was unaffected. These changes correlated with initial vasorelaxation followed by vaso-

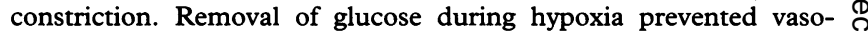
constriction, inhibited the recovery of $\mathrm{Pcr}$ and $\mathrm{pHi}$, and resulted in a fall in ATP. Absence of glucose without hypoxia had no effect on tension, energy state, or pHi. The results suggest a link between energy state, pHi and pulmonary vasomotor tone. [RML supported by MRC.]

Endothelin receptors in rat pulmonary arteries and arterioles: effect of pulmonary hypertension

KM MCCULLOCH, M BAIRD, MR MACLEAN Division of Neuroscience and Biomedical Systems, IBLS, West Medical Building, Glasgow University Glasgow In adult rats vasoconstrictor responses to endothelin-1 (ET- 
1) are mediated via the $\mathrm{ET}_{\mathrm{A}}$ receptor in pulmonary arteries (PAs) but through both $\mathrm{ET}_{\mathrm{A}}$ and $\mathrm{ET}_{\mathrm{B}}$ receptors in pulmonary resistance arteries (PRAs) (MacLean et al. $\mathcal{F}$ Cardiovasc Pharmacol 1994;23:838-45). Here we investigated the effect of pulmonary hypertension $(\mathrm{PH})$ induced by exposure to hypoxia on PA and PRA responses to ET-1 in young SPF rats. Chronic hypoxic $(\mathrm{CH})$ rats (28-30 days old) exposed to $10 \% \mathrm{O}_{2}$ for two weeks and age matched controls were studied. The first branch pulmonary arteries (PAs) were set up under $1.5 \mathrm{~g}$ tension in $5 \mathrm{ml}$ organ baths. The PRAs $(150 \mu \mathrm{m}$ id) were set up on a wire myograph under $130-40 \mathrm{mg}$ tension. All vessels were bubbled with $16 \% \mathrm{O}_{2} / 6 \% \mathrm{CO}_{2}$. Response curves were obtained to ET-1 ( \pm the $\mathrm{ET}_{\mathrm{A}}$ antagonist FR $139317[\mathrm{FR}, 1$ and $\left.10 \mu \mathrm{M}]\right)$ and the $\mathrm{ET}_{\mathrm{B}}$ agonist sarafotoxin S6c (SXS6c) ( $n=5-10$ rings in 6-10 rats). Control rat PAs contracted only to ET-1 $\left(\log \mathrm{M} \mathrm{EC}_{50}\right.$ : -8.4 $(0 \cdot 2))$ and these responses were inhibited by FR139319, indicating contraction via the $\mathrm{ET}_{\mathrm{A}}$ receptor only. $\mathrm{CH}$ rats contracted to both ET-1 (which was more potent than in controls, $p<0.05$, Student's unpaired $t$ test, $\log \mathrm{M} \mathrm{EC}_{50}$ : $\left.-8.9(0 \cdot 1)\right)$ and SXS6c $\left(\log \mathrm{M} \mathrm{EC}_{50}\right.$ : $-7 \cdot 7(0 \cdot 2)$, maximum response: $30 \%$ maximum to ET-1) indicating contraction through the $\mathrm{ET}_{\mathrm{A}}$ receptor and an $\mathrm{ET}_{\mathrm{B}}$-like receptor. $\mathrm{FR}$ sensitive responses to ET-1 were seen in both control and CH PRAs ( $\log \mathrm{M} \mathrm{EC}_{50}$ controls: $-8 \cdot 1(0 \cdot 1), \mathrm{CH}$ rats: $\left.-8 \cdot 1(0 \cdot 1)\right)$. SXS6c also induced potent contractions in the PRAs $\left(\log \mathrm{M} \mathrm{EC}_{50}\right.$ controls: $-9 \cdot 2$ $(0 \cdot 1), \mathrm{CH}$ rats: $-8.9(0 \cdot 1))$. The maximum response to ET-1 was increased in the $\mathrm{CH}$ rats by $50 \%$ whilst the maximum response to SXS6c was unchanged. This study shows that ET-1 contraction is mediated only by $\mathrm{ET}_{\mathrm{A}}$ receptors in these rat PAs but that $\mathrm{ET}_{\mathrm{B}}$ receptors play a major role in PRAs. In PAs, PH induced an increase in the $\mathrm{ET}_{\mathrm{A}}$-mediated response to $\mathrm{ET}-1$ and uncovered an $\mathrm{ET}_{\mathrm{B}}$-mediated component. In PRAs, $\mathrm{PH}$ induced an increase in the $\mathrm{ET}_{\mathrm{A}}$-mediated response. Hence, ET receptor expression is modified by $\mathrm{PH}$ in rats.

\section{Cyclic GMP levels are raised in endothelium-denuded rat} pulmonary artery after LPS treatment

MJD GRIFFITHS, M MESSENT, NP CURZEN, TW EVANS National Heart and Lung Institute, London Nitric oxide (NO) acts on vascular smooth muscle through activation of soluble guanylyl cyclase and elevation of guanosine 3',5'-cyclic monophosphate (cGMP) (Circ Res 1983;52: 352-7). We have demonstrated pharmacologically that endotoxin (LPS) injection induces NO synthase (NOS) in rat pulmonary vascular smooth muscle (PVSM) (Thorax 1993;48:467). Biochemical confirmation of NOS induction in PVSM requires the demonstration of raised levels of cGMP in vessels from LPS-treated rats that could be decreased by an NOS inhibitor, eg. $\mathrm{N}^{\mathrm{G}}$-monomethyl N-arginine (LN). Male Wistar rats were treated with LPS $(20 \mathrm{mg} / \mathrm{kg}$ ip) or vehicle $(1 \mathrm{ml} \mathrm{NaCl}$, ip) four hours before sacrifice. Main pulmonary artery rings were suspended in organ baths containing oxygenated Krebs solution. Their endothelium was removed by abrasion, confirmed by failure to relax to acetylcholine after construction of a phenylephrine (PE) dose-response curve. After equilibration, vessels were contracted by their $\mathrm{EC}_{50} \mathrm{PE}$ and then by $\mathrm{LN}(50 \mu \mathrm{M}$ or $1 \mathrm{mM})$ or vehicle until a plateau tension was reached when the rings were snap frozen and stored at $-80^{\circ} \mathrm{C}$ prior to radioimmunoassay for cGMP (Adv Cyclic Nucl Res 1979;10:1-33). Results shown in the table are the mean (SE)

\begin{tabular}{|c|c|c|c|c|c|}
\hline \multirow[b]{2}{*}[LN]{} & \multicolumn{2}{|l|}{ Sham } & \multicolumn{3}{|l|}{ LPS } \\
\hline & 0 & $1 \mathrm{mM}$ & 0 & $50 \mu M$ & $1 m M$ \\
\hline $\begin{array}{l}\mathrm{T}_{\mathrm{LN}}(\%) \\
\mathrm{cGMP} \\
(\mathrm{fmol} / \mathrm{mg})\end{array}$ & $\begin{array}{l}90(4) \\
20(3)\end{array}$ & $\begin{array}{l}93(5) \\
16(3)\end{array}$ & $\begin{array}{l}94(2) \\
52(5)\end{array}$ & $\begin{array}{r}170(18) \\
33(5)^{*}\end{array}$ & $\begin{array}{r}226(20)^{* * *} \\
20(3)^{* *}\end{array}$ \\
\hline
\end{tabular}

${ }^{*} \mathrm{p}<0.05,{ }^{* *} \mathrm{p}<0 \cdot 01 \mathrm{LN}[0] v \mathrm{LN}[50 \mu \mathrm{M}$ or $1 \mathrm{mM}]$. $\mathrm{LN}$-induced tension $\left(\mathrm{T}_{\mathrm{LN}}\right)$ expressed as \% contraction caused by the
$\mathrm{PE} \mathrm{EC} \mathrm{EC}_{50}$, and the cGMP level ( $\left.\mathrm{fmol} / \mathrm{mg}, \mathrm{n}=8\right)$. cGMP levels were increased following LPS treatment and diminished in a dose-dependent manner by NOS inhibition, suggesting that NO activity was increased following NOS induction in PVSM by LPS. [MG is a Wellcome Clinical Research Fellow.]
Endothelin-1 induced contraction in human pulmonary arteries: mediated by $\mathbf{E T}_{\mathrm{A}}$ receptors in large arteries but non$\mathrm{ET}_{\mathrm{A}} /$ non-ET $\mathrm{T}_{B}$ receptors in resistance arteries

KM MCCULLOCH, M BAIRD, MR MACLEAN Division of Neuroscience and Biomedical Systems, IBLS, West Medical Building, Glasgow University, Glasgow In rats vasoconstrictor responses to endothelin-1 (ET-1) are mediated via the $\mathrm{ET}_{\mathrm{A}}$ receptor in pulmonary arteries (PAs) but through both $\mathrm{ET}_{\mathrm{A}}$ and $\mathrm{ET}_{\mathrm{B}}$ receptors in pulmonary resistance arteries (PRAs) (MacLean et al. f Cardiovasc Pharmacol 1994;23:838-45). Here we have used ET-1 (ET ${ }_{A}$ and $\mathrm{ET}_{\mathrm{B}}$ receptor agonist), sarafotoxin S6c (SXS6c, selective ET ${ }_{B}$ agonist), FR 139317 (FR, selective ET $_{A}$ antagonist) and bosentan (non-selective $\mathrm{ET}_{\mathrm{A}}$ and $\mathrm{ET}_{\mathrm{B}}$ antagonist) to determine the ET receptors involved in ET-1 induced vasoconstriction in human PAs and PRAs. Ring preparations of intrapulmonary arteries, 2-3 mm id (PAs) and 200-300 $\mu \mathrm{m}$ id (PRAs), were dissected from healthy sections of human postoperative bronchial carcinoma tissue. The PAs were mounted in $5 \mathrm{ml}$ organ baths in Krebs under $2 \mathrm{~g}$ tension whilst the PRAs were mounted on a wire myograph under $125 \mathrm{mg}$ tension. Vessels were bubbled with $16 \% \mathrm{O}_{2} / 6 \% \mathrm{CO}_{2}$ balance $\mathrm{N}_{2}$. Response curves were constructed to ET-1 and SXS6c ( $3 \mathrm{pM}-1 \mu \mathrm{M})$. A maximal dose of ET-1 was administered after the last concentration of SXS6c. Responses to ET-1 and SXS6c were constructed in the absence or presence of FR 139317 or bosentan $(1 \mu \mathrm{M}$ and $10 \mu \mathrm{M})$. The PAs did not contract to SXS6c but did contract to ET-1 (logM $\mathrm{EC}_{50}$ : $-8 \cdot 3(0 \cdot 2),[\mathrm{n}=9$ rings/9 lungs] $)$ and these contractions were inhibited by both FR and bosentan indicating the responses are mediated via the $\mathrm{ET}_{\mathrm{A}}$ receptor subtype. The PRAs, however, contracted to both ET-1 $\left(\log \mathrm{EC}_{50}:-8 \cdot 7(0 \cdot 3)\right.$ [ $\mathrm{n}=9$ rings/8 lungs] $)$ and SXS6c (max response: $30 \%$ that to ET-1; $\log \mathrm{EC}_{50}$ : $-10 \cdot 8$ $(0 \cdot 3),[n=7$ rings/ 3 lungs] $)$. The responses to ET- 1 were not, however, inhibited by FR indicating contraction mediated by a non-ET $\mathrm{A}_{\mathrm{A}} /$ non$\mathrm{ET}_{\mathrm{B}}$ receptor similar to those described in human coronary arteries (Godfraind BrF Pharmacol 1993;110:1201-5). [This work was funded by Tenovus and the MRC.]

Responses to 5HT and sumatriptan in control and pulmonary hypertensive rats

MR MCLEAN, M BAIRD, KM MCCULLOCH CRI Laboratories, Division of Neuroscience and Biomedical Systems, IBLS, West Medical Building, Glasgow University, Glasgow We have previously shown that contractile $5 \mathrm{HT}_{1 \mathrm{D}}$ receptor-mediated responses to sumatriptan exist in bovine and human pulmonary arteries and that in these vessels, unlike in the systemic circulation, a substantial component of the contractile response to $5 \mathrm{HT}$ is mediated via the $5 \mathrm{HT}_{1 \mathrm{D}}$ receptor (MacLean et al. Pulmon Pharmacol 1994;7:65-72; Templeton et al. Thorax 1994; 49:425P). Here we investigated the effect of pulmonary hypertension induced by chronic hypoxia on pulmonary arterial responses to 5HT and sumatriptan in rats. Chronic hypoxic $(\mathrm{CH})$ rats exposed to $10 \%$ $\mathrm{O}_{2}$ for two weeks and their age matched controls were studied. Pulmonary hypertension was confirmed by measuring right ventricular/total ventricular weight ratio which was $60 \%$ greater in the $\mathrm{CH}$ rats. The right branch pulmonary arteries (PAs) were set up as ring preparations under $1.5 \mathrm{~g}$ tension in organ baths, in Krebs solution bubbled with $16 \% \mathrm{O}_{2} / 6 \% \mathrm{O}_{2}$. Cumulative concentration response curves (CCRCs) were constructed to both 5HT and sumatriptan. In the latter experiments, a maximum concentration of $5 \mathrm{HT}$ was administered at the end of the CCRC to sumatriptan. Responses to $5 \mathrm{HT}$ were greater in the PAs from the $\mathrm{CH}$ rats, the $\log \mathrm{MEC}_{50}$ values were $-6 \cdot 1(0 \cdot 2)(n=6)$ in control PAs and $-7 \cdot 6(0 \cdot 1)(n=6)$ in $\mathrm{CH}$ rat PAs ( $<<0.001$ compared with controls; Student's paired $t$ test). In the control PAs there was no response to sumatriptan whereas the $\mathrm{CH}$ rat PAs contracted to sumatriptan which gave a maximum response $40-50 \%$ of that to $5 \mathrm{HT}$. The $\log \mathrm{M} \mathrm{EC}_{50}$ value for sumatriptan was $-6.0(0.5)(n=6)$ and therefore equipotent with $5 \mathrm{HT}$ in the control PAs. In conclusion, not only are $5 \mathrm{HT}_{1 \mathrm{D}}$-induced contractile responses important in PAs, but this study suggests that they are potentiated in pulmonary hypertension and may account for the increased response to $5 \mathrm{HT}$ observed in $\mathrm{CH}$ rats. [This work was funded by the MRC.]

Vasodilator efficacy of anitrosothiol SNAP and SNP in normal and chronic hypoxic rat lung

CELIA EMERY Department of Medicine, University of Sheffield, Sheffield s-Nitrosothiols, which liberate nitric oxide, may mediate 
endothelial-dependent vasodilatation. The effect of s-nitroso acetyl penicillamine (SNAP) on pulmonary vascular tone (low during normoxia or raised by either chronic hypoxia $\left(10 \% \mathrm{O}_{2}\right.$ three weeks) or during acute hypoxic vasoconstriction (HPV)) was tested in isolated rat lungs (IPL) and compared with the effect of SNP, sodium nitroprusside, a non-endothelial dependent dilator. Lungs were perfused in situ with homologous blood at a constant flow of $20 \mathrm{ml} / \mathrm{min}$; and ventilated with either air (normoxia) or $2 \% \mathrm{O}_{2}$ (hypoxia), both $+5 \% \mathrm{CO}_{2}$. Effect of $0 \cdot 1 \mathrm{ml}$ bolus doses of SNAP or SNP $(0 \cdot 01,0 \cdot 1$, $1,10,100 \mu \mathrm{g})$ on pulmonary artery pressure (Ppa) was studied in both normal (C) and chronically hypoxic (CH) IPLs. During normoxia both SNAP and SNP caused small falls in Ppa, being more obvious in $\mathrm{CH}$ (range $0.5-3.5 \mathrm{~mm} \mathrm{Hg}$ ). During hypoxia SNAP and SNP caused dose-dependent falls in Ppa, expressed as \% fall in HPV, similar in $\mathrm{C}$ and $\mathrm{CH}$ rats. However, SNP was 10 times more potent than SNAP ( $100 \%$ fall in HPV with $100 \mu \mathrm{g}$ SNAP $v 10 \mu \mathrm{g}$ SNP). The nitric oxide synthase inhibitor, L-NAME, had no consistent effect

Comparison of \% fall in HPV with SNAP and SNP in $C$ and $C H$ rat IPLs (mean (SD))

\begin{tabular}{lllllll}
\hline & $0 \cdot 1 \mu g$ & $0 \cdot 1 \mu g$ & $1 \mu g$ & $1 \mu g$ & $10 \mu g$ & $10 \mu g$ \\
& $S N A P$ & $S N P$ & $S N A P$ & $S N P$ & $S N A P$ & $S N P$ \\
\hline $\mathrm{C}$ & $18(12)$ & $23(16)$ & $28(12) \dagger$ & $64(24)^{*} \dagger$ & $57(21)^{*}$ & $102(12)^{*} \dagger$ \\
$\mathrm{CH}$ & $9(5)$ & $18(25)$ & $28(14)^{*}$ & $53(9)^{*} \dagger$ & $57(16)^{*}$ & $97(21)^{*} \dagger$ \\
\hline
\end{tabular}

*Sig. diff from previous dose.

† Sig. diff SNAP $v$ SNP same dose.

on the vasodilatation. Thus, the nitrosothiol, SNAP, causes profound vasodilatation in the rat lung, equipotent in $\mathrm{C}$ and $\mathrm{CH}$, although potency was less than SNP. Neither were consistently affected by blockade of endogenous NO release. Inhaled nitrosothiols may provide an alternative treatment to nitric oxide in pulmonary hypertension.

\section{Nebulised prostacyclin used as a selective pulmonary vaso-} dilator in acute lung injury

JN SHEPHARD, SJ BRETT, B KEOGH, TW EVANS Unit of Critical Care, Royal Brompton Hospital, London Prostacyclin $\left(\mathrm{PGI}_{2}\right)$ has been widely used intravenously as a vasodilator in a variety of pathological circumstances, including sepsis and pulmonary hypertension. There are theoretical and practical advantages to using a selective and locally administered vasodilator in the management of pulmonary hypertension and hypoxia in patients with ARDS. We describe the response to nebulised $\mathrm{PGI}_{2}$ of four patients with ARDS secondary to a variety of pathologies. Prior to $\mathrm{PGI}_{2}$ administration they were managed on pressure controlled, inverse ratio ventilation, which was continued during therapy. Prostacyclin was administered via a gasdriven nebuliser attached to the distal portion of the inspiratory limb of the ventilator circuit. Baseline measurements of pulmonary (PVR), systemic (SVR), vascular resistance and $\mathrm{PaO}_{2} / \mathrm{FIO}_{2}$ ratio (P/F) were made. The response to increasing doses of $\mathrm{PGI}_{2}$ were then assessed. The results are shown in the table as \% changes from baseline. The

\begin{tabular}{llll}
\hline & Peak & Mean $(n=4)$ & Range \\
\hline PVR & $-24 \%$ & $-15 \cdot 5 \%$ & $8-24 \%$ \\
SVR & $-14 \%$ & $-8 \cdot 2 \%$ & $2-14 \%$ \\
P/F ratio & $+67 \%$ & $+48 \cdot 5 \%$ & $+14-67 \%$ \\
\hline
\end{tabular}

minimum effective dose ranged from 4 to $32 \mathrm{ng} / \mathrm{kg} / \mathrm{min}$. These data show that $\mathrm{PGI}_{2}$ may be successfully administered via nebuliser to patients with ARDS. Its effects were to reduce intrapulmonary shunt fraction and PVR without significantly changing SVR. The wide variation in dose response between patients shows the value of performing a dose response for each patient.

\section{Prediction of pulmonary hypertension in patients with systemic} sclerosis and fibrosing alveolitis

JB CAILES, CM BLACK, RM DUBOIS Royal Brompton Hospital, London Systemic sclerosis (SSc) is often associated with fibrosing alveolitis (FASSc). Pulmonary hypertension (PHT) is the major cause of mortality in this condition. Precise estimation of pulmonary artery pressure requires the invasive procedure of right heart catheterisation. Doppler echocardiography (DE), however, is both sensitive and specific for the diagnosis of PHT in SSc but this test is not routinely available in all hospitals. We aimed to identify the demographic, disease and lung function factors which would most reliably predict risk of PHT in patients with FASSc. One hundred and thirty patients with FASSc prospectively underwent Doppler echocardiography. PHT was diagnosed if Doppler estimated pulmonary artery systolic ఖ্ pressure was greater than $30 \mathrm{~mm} \mathrm{Hg}$ (29 patients). Exclusion of PHT ? required demonstration of a structurally normal right ventricle and a $\overrightarrow{\overline{\vec{A}}}$ normal time interval between closure of the pulmonary valve and start of tricuspid flow on Doppler. Patients with evidence of pericardial disease or reduced left ventricular function were excluded leaving 113 patients for analysis. Demographic data (age, sex, and smoking $\frac{\sigma}{7}$ history), disease variables (duration of pulmonary symptoms, and $\varrho$ type of SSc), and full lung function were collected at the same time as the DE. Multiple linear logistic regression was used to model the relationship between these variables and the presence of PHT as determined by DE. After matching for other indices transfer factor expressed as the percentage of predicted normal, corrected for $\mathrm{Hb}$ (TLCO) and the alveolar to arterial oxygen gradient (Aa) were the only significant independent variables for the prediction of PHT ( $p$ values of 0.0014 and 0.022 , respectively). A logistic regression equa- of tion with presence or absence of PHT as the dependent variable and if independent variables of age, sex, smoking history (ever $v$ never if smokers), SSc type, duration of pulmonary symptoms, TLCo and Aa, $\omega$ correctly designated $91.2 \%$ of cases. Twenty one of 29 patients with PHT were correctly identified $(72 \cdot 4 \%)$, whilst 82 of 84 patients 윽 without PHT were correctly assigned (97.6\%). Using TLCo and Aa alone as the independent variables, $89.4 \%$ of cases were correctly $\frac{D}{2}$ assigned using the equation:

probability $=1 / 1+[\exp \{-(1 \cdot 187-0.0867 \times \mathrm{TLCO}+0.604 \times \mathrm{Aa})\}]$

with a probability value of $\geqslant 0.5$ representing PHT. We conclude that in patients with FASSc, both TLCo and Aa gradient for oxygen are independently predictive of PHT. TLCo and arterial blood gases should be performed as part of routine assessment of patients with FASSc, and can predict patients with a high likelihood of having PHT. [Supported by a grant from the Raynauds and Scleroderma Association UK.]

Alterations in CT parenchymal density gradients in lone pulmonary hypertension complicating systemic sclerosis

JB CAILES, RM DUBOIS, DM HANSELl Royal Brompton Hospital, London Lone pulmonary hypertension (PHT) occurring in the absence of fibrosing alveolitis (FA) is a rare but well recognised complication of systemic sclerosis (SSc) which carries a poor survival o prognosis. Histologically there is fibrointimal proliferation and obliteration of the pulmonary microvasculature. Disturbances of the 0 pulmonary microvasculature can be reflected on CT scan as regional density differences of the pulmonary parenchyma. The aim of this $\frac{7}{0}$ study was to determine whether there were significant differences in CT assessed density gradients in PHT. Fifteen SSc patients, average $\mathrm{N}$ age 51.6 years ( 11 women) with depressed gas transfer (TLCo $\leqslant 80 \%$ predicted) but with no clinical, functional (forced vital capacity $97 \%$ N predicted, range 75-125) or high resolution CT evidence of fibrosing $\mathrm{\omega}$ alveolitis (FA) were studied. $3 / 10 \mathrm{~mm}$ scans were performed in the prone position. Three circular $(20 \mathrm{~mm}$ diameter $)$ regions of interest (ROI) were drawn in the most dependent (DL) and non-dependent (NDL) zone of each lung in the upper and lower lobes. Density measures (Hounsfield units - HU) were obtained for each ROI. Average DL and NDL densities were calculated in both lobes, and $\bar{O}$ global lung density measurement derived. The density gradient was $\mathbb{D}$ calculated as the difference in average density measures (DL $v$ NDL) and was assessed in the upper and lower lobes, and as a global lung measure. PHT was defined as an estimated pulmonary artery systolic pressure of greater than $30 \mathrm{~mm} \mathrm{Hg}$ at Doppler echocardiography. Five of 15 patients had evidence of PHT on echocardiography whilst 10 음 did not (NPHT). The lung density gradient was significantly smaller in the PHT group in the lower lobes decreasing by one third from median (range) $46(20-72) \mathrm{HU}$ in the NPHT group to $32 ?$ $(-9-34) \mathrm{HU}$ in the PHT group $(\mathrm{p}=0.05)$. Similar changes were noted as a global lung measure $(p=0 \cdot 05)$. We conclude that in patients with lone PHT complicating SSc there is a significant diminution of the parenchymal density gradient between dependent and nondependent lung. Determination of CT density gradients may help in the identification of patients with lone PHT. [Supported by a grant from the Raynauds and Scleroderma Association UK.] 
A prospective audit of the inpatient management of patients with chronic airflow obstruction

RM ANGUS, RKC KEITH, JW KAY, RD MONIE, NC THOMSON, KR PATEL Department of Respiratory Medicine, Western Infirmary/Gartnavel General Hospital and Southern General Hospital, Glasgow In a prospective audit we have examined the care of patients admitted with chronic obstructive pulmonary disease (COPD) to the three major hospitals serving the West of Glasgow. One in two admissions between May 1992 and 1993 were audited, evaluable data being obtained in 261 patients. The main outcome measurements consisted of the use of routine respiratory investigations and standard therapies, the length of stay, inpatient deaths, follow up and readmission rates. Home visits were also performed at two weeks and three months from discharge to assess quality of life using the hospital anxiety and depression (HAD) score and the St George's Respiratory Questionnaire. Lung function was assessed using spirometry. The mean (SD) age of patients admitted was $70 \cdot 1(8 \cdot 8), 89 \%$ were smokers with $122(47 \%)$ being male. Arterial blood gases were checked on admission in $242(93 \%)$ of patients; mean (SD) $\mathrm{PaO}_{2} 9.3(4 \cdot 6) \mathrm{kPa}, \mathrm{PaCO}_{2} 6.3(2 \cdot 0) \mathrm{kPa}, \mathrm{H}^{+}$ $41 \cdot 1(8 \cdot 2)$. One hundred and ten $(42 \%)$ had a $\mathrm{PaO}_{2}$ of $<8 \mathrm{kPa} ; 97 \%$ had a chest radiograph, $70 \%$ current or previous spirometry and $47 \%$ peak expiratory flow rates measured. Ninety five percent of patients received $\beta_{2}$ agonists, $83 \%$ anticholinergics, $31 \%$ theophyllines, $63 \%$ corticosteroids, $75 \%$ oxygen and $81 \%$ antibiotics. Doxapram was used in $7 \%$ of patients and 11 patients were given ventilatory support, nine receiving nasal ventilation and two being intubated. The mean (SD) length of stay was 11 (8.5) days with an acute mortality of $17 \%$. Sixty one percent of patients were offered outpatient appointments. Home visit $\mathrm{HAD}$ scores indicated significant anxiety and depression, mean (SD) score being $19 \cdot 8(7 \cdot 4)$ and $18 \cdot 8(7 \cdot 4)$ at two weeks and three months respectively (NS). Total St George's scores revealed considerable disability with $60(14) \%$ and $60(13 \cdot 1) \%$ respectively (NS). Spirometry was poor, mean (SD) $\mathrm{FEV}_{1}(\mathrm{l})$ of $0.77(0.40)$ and $0.74(0.34)$ at two weeks and three months respectively (NS). By 90 days there were a further 26 deaths giving an overall three month mortality of $27 \%$. Readmission rates were high with $34 \%$ being readmitted by 90 days. These findings highlight the need for new strategies to reduce readmission rates and to improve the quality of life of these patients.

\section{Explanation to patients with COPD improves quality of life after discharge}

LM OSMAN, A ENGLAND, JAR FRIEND, JS LEGGE, D GODDEN, JG DOUglas Thoracic Medicine Unit, City Hospital, Aberdeen Four hundred and seventy nine patients ranging in age from 44 years to 92 years admitted with COPD to chest and general wards were followed up by mail questionnaire within two weeks of discharge. At follow up patients were asked if their hospital admission had been worthwhile (93\% agreed) and if their chest was better than before their admission (75\% agreed). Patients were also asked if they thought their chest problem had been explained to them while they were in hospital; $74 \%$ (225) thought they had had a good or adequate explanation, $13 \%$ (39) had an explanation but had not understood it, and $13 \%$ (38) had not had an explanation. Older patients were less likely to have had an explanation $(p<0.01)$. Patients who were satisfied their problem had been explained were more likely to say their chest was better at follow up (79\%). Of those with no explanation, $68 \%$ thought their chest was better at follow up. With a poor explanation $62 \%$ thought their chest was better $(p=0 \cdot 04)$. A visual analogue scale from 1 to 10 where 1 was "not breathless at all" and 10 was "extremely breathless" was used to measure breathlessness during admission (mean (SE) $5.0(0.2)$ ) and at follow up $(5.7(0.2), \mathrm{p}=0.02)$. Controlling for age, patients with an adequate explanation had better visual analogue breathlessness scores at follow up than those who had not understood or had no explanation $(5.3 v 5.7,6.3)(p=0.05)$. Good explanation to patients is associated with well being and smaller deterioration in perceived breathlessness after discharge.

Twelve months follow up of autonomic neuropathy in patients with severe COPD

W BIERNACKI, S SCHOLEY, G BOAR, MD PEAKE Chest Unit, General Infirmary, Pontefract, West Yorkshire We have shown that subclinical autonomic neuropathy is common in patients with severe COPD
(Biernacki et al. Am $\mathcal{F}$ Respir Crit Care Med 1994;149:A1012). In one study in diabetic patients deterioration or lack of improvement of autonomic function was demonstrated over a 1.5-3 year period of observation (Ewing et al. $Q \mathcal{F}$ Med 1980;193:95). There are no published data on the natural history of autonomic neuropathy in patients with COPD. We have studied a group of 23 patients $(11 \mathrm{M})$ aged 67 (7) years with severe COPD, FEV 31 (9)\% predicted, and $\mathrm{PaO}_{2} 56$ (9) $\mathrm{mm} \mathrm{Hg}, 13$ of whom were on LTOT. We measured full standard respiratory function, blood gases and autonomic nerve function (Ewing and Clark) at entry and after one year. In $57 \%$ of patients their neuropathy score had deteriorated, in $30 \%$ there was no change, and in $13 \%$ there was slight improvement. There was no correlation between change of neuropathy score and either change in $\mathrm{FEV}_{1}$ or $\mathrm{PaO}_{2}$ over the year of study. We concluded that autonomic neuropathy deteriorates in the majority of patients with severe COPD, even those receiving LTOT.

\section{Review of the prescription of cylinder oxygen in Scotland}

E SKWARSKI, K SKWARSKI, W MACNEE Respiratory Medicine Unit, Department of Medicine, Royal Infirmary, Edinburgh; Inveresk Clinical Research, Edinburgh Long term oxygen therapy (LTOT) can be prescribed in the form of oxygen concentrators or oxygen cylinders. In Scotland concentrators can only be prescribed by chest physicians according to standard guidelines. Cylinder oxygen, which is mainly prescribed by GPs, is more costly and no guidelines have been established. The aim of this study was to determine the characteristics of patients prescribed cylinder oxygen throughout Scotland. From information given by Common Services Agency 791 practices in Scotland were contacted. The response rate was $57 \%$ giving information on 746 patients (407 M; mean age 69, range 1-99 years). COPD was the diagnosis in $74 \%$ of patients. Oxygen was prescribed for use as required in $82 \%$ of patients; on average 8 (range 1-120) cylinders/month were prescribed; $11 \%$ of patients used $>20$ cylinders/ month; $27 \%$ smoked actively at the time when oxygen was prescribed. Ninety patients (age 69 (range 36-91) years, 46 M) were visited at home after written consent was obtained from the patient's GP. In $80 \%$ of cases COPD and gradual worsening of dyspnoea was the main reason for which oxygen was prescribed; $40 \%$ smoked at the time oxygen was prescribed and $70 \%$ had been advised to use oxygen when required. They used a mean of 13 (range 1-120) cylinders/ month. Their $\mathrm{FEV}_{1}$ was $0.75(0.4) 1$, FVC $1.5(0.6) 1$. A reversibility test (to $5 \mathrm{mg}$ nebulised terbutaline) was performed at home and showed a $15 \%$ improvement in $\mathrm{FEV}_{1}$ and an absolute improvement of $200 \mathrm{ml}$ in $16 \%$ of patients. $\mathrm{SaO}_{2}$ on air, measured by portable pulse oximeter, was 90 (11)\%. During daily activities usually causing breathlessness, $\mathrm{SaO}_{2}$ fell to $88(6) \%(\mathrm{p}<0.001) ; 47 \%$ of these patients desaturated by $>5 \%$ from the baseline during exercise at home. In $1993 £ 2$ million was spent on cylinder oxygen in Scotland in contrast to $£ 350000$ on the oxygen concentrator service. This review highlights the need for guidelines for the prescription of cylinder oxygen. [Supported by Scottish Home and Health Department.]

Early effect of long term oxygen therapy on quality of life in patients with COPD

aA okubadejo, Pw jones, Ja Wedzicha Department of Thoracic Medicine, London Chest Hospital, London; Division of Physiological Medicine, St George's Hospital, London Long term oxygen therapy (LTOT) has important physiological benefits in patients with COPD and severe hypoxaemia. These patients have impaired quality of life, but it is not known whether there are any early effects of LTOT on life quality. We studied 23 patents $(15 \mathrm{~F})$ of median age 71 years with COPD and hypoxaemia, with mean (SD) $\mathrm{PaO}_{2} 6.95(0.75) \mathrm{kPa}_{1} \mathrm{PaCO}_{2} 6.52$ $(1 \cdot 22) \mathrm{kPa}, \mathrm{FEV}_{1} 0.75(0.22) 1$. They were assessed on two occasions two weeks apart in the month before starting LTOT, and then a third time two weeks after starting LTOT. We selected a control group of 18 patients $(6 \mathrm{~F})$ with $\mathrm{COPD}$ and $\mathrm{FEV}_{1}<1 \cdot 51$, but without severe hypoxaemia. Their median age was 72 years, mean (SD) $\mathrm{PaO}_{2} 8 \cdot 17$ $(0.90) \mathrm{kPa}, \mathrm{PaCO}_{2} 6.02(0.75) \mathrm{kPa}, \mathrm{FEV}_{1}$ 0.94 (0.331. The control group had three assessments using the same time intervals, but were not started on LTOT. At each assessment both groups underwent spirometry, blood gas analysis, and quality of life assessment using the Sickness Impact Profile (SIP), the St George's Respiratory Questionnaire (SGRQ), and the Hospital Anxiety and Depression Scale (HAD). Before starting LTOT the subjects had a higher mean SGRQ 
total score than the controls $(61.9 v 47.0, \mathrm{p}=0.007)$, implying a greater impairment in life quality. There were no significant differences between the two baseline assessments in mean score for any measured parameter for either group. At the third assessment after two weeks the mean SGRQ score of the 23 patients fell from 61.1 to 55.0 ( $p=$ 0.045 ) suggesting improved life quality; however, the control group also experienced a fall (from 45.8 to $41 \cdot 8$ ) and the mean change was not significantly different between groups $(p=0.6)$. Changes in anxiety, depression and SIP scores were not significantly different between groups. Although we have demonstrated an improvement in the quality of life of patients after just two weeks of oxygen therapy, this was not significantly greater than that shown by a group of patients without severe hypoxaemia who were not treated with oxygen.

Subjective reactions of patients and their carers to liquid oxygen

ME HYLAND, I COUTTS, M WINTERTON Department of Psychology, University of Plymouth; Treliske Hospital, Cornwall The aim was to investigate the subjective benefits of a portable liquid oxygen (LOX) delivery system for patients and their carers. Patients were included if they satisfied an economic criterion of using two or more cylinders of oxygen per week and were considered likely to benefit from LOX. Patients were considered suitable if their oxygen saturation fell during a six minute walk and if in the clinician's view they were motivated to increase activity. One patient declined to use LOX. Of those 44 patients using LOX (22 patients having COPD, 10 having pulmonary fibrosis, and a mixed group of 12 patients with other diseases), 33 completed quality of life (QOL) records over a period of four months or more. QOL was measured by a specially constructed questionnaire: an activity restriction checklist (including space for other activities to be listed) and a scale measuring the perceived impact of LOX. The activity checklist was administered before and after treatment and the overall evaluation was made monthly after treatment. The mean number of activities restricted prior to LOX was 13.9 (maximum 23, minimum 7), and mean number regained after one month $=5 \cdot 6$ (maximum 17, minimum 1). All patients reported that LOX made their life better, but to different degrees, and all but one carer reported that the carer's life had also improved. Twenty seven carers felt that LOX had made the patient less dependent, three felt it made no difference, and three felt that the patient had become more dependent on the carer. Patient's and carers' evaluations of life improvement were significantly $(\mathrm{p}<0.001)$ correlated $(r=0.64)$, but patients' evaluations of life improvement were not significantly related to carers' evaluations of dependency $(r=0 \cdot 21)$. There was a significant improvement in evaluation between the first and second month of LOX use, suggesting that there is a learning component to the advantages gained from LOX.

Factors predicting subjective benefit of patients prescribed liquid oxygen: a pilot study

I COUTTS, M WINTERTON, ME HYLAND Treliske Hospital, Cornwall; Department of Psychology, University of Plymouth The purpose of this study was to investigate whether physiological variables measured prior to the introduction of liquid oxygen (LOX) predicted the degree of subjective benefit after two months treatment with LOX. Thirty one patients were prescribed LOX on the basis of cylinder use (two or more cylinders used per week), oxygen desaturation during the six minute walk, and the clinician's opinion that the patient was motivated to increase activity. For all 31 patients $\mathrm{FEV}_{1}$, FVC, TLC, TLCO, Kco, six minute walk distance with oximetry, and resting blood gases were measured pretreatment; the average of patients' overall evaluation of LOX using a seven-point evaluation scale measured at one and two months after treatment was the outcome variable. Mean (SD) values of physiological variables were: $\mathrm{FEV}_{1}=0.97(0.64) 1$, \% predicted $\mathrm{FEV}_{1}=$ $36, \mathrm{FVC}=1 \cdot 71(0.81) 1$, \%predicted $\mathrm{FVC}=50$, mean resting $\mathrm{PCO}_{2}=$ $5.4(0.98) \mathrm{kPa}$, mean resting $\mathrm{Po}_{2}=8.7(1.24) \mathrm{kPa}, \%$ predicted $\mathrm{KCO}=$ $63, \%$ predicted TLC $=84, \%$ predicted TLCO $=43$, pre-exercise oxygen saturation $=93 \cdot 2 \%$, postexercise oxygen saturation $=86 \%$, mean distance walked $=223.4 \mathrm{~m}$. There was a significant negative correlation between improvement and pretreatment resting $\mathrm{PCO}_{2}(r=-0.41, \mathrm{p}=$ $0.03)$. There were no other significant correlations; improvement was not significantly related to oxygen desaturation during exercise $(r=$ $0 \cdot 16)$. These data suggest that it may be difficult to predict which patients will report most subjective benefit from LOX on the basis of physiological data alone.

\section{Evaluation of the use of concentrators for domiciliary oxygen} supply for less than eight hours per day

M JACKSON, JM SHNEERSON Respiratory Support and Sleep Centre, Papworth Hospital, Papworth Everard, Cambridge Since their introduction in 1985, oxygen concentrators have only been recommended when domiciliary oxygen is used for over eight hours per day. Subsequent changes in the prices of oxygen merit a reappraisal of the prescribing of concentrators and cylinders when oxygen is used for less than eight hours per day. Twenty six patients in two health districts who used oxygen for less than eight hours per day completed $\stackrel{\oplus}{\rightarrow}$ a crossover study in which each group received oxygen from each source for consecutive three month periods. The patients were visited $\overline{\bar{\sigma}}$ at home before and during the study and on each visit they completed $\frac{\bar{\sigma}}{\sigma}$ a questionnaire asking about their use of oxygen, how acceptable they found the two sources, and about several dimensions of their quality of life. The theoretical minimum cost of cylinder supply, the actual ${ }^{\text {o }}$ cost of cylinder supply, and the average concentrator costs were $\overrightarrow{0}$ assessed. The patients found the concentrators to be more acceptable, $\overrightarrow{\vec{\omega}}$ more useful, and less obtrusive than cylinders. They used more oxygen $\vec{\omega}$ in more rooms of the home during treatment with concentrators and there were improvements in the quality of life measurements. The costing information showed that, both in theory and in practice, of oxygen concentrators are cheaper than cylinders when more than about 1.4 hours per day of oxygen are used. These results suggest that the provisions for the supply of domiciliary oxygen should be reviewed and that concentrators should be considered for patients $\overrightarrow{0}$ who use more than around 1.4 hours per day.

Use of oxygen concentrators along with nebulisers in the Birmingham and Solihull areas: who are using them and why?

J AYRES, P OTTAWAY Chest Research Institute, Birmingham Heartlands Hospital; DeVilbiss Health Care Many patients who use an oxygen concentrator may also be using nebulisers. We set out to determine how many patients were using both nebulisers and oxygen concentrators to assess whether there would be a benefit in trying to $\mathbb{D}$ develop a combined concentrator and nebuliser. In May 1994320 @ patients in the Birmingham and Solihull areas who were using oxygen $\overrightarrow{\overline{0}}$ concentrators were sent a questionnaire asking for information on 3 diagnosis, nebuliser use, and other factors concerning the machines and their use. $224(70 \%)$ of questionnaires were returned after aO single mailing. Nine patients had died, three forms were inadequately completed, and one patient had moved, leaving 211 for analysis. 0 Eight were being used by children and these are excluded from the following analysis. The mean age (range) of the remaining 203 patients was $65.5(21-88)$ years. Eighty four (41\%) were women. $148(73 \%) 3$ of patients had COPD or an equivalent diagnosis and $20(10 \%)$ hadi a diagnosis of asthma. Thirty $(15 \%)$ admitted to being a current $\frac{0}{3}$ smoker. One hundred and five $(51 \%)$ had a nebuliser at home which they used at least once a day. Four of the nebuliser group had a "non-음 airways disease" reason for having a concentrator compared with 24 D of 98 in the non-nebuliser group, five of whom gave as their reason을 "heart failure". We did not enquire as to how many had had formal $N$ blood gas assessment. Problems identified by the patients with respect to nebulisers were noise $(29$ of $105,28 \%)$ and time taken $(10$ of $105, N$ $9 \cdot 5 \%$ ), while 45 of $203(22 \cdot 2 \%)$ complained of concentrator noise.N If these figures can be applied to the rest of the West Midlands, over 500 patients would be candidates for a combined concentrator $K$ nebuliser. Assessment of a prototype combined machine is underway. 电

Home nebulisers: can optimal treatment be predicted by responses to St George's Respiratory Questionnaire?

M ZAMAN, M ALLEN, MF MUERS Respiratory Unit, Killingbeck Hospital,o Leeds Domiciliary nebulisers are in widespread use for the treatment of patients with severe chronic asthma or chronic obstructive pul-o monary disease (COPD), to relieve symptoms and improve their quality of life. It has been suggested that a $15 \%$ improvement in home peak expiratory flow rate (PEFR) along with a subjective? preference for nebuliser treatment after a domiciliary trial can identify patients who should receive long term nebuliser treatment. We tested the hypothesis that this combination of objective and subjective benefit would be reflected in a more formal measure of respiratory related quality of life. We recorded patients' responses to the St George's Respiratory Questionnaire (SGRQ) in our nebuliser trials at baseline (Q1), at six weeks (at trial end, Q2) and after 12-16 weeks (Q3). We 
studied 29 patients $(16 \mathrm{M})$ of mean $(\mathrm{SD})$ age $66 \cdot 2(5 \cdot 3), \%$ predicted FEV $_{1} 39 \cdot 4$ (17.9), \% predicted PEFR 40.3 (15.0). Twelve patients were "responders" with a $>15 \%$ improvement in home PEFR 26.6 (12)\%, and with personal preferences; 17 were "non-responders" with PEFR improvement of $1.8(14.0) \%$. No significant differences were observed. For Q1-Q2 the mean total SGRQ change for responders was $-0.83(95 \% \mathrm{CI}-7 \cdot 2$ to 6.5$)$, for non-responders $-0.47(95 \% \mathrm{CI}-7 \cdot 3$ to $6 \cdot 6) ; \mathrm{p}=0.9$. For $\mathrm{Q} 1-\mathrm{Q} 3$ the mean change for responders was $+2 \cdot 8(95 \% \mathrm{CI}-5 \cdot 9$ to $7 \cdot 2)$ and non-responders $+2 \cdot 15(95 \%$ CI $-6 \cdot 3$ to $7 \cdot 5), p=0 \cdot 39$. The lack of correlation between nebuliser treatment benefit is measured by SGRQ and by the combination of peak expiratory flow rate change and subjective preference needs further investigation.

\section{Lack of association between HLA-DPB and sarcoidosis}

PA LYMPANY, AM SOUTHCOTT, KI WELSH, RM DU BOIS Interstitial Lung Disease Unit, Royal Brompton Hospital, London Sarcoidosis is a chronic granulomatous disorder which is characterised by the accumulation of activated CD4 $+\mathrm{T}$ lymphocytes at the sites of disease. It has been established that $\mathrm{CD} 4+\mathrm{T}$ cells are activated when presented with an antigen in the context of cell surface molecules encoded by the major histocompatibility complex (MHC) and that certain MHC molecules are associated with sarcoidosis. Recent studies of chronic beryllium disease have identified an association between this disease and HLA-DPB alleles, which are encoded by MHC class II genes (Richeldi et al. Science 1993;262:242-4). We wished to show whether this association is specific for chronic beryllium disease or is a general marker of granulomatous disease. The aims of this study were therefore to define associated HLA-DP alleles in sarcoidosis and to compare the frequency of HLA-DP alleles in two groups of subjects; 49 subjects with sarcoidosis and 76 unselected normal subjects. The second exon of the HLA class II DPB1 chain has been shown to exhibit extensive polymorphism and in this study, the polymerase chain reaction was used to amplify the second exon of HLA-DPB1 in each subject. Subsequently hybridisation with sequence specific oligonucleotide probes was used to identify specific HLA-DPB1 alleles. It was possible to identify 19 HLA-DPB1 alleles using this technique. There were no significant differences in the phenotype frequency of any HLADPB allele when compared with the normal population ( $p>0.5$ in all cases) when the significance values were corrected for the number of comparisons made. We therefore conclude that there is no significant association between specific HLA-DPB alleles and sarcoidosis and that the HLA-DPB association previously demonstrated in chronic beryllium disease is not a general marker for granulomatous diseases.

Tumour necrosis factor alpha (TNF $\alpha$ ) regulation by interleukin 10 (IL-10) in alveolar macrophages from patients with sarcoidosis (S) or fibrosing alveolitis (CFA)

L ARMSTRONG, AB MILlaR School of Postgraduate Medicine, University of Bath, Bath We are interested in the regulation of the pulmonary inflammatory response, in particular the regulation of TNF $\alpha$ by IL10. Abnormalities of these regulatory mechanisms have been proposed to occur in sarcoidosis (S) and CFA. In a previous study ( $A m$ Rev Respir Dis 1994;149:A1066) we demonstrated that IL-10 is a potent inhibitor of TNF $\propto$ production by normal human alveolar macrophages (AM) and peripheral blood monocytes (PBM). We hypothesised that in AMs from S or CFA, IL-10 inhibition of TNF $\alpha$ production may be deficient. Bronchoalveolar lavage and venepuncture were performed on all subjects. Recovered AM and PBM were cultured for a period of 24 hours in the presence of $10 \mu \mathrm{g} / \mathrm{ml}$ lipopolysaccharide (LPS) alone and with IL-10 (DNAX) at a dose range 0.1-100 $\mathrm{U} / \mathrm{ml}$. The free unbound TNF $\alpha$ levels in the AM supernatants were determined by ELISA. There were no significant differences between basal or LPS-induced AM TNF $\alpha$ levels from normals, CFA or S, although the LPS-induced values were lower for S $(1.479(0.824))$ than for normal subjects $(3.508(0 \cdot 629), p=0.071)$. In normal subjects $(\mathrm{n}=6)$ LPS-induced TNF $\alpha$ levels were significantly reduced in comparison to control values $(p<0.01)$ by a minimum dose of $50 \mathrm{U} / \mathrm{ml}$ IL-10, whereas LPS-induced TNF $\alpha$ levels from sarcoid AM $(n=12)$ were not significantly affected by IL-10 at any dose. In contrast LPSinduced TNF $\alpha$ levels from CFA $(n=10)$ were significantly reduced by a minimum dose of $0 \cdot 1 \mathrm{U} / \mathrm{ml}$ IL-10. IL-10 inhibition of LPSinduced TNF $\alpha$ from PBM was similar in all three groups. These results suggest an enhanced sensitivity of CFA AM to IL-10, contrasting with the apparent insensitivity of sarcoid AM, either to LPS induction of TNF $\alpha$ or reduction by IL-10. Thus, IL-10 may have a complex role in the regulation of pulmonary $\mathrm{TNF} \alpha$ in the inflammatory response.

Inhibition of growth factor-stimulated tyrosine phosphorylation induces apoptosis in small cell lung cancer cell lines

A TALLETT, T SETHI Respiratory Medicine Unit (RIE), Rayne Laboratory, University of Edinburgh Medical School, Edinburgh Small cell lung cancer (SCLC) constitutes $25 \%$ of all lung cancers and follows an aggressive clinical course. Novel approaches to treatment are needed. It is likely that these will arise from a better understanding of the intracellular molecular events underlying mitogenesis. SCLC cells secrete many hormones and neuropeptides. We have previously shown that multiple neuropeptides induce inositol phosphate hydrolysis, mobilisation of intracellular calcium and stimulate growth, suggesting that SCLC growth is mediated by an extensive network of autocrine and paracrine interactions. There is increasing interest in protein tyrosine phosphorylation as a growth promoting signal and we therefore studied the effects of serum, insulin, bombesin and gastrin, all of which are well recognised growth factors, on tyrosine phosphorylation in SCLC. Following stimulation with growth factors, whole cell lysates were analysed by western blotting. We demonstrated that these growth factors were all able to induce protein tyrosine phosphorylation in SCLC in a concentration and time-dependent fashion. An increase in tyrosine phosphorylation was evident at 30 seconds increasing to a maximum at 10-20 minutes and then declining. Though the pattern of tyrosine phosphorylation was agonist specific, some major substrates like $\mathrm{p} 125^{\mathrm{FAK}}$ and $s r c$-related kinases were phosphorylated by all growth factors. Tyrphostin 25 , a specific tyrosine kinase inhibitor, inhibits this tyrosine phosphorylation, SCLC growth and stimulates apoptosis in the $\mu \mathrm{M}$ range $\left(\mathrm{IC}_{50} 10 \mu \mathrm{M}\right)$. Increased rate of apoptosis compared to controls was evident at between 8 and 12 hours and was maximal at 48-72 hours, demonstrated morphologically and by DNA fragmentation analysis (table,

\begin{tabular}{lllllll}
\hline & \multicolumn{6}{l}{ Time (hours) } \\
\cline { 2 - 7 } & 3 & 8 & 12 & 24 & 48 & 72 \\
\hline Apoptosis (\%) & $3(2)$ & $5(4)$ & $7(3)$ & $15(5)$ & $30(8)$ & $35(7)$ \\
\hline
\end{tabular}

$\mathrm{n}=4$, mean (SE)). These results indicate that tyrosine phosphorylation is an important mitogenic signal for small cell lung cancer. Tyrosine phosphorylation is also an important growth signal for nonSCLC, hence inhibition of this pathway may inhibit the growth of all histological types of lung cancer and be an important target for novel therapeutic strategies. [This work has been supported by the British Lung Foundation.]

Raised interleukin 10 (IL-10) levels in bronchoalveolar lavage (BAL) samples in the adult respiratory distress syndrome (ARDS)

SC DONNELLY, PT REID, RM STRIETER, SK KUNKEL, M BURDICK, I ARMSTRONG, IS GRANT, C HASLETT Respiratory Medicine Unit, University of Edinburgh; Pulmonary and Pathology, University of Michigan; Intensive Therapy Units, Royal Infirmary and Western General Hospitals, Edinburgh ARDS is a catastrophic form of acute inflammatory lung injury. IL-10 plays an important immunomodulatory role in macrophage function. It is a potent inhibitor of the secretion of the proinflammatory cytokines (eg IL-1 $\beta$, TNF $\alpha$ ) from monocytes/macrophages while at the same time stimulating the release of mediators with anti-inflammatory activity (eg IL-1 ra). In this study we investigated whether the level of IL-10 within the alveolar airspaces was of prognostic significance. BAL sampling was performed on established ARDS patients within 24 hours of diagnosis. Patient group data are expressed as median values and analysed using Mann-Whitney. IL10 assays were performed using a sandwich ELISA. Twenty seven ARDS patients (9/27 died) and a ventilated trauma control group of nine (4/9 died) were enrolled. IL-10 values for patient groups are summarised in the table. BAL IL-10 values were significantly raised 


\begin{tabular}{lllll}
\hline & $\begin{array}{l}\text { ARDS } \\
(n=27)\end{array}$ & $\begin{array}{l}\text { Control } \\
(n=9)\end{array}$ & $\begin{array}{l}\text { ARDS } \\
\text { survivors } \\
(n=18)\end{array}$ & $\begin{array}{l}\text { ARDS non- } \\
\text { survivors } \\
(n=9)\end{array}$ \\
\hline $\mathrm{IL}-10$ & $0 \cdot 10$ & $0 \cdot 00$ & $0 \cdot 115$ & $0 \cdot 05$ \\
$(\mathrm{ng} / \mathrm{ml})$ & $(0-1 \cdot 60)$ & $(0-0 \cdot 052)^{* * *}$ & $(0 \cdot 03-1 \cdot 60)$ & \begin{tabular}{l}
$(0-0 \cdot 113)^{* *}$ \\
\hline
\end{tabular}
\end{tabular}

${ }^{* *} \mathrm{p}<0 \cdot 01 ;{ }^{* * *} \mathrm{p}<0.0005$.

in ARDS patients compared with controls $(p=0.0005)$. In addition, low values correlated significantly with patient mortality $(p<0.01)$ and a lower $\mathrm{PaO}_{2} / \mathrm{FIO}_{2}(\mathrm{p}<0.05)$. Thus, significantly raised levels of the anti-inflammatory cytokine IL-10 were found in BAL samples from ARDS patients. In addition, high IL-10 levels correlated with patient survival. Our data support the hypothesis that an inability to mount a significant intrapulmonary localised anti-inflammatory cytokine response contributes towards a worse overall patient prognosis.

Plasma thrombomodulin ( $\mathrm{Tm}$ ) in patients "at risk" and with established adult respiratory distress syndrome (ARDS)

PT REID, SC DONNELLY, L BRUCE, AM PERRIE, CR ROBERTSON, TS GRANT, DC CARTER, IR MACGREGOR, C HASLETT Respiratory Medicine Unit, Departments of Surgery, Accident $\mathcal{E}$ Emergency, Intensive Therapy Unit; Scottish National Blood Transfusion Service, Edinburgh ARDS represents the pulmonary component of an acute inflammatory microvascular injury in response to a variety of disparate aetiological conditions. Tm is an endothelial surface glycoprotein which may be cleaved and exist in the circulation in a soluble form (sTm). Raised levels have been described in a variety of disease states characterised by damage to the vascular endothelium. In this study we investigated whether significant circulating levels of $\mathrm{sTm}$ existed in established ARDS and whether levels in the "at-risk" period had any predictive role for ARDS. Fifty eight "at risk" patients, 16 with established ARDS and a ventilated control group of eight patients were enrolled. Blood sampling was performed on initial hospital presentation for "at risk" and within 24 hours of diagnosis for ARDS patients. sTm was assayed by a sandwich ELISA technique. Non-parametric statistical methods were employed. Significantly higher levels of $\mathrm{sTm}$ were found in patients with established ARDS than in controls $(p=0.0001)$ or those at risk of ARDS $(p=0 \cdot 001)$. No significant difference was found in initial sTm levels between patients who progressed to ARDS compared with those who did not $(\mathrm{p}<0 \cdot 1)$. No significant association was found with subsequent mortality $(p=0 \cdot 2)$, ventilatory days $(p=$ $0 \cdot 06)$ or organ failure score $(p=0 \cdot 38)$. Group patient data are summarised in the table. Our data suggest that sTm provides a marker

\begin{tabular}{|c|c|c|c|c|}
\hline & \multirow[t]{2}{*}{ Control } & \multirow{2}{*}{$\begin{array}{l}\text { Established } \\
\text { ARDS }\end{array}$} & \multicolumn{2}{|l|}{ At risk } \\
\hline & & & $\begin{array}{l}\text { Non-ARDS } \\
\text { progression }\end{array}$ & $\begin{array}{l}\text { ARDS } \\
\text { progression }\end{array}$ \\
\hline $\begin{array}{l}\text { sTM } \\
(\mu \mathrm{g} / \mathrm{ml})\end{array}$ & $\begin{array}{l}25 \cdot 4 \\
(4 \cdot 3-55 \cdot 0)\end{array}$ & $\begin{array}{l}106 \cdot 9 \\
(50 \cdot 1-346 \cdot 8)^{* * *}\end{array}$ & $\begin{array}{l}26 \cdot 7 \\
(4 \cdot 4-115 \cdot 3)\end{array}$ & $\begin{array}{l}68 \cdot 5 \\
(4 \cdot 0-121 \cdot 0)(N S)\end{array}$ \\
\hline
\end{tabular}

*** $\mathrm{p}=0.001 ; \mathrm{NS}=$ not significant.

of endothelial damage in established ARDS but does not provide a marker of impending ARDS in the initial blood sample from patients "at risk" of ARDS.

\section{Lung albumin distribution and content in normobaric and} hyperbaric hyperoxia in the rat

PW JOHnston, ST NG, AD MCKInNon, J woo, JAS ROSS Departments of Pathology and Environmental and Occupational Medicine, University of Aberdeen, Foresterhill, Aberdeen In oxidative lung injury pulmonary oedema results from increased permeability. To assess the severity of injury and its relation to pressure, it would be useful to measure lung leak of a marker. Four groups $(n=8)$ of rats were exposed to $21 \mathrm{kPa}$ and $100 \mathrm{kPa}$ of oxygen at two or 30 atmospheres absolute (atm abs) with equivalent decompression schedules. Using a new method of image analysis of tissue sections stained with antibody against endogenous albumin, we assessed both the distribution and amount of albumin in resin sections stained with an immunogold method for rat albumin and enhanced with silver for light microscopy. Area fraction (median and interquartile ranges) of positive albumin staining in $100 \mathrm{kPa}$ oxygen exposed groups was greater than $21 \mathrm{kPa}$ oxygen groups regardless of ambient pressure: $1 \mathrm{kPa} 2 \mathrm{~atm}$ abs, $0.65 \%(0 \cdot 60$ $0 \cdot 78 \%) ; 100 \mathrm{kPa} 2$ atm abs, $2 \cdot 85 \%(2 \cdot 28-3 \cdot 53 \%) ; 21 \mathrm{kPa} 31$ atm abs, $0.60 \%(0.33-1 \cdot 20 \%) ; 100 \mathrm{kPa} 31 \mathrm{~atm}$ abs, $2 \cdot 45 \%$, (1.93-3.05\%). Amounts of albumin, corrected for body weight, were deduced and show large increases in the hyperoxic groups, again regardless of exposure pressure: $21 \mathrm{kPa} 2 \mathrm{~atm}$ abs, $53 \cdot 2 \mu \mathrm{g} / \mathrm{g}(44 \cdot 2-60 \cdot 2 \mu \mathrm{g} / \mathrm{g})$; $100 \mathrm{kPa} 2 \mathrm{~atm}$ abs, $166 \mu \mathrm{g} / \mathrm{g}(136-172 \mu \mathrm{g} / \mathrm{g}) ; 21 \mathrm{kPa} 31 \mathrm{~atm}$ abs, $48.0 \mu \mathrm{g} / \mathrm{g} \quad(5 \cdot 2-87.7 \mu \mathrm{g} / \mathrm{g}) ; \quad 100 \mathrm{kPa} \quad 31 \mathrm{~atm} \quad$ abs, $\quad 151 \mu \mathrm{g} / \mathrm{g}$ $(126 \cdot 5-155 \cdot 0 \mu \mathrm{g} / \mathrm{g})$. Statistical analysis confirms the differences relate to partial pressure of oxygen and not exposure pressure. We suggest that risks of oxidative injury in hyperoxia result from the partial pressure of oxygen and are not pressure dependent.

\section{4-Hydroxy-2-nonenal levels in the plasma of patients with ARDS}

GJ QUINLAN, TW eVANS, JMC GUTTERIDGe Royal Brompton Hospital, London Recent findings have shown that the n-6 fatty acid oxidation product, 4-hydroxy-2-nonenal (HNE) is increased in the plasma of ARDS patients compared with healthy controls and that such increases are related to losses in the polyunsaturated fatty acid linoleic acid. We have now developed a more sensitive assay of HNE based on gas chromatography mass spectrometry of an HNE trimethylsilyl (TMS) derivative. Results are in agreement with those obtained previously. ARDS non-survivors had higher HNE levels $(0.532(0.069) \mathrm{nmol} /$ $\mathrm{ml})$ than survivors $(0.433(0.048) \mathrm{nmol} / \mathrm{ml})$, although not significantly $(p=0 \cdot 6)$. Comparison of both groups with healthy controls $(0 \cdot 205$ $(0.03) \mathrm{nmol} / \mathrm{ml})$ showed highly significant $(\mathrm{p}<0.0001)$ differences. In addition, a control group of cardiopulmonary bypass patients was also included $(0.279(0.027) \mathrm{nmol} / \mathrm{ml})$. These were significantly different from the normal control group $(p<0.03)$ and from the ARDS nonsurvivors $(p>0 \cdot 03)$, but not from survivors $(p=0 \cdot 1)$. HNE is a lipid peroxidation product with several well documented biological effects such as cytotoxicity, enzyme inhibition and chemotactic properties. Our findings show that ARDS patients are under considerable oxidative stress which results in molecular damage and the formation of toxic oxidation products including HNE. These products may influence the course of ARDS in non-surviving patients. The increased levels of HNE in patient controls compared with healthy controls is probably a reflection of their oxidative stress from ventilatory support. This new assay technique on a larger patient population is in agreement with our previous findings.

Plasma 4-hydroxy-2-nonenal levels during cardiopulmonary bypass and their relationship to the iron loading of transferrin

GJ QUINLAN, S MUMBY, J PEPPER, TW EVANS, JMC GUTTERIDGE Royal Brompton Hospital, London 4-hydroxy-2-nonenal (HNE) is an aldehydic lipid peroxidation product formed in vitro in model systems from the oxidation of n-6 fatty acids. It has also been detected and measured in vivo in plasma and tissue. HNE is a highly reactive molecule which can exert profound influences on biological systems including enzyme inhibition and chemotaxis. Reactive forms of iron have been shown to be essential catalysts for HNE formation, and plasma linoleic acid has been implicated as a substrate for its formation in patients with ARDS. We have measured HNE levels using gas chromatography mass spectrometry, in 12 cardiopulmonary bypass (CPB) patients before and after bypass. When corrected for haemodilution, by relating results to total protein levels, all bypass patients showed increases in HNE after bypass $(11.5(2 \cdot 7) \mathrm{pmol} / \mathrm{mg}$ protein) compared with levels before bypass $(8.5(2.4) \mathrm{pmol} / \mathrm{mg}$ protein) Further, when plasma catalytic iron levels were measured as bleomycin-chelatable iron, five of the 12 patients were found to be iron overloaded after bypass, and it was these patients who showed the greatest percentage increase in $\mathrm{HNE}(90 \cdot 6 \%(16 \cdot 85))$ compared with patients not iron overloaded $(21 \cdot 61 \%(5 \cdot 98))$, differences $(p=0 \cdot 01)$. These results indicate that CPB patients are under oxidant stress as a consequence of extracorporeal blood circulation, which can release catalytic iron and exacerbate oxidative damage. CPB patients are also subjected to further oxidative stress from ischaemia/reperfusion injury associated with cross clamp release, and reactive iron will increase oxidative damage. Our results support the use of chelation therapy during bypass. 
Temporal pattern of enhanced expression of procollagen-1 in tuberculin induced delayed type hypersensitivity (DTH)

Bg MARShall, A WANGoo, HT CooK, RJ SHAW Departments of Respiratory Medicine and Histopathology, St Mary's Hospital Medical School, London Tissue fibrosis is a common and serious consequence of chronic inflammation in lungs of patients with tuberculosis. Mechanisms linking these processes are poorly understood. In this study we have used the human in vivo model of the tuberculin Heaf test to define the temporal sequence of events leading to the increase in new collagen production, since this would provide important evidence that a DTH response can progress to a fibrotic reaction. Thirty six volunteers with grade 3/4 Heaf reactions (six groups of six) underwent a skin biopsy at the site of tuberculin test at intervals from day 1 to 14 . A group of 12 controls received normal saline. Immunohistochemical analysis revealed increased expression of procollagen peptide 1 (PCP1) as early as day 3 following the tuberculin Heaf test and was maximal on day 14. There was marked expression of PCP-1 in the perivascular infiltrates and in the surrounding interstitial infiltrate. These data from a human in vivo model of a delayed type hypersensitivity response

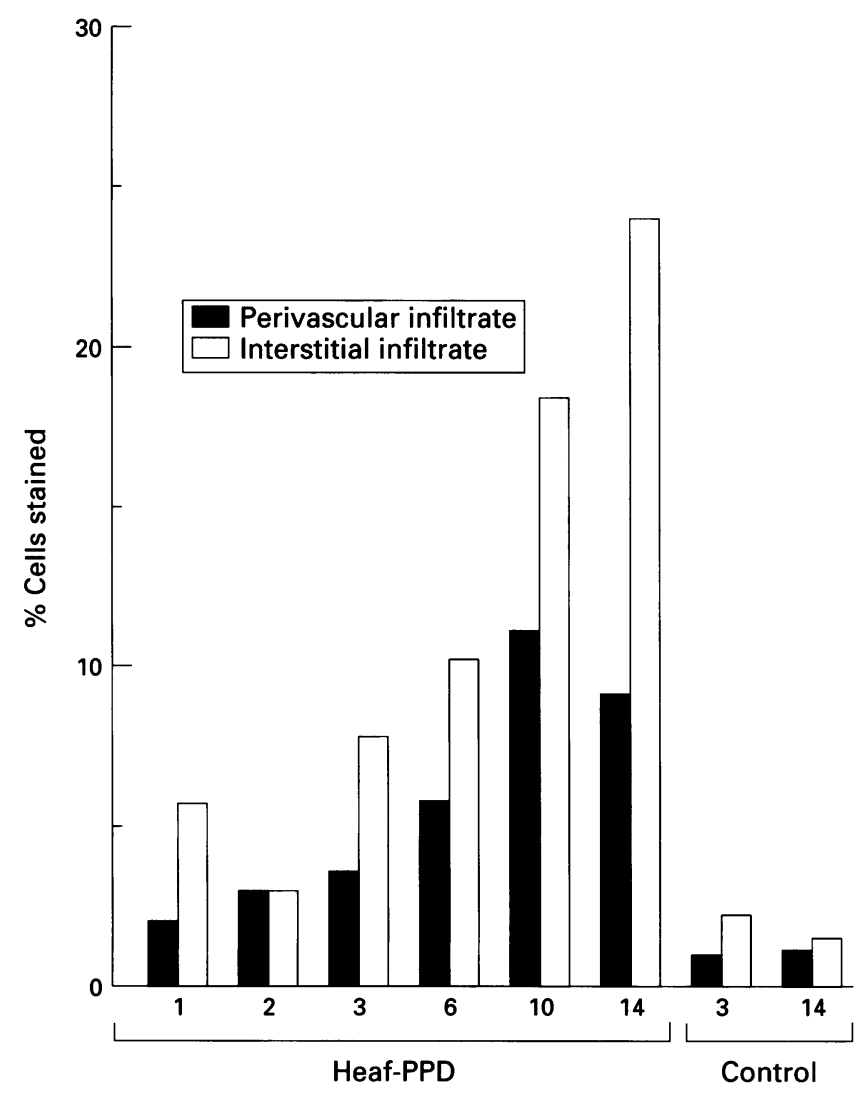

Day of biopsy post injection

provide information on the course of events in this type of chronic inflammation, and indicate that the immune response is intimately associated with the initiation of a fibrotic response.

\section{Tuberculosis among the homeless in London}

D KUMAR, K CITRON, J LEESE, J WATSON PHLS Communicable Disease Surveillance Centre (CDSC); Royal Brompton Hospital; Department of Health Tuberculosis is a well recognised problem among the homeless. Recent figures show that tuberculosis notifications are no longer declining in England and Wales, with an increase of around 5\% per year in the last two years. An increase in homelessness has been suggested as one of the possible contributors to this increase. Reliable estimates of the prevalence or incidence of tuberculosis in the homeless subgroup are not available for this country. A study was undertaken to estimate the prevalence of active pulmonary tuberculosis in a homeless population in London and to assess whether those with suspected disease could be integrated into the existing health care system for further follow up and treatment. Voluntary screening programmes based on a questionnaire survey and chest radiography were set up during Christmas 1992 and 1993 at temporary shelters for the homeless in London. In 1992 nearly 1600 people visited the centre, of whom 372 volunteered for the screening and 342 were $x$ rayed. Nineteen $(5 \cdot 4 \%)$ of those $x$ rayed had radiological features suggestive of active tuberculosis. In 1993 around 2000 homeless people visited the centre, of whom 270 volunteered for the screening and 253 were $x$ rayed. Eleven $(4 \cdot 4 \%)$ had features consistent with active tuberculosis on the basis of the chest $x$ rays and clinical examination by a chest physician. Overall, among 595 people $x$ rayed in the two surveys $30(5 \%)$ had changes suggestive of active tuberculosis. Further investigations confirmed nine $(1 \cdot 5 \%)$ with active pulmonary disease and eight with no active tuberculosis. In 13 the diagnosis was not determined as four declined further investigation and nine did not attend their hospital appointment.

Trends in tuberculosis notifications in England and Wales, 1982-93

AC HAYWARD, JM WATSON PHLS Communicable Disease Surveillance Centre, London Between 1987 and 1993 over 10000 more cases of tuberculosis (TB) were notified than would have been expected if previous trends had continued. TB notifications from 1982 to 1993 were analysed to determine the population groups and geographical areas in which increases occurred Three year average notification rates for the years 1986-8 were compared with those for 1991-3 (or 1990-2 when notification data were unavailable for 1993) in order to calculate comparative rates (CR (within 95\% confidence limits)). Increases were greatest in Regional Health Authorities (RHAs) with large urban conurbations (North East Thames: CR=1.33 (1.251.41); West Midlands (CR $=1 \cdot 12(1 \cdot 05-1 \cdot 18)$. Rates in rural RHAs generally continued to decline (Wales $C R=0.82(0.91-0.93)$; Wessex $\mathrm{CR}=0.81(0.7-0.93))$. In men significant increases were seen in those aged 15-25 years $(C R=1 \cdot 2(1 \cdot 09-1 \cdot 32)$ and decreases were seen in those over 35 years. In women no age groups experienced significant changes in rates. Non-respiratory TB accounted for $21 \cdot 2 \%$ of notifications in 1987 and $27 \%$ in 1993. In most age/sex/geographical groups significant increases were confined to non-respiratory TB. However, increases were seen in both respiratory and non-respiratory rates $(C R=1.24$ and 1.28 respectively) in men aged 15-34 years in North Thames RHA. Some of the increases observed may be due to increased reporting of TB cases following the appointment of Consultants in Communicable Disease Control. This is supported by selective increases in non-respiratory notifications which may have been subject to greater under notification (than respiratory TB) in the past. Other factors associated with recent increases will be discussed. In addition the results of the 1993 National Tuberculosis Survey, which includes information on ethnicity, will further elucidate the causes of the increases.

\section{More ambiguities and inaccuracies in the notification of cases} of tuberculosis in England and Wales

D KUMAR, J WATSON, J DARBYSHIRE ON BEHALF OF A PHLS/BTS/DH COLlaborative GROUP PHLS Communicable Disease Surveillance Centre, London; MRC HIV Clinical Trials Centre, Royal Brompton Hospital, London There has been a statutory requirement to notify all cases of tuberculosis, on clinical suspicion, since 1912. "Ambiguities and inaccuracies" in the notification of tuberculosis were identified in the $1978 / 79$ MRC Tuberculosis Notifications Survey and addressed in a Code of Practice for notification published by the British Thoracic Association in 1982. The 1993 National Tuberculosis Survey has revealed that problems in the reporting of tuberculosis, both old and new, remain. Copies of 6118 notification forms were received in the 1993 National Tuberculosis Survey of which 550 were duplicates (117 cases were reported by two different local authorities and 140 were reported twice by the same local authority). Five hundred and sixty three cases receiving chemoprophylaxis only were notified; in $\mathbf{4 2 5}$ this was stated on the notification form (and could therefore be excluded from statistics prepared by the Office of Population Censuses and Surveys), but in the remaining 138 chemoprophylaxis was only apparent from the survey clinical form. In 280 cases the diagnosis was changed after notification, but only in around $50 \%$ of these is there evidence that the case was denotified. In 145 cases (mainly children) it was reported that two drugs only were to be given (for longer than three months) and it was uncertain if 
this represented therapy or prophylaxis. In 78 patients a standard chemotherapy regimen was given although the diagnosis was uncertain due to the absence of specific symptoms, signs or laboratory results. Finally, there is some uncertainty about whether refugees and short term visitors should be notified. Further recommendations on the reporting of tuberculosis will be discussed.

\section{Tuberculosis incidence in Scotland is increasing in the young and the elderly}

JS DUFFIELD, WH ADAMS, M ANDERSON, AG LEITCH Respiratory Medicine Unit, Royal Victoria Chest Clinic, RIE NHS Trust; Medical Statistics Unit, University of Edinburgh, Edinburgh Notifications of tuberculosis by age, sex and site of disease for the years 1981-92 were obtained from the Information and Statistics Division of the NHS in Scotland. Total, as well as age and sex related incidence rates, were calculated for each year using General Register Office population estimates. Total tuberculosis incidence rates per $10^{5}$ population for 1981-92 are shown in the table and demonstrate a decline succeeded by a plateau

\begin{tabular}{lllllllllllll}
\hline & \multicolumn{1}{l}{ Year } \\
\cline { 2 - 10 } & 81 & 82 & 83 & 84 & 85 & 86 & 87 & 88 & 89 & 90 & 91 & 92 \\
\hline Rate & 19.3 & 14.6 & 15.9 & 14.2 & 13.6 & 14.7 & 10.9 & 10.3 & 10.5 & 10.8 & 10.8 & 10.9 \\
\hline
\end{tabular}

in 1987. Age group analysis of pulmonary tuberculosis showed that incidence $\left(10^{-5}\right)$ decreased from 7.4 in 1981 to 2.6 in 1987 , rising significantly $(\mathrm{p}=0.012)$ by an estimated $12.6 \%$ per annum to 3.7 in 1992 in the $0-14$ age group. In the $65+$ age group incidence declined from 30.1 in 1981 to 17.3 in 1988 and rose significantly $(p=0.048)$ by an estimated $4 \cdot 1 \%$ per annum to $22 \cdot 2$ in 1992 . Age groups $15-44$ and 45-64 show a continuous decrease in rate on natural logarithmic scales from 11.2 and 23.8 in 1981 to 4.3 and 11.4 respectively in 1992. Inclusion of extrapulmonary cases to give total tuberculosis notification rates revealed almost identical findings within all age groups with the same patterns on regression analysis of incidence. The estimated annual increase in total incidence in 1992 was $8.5 \%$ $(p=0.035)$ for the $0-14$ age group and $2.8 \%(p=0.102)$ for the $65+$ age group. Pulmonary tuberculosis in Scotland is increasing significantly in the young and the elderly; the increases may be causally related.

\section{Characteristics and outcome of patients with pulmonary non- tuberculous mycobacterial infections}

L DAviES, PDo Davies Tuberculosis Research Unit, Aintree Chest Centre, Fazakerley Hospital, Liverpool Studies of patients with non-tuberculous mycobacterial infections have shown a high mortality. We performed a retrospective case study of all patients presenting to Aintree Hospitals, Liverpool over a six year period from January 1988 to December 1993 with culture proven non-tuberculous mycobacterial disease. There were 38 patients in total; seven were HIV positive and four had non-respiratory infections. These were excluded. Of the 27 remaining patients $18(67 \%)$ were men (age range 44-84, mean 66 years). A total of 19 subjects smoked at the time of diagnosis, seven were ex-smokers and one only was a lifelong non-smoker. Seven drank more than twice the recommended limit of units of alcohol per week. Fifteen patients had COPD with $\mathrm{FEV}_{1}$ values ranging from $24-84 \%$ of predicted (mean $55 \%$ ). Twelve $(44 \%)$ had evidence of other smoking related disease and nine (33\%) had a history of $M$ tuberculosis disease. At least two isolates were obtained from each patient, in 26 cases from sputum alone and, in the remaining patient, from bronchial washings. Fifteen (56\%) grew $M$ kansasii, six (22\%) $M A C$, five (18\%) $M$ malmoense, and one (4\%) $M$ xenopi. Of the 19 who smoked at the time of diagnosis, seven (37\%) died within the study period (four reportedly of COPD), three remained unwell with frequent admissions due to cough and breathlessness, six are now well, and three were lost to follow up. Of the seven who were exsmokers at the time of diagnosis four (57\%) died within the study period (three reportedly of COPD and one of $M$ kansasii), one remains breathless, one is now well, and one is lost to follow up. The one lifelong non-smoker responded rapidly to treatment and is now well.
Non-tuberculous mycobacterial infection appears to be predominantly a smoker's disease.

Tuberculosis screening and tuberculin testing in two social clubs in the city of Liverpool

PDO DAVIES, CSD WILliams, s JAMIESON, A STEEL, Q SYED, M BELLIS, N BEECHING Tuberculosis Research Unit, Regional Epidemiological Centre, $\overrightarrow{\overline{\vec{F}}}$ Infectious Diseases Unit, Aintree Hospitals, Liverpool In the course of $\vec{D}$ an investigation into an outbreak of tuberculosis (TB) in a social club in Liverpool in 1993198 club members were screened by chest $\bar{\sigma}$ radiography and Heaf testing. All those with a grade 3 and 4 were $\bar{\Phi}$ offered a chest radiograph. No further cases of TB were found. In $\overparen{\nabla}$ order to determine the background population of Heaf positivity within Liverpool a controlled study was done in a social club in a part of Liverpool where no cases of TB had been reported. $171 \overrightarrow{0}$ "controls" were tested. Percentage of Heaf grade for the two clubs is shown in the table. The control population was older than those from $\vec{\omega}$

\begin{tabular}{|c|c|c|c|c|c|}
\hline \multirow[b]{2}{*}{ Social club } & \multicolumn{5}{|c|}{ Heaf grade } \\
\hline & 0 & 1 & 2 & 3 & 4 \\
\hline $\begin{array}{l}\text { TB cases }(\%) \\
\text { No TB cases }(\%)\end{array}$ & $\begin{array}{l}12 \\
25\end{array}$ & $\begin{array}{l}11 \\
13\end{array}$ & $\begin{array}{l}36 \\
35\end{array}$ & $\begin{array}{l}20 \\
16\end{array}$ & $\begin{array}{l}21 \\
11\end{array}$ \\
\hline
\end{tabular}

the TB affected club. There was no statistical difference between those that had received BCG vaccination and those who had not. $\vec{\bullet}$ The club where TB cases had been reported showed a significantly of greater number of members who were Heaf grades 3 or $4(p<0.005)$. The study at the "control" club showed that a significant proportion (27\%) of adults are likely to have had previous TB infection. Adults attending the social club where there had been TB cases reported $\bar{\partial}$ showed a higher proportion (41\%) of members with Heaf grades 3 응 and 4 . It is probable that the difference in proportion of grade 3 and $\mathbb{D}$ 4 test positive subjects (14\%) represents recent infection.

\section{Tuberculin test screening in substance abusers}

PDO DAVIES, C MORRISON, D WAKEFIELD, SM RUBEN, N BEECHING O Tuberculosis Research Unit and Maryland Centre, Liverpool Drug users have been one of the most at risk groups from HIV infection due to $\bar{\sigma}$ their lifestyle through either the sharing of contaminated injecting equipment or sexual behaviour. Tuberculosis (TB) is often associated. Reports from the USA recommend that TB screening should be $\frac{0}{3}$ undertaken in substance abuse treatment centres as drug users will be at high risk because of the higher seroprevalence of HIV and 윽 adverse social factors. The Liverpool Drug Dependency Clinic is the $D$ largest clinic in Europe, treating over 1000 opiate addicts each year on a methadone programme. One hundred and eighteen drug users $\mathrm{N}$ had Heaf testing performed and read within 10 days. The mean (SD) of age was $28.4(5 \cdot 6)$ years (range $17-46)$. There were 77 males $(65 \% \mathrm{~N}$ of the 118). Ten $(8.4 \%)$ were homeless and $21(17 \cdot 8 \%)$ lived in households of five or more. Eighteen (15.3\%) reported having a family member who had had TB. There were four non-smokers and $<$ 70 non-drinkers, $17(14 \cdot 4 \%)$ drinking over 35 units a week. Seventeen

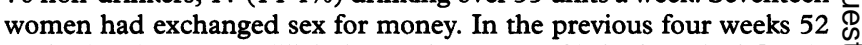
$(44 \%)$ had injected an illicit drug. Nineteen $(16 \%)$ had smoked Crack. Heaf test grade results showed that $26(22 \%)$ were grade $0,85(72 \%)$ grade 1 or 2 , and seven $(6.0 \%)$ grade 3 or 4 . Sex, age, homelessness, employment status, smoking, alcohol or drug use were not significantly associated with any Heaf grade result. Forty three $(36.4 \%)$ had never $\frac{\mathbb{Q}}{\mathscr{Q}}$ had school BCG vaccination and 20 of these had a grade 0 result. $\sigma$ Only previous BCG was associated with a positive Heaf test $(p<0.001)$. Only five of the 33 referred for BCG vaccination or further assessment at the TB clinic, attended. Risk of TB infection amongst Liverpool substance abusers is low.

Experience of miliary tuberculosis in a high incidence area: Blackburn 1978-93

LP ORMEROD Chest Clinic, Blackburn Royal Infirmary, Blackburn Lancs Thirty nine cases of miliary tuberculosis ( 25 cryptic: 14 
classical) were seen in a population of 265000 with over $10 \%$ of Indian subcontinent (ISC) ethnic origin over a 16 year period, giving an annual incidence of $0.92 / 100000$. Of the 14 classical cases three were white (mean age 51.3 years; range $2-79$ ), three Pakistani (mean age $35 \cdot 71 ; 23-54$ ), seven Indian (mean age 37 ; range $25-53$ ), and one Chinese (age 93). All had classical miliary $x$ ray shadowing, 5/ $14(36 \%)$ had associated TB meningitis with positive CSF cultures, five had positive sputum cultures and one positive histology from a skin lesion. Two died on treatment at four days (age 93, TBM) and six weeks (age 23), after commencement of therapy. Of the cryptic cases seven were white (mean age 52.7; range 19-76), 10 Pakistani (mean age $39 \cdot 5$; range $16-85$ ) and eight Indian (mean age 39.5; range $21-71)$. Twenty two of $25(88 \%)$ had a normal chest radiograph, one had old apical calcification, and two focal nodular pulmonary TB. Three patients (white aged 48, Pakistani aged 38, Indian aged 31) were all diagnosed at post-morten examination following deaths in the community. One other case was confirmed by positive sputum culture, the rest showed response to specific antituberculosis treatment and fulfilled Proudfoot's criteria (Proudfoot et al. BMF 1969;ii:273-6). The outcome with $5 / 39$ (13\%) mortality and $25 / 39$ (64\%) cryptic miliary TB is substantially different from the spectrum and outcome of disease reported from Edinburgh (Sime et al. Am Rev Respir Dis 1993;147:A121) which has a minimal immigrant community. The better outcome in a high prevalence, high immigrant area probably reflects greater awareness of the diagnosis and prompter initiation of treatment.

Effects of winter pollution on respiratory symptoms and medication use in subjects with asthma and chronic bronchitis

SCO TAGGART, HC FRANCIS, AM FLETCHER, A CUSTOVIC, BG HIGGINS, A wooDcock Wythenshawe Hospital, Manchester We have previously reported on the effects of wintertime air pollution on measurements of peak flow in subjects with asthma and chronic bronchitis (Thorax $1994 ; 49: 360 \mathrm{P})$. Here we report on the results of the analysis of symptoms and medication use in the same group of subjects. For one month during January-February 1992 subjects recorded their daily bronchodilator use (BU) and symptoms of wheeze, dyspnoea, cough, throat and eye irritation were scored on a visual analogue scale. Daily means of $\mathrm{SO}_{2}, \mathrm{NO}_{2}$ and $\mathrm{O}_{3}$ were measured by absorption spectroscopy. After allowing for between subject differences and controlling for temperature, multiple logistic regression was used to investigate whether subjects complained of wheeze, etc and used more than their average BU as levels of same day, 24 and 48 hour lags of pollution increased. Satisfactory records were received from 50 subjects (21 reactors with methacholine $\mathrm{PD}_{20} \mathrm{FEV}_{1}<12.25 \mu$ mol identified). Max daily mean $\mathrm{SO}_{2}$ and $\mathrm{NO}_{2}\left(169,151 \mu \mathrm{g} / \mathrm{m}^{3}\right)$ exceeded guidelines for health, $\mathrm{O}_{3} 41 \mu \mathrm{g} / \mathrm{m}^{3}$. Same day $\mathrm{O}_{3}$ was associated with increased BU in all subjects $(\mathrm{p}<0.01)$ and in reactors a trend was observed between 48 hour lag $\mathrm{O}_{3}$ and dyspnoea $(\mathrm{p}<0.06)$. No other adverse effects were found. We conclude that, at the levels encountered, the winter pollutants measured have little effect on respiratory symptoms in this group of potentially more sensitive individuals. As in a previous summer study (Am Rev Respir Dis 1993;147:A637) BU is again associated with ambient $\mathrm{O}_{3}$ levels.

\section{Toxicity of environmental particulate: studies with ultrafine} titanium dioxide

K DONALDSON, P GILMOUR, W MACNEE Biomedicine and Biotechnology Research Group, Napier University, Edinburgh; Unit of Respiratory Medicine, Department of Medicine, University of Edinburgh Epidemiological studies have demonstrated a firm relationship between the levels of fine particles suspended in environmental air $\left(\mathrm{PM}_{10}\right)$ and (a) respiratory and cardiovascular mortality; (b) attacks of asthma and COPD. $\mathrm{PM}_{10}$ particulate cannot be obtained in sufficient quantities for experimentation and so we utilised two samples of the "inert" pigment titanium dioxide: normal $\mathrm{TiO}_{2}\left(\mathrm{NTiO}_{2}\right) 250 \mathrm{~nm}$ diameter and ultrafine $\mathrm{TiO}_{2}\left(\mathrm{UFTiO}_{2}\right) 25 \mathrm{~nm}$ diameter (Ferin et al. Am $\mathcal{F}$ Respir Cell Mol Biol 1992;6:535-42), the latter being a surrogate for the fine environmental particles. We added $\mathrm{NTiO}_{2}$ and $\mathrm{UFTiO}_{2}$ to cells of an epithelial cell line (A549) for four hours and found that $\mathrm{UFTiO}_{2}$ caused dose-dependent detachment of the cells from their substratum, without toxicity, whilst $\mathrm{NTiO}_{2}$ had very little effect: data as mean (SE) $\mathrm{cpm}$ in detached cells in triplicate wells minus control value in four separate experiments: $50 \mu \mathrm{g} / \mathrm{ml} \mathrm{NTiO} 282(144) ; 50 \mu \mathrm{g} / \mathrm{ml} \mathrm{UF}$ $\mathrm{TiO}_{2} 1018$ (667); $\mathrm{p}<0 \cdot 05$. The ability to nick supercoiled DNA, detected by quantitative scanning laser densitometry of ethidium bromide-stained agarose electrophoresis gels, is a sensitive assay for the ability of particle surfaces to release free radicals. $\mathrm{UFTiO}_{2}$ caused $100 \%$ supercoiled DNA strand breakage (three separate experiments) whilst $\mathrm{NTiO}_{2}$ had no effect. The effect of $\mathrm{UFTiO}_{2}$ was greater than we have seen with quartz or asbestos and was partly mediated by hydroxyl radicals. A substantial fraction of environmental particulate is in the ultrafine ( $<50 \mathrm{~nm}$ ) size range and, if these have the same activities as shown here for the $\mathrm{UFTiO}_{2}$, then this could help explain why $\mathrm{PM}_{10}$ is so toxic.

\section{Occupational asthma due to a widely used soft solder flux not} containing colophony

RP CONVERY, RJ WARD, DJ HENDRICK Chest Unit and Regional Unit for Occupational Lung Disease, Newcastle General Hospital, University of Newcastle upon Tyne Telux flux is a safe alternative to colophony soldering flux, containing zinc and ammonium chlorides as its main active constituents. We describe flux fume induced asthma in a previously well 19 year old female gas appliance servicing engineer. The initial presentation was the development of a viral-like illness after 10 weeks regular work exposure to Telux flux. She then noted several episodes of wheeze and exertional dyspnoea which appeared work related. Her subsequent work environment was changed so as to minimise exposure before further investigation. Atopy was diagnosed (skin reaction to house dust mite) and sibling histories of childhood asthma and hayfever were noted. An initial methacholine test (Newcastle protocol) showed a high degree of airway responsiveness (AR) with a $\mathrm{PD}_{20}$ of $10 \mu \mathrm{g}$, despite her remaining relatively asymptomatic Four months after exposure ceased $\mathrm{PD}_{20}$ had markedly increased to $1159 \mu \mathrm{g}$. Laboratory based inhalation provocation tests were performed with 30 and then 60 minutes of flux fume exposure while soldering. The 30 minute exposure test had the baseline $\mathrm{FEV}_{1}$ falling by $25 \%$ but only just meeting the $95 \%$ lower confidence interval The $\mathrm{FEV}_{1}$ area decrement (AD) was increased significantly from 0.06 to 4.92 litre hours $(\mathrm{p}<0.01)$ and $\mathrm{PD}_{20}$ fell from 800 to $100 \mu \mathrm{g}$. The 60 minute test saw the baseline $\mathrm{FEV}_{1}$ fall by $58 \%$ during the late asthmatic reaction (LAR) but again there were minimal symptoms. The area decrement was increased significantly from 0.033 to 21.5 litre hours $(\mathrm{p}<0.001)$ and again $\mathrm{PD}_{20}$ fell dramatically from 1706 to $34 \mu \mathrm{g}$. Two weeks after the last challenge test $\mathbf{P D}_{20}$ had risen to $2094 \mu \mathrm{g}$, a value which is not usually associated with active asthma. Zinc chloride has previously been implicated in soft solder asthma (Thorax $1989 ; 44: 220-3$ ) and it is likely that any mineral oils presen will burn off in the soldering process. The immediate and late falls in $\mathrm{FEV}_{1}$ and increases in AR demonstrate, despite minimal symptoms, that Telux is capable of inducing occupational asthma. This case also demonstrates the wide variation in AR typical of early stages of the illness. We suspect the zinc chloride component of the flux acting as a sensitisation agent in certain individuals.

Influence of a variety of personal characteristics upon across shift and across week changes in lung function in textile workers

CJ WARBURTON, AM FLETCHER, CAC PICKERING, RM NIVEN, LA OLDHAM, HC FRANCIS Northwest Lung Centre, Wythenshawe Hospital, Manchester One hundred and ninety six textile workers performed spirometry and bronchial reactivity (BR) before and after their first and fourth working shifts of the week. The effect of several factors upon across shift and across week changes (day 1 pre-shift minus day 4 pre-shift) in spirometry and BR was studied. No differences were found between men and women, whites and Asian subpopulations. Cotton workers (as opposed to manmade fibre workers (MMF)) had significantly lower percentage of predicted spirometry and significantly higher baseline BR $(p<0.05)$. No differences between the two groups for across shift and across week changes could be demonstrated. A high cotton exposure group demonstrated a significant increase in BR across shift on day 1 as compared with a low exposure group $(\mathrm{p}<0.05)$, but there were no other differences. Operatives who had ever smoked (ES) showed a significantly lower \% predicted spirometry and higher baseline BR than the lifelong non-smoker (NS) group $(p<0 \cdot 05)$. The ES group demonstrated significantly greater across week falls in all spirometric parameters compared to the NS group. None of the personal characteristics seemed important in the production of across shift changes in lung function; however, cigarette smoking was the most important one involved in the production of changes in spirometry across the working week in these textile workers. 
Acclerated lung function decline in textile workers: cotton dust, cigarette smoking or both?

CJ WARBURTON, AM FLETCHER, CAC PICKERING, RM NIVEN, LA OLDHAM, HC FRANCIS Northwest Lung Centre, Wythenshawe Hospital, Manchester One hundred and ninety six Lancashire textile workers performed spirometry before the first shift of the working week as part of an ongoing study of across week variations in lung function. Percentage of predicted lung function was calculated using the regression equations of Quanjer (Bull Eur Physiopathol Respir, 1983) for whites, and our own previously presented regression equations for Asians (Warburton et al. Thorax 1994). Correlations between various predictor parameters and percentage predicted lung function were calculated (SPSS) with 194 degrees of freedom. Significant negative correlations between $\mathrm{PPFEV}_{1}$ and $\mathrm{PPFEF}_{25-75}$ and (1) the cumulative number of cigarettes smoked (cigyrs), (2) the number of years spent working with cotton dust, and (3) the number of work-related respiratory symptoms present in each operative were found. PPFEV, PPFVC and PPFEF P5-75 $_{25}$ were all significantly negatively correlated with age (the influence of which should have been removed by the regression equations). These results suggest that accelerated decline in the spirometric indicators of bronchial obstruction $\left(\mathrm{FEV}_{1}, \mathrm{FEF}_{25-75}\right)$ are related to cigarette smoking and to the number of years of exposure to cotton dust. These two factors may be interrelated and the relative importance of each is difficult to ascertain. The strong correlations with age may suggest that, as operatives age, the effects of longer cotton dust exposure and cumulatively more cigarettes are at least additive.

\section{Airborne house dust mite allergen}

A custovic, sco taggart, a woodcock North West Lung Centre, Wythenshawe Hospital, Manchester Airborne sampling is widely used in the assessment of occupational exposure and might be considered as a more representative measure of inhaled allergen than reservoir levels. However, its use as a method for house dust mite allergen measurement is still controversial. Personal airborne samples were collected with Casella sampler $(2 \mathrm{l} / \mathrm{min})$ : overnight in bed with a modified face mask ( 8 hours; $n=7$ ); during daily activities ( 8 hours; $\mathrm{n}=7)$; and during laboratory sieving of domestic dust samples $(2.5$ hours; $n=8)$ ). Fixed location samples were collected with a high volume sampler $(60 \mathrm{l} / \mathrm{min})$ before and after disturbance ( 4 hours; $n=$ 8 ); overnight with Casella sampler attached to pillow ( 8 hours; $n=$ 30) [mattress and bedding Der $p$ I: geometric mean (GM) 9320 $\mathrm{ng} / \mathrm{g}$ ]. $\operatorname{Der} p$ I was undetectable $(<1 \mathrm{ng} / \mathrm{ml})$ in all personal airborne samples collected overnight and during the usual domestic activities (mean sample volumes (MSV) $0.93 \mathrm{~m}^{3}$ and $0.96 \mathrm{~m}^{3}$, respectively) High levels of airborne Der $p$ I were measured during sieving of dust (GM $38.9 \mathrm{ng} / \mathrm{m}^{3}$, range 18.3-72.6; MSV 0.3 $\mathrm{m}^{3}$ ). Der $p$ I was undetectable in all fixed location samples collected before disturbance (MSV $15 \cdot 5 \mathrm{~m}^{3}$ ). Detectable levels $(>1 \mathrm{ng} / \mathrm{ml}$ but $<4 \mathrm{ng} / \mathrm{ml}$; MSV $15.5 \mathrm{~m}^{3}$ ) were identified in all samples after disturbance. Der $p$ I was barely detectable ( $>1 \mathrm{ng} / \mathrm{ml}$ but $<2 \mathrm{ng} / \mathrm{ml}$ ) in $3 / 30$ and undetectable in $27 / 30$ overnight samples (MSV $0.95 \mathrm{~m}^{3}$ ). As yet, airborne sampling is insufficiently sensitive to produce reliable and repeatable results. More sensitive assays or better sampling methods are needed.

\section{Levels of exposure to mite allergen in hospital wards}

AM FLETCHER, A CUSTOVIC, CAC PICKERING, H FRANCIS, AA woodcock North West Lung Centre, Wythenshawe Hospital, Manchester House dust mites are generally accepted as a major source of indoor allergen. In 1994 Mohindra et al. (Clin Exp Allergy 1994;24:174) reported levels of house dust mite allergen recovered from carpeted wards in excess of those considered to be a risk factor for acute asthmatic attacks. In this study three hospitals were investigated. The investigation included four carpeted wards in two NHS hospitals and the carpeted single rooms of a local private hospital. The age of the carpets in the wards varied between 18 months and 10 years. Fine dust samples were collected by vacuuming (flow $45 \mathrm{l} / \mathrm{s}$ ) $1 \mathrm{~m}^{2}$ of carpet and mattress for two minutes. The $\operatorname{Der} p$ I antigen content was assayed by a two-site immunometric ELISA In total 21 mattresses and 26 carpets dust samples were analysed. The mite allergen levels were found to be low in all three hospitals in all sample areas. The geometric mean level of Der $p$ I antigen collected from the mattresses was $234 \mathrm{ng} / \mathrm{g}$ (range $100-1200 \mathrm{ng} / \mathrm{g}$ ) and from the carpets $138 \mathrm{ng} / \mathrm{g}$ (range $100-410 \mathrm{ng} / \mathrm{g}$ ). In one hospital dust was also collected from the surface of six fabric covered chairs stituated in the outpatient area. These samples provided the highest levels of mite allergen; geometric mean $975 \mathrm{ng} / \mathrm{g}$ (range 680-1200 $\mathrm{ng} / \mathrm{g}$ ). This study demonstrates that mite allergen levels in carpeted hospital wards and on sealed hospital mattresses are at a low level and are unlikely to be of clinical significance to house dust mite sensitive asthmatic patients.

Levels of contamination of domestic dust with endotoxin and $\overrightarrow{\overline{\vec{S}}}$ Der $p$ I

JCG SIMPSON, A CUSTOVIC, CAC PICKERING, AM FLETCHER, R MCL NIVEN, $\frac{\bar{\sigma}}{\bar{c}}$ LA OldHAM, AA WoODCock North West Lung Centre, Wythenshawe $\overparen{\mathbb{D}}$ Hospital, Manchester Environmental factors are clearly important in the pathogenesis of asthma. While domestic allergens are of primary importance, a wide variety of indoor and outdoor pollutants may have $\vec{\circ}$ a role in modifying the asthmatic response. Endotoxins are derived :from the cell wall of Gram negative bacteria and are potent pro- $\vec{\omega}$ inflammatory agents. They are found to be contaminants of various occupational environments and are implicated as aetiological agents $\overrightarrow{\vec{\partial}}$ in a number of diseases (including byssinosis, organic dust toxic syndrome, and humidifier fever). We investigated the contamination of domestic dusts with endotoxin and mite allergen $\operatorname{Der} p \mathrm{I}$ in 13 houses. Paired fine dust samples were collected by vacuuming (flow $\vec{\omega}$ $45 \mathrm{l} / \mathrm{s}) 1 \mathrm{~m}^{2}$ of mattress and living room carpet for two minutes. $\vec{\nabla}$ Samples were assayed for endotoxin (turbidimetric method using the 0 LAL 5000e machine) and Der p I (two site immunometric ELISA). The mean (SD) level of endotoxin collected from the carpets (expressed as $\mathrm{ng} / \mathrm{mg}$ of dust) was $14.8(23.9)$ and was significantly greater $\frac{7}{0}$ than from the mattresses $2 \cdot 8(6 \cdot 1) ; \mathrm{p}=0.001$. Der $p$ I levels (expressed as $\mathrm{ng} / \mathrm{g}$ of dust) were: carpets $7395(17651)$ and mattresses $5493 \vec{\bullet}$ (10 195). No correlation between measured endotoxin and $\operatorname{Der} p$ ㅇ I levels was found. This study documents the contamination of the domestic environment with endotoxin. Further work is indicated to ascertain the role of this potential exposure in asthma and specifically to determine any synergistic activity with allergen exposure.

Allergic bronchopulmonary aspergillosis after lung trans- $\overline{\bar{O}}$ plantation?

JJ EGAN, N YONAN, KB CARROLL, AK DEIRANIYA, AK WEBB, AA woodcock North West Lung Centre, Wythenshawe Hospital, Manchester Experience in lung transplantation suggests that asthma 0 is an organ specific condition. We describe two cases of allergic bronchopulmonary aspergillosis following lung transplantation for $\bar{\sigma}$ cystic fibrosis (CF). Patient A: a 37 year old male CF patient received a heart/lung transplant. The 18 year old donor had no history of $\delta$ asthma. Preoperatively the recipient was negative for Aspergillus precipitins (Asp pptns) and he was Aspergillus fumigatus ( $A s p f$ ) sputum negative. One year after surgery he was weaned off steroids. Three years postoperatively he presented with a wheeze, a peripheral eos- $D$ inophilia of $4 \times 10^{9}$ (normal<0.4 $\times 10^{9}$ ), skin tests were positive to $A s p$ 을 $f$ only, total $\mathrm{IgE}=70 \mathrm{IU} / 1$ (normal $<200$ ), RAST to $A s p f$ was positive $N$ at four units (normal <0.35). HRCT showed areas of collapse and $G$ consolidation. At bronchoscopy mucus plugs were aspirated and $A s p$ N $f$ was cultured. He responded to prednisolone and inhaled fluticasone. N Patient B: A 20 year old male patient received a double sequential $\sigma$ lung transplant for CF. The recipient was atopic with positive skin tests to $\operatorname{Der} p \mathrm{I}$, grass and $A s p f$. Prior to transplant $A s p f$ was not $\frac{0}{C}$ isolated from his sputum and he was $A s p$ pptns negative. The 15 year old donor had mild asthma. Four months after surgery he presented with complete collapse of the left lung. Bronchoscopy revealed mucus plugging of the left bronchial tree. The plugs contained eosinophils and fungal hyphae. He had a peripheral eosinophilia of $1.1 \times 10^{9}, \stackrel{\mathbb{D}}{\mathrm{Q}}$ total $\mathrm{IgE}=1500 \mathrm{IU} / \mathrm{l}(<200)$ and RAST to $A s p f$ was 55 units $(<0 \cdot 35)$. Methylprednisolone $500 \mathrm{mg}$ for three days and nebulised budesonide daily resulted in a rapid resolution of the radiological and bronchoscopic findings. Effective control required prednisolone $60 \mathrm{mg} /$ o day covered by oral itraconazole and lyposomal amphotericin. These cases indicate that both systemic and local mechanisms can contribute to an allergic reaction in the airways.

Audit of an admission data sheet for patients with acute asthma

P HUGHES, C SHEPHERD, CR MCGAvIN The Chest Clinic, Freedom Fields Hospital, Plymouth Assessment of acute asthma relies on measure- 
ment of a number of clinical variables. However, retrospective studies of asthma admissions show that only pulse and peak flow are reliably recorded (Bell et al. BMF 1991;302:1440-3; Littlejohn et al. Postgrad Med $\mathcal{f}$ 1994;70:92-9). We have designed an admission proforma for patients with asthma admitted via the general medical admissions ward. The structure of the proforma encourages recording of a concise history, with clinical findings known to be of value. Advice on immediate management, based on the BTS guidelines, is also included. Between February and July 1993109 admissions coded as acute asthma were identified. A trained audit clerk assessed how frequently selected details of history, examination and investigations had been recorded. The admission data sheet had been used in 23 cases $(21 \%)$, the routine medical clerking notes being used in the remainder. By using a modified $t$ test we found significant differences $(p<0.05)$ in recording of peak flow when well, number of previous admissions, time since last attack, and time of admission. The recording of cyanosis, pulse, blood pressure, peak flow and paradox were also significantly improved $(p<0.05)$, as were results of blood gas analysis with details of inspired oxygen concentration. The data sheet leads to a focused efficient assessment of an acute asthma attack, and has been found to facilitate management of the asthmatic patient.

\section{Audit of emergency self-admission for asthma}

VA VORA, JE SCULLION, MDL MORGAN, AJ WARDLAW Department of Respiratory Medicine, Glenfield Hospital, Leicester The BTS guidelines for asthma stress the need for an emergency self-admission service for patients at risk. A dedicated emergency bed for this purpose has been provided in Leicester since 1984. To assess the quality and appropriateness of this service we have audited admissions for a five month period between April and August 1993. The admitting nursing and medical staff collected the following data for each admission: interval between notification, arrival and initiation of therapy; mode of transport; reason for admission (related/unrelated to asthma); and assessment of severity by estimation of peak flow (PEF) and arterial blood gases (ABG). Of the 147 patients on the self-admission list, 47 were admitted during the 153 days of the study period. Thus, 106 bed days $(70 \%)$ remained unoccupied. More than $50 \%(26 / 47)$ transported themselves to hospital. Mean interval between notification and arrival was 52 minutes ( $R=5 \mathrm{~min}$ to 2.5 hours), and between arrival and initiation of treatment was 20 minutes $(R=0-2 \cdot 3$ hours). Seven patients were admitted with problems unrelated to acute asthma. Arterial blood gas tensions were normal in $17(36 \%)$ patients and PEF was within expected range in two patients. There were no admissions to ITU or fatalities amongst patients on the self-admission list during the study period. In conclusion, even though self management plans were not universally applied, $74 \%$ of admissions were deemed appropriate by the admitting staff, but the bed occupancy was only $30 \%$ (normal bed occupancy $100 \%$ ). Therefore the selfadmission system was felt to fulfil a need but the cost should be recognised in the contracting process.

\section{New asthma referrals to hospital clinics}

I RYLAND, MG PEARSON ON BEHALF OF THE NATIONAL ASTHMA TASK FORCE AND 30 participating physicians Aintree Chest Centre, Liverpool There are few data about the patterns of referral to, or what happens in, hospital outpatient clinics. The National Asthma Task Force has surveyed 21 adult and nine paediatric hospitals collecting data on 333 new adult patients and 152 new paediatric referrals. Median age of adults was $43(13-85)$ years and children 3 (3 months -14 ) years. $57 \%$ of adults and $39 \%$ of children were female; $259 / 296(88 \%)$ adults and $121 / 144(84 \%)$ children were directly referred by the GP. For adults, of the 259 GP letters, $73(28 \%)$ requested help with diagnosis, $132(51 \%)$ asked for advice on treatment, and $22(8 \%)$ indicated patient pressure for referral. In children corresponding figures are 55/122 (45\%), 55/122 (45\%), and 7/122 (6\%), respectively. Treatment on referral together with the treatment recommended after consultation is shown in the table. For adults the

\begin{tabular}{llllll}
\hline & \multicolumn{2}{l}{$\%$ Adults } & & \multicolumn{2}{l}{$\%$ Children } \\
\cline { 2 - 3 } \cline { 5 - 6 } \cline { 5 - 6 } & Before & After & & Before & After \\
\hline No anti-inflammatory & 21 & 8 & & 51 & 12 \\
SCG/nedocromil & & & 22 & 30 \\
Low dose inhaled steroid & 46 & 30 & & 23 & 20 \\
High dose inhaled steroid & 27 & 56 & & 4 & 32 \\
Oral steroids & 5 & 5 & & 0 & 5 \\
\hline
\end{tabular}

first outpatient consultation increased treatment in 106/299 (46\%), left it unchanged in $104(45 \%)$, and reduced it in $19(8 \%)$. For children treatment was stepped up in $40 \%$, unchanged in $48 \%$, and down in $12 \%$. Although $52 \%$ of adults and $40 \%$ of children reported waking with asthma for two or more nights in the last week, the changes in treatment were not related to these symptoms. Adult physicians discharged $17 \%$ at first visit and intended to discharge another $53 \%$ within six months. Of those intended to be followed for more than a year, $51 / 64(80 \%)$ were on high dose inhaled steroids at least. Paediatricians discharged $21 \%$ at visit 1 and planned early discharge in another $31 \%$. Most asthma patients are referred by GPs, half have their treatment increased at first visit and early discharge back to GP is planned in $70 \%$ of adults and $52 \%$ of children. Most physicians intend long term follow up only for the most severe asthma patients. [Supported by a grant from the Department of Health.]

\section{Asthma and poverty in the West Midlands}

IP WATSON, P COWAN, RA LEWIS Worcester Royal Infirmary, Worcester The suspected link between asthma and poverty was examined in a retrospective study of hospital admission rate for asthma (corrected for population) and Townsend deprivation indices based on the 1991 census in the 18 districts in the West Midlands Regional Health Authority. A total of 27117 finished consultant episodes for asthma were studied. There was a strong correlation between the hospital admission rate for asthma and the Townsend Index for each district $(r=0.82, \mathrm{p}<0.001)$ (figure). The association was consistent

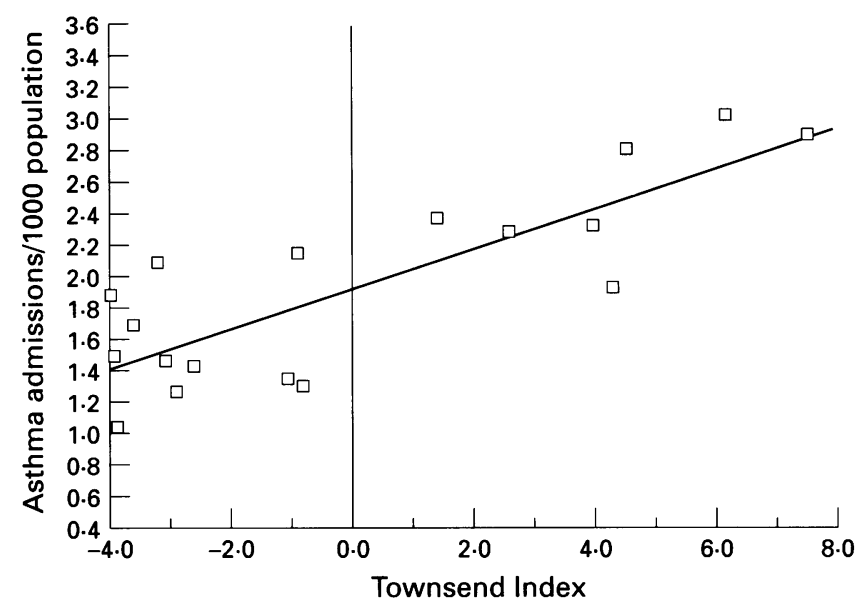

for all age groups and remained equally strong when predominantly rural districts were excluded from the analysis. Poverty is associated with an increased incidence of hospital admissions for asthma. This is not simply due to the urban environment, suggesting that factors other than exposure to pollution are important.

Quality of life assessment in asthma patients: development and validation of a questionnaire

DT BROWN, P GRANT, J PORTLOCK, E NEVILLE School of Pharmacy and Biomedical Sciences, University of Portsmouth, Portsmouth; Chest Clinic, St Mary's Hospital, Portsmouth Health related quality of life (QOL) is a concept which is gaining increasing importance in the management of chronic diseases such as asthma. A 20 item questionnaire was designed with Likert scale responses to five different life dimensions: physical activities, role activities, emotions, social activities and health perceptions. Positive and negative items were balanced within each dimension. It was administered by interview to 70 asthma patients ( 35 hospital clinic and 35 community clinic) with varying severity of disease as judged by clinical criteria and 69 healthy individuals from the general population, matched for age, gender and socioeconomic status. Internal consistency for the questionnaire was demonstrated by a high value of Cronbach's alpha (alpha $=0.94, \mathrm{n}=139$ ) and by statistically significant dimension-total correlations. Product moment correlations between the physical activities dimension score and physical measures of asthma severity (peak flow, forced vital capacity, and forced expiratory volume in one second) were statistically, highly significant. The control group established a calibration profile of typical QOL scores for the general population. Mean QOL scores for 
the control group was $84.37 \%$ compared with $81.99 \%$ for mild, $67.22 \%$ for moderate and $40.80 \%$ for severe asthmatics. The questionnaire is capable of rapid administration by a physician or asthma care nurse, provides an overall QOL score which is easy to calculate and interpret for individual patients, and discriminates between patients with differing disease severity.

\section{School absence and asthma: time for reappraisal}

C MCCOWAN, RG NEVILLE, FP BRYCE, R CLARK, IK CROMBIE Departments of General Practice, Respiratory Medicine and Epidemiology, University of Dundee, Dundee Asthma was regarded as a major cause of school absence in the 1970s and 1980s. A follow up, for up to three school terms, of a cohort of children drawn from 12 rural, town and urban general practices in Tayside gave the opportunity to reappraise absence and asthma. School absence in a group of 351 primary and secondary school children, taking anti-asthma treatment (British Thoracic Society steps) were compared with a group of non-asthmatic children, matched for age, sex and school class, to measure the effect of asthma on school absence. Working through the Education Department class registers for individual schools were examined and the number of halfdays absent and episodes of absence were recorded for children in the two groups. Subgroups of boys and girls, and primary and secondary school subjects were compared. The study group was also split into subgroups representing each step of asthma treatment and comparisons made between these groups. There was no significant increase in school absence, either halfdays missed or episodes of absence, for the children on anti-asthma treatment when compared with the matched controls. There was no difference in the amount of school absence dependent on severity of asthma as defined by the British Thoracic Society steps of treatment. In the early 1990s treated asthma, classified by the British Thoracic Society treatment steps, would not seem to be a major cause of school absence in its own right. Children with asthma who miss school are likely to have another reason for their absence.

\section{Care of children with asthma in schools}

K mansfield, hW Clague, ej PUgh, pJB mattinson Asthma Task Force, Co. Durham Health Commission, Appleton House, Durham The potential for the health and education services to form an alliance to address asthma management in children has been explored by a questionnaire directed to head teachers of all 296 primary and 43 secondary state schools in Co. Durham during January 1994. Head teachers were asked about a school policy for asthma, the availability of inhaler medication, the adequacy of training in the management of asthma, and whether further training would be welcomed. Responses were received from $22(76 \%)$ of primary and $28(65 \%)$ of secondary schools. Just $40 \%$ of schools had a policy regarding care of children with asthma. Within primary schools $60 \%$ of schools allow children to be responsible for their own inhaler compared with all but one secondary school. Inhalers were locked away in $27 \%$ of primary schools. A third of schools had a member of staff trained to deal with asthma and $55 \%$ of primary and $71 \%$ of secondary schools had a member of staff who the head teacher felt could deal with an acute asthma attack. Less than half of primary and a third of secondary school teachers were aware of the range of inhalers available. In only 105 of 252 schools did the head teacher feel that the school nurse had input to the care of children with asthma. The majority of schools were interested in further training. The survey reinforces the findings in earlier studies which identify a need to develop school policies for asthma care and training of teachers. This could be achieved by closer collaboration between the health and education services.

Impact of structured nurse-led discharge planning on outcome in children hospitalised with acute asthma: a randomised controlled study

P MADGE, JY PATON Department of Child Health, University of Glasgow, Yorkhill NHS Trust, Glasgow In a one year prospective audit of acute asthma care in children we identified a high incidence of poor outcomes including ongoing asthmatic symptoms and a readmission rate of $20 \%$. This occurred despite over $93 \%$ of children receiving appropriate therapy with inhaled bronchodilators and oral corticosteroids. However, less than $10 \%$ of children had adequate discharge planning. In the present randomised, controlled study we evaluated the impact of a brief structured nurse-led discharge planning on subsequent asthma outcome. Children over two years during their first admission in the study period were randomly assigned to either a control or an intervention group. The intervention group received a specially designed information booklet on asthma, a review discussion session, a written management plan including a three day course of $\overline{\widehat{\gamma}}$ oral prednisolone, a two week follow up at a nurse-run asthma clinic, and access to telephone support. The control group received current $x$ standard medical care. Outcome was assessed by a postal questionnaire at 4-6 weeks after discharge, by reported reattendance for $\stackrel{\omega}{\sigma}$ nebulised bronchodilator, or by readmission to hospital over the $\bar{C}$ subsequent period (to date 8 months). There was improvement in $\bar{O}$

\begin{tabular}{lll}
\hline Outcomes & $\begin{array}{l}\text { Controls } \\
(n=40)\end{array}$ & $\begin{array}{l}\text { Intervention } \\
(n=35)\end{array}$ \\
\hline $\begin{array}{l}\text { Reattendance for nebulised bronchodilator } \\
\text { Readmissions for acute asthma }\end{array}$ & $\begin{array}{c}9(22 \cdot 5 \%) \\
10(25 \%)\end{array}$ & $\begin{array}{l}5(14 \cdot 3 \%) \\
1(2 \cdot 9 \%)\end{array}$ \\
\hline
\end{tabular}

night cough $(p=0.045)$, day time wheeze $(p=0.07)$, and breathless on exertion $(p=0.028)$ in the intervention group. Reattendances and readmissions had also decreased. These preliminary results provide $\vec{\omega}$ clear evidence that structured nurse-led discharge planning can have $\vec{\nabla}$ a substantial impact on ongoing asthma morbidity in childhood.

Changes in lung function in asthmatic children undergoing
general anaesthesia

HC ROMER, HA MAY, DP HEAF, RL SMYTH Department of Anaesthesia and Respiratory Unit, Royal Liverpool Children's Hospital, Alder Hey, § Liverpool Little is known of the effects of general anaesthesia on the lung function of asthmatic children. We studied 20 children with asthma and 20 children in whom asthma had been excluded, who were undergoing routine elective surgery. The children, aged between $\triangle$ five and 15 years, were matched for age, sex and type of operation. $\overrightarrow{\overrightarrow{0}}$ Spirometry was performed preoperatively and twice postoperatively 3 - "early" (the day of surgery) and "late" (the following morning.) Continuous overnight pulse oximetry was also recorded. We found no difference in preoperative peak expiratory flow rate (PEFR) or forced expiratory volume in one second $\left(\mathrm{FEV}_{1}\right)$ between the two $\vec{\partial}$ groups. Both groups showed significant deteriorations in $\mathrm{FEV}_{1}$ and PEFR in the early postoperative period. Asthmatics showed significant $x$ deterioration in $\mathrm{FEV}_{1}$ in the late postoperative period, whereas the control group did not. There were no significant differences in deterioration in lung function following anaesthesia between the two groups. Overnight pulse oximetry in asthmatics showed a greater range - reaching lower saturations - than in controls and time spent $ᄋ$ at saturations lower than $90 \%$ was markedly greater in asthmatics. We have demonstrated that well controlled children with asthma, undergoing anaesthesia for elective surgery, demonstrate a deterioration in lung function and overnight oxygen saturation. While $\mathrm{N}$ this did not cause any clinical problems in the group we studied, $N$ under less well controlled conditions adverse consequences may arise.

Five year follow up of patients involved in the $1989 Q$ fever outbreak

N FLINT, G SMITH, JG AYRES Environmental Heath, University of Bir- 무 mingham; PHLS, Birmingham Heartlands Hospital; Chest Research Institute, Birmingham Heartlands Hospital, Birmingham In 1989 one of $\mathbb{\mathbb { Q }}$ the largest ever outbreaks of $Q$ fever occurred in an area to the south of the Birmingham area. In all, 147 cases were recognised, the outbreak affecting predominantly men of working age. No deaths 8 were recorded in the acute phase, but two developed carditis and two renal failure requiring dialysis. Five years after the outbreak a follow up study was performed by postal questionnaire. A total of 114 cases for whom an address was known were circulated asking each to nominate a "buddy control" for a case control study. Replies were received from $93(82 \%)$ patients, 37 of whom nominated a control. Fourteen had left the address, five had died, leaving 74 (61 men $(84 \%)$ ) for analysis. Mean age was 53 years (range 13-83), the sample being representative of the original group. Chronic symptoms were common with $69 \%$ complaining of joint pains, $66 \%$ chronic fatigue, 
and $65 \%$ sleep disturbance. Cough (59\%), irritability (54\%), sweating $(53 \%)$, memory disturbance $(53 \%)$, and chest pain $(51 \%)$ were complained of by more than half. Cough was complained of by $26 /$ $48(54 \%)$ non-smokers and breathlessness by $24 / 48$ (50\%) nonsmokers, neither significantly different from the symptom pattern in smokers. The case control results are being analysed. Of the five deaths two were due to cardiomyopathy possibly due to $Q$ fever. These findings support findings from Australia that chronic $Q$ fever fatigue syndrome is a real entity. Further investigation of this unique cohort is essential to investigate ongoing morbidity and possible mechanisms of this syndrome.

Case control study of clinical features associated with ampicillin resistant Haemophilus infuenzae infection

S JOHNSON, RCF THOMPSON, JT MACFARLANE, H HUMPHREYS Respiratory Medicine, City Hospital, Nottingham; PHL, University Hospital, Nottingham Resistance to ampicillin amongst Haemophilus influenzae (HI) isolates in the UK has been steadily rising over the past decade, reaching over $8 \%$ in 1991 . The consequences of this trend in clinical practice have yet to be studied. We performed a case control study of respiratory infection with $\mathrm{HI}$ comparing 23 patients with an ampicillin resistant isolate (ARI) and 34 controls with an ampicillin sensitive isolate (ASI). Isolates were identified from representative sputum samples cultured using standard techniques over a three year period. Chronic obstructive pulmonary disease was the most common chest disease in both groups, $15 / 23$ cases and 24/34 controls. There were more patients with cystic fibrosis and bronchiectasis with ARIs (8/ 23) than with ASIs (3/34; not statistically significant). There was no difference in non-respiratory coexistent disease including malignancy. A history of cigarette smoking was less common with ARIs (14/23 compared with $29 / 34$, odds ratio $0 \cdot 12$; CI 0.02 to $0 \cdot 75$ ). More patients with ARIs had received antibiotics $(p=0.003)$, and particularly aminopenicillins $(p=0.0002)$, in the previous month. Coexisting pathogens were cultured from sputum with more ARIs $(5 \times S$ pneumoniae, $2 \times S$ aureus, $1 \times P$ s aeruginosa) than with ASIs $(3 \times S$ pneumoniae $1 \times M$ catarrhalis, $\mathrm{p}=0.038$ ). There was no difference in outcome between the two groups as judged by duration of hospital stay or death rate at three and 12 months. ARIs were associated with mixed infections, greater antibiotic exposure in the preceding month, particularly to aminopenicillins and chronic suppurative lung disease. [Assistance acknowledged from Glaxo.]

Cefaclor MR versus co-amoxiclav in the treatment of acute exacerbations of chronic bronchitis in general practice

J HOSIE, C LANGAN, G LINDSAY, D ROBERTS, G WILSON Department of Bacteriology, Southern General Hospital, Glasgow; Lilly Industries Ltd, Basingstoke Most antibiotics for the treatment of acute exacerbations of chronic bronchitis are given for seven days, although there is a trend towards five days of treatment. The common view is that patients discontinue treatment when they feel better. We describe a study where patients were allowed to take antibiotics for up to 14 days and discontinue at their own discretion. A total of 223 patients (125 men) presenting with acute exacerbation of chronic bronchitis were randomly assigned to either cefaclor MR twice daily or coamoxiclav three times per day. Each patient was instructed to continue their treatment until they felt better. Only patients treated for more than three days were included in the analysis (112 men). Seventy cefaclor MR patients were classed as cured by their GP and 86 coamoxiclav patients. Duration of treatment of cured patients was not significantly different, with cefaclor MR being taken for 11.4 (3.1) days and co-amoxiclav for 10.7 (3.5) days. Six cefaclor MR patients $(5.9 \%)$ withdrew from the study due to adverse events compared with $13(12.9 \%)$ of the co-amoxiclav patients $(p=0.092)$. Patients classed as cured were interviewed one month after cessation of treatment. There was no significant difference between the rate of relapse in either treatment group. This study suggests that, contrary to popular belief, patients may require longer courses of therapy than the commonly prescribed seven or five days for their symptoms to be reduced to their satisfaction.

Impact of bronchoalveolar lavage in suspected pneumonia after renal transplantation

MJ IREDALE, SA EVANS, C BELL, MA WOODHEAD Department of Respiratory Medicine, Manchester Royal Infirmary, Manchester Bronchoalveolar lavage (BAL) is an effective means of diagnosing pulmonary infection in patients after renal transplantation. The impact of BAL on outcome in this group of patients has seldom been evaluated. We retrospectively studied all patients who had undergone BAL for suspected pneumonia after renal transplantation from January 1989 to August 1994 to evaluate whether BAL influenced therapy and/or survival. Twenty six lavages were performed on 25 patients (mean age 46 years, median time from transplant $2 \cdot 8$ months). All patients were treated with immunosuppressive agents: 21 cyclosporin, 24 steroids, 13 azathioprine, and five antithymoctye globulin or equivalent at the time of bronchoscopy. Eighteen (69\%) lavages were positive; seven for $P$ carinii, seven for cytomegalovirus, three for fungi, two for $M$ tuberculosis, and two for influenza A. Ten of the 18 patients with a positive BAL survived the acute episode compared with seven of eight patients with a negative BAL $(p=0 \cdot 11)$. Fifteen of the 18 patients with a positive BAL had an abnormal chest radiograph compared with one of the eight patients with a negative BAL $(p<0.001)$. BAL findings resulted in a change in treatment in 14 cases. In 10 cases specific treatment was started. Eight $(80 \%)$ of these patients survived. In four cases treatment was stopped. In four cases the BAL result led to continuation of previous empirical therapy, two (50\%) of these patients survived. All patients who survived the acute episode are still alive. Median follow up for those surviving the acute episode is 20 months. In conclusion, BAL leads to a change of treatment, or confirms empirical treatment, in the majority of patients studied with beneficial effects on survival.

Comparison of pentamidine absorption in the isolated perfused rabbit lung when administered by intratracheal instillation and ultrasonic nebuliser

A BELlo, AJ HUTT, c MARRIOTT Department of Pharmacy, King's College London Pentamidine isethionate (PI) has been commonly administered as an aerosol for the treatment of pulmonary infection by Pneumocystis carinii. However, little is known regarding the effect of this delivery method on PI absorption from the lung. Using the recirculating isolated perfused rabbit lung (RIPRL) we have investigated the absorption of PI administered by intratracheal instillation (ITI) and ultrasonic nebuliser (UN). PI was administered at $0.5,1.0$ and $2.0 \mathrm{mg}$ doses to the RIPRL by ITI and UN. The mean particle size of the aerosol was determined to be $4.0(0 \cdot 1) \mu \mathrm{m}$. Following administration concentrations of PI in perfusate were determined by HPLC. Delivery by both methods resulted in dose related increases in maximal perfusate concentration (Cmax) and area under the perfusate concentration time curve (AUC) (table).

\begin{tabular}{|c|c|c|c|c|}
\hline \multirow[t]{2}{*}{ Parameter } & \multirow[t]{2}{*}{ Method } & \multicolumn{3}{|l|}{ Dose (mg) } \\
\hline & & 0.5 & $1 \cdot 0$ & $2 \cdot 0$ \\
\hline$\underset{(\mu \mathrm{g} / \mathrm{ml})}{\mathrm{Cmax}}$ & $\begin{array}{l}\text { ITI } \\
\text { UN }\end{array}$ & $\begin{array}{l}1.5(0.3) \\
3.3(0.3)^{*}\end{array}$ & $\begin{array}{l}4.3(0.8) \\
6.9(1.5)\end{array}$ & $\begin{array}{r}9.2(1.4) \\
15.5(0.5)^{*}\end{array}$ \\
\hline$\underset{(\mu \mathrm{g} / \mathrm{l} . \mathrm{h})}{\mathrm{AUC}}$ & $\begin{array}{l}\text { ITI } \\
\text { UN }\end{array}$ & $\begin{array}{l}2 \cdot 1(0 \cdot 6) \\
5 \cdot 7(0 \cdot 7)^{*}\end{array}$ & $\begin{array}{l}5 \cdot 7(1.1) \\
7 \cdot 5(1.5)\end{array}$ & $\begin{array}{l}12 \cdot 3(0 \cdot 8) \\
21 \cdot 7(2 \cdot 1)^{*}\end{array}$ \\
\hline
\end{tabular}

Data are mean (SD), $\mathrm{n}=3 .{ }^{*} \mathrm{p}<0.01$ (Student's $t$ test).

Comparison of ITI with the UN delivery showed increases in Cmax ( 1.6 to 2.2 fold) and AUC ( 1.3 to 2.7 fold). At the 1.0 and $2.0 \mathrm{mg}$ doses there was also a significant increase $(p<0.05)$ in the Tmax values following UN administration. The increased absorption following administration by UN may be due to increased exposure of the nebulised dose to the more absorptive, highly perfused surfaces of the lung.

Effect of nebulised recombinant DNase on mediators of inflammation in cystic fibrosis

C costello, J hayes, C O'Connor, mX Fitzgerald Department of Medicine, University College Dublin; St Vincent's Hospital, Dublin DNA released by degenerating neutrophils is a significant contributor to airway mucous plugging in cystic fibrosis (CF). The connection between hyperviscous mucous, opportunistic bacterial infection and inflammation suggests that antimucolytic agents, in addition to reducing airway obstruction, may also attenuate the infection/in- 
flammation cycle which leads to lung destruction in CF. Recently, nebulised recombinant DNase has been introduced as an antimucolytic therapy in CF and initial studies indicate that it may be effective in reducing the number of acute infectious episodes experienced by patients. The aim of this study was to examine whether DNase therapy might also reduce the chronic neutrophil-dominated inflammation which underlies lung disease in CF. Blood and sputum samples were collected from $15 \mathrm{CF}$ patients $(8 \mathrm{~F}, 7 \mathrm{M})$ prior to initiation of nebulised DNase therapy and at six and 12 weeks thereafter. At entry to the study all patients displayed chronic pulmonary bacterial colonisation but none were acutely infected. Plasma samples were analysed for interleukin-8 (IL-8), $\alpha_{1}$-protease inhibitor $\left(\alpha_{1} \mathrm{Pl}\right)$, and neutrophil elastase in complex with $\alpha_{1} \mathrm{Pl}(\mathrm{NE}-\mathrm{Pl})$. Sputum samples were assessed for IL-8, $\alpha_{1} \mathrm{Pl}$, and levels of active neutrophil elastase (NE). Initial plasma levels of IL-8 and $\alpha_{1} \mathrm{Pl}$ were elevated above those observed in normal controls $(p<0.001)$, indicating underlying inflammation. Twelve weeks of DNase therapy failed to reduce plasma levels of either IL-8 or $\alpha_{1} \mathrm{Pl}$. A significant decrease in sputum levels of $\alpha_{1} \mathrm{Pl}$ was observed over the 12 week treatment period (pretreatment: $35.6(6.7) \mathrm{mg} / \mathrm{ml}$; after treatment: $8.75(3.0) \mathrm{mg} / \mathrm{ml}$, $\mathrm{p}<0.002)$. This was not accompanied by a similar decrease in sputum levels of either IL-8 or NE activity, although a downward trend in NE activity was noted (pretreatment: $93.8(12 \cdot 2)$ units; after treatment: $70 \cdot 0(8 \cdot 5)$ uunits, $p=0.058)$. These results indicate that short term treatment with nebulised DNase has little effect in attenuating underlying inflammation in CF. [This work was supported by the Cystic Fibrosis Society of Ireland.]

Empyema of the thorax: a four year experience from Pakistan

J KHAN, S MOHSIN, I QURESHI, F HUSSAIN Aga Khan University Hospital, Karachi, Pakistan Empyema thoracis continues to be a common and troublesome clinical problem with significant morbidity and mortality worldwide. The aetiology, organisms involved, concomitant diseases and prognosis regarding empyema differ from series to series. There is a difference of opinion regarding its management. The aetiologies, microbiological findings and management of 81 patients of empyema were analysed. The patients were, on average, 36.4 years old; $64 \%$ had underlying diseases which included diabetes mellitus $(19 \%)$, bronchial asthma (9\%), and hypertension ( $7 \cdot 4 \%)$. In $62 \%$ of patients empyema developed secondary to a bronchopulmonary infection. Other aetiologies were iatrogenic $(11 \%)$, infradiaphragmatic sepsis $(8 \cdot 6 \%)$, idiopathic $(23 \cdot 5 \%)$. Cultures were positive in $66.6 \%$ cases. Among the culture positive cases a single bacteria was isolated in $74 \%$. Multiple organisms grew on the remaining $26 \%$ positive cultures. Mycobacterium tuberculosis was isolated from $8.6 \%$ of cases. Aerobes were isolated from $92.6 \%$ of positive cultures and anaerobes from $7 \cdot 4 \%$ of the remaining positive cultures. Diagnostic thoracocentesis was done on all patients and subsequently intercostal chest tube drainage was required in $57 \%$ of patients and more aggressive surgery was performed on $22 \%$ of patients; $16 \%$ of patients were treated with serial thoracocentesis and $5 \%$ received antibiotics only. Although microbiological evidence of $M$ tuberculosis was available in seven cases, $36.7 \%$ of patients were given antituberculous therapy because of clinical suspicion of tuberculosis. The length of the hospitalisation averaged 13 days. $12 \%$ patients died, the rest recovered; $8.6 \%$ of deaths came about as a direct result of empyema. The analysis of this case series suggests that, in developing countries like Pakistan, bronchopulmonary infection is the greater cause of empyema among which tuberculosis stands out. Case fatality ratio is very high so there is a need for early detection and selection of appropriate antibiotics combined with an immediate drainage procedure.

Effect of a single nebulised dose of frusemide on cough induced by low chloride solution in patients with chronic dry cough of unknown aetiology

UG LALLOO, M WORSDELL, DM SCOTT, PJ BARNES, KF CHUNG Department of Thoracic Medicine, National Heart and Lung Institute and Royal Brompton Hospital, London Nebulised frusemide inhibits cough induced by low chloride content solutions (LCC) in healthy subjects. We examined the effects of a single nebulised dose of frusemide on cough induced by LCC in 10 selected patients (eight women) of mean (SD) age 53.2 (11.4) years with a chronic dry cough of unknown aetiology in a double blind placebo (saline) controlled crossover design study. These patients had a cough for $>2$ years and were fully investigated for a cause for the cough and had failed to respond to a course of high dose inhaled steroids and had an enhanced cough response to LCC. None were on regular medications for cough and all antitussives were stopped 24 hours prior to the study. Frusemide $(3.75 \mathrm{mg} / \mathrm{ml}$ in saline) or saline was administered for 8 minutes via a De Vilbiss nebuliser with an output of $1 \mathrm{ml} / \mathrm{min}$. Prior to each of the two study days, which were 1 week apart, the patients had an LCC cough challenge to determine the highest LCC which produced eight or more coughs. The LCC challenge was repeated at 20,120 , and 240 minutes following frusemide or saline administration. The baseline coughs were reproducible $(p=0 \cdot 37)$. Frusemide had a weak effect on the cough response to LCC at 20 minutes $(p=0.09)$ and 240 minutes $(p=0.09)$ compared with saline $(p=0.10$ and $p=$ 0.64 respectively) (Wilcoxon ranks of paired individual data). Four patients showed a significant response to frusemide which was sustained up to 240 minutes $(p=0 \cdot 006)$. The weak response could be explained by hyperalgesia of the peripheral cough receptors in this select group of patients and warrants further study with chronic dosing. There is also a subgroup of "responders" who may benefit from long term frusemide.

Capsaicin cough challenge: relationship to airways obstruction and reactivity in asthma

mJ Doherty, pMa Calverley, mg pearson Aintree Chest Centre, Liverpool The capsaicin cough challenge test has previously been used to assess the sensitivity of the cough reflex in humans. As cough is often a troublesome symptom in asthma, we postulated that this measure of the cough reflex may relate to the severity of the disease. The measures of disease severity used were peak flow variability, \% predicted $\mathrm{FEV}_{1}$, reported symptoms of cough, and the degree of nonspecific bronchial hyperreactivity. We studied 43 asthmatics $(27$ men, mean age 49 years, range $22-73$, mean (SE) \% predicted $\mathrm{FEV}_{1} 73$ (3)\%, mean \% predicted FVC 87 (4)\%). Thirty four subjects kept a record of their peak flow and daily symptoms of cough for two weeks; 27 complained of cough on more than half the days. All 43 subjects had spirometry before a capsaicin cough test which was performed using a single inhalation technique and nine increasing concentrations of capsaicin from 1.95 to $500 \mu \mathrm{M}$. The concentration at which the subject coughed five times was noted (C5). Ten minutes after the cough test and after checking that the $\mathrm{FEV}_{1}$ had returned to baseline, we performed a histamine challenge test. The 34 subjects who kept the diary card did not differ in any variable from the remaining nine subjects. The median $\mathrm{C} 5$ response was the sixth concentration whilst the geometric mean lay between the fourth and fifth concentrations; this is significantly lower than normal subjects in whom the median is above the ninth concentration. The median and geometric mean $\mathrm{PC}_{20}$ for histamine were $1(0.46) \mu \mathrm{M}$ and the mean peak flow variability was $15(1 \cdot 36) \%$. Although both $\mathrm{C} 5$ and histamine $\mathrm{PC}_{20}$ were reduced we found no correlation between capsaicin sensitivity (C5) and \% predicted $\mathrm{FEV}_{1}$ (Pearson's $\left.r=-0.08\right)$, peak flow variability $(r=0.05)$, or $\mathrm{PC}_{20}$ for histamine (Spearman's $r=0 \cdot 23$ ). There was a weak relationship between sensitivity to capsaicin and reported symptoms of cough analysed non-parametrically $(r=-0.33)$. In conclusion, we found no evidence that the sensitivity of the cough reflex as measured by capsaicin was related to airway calibre or bronchial hyperreactivity, suggesting that different mechanisms may be involved in the enhanced cough response of asthmatics.

Bronchodilator responsiveness of impulse oscillation model parameters in healthy and asthmatic subjects

D MACLEOD, P REINHOLD, H SMITH, J PRICHARD University Medicine, Southampton General Hospital, Southampton Oscillometry examines the behaviour of the total respiratory system with time and frequency. Using the new Impulse Oscillation System (IOS, Jaeger, Germany) mechanical function was examined in six asthmatic (A) (mean (SD) age 42 (18) years, mean $\mathrm{FEV}_{1} \%$ predicted $58(17) \%$ ) and nine healthy (H) subjects (mean age 37 (13) years) undergoing bronchodilator challenge (salbutamol, volumatic device). The impulse length was 0.1 seconds with generation every 0.2 seconds and real and reactive parts of the impedance were determined between 5 and $35 \mathrm{~Hz}$. Using these data, a seven element modified Mead model was fitted to calculate central and peripheral resistance ( $R z$ and $R p)$, total resistance $(\mathrm{Rrs})$, lung and bronchial compliance $(\mathrm{Cl}$ and $\mathrm{Cb})$, and central airway inertance (Lz). The results are shown in the table in $\mathrm{kPa} / \mathrm{l} / \mathrm{s}$ 


\begin{tabular}{lllllll}
\hline & $R z$ & $R p$ & $R r s$ & $C l$ & $C b$ & $L z$ \\
\hline H (pre) & 0.14 & 0.106 & 0.246 & 0.006 & 0.0005 & 0.001 \\
H (post) & 0.11 & 0.083 & 0.193 & 0.006 & 0.0004 & 0.001 \\
\% change & -21 & -22 & -22 & 0 & -20 & 0 \\
A (pre) & 0.096 & 0.672 & 0.768 & 0.0045 & 0.00012 & 0.0016 \\
A (post) & 0.086 & 0.472 & 0.558 & 0.0058 & 0.00023 & 0.0014 \\
$\%$ change & -10 & -30 & -27 & +29 & +92 & -13 \\
\hline
\end{tabular}

throughput decreased by $88 \%$ and staff time required by $84 \%$; (4) an initial software fault suspended the study. Later hardware power faults caused problems. New versions appear to have overcome these. We conclude that (1) ED offers potential benefits of improved data quality, faster data throughput, and results in possible cost savings; (2) there is a high degree of patient acceptibility for the ED.

( $\%$ change $=$ absolute change $/$ baseline value $)$. Oscillometry is an effortindependent respiratory function test. Our bronchial compliance and peripheral resistance findings are of particular interest. More critical clinical work is needed, however, to explore test signal influences and modelling validity.

Perception of asthma and breathlessness during exercise scored on a visual analogue scale

G ERHABOR, AH KENDRICK, EC SMITH, G LASZLO Respiratory Department, Bristol Royal Infirmary, Bristol We wished to determine whether asthmatics could distinguish between normal breathlessness and the sensation of asthma during exercise using a $100 \mathrm{~mm}$ visual analogue scale (VAS). 11 asthmatics (mean age 26.1 (2.9) years) with exercise induced asthma and 10 normal subjects (mean age $26 \cdot 5(5 \cdot 3)$ years) were studied. Each performed a Balke treadmill test at $5.5 \mathrm{kph}$ with a one minute incremental grade of $2 \%$ /minute up to $20 \%$, inspiring dry air at room temperature. Expired minute ventilation (VE) was averaged over the last 30 seconds of each minute. Prior to each increase in grade the subjects quantified their sensation of breathlessness (SoB) and asthma using the VAS (Harty et al. Clin Sci 1993;85:229-36). The relation of VAS to VE was obtained by linear regression analysis. VAS $(\mathrm{mm})$ at a $\dot{V E}$ of $50 \mathrm{l} / \mathrm{min}\left(\mathrm{VAS}_{50}\right)$ and at $60 \%$ maximum $\dot{V E}$ $\left(\mathrm{VAS}_{60}\right)$ were calculated. All subjects achieved a cardiac frequency of $>80 \%$ maximum predicted (range $82-104 \%$ ). For all subjects there were significant linear relationships of VAS to $\mathrm{VE}$ for SoB and asthma. The results are given in the table as mean (range). All indices were

\begin{tabular}{llll}
\hline & \multicolumn{1}{l}{ Asthmatics } & \multicolumn{1}{l}{ Controls } \\
\cline { 2 - 3 } & \multicolumn{1}{l}{ Asthma } & \multicolumn{1}{c}{ SoB } & \\
\hline Slope & $0 \cdot 6(0 \cdot 12-1 \cdot 3)$ & $0.96(0 \cdot 46-1 \cdot 8)$ & $0 \cdot 9(0 \cdot 5-1 \cdot 4)$ \\
VAS $_{50}$ & $19 \cdot 1(4 \cdot 6-38 \cdot 3)$ & $31 \cdot 3(13 \cdot 9-56 \cdot 4)$ & $29 \cdot 2(14 \cdot 5-49 \cdot 4)$ \\
VAS $_{60}$ & $16.9(3 \cdot 6-42 \cdot 5)$ & $27.6(9 \cdot 7-61 \cdot 2)$ & $22 \cdot 7(4 \cdot 3-45 \cdot 4)$ \\
\hline
\end{tabular}

significantly different $(p<0.05)$ when comparing the sensations of breathlessness and of asthma during exercise in the asthmatic subjects. We conclude that asthmatics can distinguish the sensation of asthma from that of normal breathlessness.

Comparison of two methods of collecting respiratory diary card data: conventional paper and electronic diary

FGE BÖLLERT, SP MATUSIEWICZ, M DEWAR, B TIPLADY, M MITCHELL, JA INNES, AP GREENING, GK CROMPTON Respiratory Unit, Western General Hospital; Astra Clinical Research Unit The study compared the suitability of conventional paper and electronic diaries (Apple Newton Message Pad with customised software) in collecting respiratory trial data. An open randomised crossover study was performed in 22 patients (13 men, age 18-70) with airflow obstruction who recorded twice daily peak flow measurements, asthma symptoms, and rescue inhaler usage in a paper (PD) and an electronic (ED) diary for one month each. Variables assessed were (1) completeness, timeliness and quality of data; (2) preferences of patients and staff for PD or ED; (3) differences in workload and costs; (4) speed of data throughput. Data were analysed using a standard parametric method for two period crossover after arcsine transformation and descriptive statistics. The results showed that (1) data reliability improved although there were more missing data with ED than PD $(p=0.0001)$ due partly to a decision not to allow retrospective data entry on ED; (2) $59 \%$ of patients preferred the ED and $18 \%$ the PD. Age, gender and familiarity with technology had no marked association with the preference. Both ED and PD were judged easy to use; (3) days needed for data

Respiratory observations on trekkers accompanying the 40th anniversary British Everest Expedition

PL JONES, A PEACOCK, R EDGE Department of Anaesthetics, University Hospital of Wales, Cardiff; Department of Respiratory Medicine, Western Infirmary, Glasgow; Newcastle Medical School Little is known of the respiratory parameters of subjects as they acclimatise to altitude. We have measured $\mathrm{FVC}, \mathrm{SpO}_{2}$ and $\mathrm{PAO}_{2}$ on 39 trekkers as they walked from Jiri $(1860 \mathrm{~m})$ to Base Camp Everest $(5550 \mathrm{~m})$ over a three week period. $\mathrm{PambO}_{2}$ was predicted from known altitudes (Kuntz et al. 1906; West et al. 1983) and used to calibrate a fuel cell which

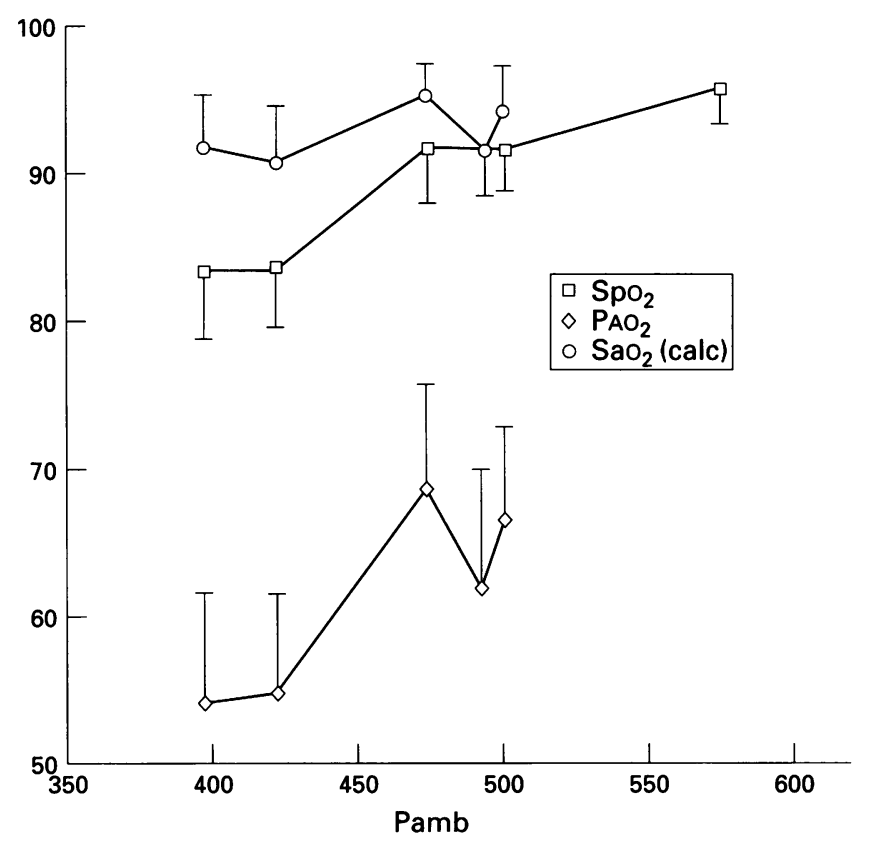

measured $\mathrm{PAO}_{2}$ following a maximum expiratory manoeuvre. Percutaneous oxygen saturation $\left(\mathrm{SpO}_{2}\right)$ was measured using a pulse oximeter (PneuPAC). Oxygen saturation $\left(\mathrm{SaO}_{2}(\mathrm{calc})\right)$ was also predicted assuming $\mathrm{PaO}_{2}$ equal to the $\mathrm{PAO}_{2}$ using an algorithm (Mohan et al. 1977) in which predicted $\mathrm{PaCO}_{2}(\mathrm{RQ}=0.85)$ and arterial $\mathrm{pH}$ are factors. It has been shown that $\mathrm{pH}$ changes little below $5000 \mathrm{~m}$ (Dill et al. 1937; West et al. 1962). The results demonstrate the progress of acclimatisation in the face of increasing altitude. The observed $\mathrm{Spo}_{2}$ was increasingly unstable above $3440 \mathrm{~m}$. This suggests instability either of ventilation or of $\mathrm{V} / \mathrm{Q}$ matching. The discrepancy between measured $\mathrm{Spo}_{2}$ and predicted $\mathrm{SaO}_{2}$ (calc) $(\mathrm{p}<0.0001$ for the higher altitudes) may indicate a deterioration in pulmonary function caused by pulmonary oedema.

Comparison of respiratory function after single lung transplantation for fibrosing alveolitis and emphysema

RA CHACON, TN STONE, PA CORRIS, JH DARK, GJ GIBSON Departments of Respiratory Medicine and Cardiothoracic Surgery, Freeman Hospital, Newcastle upon Tyne Single lung transplantation (SLT) is performed in patients with both advanced fibrosing alveolitis (CFA) and emphysema (E). We have compared 10 patients receiving SLT for E with six receiving SLT for CFA to assess how the native lung influences tests of overall respiratory function. Patients were studied for a mean of 17 months (range 6-48) after SLT when free of complications. None had had evidence of obliterative bronchiolitis. Investigations included lung volumes (by plethysmography), spirometry, MEFV curves (VEmax and moments analysis), TLCO, and KCO and quantitative $\dot{V} / Q$ scans using ${ }^{133} \mathrm{Xe}$ and ${ }^{99 \mathrm{~m}} \mathrm{Tc}$ respectively. Relative $\dot{\mathrm{V}}$ was 
measured after a single full inspiration. The results are shown in the tables. In comparison with patients receiving SLT for CFA, those

Table 1 Mean (SE) lung volumes, CO transfer and scans

\begin{tabular}{|c|c|c|c|c|c|c|}
\hline & $\begin{array}{l}\text { TLC } \\
\text { (\% of reci }\end{array}$ & $\begin{array}{l}V C \\
\text { ient predicted }\end{array}$ & $\begin{array}{l}T_{L C O} \\
\text { values) }\end{array}$ & $\mathrm{KCO}_{\mathrm{C}}$ & $\begin{array}{l}\text { Graft } \dot{Q} \\
(\%)\end{array}$ & $\begin{array}{l}\text { Graft } \dot{V} \\
(\%)\end{array}$ \\
\hline CFA & $\begin{array}{l}103 \\
(11 \cdot 4)\end{array}$ & $\begin{array}{l}76 \cdot 3 \\
(5 \cdot 5)\end{array}$ & $\begin{array}{l}56 \cdot 7 \\
(5 \cdot 6)\end{array}$ & $\begin{array}{c}88 \cdot 2 \\
(10 \cdot 5)\end{array}$ & $\begin{array}{l}74 \cdot 8 \\
(2 \cdot 9)\end{array}$ & $\begin{array}{l}62 \cdot 7 \\
(3 \cdot 5)\end{array}$ \\
\hline $\mathrm{E}$ & $\begin{array}{l}140 \\
(5 \cdot 0)\end{array}$ & $\begin{array}{l}76 \cdot 1 \\
(5 \cdot 5)\end{array}$ & $\begin{array}{l}75.9 \\
(4.9)\end{array}$ & $\begin{array}{l}112 \\
(4 \cdot 6)\end{array}$ & $\begin{array}{l}79 \cdot 4 \\
(2 \cdot 4)\end{array}$ & $\begin{array}{l}86 \cdot 8 \\
(2 \cdot 2)\end{array}$ \\
\hline$p$ & 0.024 & NS & 0.026 & NS & NS & 0.0002 \\
\hline
\end{tabular}

Table 2 Mean (SE) MEFV curves

\begin{tabular}{|c|c|c|c|c|c|c|}
\hline & \multicolumn{3}{|l|}{ VEmax } & \multicolumn{3}{|c|}{ Moments } \\
\hline & $\begin{array}{l}75 \% \text { VC } \\
\text { (\% of recipi }\end{array}$ & $\begin{array}{l}50 \% V C \\
\text { ient predicted }\end{array}$ & $\begin{array}{l}25 \% V C \\
\text { d values) }\end{array}$ & $\begin{array}{l}1 \\
(s)\end{array}$ & $\begin{array}{l}2 \\
\left(s^{2}\right)\end{array}$ & $\begin{array}{l}3 \\
\left(s^{3}\right)\end{array}$ \\
\hline CFA & $\begin{array}{l}84.0 \\
(9 \cdot 2)\end{array}$ & $\begin{array}{l}57 \cdot 0 \\
(10 \cdot 3)\end{array}$ & $46 \cdot 8$ & $\begin{array}{l}0.72 \\
(0.06)\end{array}$ & $\begin{array}{l}1 \cdot 07 \\
(0 \cdot 2)\end{array}$ & 2.66 \\
\hline $\mathrm{E}$ & $\begin{array}{l}56 \cdot 5 \\
(7 \cdot 1)\end{array}$ & $\begin{array}{l}40 \cdot 5 \\
(5 \cdot 4)\end{array}$ & $\begin{array}{l}34 \cdot 2 \\
(9 \cdot 1)\end{array}$ & $\begin{array}{l}0.94 \\
(0.07)\end{array}$ & $\begin{array}{l}2 \cdot 25 \\
(0 \cdot 36)\end{array}$ & $\begin{array}{l}7.95 \\
(1.62)\end{array}$ \\
\hline$p$ & 0.04 & NS & NS & 0.035 & 0.013 & 0.014 \\
\hline
\end{tabular}

with emphysema have similar perfusion but relatively more ventilation of the transplanted lung and better overall $\mathrm{CO}$ transfer, despite generally worse airway function. The larger TLC and MEFV moments are attributable to the volume and very slow emptying of the native emphysematous lung. Tests of airway function are likely to be less sensitive for detecting complications after SLT for emphysema compared with CFA.

Time constants during tidal expiration and maximally forced expiration

F DENBY, RG MADGWICK, MJ MORRIS Osler Chest Unit, Churchill Hospital, Oxford From a maximally forced flow volume curve from total lung capacity (TLC) the time constant, trs, of the respiratory system can be calculated as the ratio of volume/flow. It has been shown that the time constant of a fully relaxed expiration from TLC is not different from that of a maximally forced expiration. We have postulated that the time constant of the respiratory system can be estimated from analysis of the last part of tidal expiration when relaxation of inspiratory muscles has occurred. The aim of this study was to compare time constants measured from tidal expiration, trsTID, in normal subjects and in patients with airflow obstruction (AFO) with the values obtained from maximally forced flow volume curves with volume measured (a) by integrating flow at the mouth, trsMO, and (b) at the chest wall using transmural body plethysmography, trs $\mathrm{CH}$. In 10 normal subjects mean (SD) $\mathrm{FEV}_{1} \%$ predicted was 120 (16) and in 11 patients with AFO FEV $1 \%$ predicted was 48 (21). There was a significant difference between the normal subjects and the AFO patients in the time constants measured by each of the three methods.

\begin{tabular}{llll}
\hline & $\operatorname{trs} T I D(s)$ & $\operatorname{trs} M O(s)$ & $\operatorname{trs} C H(s)$ \\
\hline Normal subjects & $0.45(0.07)$ & $0.48(0.13)$ & $0.41(0.11)$ \\
AFO patients & $1.5(1.04)$ & $3.1(3.3)$ & $0.9(0.41)$ \\
\hline
\end{tabular}

In all the subjects the difference between trsMO and $\operatorname{trs} \mathrm{CH}$ was significantly related to $\mathrm{dv}$, the maximum volume of alveolar gas compression, difference $=-1.55+4.3 \mathrm{dv}, r^{2}=0.57, \mathrm{p}<0.001$. In the normal subjects there was no important difference between trs measured in all three ways; however, in the AFO patients trsTID was closer to trs $\mathrm{CH}$ than to trsMO, probably because alveolar gas compression is not occurring during tidal expiration.

Evaluation of an index of overinflation derived from analysis of tidal breathing

E GRINT, RG MADGWICK, MJ MORRIS Osler Chest Unit, Churchill Hospital, Oxford An index of overinflation (EV) can be derived from tidal flow-time or flow-volume tracings (Morris et al. ERF 1990;3: 901-9). The aim of this study was to assess further the validity of this measurement. During tidal breathing EV was measured in two groups of subjects: (1) seven normal subjects in whom, during relaxed tidal breathing, two expiratory resistances of increasing severity were added. The resulting changes in end expiratory tidal volume (EEV) were measured by pneumotachography. Paired $t$ tests showed that there was no difference between the EEV increments measured by the two methods (low resistance - spirometric volume increment $(\mathrm{SVI})=0.271($ mean $(\mathrm{SD})), \mathrm{EV} 0.23(0.08) \mathrm{l}$; higher resistance $-\mathrm{SVI}$ $0.65(0.18) 1$, EV $0.52(0 \cdot 19)$ l). Regression analysis showed that, with the lower resistance, SVI was significantly related to $\mathrm{EV}, \mathrm{SVI}=$ $0.003+1.15 \mathrm{EV}, r^{2}=0.77, \mathrm{p}<0.01$. With the higher resistance the regression relationship between these two variables was not significant. (2) Seventy nine patients with a clinical diagnosis of airflow obstruction (AFO) in whom thoracic gas volume was measured by constant volume plethysmography. In this group of patients (FRC \% predicted 153 (44)), EV predicted the degree of overinflation according to the equation, FRC $\%$ predicted $=110+149 \mathrm{EV}, r^{2}=0.46, \mathrm{p}<0.001$. It is hypothesised that $\mathrm{EV}$ measures the volume that $\mathrm{EEV}$ is above the relaxation volume of the respiratory system for that part of the lung with open airways over the tidal volume range, and thus does not include the volume increment caused by trapped gas.

Increased QT dispersion: a novel marker of abnormal myocardial repolarisation during acute hypoxaemia

DG KIELY, RI CARGILL, AD STRUTHERS, BJ LIPWORTH Department of Clinical Pharmacology, Ninewells Hospital and Medical School, Dundee Prolongation of QT interval has been associated with cardiac dysrhythmias and sudden death, reflecting abnormal myocardial repolarisation. Hypoxaemia and $\beta$ agonists have both previously been shown to increase QT interval, raising the possibility of deleterious synergistic effects in acute severe asthma. Recently QT dispersion (interlead variability in QT interval) has been proposed as being more sensitive than QT interval as a marker of repolarisation abnormalities. We have therefore evaluated the effects of acute hypoxaemia on both QT interval and QT dispersion in eight normal men. After resting to achieve baseline haemodynamics, subjects were rendered hypoxaemic for 30 minutes by breathing an $\mathrm{N}_{2} / \mathrm{O}_{2}$ mixture to achieve $\mathrm{SaO}_{2}$ of 75-80\%. From the ECG, lead II corrected QT interval (QTc) and overall corrected QT dispersion were measured using a computer linked digitising tablet according to standard criteria. QTc dispersion was significantly increased during hypoxaemia compared with baseline at 69 (6) $v 50$ (5) ms respectively $(\mathrm{p}<0.05), 95 \%$ CI for mean difference $2-35 \mathrm{~ms}$, whilst QTc interval was not significantly affected by hypoxaemia; 428 (8) $v 416(10) \mathrm{ms}(\mathrm{p}=0 \cdot 3)$. This was not associated with any change in serum potassium: $4.07(0.06) v 4.06$ $(0.07) \mathrm{mmol} / \mathrm{l} \quad(p=0.62)$. Plasma catecholamines were not significantly affected by hypoxaemia: noradrenaline $4.75(0.65) v 3.97$ $(0.36) \mathrm{nmol} / \mathrm{l}(\mathrm{p}=0.11)$, adrenaline $0.22(0.027) v 0.16(0.016) \mathrm{nmol} /$ $1(p=0 \cdot 17)$. There were significant increases in both heart rate (HR) and mean pulmonary arterial pressure (MPAP) in response to hypoxaemia; HR $78(3.4) v 64(1.9) \mathrm{bpm}(\mathrm{p}<0.05)$ and MPAP $23(1.2)$ $v 9(0.9) \mathrm{mmHg}(\mathrm{p}<0.05)$. In summary, QTc dispersion was found to be a more sensitive marker of repolarisation abnormalities than QTc interval during acute hypoxaemia. Furthermore, significant increases in QTc dispersion cannot be explained by changes in circulating catecholamines. Thus, measurement of QTc dispersion may be a useful clinical tool for predicting patients at risk of hypoxaemia associated arrhythmias.

Placebo controlled study comparing budesonide $256 \mu \mathrm{g}$ and $128 \mu \mathrm{g}$ with fluticasone propionate $200 \mu \mathrm{g}$ in patients with seasonal allergic rhinitis

MA STERN, R DAHL, C SCHREWELIUS Midlands Asthma and Allergy Research Association (MAARA), Leicester General Hospital, Leicester, UK; Aarhus Kommunehospital, 8000 Aarhus C, Denmark; Astra Draco $A B$, Clinical Research and Development, Lund, Sweden In a two centre study in the UK and Denmark 602 patients ( $\geqslant 18$ years) were evaluated (according to all patient treated analysis) to compare the efficacy and safety of budesonide aqueous nasal spray $256 \mu \mathrm{g}$ (BUD256), $128 \mu \mathrm{g}$ (BUD128), placebo and fluticasone propionate aqueous nasal spray $200 \mu \mathrm{g}$ (FP200), all once daily, in the treatment of seasonal allergic rhinitis. We used a parallel, randomised design, double blind for BUD and placebo and single blind to the investigator 
for FP. A one week run in period preceded trial medication for 4-6 weeks during the grass pollen season. Patients recorded nasal symptoms on a diary card. All active treatments produced significantly lower mean scores than did placebo for all nasal symptoms $(p<0.05)$. BUD256 was significantly better than FP200 in reducing sneezing $(p<0 \cdot 05)$. We found no differences in effects between BUD128 and FP200. Adverse events recorded during study were mild and transient for each treatment group. The results suggest that the clinical effect of BUD128 and FP in the nose is similar and that BUD256 is more effective in seasonal allergic rhinitis than FP200.

Study to compare the efficacy of budesonide (Pulmicort ${ }^{\circledR}$ Turbohaler ${ }^{\circledR}$ and fluticasone propionate (Flixotide ${ }^{\circledR}$ Diskhaler ${ }^{\circledR}$ ) in the treatment of asthma

G BASRAN, R SCOTT, M CAMPBELL, A KNOX, R SMITH, J VERNON, A WADE FOR A UK STUDY WORKING GROUP Rotherham District Hospital, Moorgate Road, Rotherham The study objective was to compare the efficacy of budesonide Turbohaler (Bud TBH) with that of fluticasone propionate Diskhaler (FP DH), at half the baseline dose, in asthmatic patients requiring daily doses of 400 or $800 \mu \mathrm{g}$ of conventional inhaled steroids. Only patients with symptomatic asthma during run in were randomised. The study was of a multicentre, open, parallel group design with treatment for eight weeks with Bud TBH or FP DH at doses of 100 or $200 \mu \mathrm{g}$ bid. Data were analysed by an all patients treated (APT) approach. Change from baseline in morning PEF was the primary variable. Secondary variables included asthma symptom score, $\beta_{2}$ agonist use, $\mathrm{FEV}_{1}$ and FVC. Treatments were compared by ANOVA. One hundred and seventy six patients were randomised and 171 (78/93 M/F; mean age 39 years) were valid for APT analysis. The mean morning PEF during run in was 408.9 (Bud TBH) and $408.5 \mathrm{l} / \mathrm{min}$ (FP DH). There were no clinically or statistically significant differences between the treatments in any of the variables recorded in diary cards or at clinic visits. Mean increase in morning PEF (adjusted for age and sex) was 8.0 (Bud TBH) and 16.6 (FP DH) $1 / \min (N S, p=0.35$ ). This study does not show any difference in efficacy between Bud Turbohaler and FP Diskhaler when halving the baseline dose of conventional steroids.

Ipratropium bromide given by Turbohaler ${ }^{\circledR}$ is more potent than when given by pressurised metered dose inhaler (MDI)

SP MATUSIEWICZ, FGE BÖLLERT, M DEWAR, G BROWN, A MCLEAN, AP GREENING, GK CROMPTON Respiratory Unit, Western General Hospital; Astra Clinical Research Unit, Edinburgh A study was undertaken to determine the relative potency of ipratropium bromide (IPBr) via Turbohaler in comparison with pressurised metered dose inhaler (MDI) in patients with reversible airflow obstruction. The design

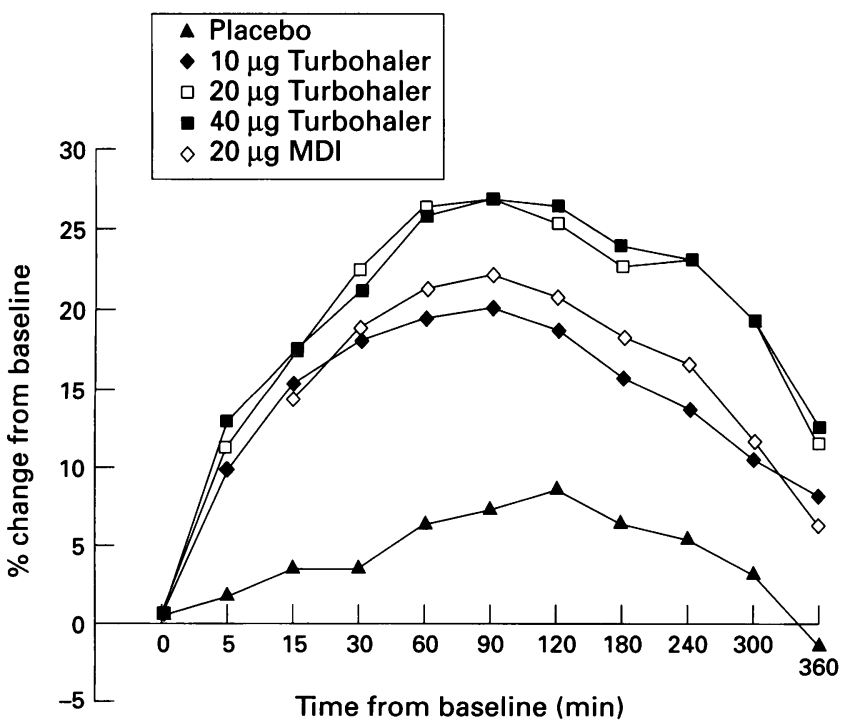

of the study was randomised, double blind, double dummy placebo controlled, single dose crossover study with one screening visit and five study visits. $\mathrm{FEV}_{1}$ was recorded five minutes before and 5, 15, $30,60,90,120,180,240,300,360$ minutes after drug administration. The end points of $\mathrm{FEV}_{1}$ and area under the curve (AUC) analysis were examined by analysis of variance. Thirty three patients $(21 \mathrm{men}$, mean age 55.8 years with reversible airflow obstruction $\left(\mathrm{FEV}_{1} 35-80 \%\right.$ of predicted and $\geqslant 1$ litre demonstrating $\geqslant 15 \%$ reversibility to $\mathrm{IPBr}$ )) completed the study. The \% change in $\mathrm{FEV}_{1}$ is shown in the figure. In clinically stable reversible airflow obstruction ipratropium bromide via Turbohaler elicited a dose dependent increase in $\mathrm{FEV}_{1}$ which peaked at $20-40 \mu \mathrm{g}$. AUC analysis showed that $20 \mu \mathrm{g}$ via Turbohaler was more effective than $20 \mu \mathrm{g}$ via MDI. The $20 \mu \mathrm{g}$ MDI was not different from $10 \mu \mathrm{g}$ Turbohaler. The efficacy ratio of Turbohaler: MDI was round $1.5 / 2.0$ and is similar to that demonstrated with other drugs administered by Turbohaler.

\section{Comparative efficacy of ipratropium bromide via Turbohaler ${ }^{\circledR}$} and MDI in patients with reversible airflow obstruction

FGE BÖLLERT, SP MATUSIEWICZ, M DEWAR, G BROWN, A MCLEAN, AP GREENING, GK CROMPTON Respiratory Unit, Western General Hospital; Astra Clinical Research Unit, Edinburgh Previous studies with other drugs have shown Turbohaler (TH) to be more effective than pressurised metered dose inhaler (MDI) (Thorax 1993;48:434; Chest 1994;105:697-700). We therefore aimed to compare the efficacy of ipratropium bromide (IB) via TH and MDI. A randomised, double blind, double dummy, crossover cumulative dose study was performed. A total dose of $160 \mu \mathrm{g}$ IB was given as individual doses of $20,20,40$ and $80 \mu \mathrm{g}$ via $\mathrm{TH}$ or MDI at 45 minute intervals on two study days at least 48 hours apart. $\mathrm{FEV}_{1}$ was measured prior to the first dose and 40 minutes after dosing. Analysis of variance for the end point $\mathrm{FEV}_{1}$ was performed. Fifteen patients (nine women, mean age 60 years, mean baseline $\mathrm{FEV}_{1} 1.521,57 \%$ of predicted normal with $\geqslant 15 \%$ reversibility to $40 \mu \mathrm{g}$ IB) completed the study. There was a steep dose-dependent increase in $\mathrm{FEV}_{1}$ irrespective of the inhaler device, $9 / 15$ patients on $\mathrm{TH}$ and 5/15 on MDI reached the maximum response at or before the cumulative dose of $40 \mu \mathrm{g}$ IB.

Mean (SD) cumulative dose response to IB via TH and MDI and

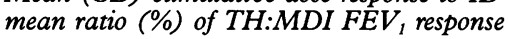

\begin{tabular}{|c|c|c|c|}
\hline \multirow{2}{*}{$\begin{array}{l}\text { Cum. } \\
\text { doses }\end{array}$} & \multicolumn{2}{|c|}{$F E V_{1}$ response } & \multirow{2}{*}{$\begin{array}{l}\text { Mean ratio (\%) adjusted } \\
\text { for period and baseline } \\
F E V_{1}(C I)\end{array}$} \\
\hline & $T H$ & $M D I$ & \\
\hline $\begin{array}{l}\text { Baseline } \\
20 \mu \mathrm{g} \text { IB } \\
40 \mu \mathrm{g} \text { IB }\end{array}$ & $\begin{array}{l}1.5(0.5) \\
1.79(0.6) \\
1.88(0.65)\end{array}$ & $\begin{array}{l}1.46(0.47) \\
1.71(0.54) \\
1.82(0.59)\end{array}$ & $\begin{array}{l}\text { N/A } \\
102 \cdot 9(98 \cdot 8-107 \cdot 1), p=0 \cdot 16 \\
100 \cdot 8(96 \cdot 1-105 \cdot 8), p=0.71\end{array}$ \\
\hline
\end{tabular}

There was no significant difference between the devices at any dose of IB. We conclude that there was no significant difference in the efficacy of TH and MDI at the dose range of IB tested, but further studies to assess the relationship at lower doses of IB might be warranted. A proportion of asthmatic patients respond to low doses of IB.

Comparison of the efficacy and tolerability of eformoterol and salbutamol dry powder capsules for inhalation in elderly asthmatic patients

RM ANGUS, NC THOMSON FOR A UK EFORMOTEROL STUDY GROUP Department of Respiratory Medicine, Western Infirmary, Glasgow Eformoterol is a selective $\beta_{2}$ agonist which when given by inhalation produces bronchodilation of rapid onset which lasts for 12 hours. The aim of this study was to examine the efficacy and tolerability of eformoterol dry powder in comparison with salbutamol dry powder in elderly asthmatic patients. A multicentre study, double blind, parallel group design was employed. Two hundred and sixty two patients (105 women) of mean (SD) age 71 (4) years, range 64-82 years, were studied. All had demonstrated at least $15 \%$ reversibility to inhaled salbutamol. Patients were randomised to receive either eformoterol $12 \mu \mathrm{g}$ bd, eformoterol $24 \mu \mathrm{g}$ bd, or salbutamol $400 \mu \mathrm{g}$ qid for a three month period. During a two week run in patients received salbutamol $400 \mu \mathrm{g}$ qid then entered one of the treatment arms. Sixty four patients were withdrawn during the study: 21 (25\%) 
eformoterol $12 \mu \mathrm{g}$ bd, 16 (18\%) eformoterol $24 \mu \mathrm{g} \mathrm{bd,} \mathrm{and} 27$ (31\%) salbutamol $400 \mu \mathrm{g}$ qid. Eformoterol $24 \mu \mathrm{g}$ and $12 \mu \mathrm{g}$ were statistically significantly different from salbutamol: the overall mean difference in the mean efficacy variable, pretreatment morning PEFR recorded daily by the patient, was estimated to be 33 and $34 \mathrm{l} / \mathrm{min}$ for eformoterol $12 \mu \mathrm{g}$ and $24 \mu \mathrm{g}$, respectively. Moreover, the mean pretreatment evening PEFR for eformoterol were both different from salbutamol by 32 and $30 \mathrm{l} / \mathrm{min}$ respectively. The daily use of rescue medication was less in the $24 \mu \mathrm{g}$ eformoterol group when compared with salbutamol. Analysis of asthma scores and sleep disturbance did not show a significant difference, however the patients' overall assessment of control was significantly better in both eformoterol groups when compared with salbutamol. Laboratory abnormalities did not seem to follow any particular pattern within the treatment groups. Similarly, there were no clinically significant changes in pulse, BP or ECG measurements. Overall tolerability was assessed by patients as good or very good for eformoterol $12 \mu \mathrm{g}(89 \%)$ and $24 \mu \mathrm{g}(88 \%)$ compared with (82\%) for salbutamol (NS). One patient in each of the $12 \mu \mathrm{g}$ eformoterol and salbutamol group had a serious adverse event that may have been drug related. These results suggest that eformoterol $12 \mu \mathrm{g}$ and $24 \mu \mathrm{g}$ bd provide superior asthma control and are tolerated at least as well as salbutamol $400 \mu \mathrm{g}$ qid in the elderly.

Regular formoterol treatment in mild asthma: effect on bronchial reactivity during and after treatment

DH YATES, $M$ WORSDELL, PJ BARNES, KF CHUNG Department of Thoracic Medicine, National Heart and Lung Institute and Royal Brompton Hospital, London There has been concern regarding the potential detrimental effects of $\beta_{2}$ adrenoceptor agonists on bronchial responsiveness and development of tachyphylaxis to their protective effects against bronchoconstriction in asthma. Formoterol, a new long acting $\beta_{2}$ agonist, is effective in single doses in the prevention of methacholine-induced bronchoconstriction. In a double blind, placebo controlled crossover study we examined the effect of regular formoterol treatment ( $24 \mu \mathrm{g}$ twice daily over two weeks) on airway calibre and methacholine $\mathrm{PC}_{20}$ in 17 mild steroid naive asthmatics. $\mathrm{FEV}_{1}$ and bronchial reactivity to methacholine were also measured after treatment cessation at $12,36,60$ and 108 hours and at two weeks in order to examine for a "rebound" increase in BHR. FEV and $\mathrm{PC}_{20}$ rose significantly with formoterol $(3.441$ to 3.791 ; and $0.53 \mathrm{mg} / \mathrm{ml}$ to $2 \mathrm{mg} / \mathrm{ml} ; \mathrm{p}<0.005$ compared with placebo) measured 12 hours after one dose. This effect was not maintained after two weeks treatment, $\mathrm{FEV}_{1}$ falling to 3.511 and $\mathrm{PC}_{20}$ to $0.93 \mathrm{mg} / \mathrm{ml}$ $(p<0.007$ and $p<0.04$ respectively compared with placebo), but both remained significantly higher than baseline. No significant rebound decrease in $\mathrm{PC}_{20}$ nor in $\mathrm{FEV}_{1}$ was manifest compared with placebo. Significant tachyphylaxis to both the bronchodilator and bronchoprotective effects of formoterol thus occurred. There was no evidence of rebound BHR. Regular usage of formoterol may therefore be accompanied by partial loss of its beneficial effects. The clinical significance of these findings remains to be determined. [We thank Ciga-Geigy, Switzerland for supply of formoterol and for financial support.]

Efficacy of once daily budesonide via Turbohaler ${ }^{\circledR}$ in moderate asthma

LM CAMPBELL ON BEHALF OF THE PATRON INVESTIGATORS GROUP Southbank Surgery, Kirkintilloch, Glasgow Once daily budesonide has been shown to be as effective as twice daily in mild asthma (Jones et al. Respir Med 1994;88:293-6). We undertook this study to test the concept of once daily inhaled budesonide in the control of more severe asthma. Patients who were receiving $400-600 \mu \mathrm{g}$ inhaled steroid daily entered a one week run in to assess peak expiratory flow rate (PEFR), asthma symptoms, and rescue $\beta_{2}$ agonist use. One hundred and sixty four symptomatic patients were randomised to open, parallel group treatment with $800 \mu \mathrm{g}$ budesonide via Pulmicort $\AA$ Turbohaler $\AA$ administered either as $800 \mu \mathrm{g}$ once daily (nocte) or as $400 \mu \mathrm{g}$ twice daily. PEFR and symptoms were reviewed at clinic visits at four and eight weeks, and patients kept a daily record of symptoms, PEF (am and pm) and $\beta_{2}$ agonist use. Morning PEF recorded in diary cards increased by $211 / \mathrm{min}$ in the twice daily group $(p<0.0001)$ and by $29 \mathrm{l} / \mathrm{min}$ in the once daily group by eight weeks $(p<0.001)$, with overall improvement in symptom scores in both groups. Diary card records also showed significant improvements in
PEFR and reduction in diurnal variation, with reduced $\beta_{2}$ agonist use. Day time and night time symptoms were reduced in both groups. There were no significant differences found between the treatment groups for any of the variables tested. We conclude that $800 \mu \mathrm{g}$ budesonide can be given equally effectively either as a once daily or twice daily dose via Turbohaler.

RFLP analysis of the P2 gene of Haemophilus influenzae isolates recovered from patients with bronchiectasis

A PYE, RA STOCKLEY, TF MURPHY, SL HILL Lung Immunobiochemical Research Laboratory, The General Hospital, Birmingham; Suny at Buffalo, Buffalo, USA P2 is the major outer membrane protein of nontypeable Haemophilus influenzae (NTHI) and has been shown to express a highly strain specific immunodominant epitope. Phenotypic changes have been demonstrated in this protein in isolates recovered longitudinally from patients with bronchiectasis. Such changes in P2 could provide a mechanism for NTHI to evade lung host defence systems thereby allowing its persistence in the lower respiratory tract. We report the preliminary results of RFLP analysis of the P2 gene in 52 isolates of NTHI collected from 10 patients with bronchiectasis. RFLP analysis was performed on a PCR amplified gene segment (using appropriate oligonucleotide strain) and the restriction endonuclease $\mathrm{Xbal}$ (which has three recognition sites in this segment of the reference strain). Nine of the 52 isolates (eight from one patient) had no Xbal sites anywhere in the amplified segment. The restriction patterns also revealed that none of the isolates had the Xbal restriction site in the variable region of loop 4 . In the remaining isolates a variety of restriction patterns were observed for either or both of the two other restriction sites in conserved regions. In the 10 individual patients, RFLP analysis showed that three of four patients' isolates collected sequentially showed different P2 restriction patterns even though P2 was phenotypically similar on SDS-PAGE. In five of the remaining six patients where the phenotype of P2 differed on SDS-PAGE in isolates collected sequentially there were corresponding differences in RFLP. In conclusion, changes in the P2 gene occur more frequently in NTHI isolates from patients with bronchiectasis than have previously been suggested, even in the presence of apparently similar phenotype on SDS. Further studies are required to relate these changes to the expression of relevant antigenic epitopes.

An air interface organ culture model for use in the study of the interactions of bacteria, viruses and pharmacological agents with the human respiratory mucosa

AD JaCkson, CFJ RAYNer, A DEWAR, PJ COLE, R wilson Host Defence Unit, Department of Thoracic Medicine, Royal Brompton National Heart and Lung Institute, London The mucociliary epithelium lining the respiratory tract is the primary interface between airborne particles, vapours and gases, and the host epithelium. Respiratory organ cultures that are immersed in culture medium are unphysiological. We have assessed a human respiratory tissue organ culture model with an air interface by light microscopy, scanning electron microscopy (SEM), and transmission electron microscopy (TEM). Dissected nasal turbinate tissue was soaked in antibiotic medium to remove commensal flora, and was maintained in a humidified atmosphere with $5 \% \mathrm{CO}_{2}$ on a filter paper strip whose ends were immersed in cell culture medium. Without changing the culture medium ciliary beat frequency (CBF) remained constant $(11-12 \mathrm{~Hz})$ over five days but fell significantly $(p=0.004)$ at 10 days to $7.9(0.8) \mathrm{Hz}$. By SEM, percentage tissue cover by mucus varied from $29-84 \%$ with no significant time related trend. The percent of the surface covered by ciliated cells was significantly reduced at five and 10 days. By TEM nuclear heterochromatin was reduced at five days and 10 days. Mitochondria had normal ultrastructure at four days but appeared slightly abnormal at five days and severely damaged at 10 days. Cilial density on individual ciliated cells was significantly reduced at 10 days. Tight junctions between cells, cell projection from the organ culture surface, cytoplasmic blebbing and cell vacuolation remained normal over 10 days. When the culture medium was replaced daily TEM indicated that mitochondria and nuclei were normal, CBF was between 10 and $11 \mathrm{~Hz}$, and numbers of ciliated cells and cilial density on individual cells were normal after 20 days. This model is simple to construct and reproduces physiological conditions in vitro. Because of its long viability it can be used to study virus and bacterial infections, and the effects of pharmacological agents and environmental factors. 
Recognition of outer membrane proteins of non-typeable Haemophilus influenzae by IgG subclass antibodies in serum and sputum from patients with bronchiectasis

JL MITCHELL, SL HILL Lung Immunobiochemical Research Laboratory, The General Hospital, Steelhouse Lane, Birmingham Non-typeable Haemophilus influenzae (NTHI) is frequently isolated from the sputum of patients with bronchiectasis. The organism persists in the airways of these patients despite the presence of an exuberant inflammatory response which includes a significant local production of immunoglobulin in the lung secretions. We have therefore compared the NTHI-specific IgG subclass responses in pooled sputum and serum collected from six patients with bronchiectasis to assess the relative importance of outer membrane proteins (OMP) or NTHI in eliciting antibody responses in the lung and blood. OMP complexes from eleven subtypes (based on the relative molecular weight of the proteins P2 and P5 on SDS-PAGE) of NTHI were subjected to SDSPAGE, blotted onto nitrocellulose and then incubated in the presence of either the serum pool, or the sol phase sputum pool. This was followed by incubation with biotinylated polyclonal antibodies to one of the four human IgG subclasses and colour development with HRPconjugated avidin-biotin complexes. In addition, the outer membrane protein P6 was purified from NTHI and used alone in similar blotting experiments. The results showed recognition of all of the major OMPs of the subtypes of NTHI tested. Some antigens were more strongly recognised than others by one or more subclasses of IgG in the serum and sputum pools. Of major importance was the strong recognition (particularly in sputum) of a $16 \mathrm{kD}$ antigen consistent with the OMP P6 detected by immunoblot with sputum at titres as low as $1 / 1250$. There was no recognition of the band in the $16 \mathrm{kD}$ position after the whole cell lysate of NTHI was subjected to proteinase $\mathrm{K}$ digestion confirming that this result was not due to contaminating lipooligosaccharide. In addition similar results were obtained with the purified P6. These experiments suggest that P6 is an importan immunogen in the airways of patients with bronchiectasis. Further studies are necessary to establish the functional role of P6 specific antibodies in the lung secretions of these patients.

Effect of erythromycin on Haemophilus influenzae endotoxin (HIE)-induced release of inflammatory mediators by human bronchial epithelial cells (HBEC) in vitro

OA KHAIR, JL DEVALIA, RJ SAPSFORD, MM ABDELAZIZ, RJ DAVIES Department of Respiratory Medicine and Allergy, St Bartholomew's Hospital, London Although several studies have demonstrated that low dose, long term erythromycin (ERM) treatment is effective in the management of chronic lower respiratory tract infections such as chronic bronchitis and bronchiectasis, the mechanisms underlying the action of ERM are not clear. We have cultured HBEC as explant cultures from surgical tissue and investigated the effect of ERM on HIE-induced release of inflammatory mediators in these cultures. Confluent cultures were incubated for 24 hours with $100 \mu \mathrm{g} / \mathrm{ml} \mathrm{HIE}$ and analysed for interleukins 6 and 8 (IL-6 and IL-8) and soluble intercellular adhesion molecule 1 (sICAM-1) released into the culture medium. Additionally, cultures were incubated in the presence of $0 \cdot 1-10 \mu \mathrm{g} / \mathrm{ml}$ ERM and investigated for the effect of this antibiotic on HIE-induced release of IL-6 and IL-8. IL-6, IL-8 and SICAM-1 in the medium were analysed by ELISA. HIE significantly increased the release of IL- 6 and IL-8, from $3.9(1.5) \mathrm{pg}$ IL-6/ $\mu \mathrm{g}$ cellular protein

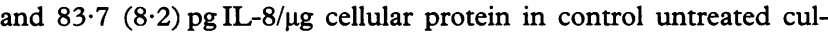
tures, to $12 \cdot 12(1 \cdot 5) \mathrm{pg} \mathrm{IL}-6 / \mu \mathrm{g}$ cellular protein $(\mathrm{p}<0.005)$ and $225 \cdot 7$ $(44.8) \mathrm{pg} \mathrm{IL}-8 / \mu \mathrm{g}$ cellular protein $(\mathrm{p}<0.05)$, respectively. Similarly, HIE significantly increased the release of sICAM-1 from 0.23 $(0.03) \mathrm{ng}$ sICAM-1/ $\mu \mathrm{g}$ cellular protein in control cultures, to 3.82 $(0.9) \mathrm{ng}$ sICAM-1/ $\mu \mathrm{g}$ cellular protein $(\mathrm{p}<0.001)$. Incubation of HBEC in the presence of $0 \cdot 1-10 \mu \mathrm{g} / \mathrm{ml}$ erythromycin significantly blocked the HIE-induced release of both IL-6 and IL-8. These results suggest that $H$ influenzae-induced release of inflammatory mediators from airway epithelial cells may contibute to chronic airway inflammation and that this effect may be modulated by erythromycin treatment.

Late as well as early neutrophil influx is CD18 dependent in $E$ coli pneumonia

RA LAWSON, C HASLETT Rayne Laboratory, Department of Respiratory Medicine, RIE, Edinburgh A prerequisite for resolving inflammation is cessation of neutrophil influx. A mechanism by which this might be controlled is switching of adhesion molecule dependence. Winn and Harlan ( $\mathcal{F}$ Clin Invest 1993;92:1168-73) showed that late neutrophil influx into the peritoneum becomes CD18 independent. The requirement for CD18 differs in pulmonary and systemic circulations. Hence we studied CD18 requirement early and later in pneumonia. Pairs of rabbits had $E$ coli instilled bronchoscopically into the right cranial lobe. After six or 30 hours they received either anti-CD18 (Celltech) or saline followed by ${ }^{111}$ In labelled neutrophils from a donor. Six and 12 hours later they received repeat antibody or saline and after 20 hours received ${ }^{99 \mathrm{~m}}$ Tc labelled red blood cells. A dual isotope gamma camera image was obtained. One hour after this the lungs were lavaged. Lavagate and tissue gamma activity were counted. The neutrophils accumulating from six or 30 hours were calculated as the indium accumulating in excess of that in the blood pool as a

\begin{tabular}{|c|c|c|c|c|c|}
\hline & \multirow{2}{*}{$\begin{array}{l}0 \text { hours } \\
\text { No } \\
\text { treatment }\end{array}$} & \multicolumn{2}{|c|}{6 hours } & \multicolumn{2}{|c|}{30 hours } \\
\hline & & $\mathrm{NaCl}$ & $\begin{array}{l}\text { Anti- } \\
C D 18\end{array}$ & $\mathrm{NaCl}$ & $\begin{array}{l}\text { Anti- } \\
C D 18\end{array}$ \\
\hline $\begin{array}{l}\text { Lavage } \\
\text { Tissue } \\
\text { Scan }\end{array}$ & $\begin{array}{c}0.002 \\
0.33 \\
-1.40\end{array}$ & $\begin{array}{l}0.062 \\
5 \cdot 30 \\
5 \cdot 76\end{array}$ & $\begin{array}{l}0.042 \\
1.85^{*} \\
1.52^{*}\end{array}$ & $\begin{array}{l}0.011 \\
3.44 \\
1.65\end{array}$ & $\begin{array}{l}0.007 \\
1 \cdot 12^{*} \\
0 \cdot 80^{*}\end{array}$ \\
\hline
\end{tabular}

* Significant differences between pairs (paired $t$ test, $\mathrm{p}<0.05$ ).

Results are means of $n=7$. Controls are untreated $(n=17)$.

percentage of injectate. The results show that neutrophil influx was significantly (though not completely) inhibited by anti-CD18 antibody, not only at early (six hour) but also at late (30 hour) time points. Lavage neutrophil accumulation was not significantly affected, perhaps because of a greater effect on sequestration than migration, or because anti-CD18 coated cells are easier to lavage out of the lungs. [Work supported by MRC (UK). Anti-CD18 supplied by Celltech.]

Analysis of lymphocytic proliferation and interleukin production in response to CMV antigen following lung transplantation

M HEALY, S HAMILTON, $\mathrm{R}$ SCOTT, $\mathrm{K}$ GOULD, JH DARK, PA CORRIS Cardiopulmonary Transplant Unit, Department of Microbiology, Freeman Hospital, Newcastle upon Tyne; Department of Virology, Newcastle University As part of a prospective study conducted over the last two years blood and BAL lymphocyte cultures were tested for their degree of CMV specific lymphocyte proliferation using a triated thymidine uptake assay. This was done at the time of routine (one week, one month, three month, six month, and yearly) and emergency diagnostic bronchoscopies. Culture supernatants were tested for IL2 and IL-4 levels using Medgenix ELISA kits. There was no association between LPB (lymphocyte proliferation blood) or LPL (lymphocyte proliferation lavage) positivity and CMV excretion/non-excretion or CMV antigenaemia at the time of the bronchoscopy. There was a significantly higher rate of LPB positivity $(p=0.0015)$ amongst the group with a history of CMV excretion at some time than those who never excreted CMV. This suggests that the LPB positivity may predate or postdate other episodes of excretion that our surveillance and diagnostic bronchoscopies did not detect. There is no association at the time of bronchoscopy between LPB or LPL and clinical status, rejection incidence, infection incidence, or OB. Previous work presented at the BTS has shown that asymptomatic CMV excretion from the lungs is not associated with a greater incidence of infection, rejection, or problems with lung function. The finding of IL-2 or IL4 in the supernatant following LPB positivity is almost significantly greater amongst the group with a history of CMV excretion (analysis not limited to episodes of CMV excretion) compared with the group who never excreted CMV. The finding of IL-2 or IL-4 in the supernatant following LPB or LPL positivity does not correlate, at the time of bronchoscopy, with infection, rejection, or clinical status. The three patients in the study who had CMV disease (two died, one survivor) never had a LPB or LPL response. Perhaps the exuberant/ disordered immune response in CMV disease reflects an inability of the patients' lymphocytes to respond to CMV antigen. Patients who had histological evidence of CMV cells (cytopathological effects) but no other inflammatory changes exhibited similar LPB and LPL responses to the CMV excretor group. They did not mimic the CMV disease group in consistently negative LPB and LPL. [This study was supported by British Lung Foundation.] 
Effect of salmeterol on the interaction between Pseudomonas aeruginosa (PA) and the respiratory mucosa in vitro

R DOWLING, C RAYNER, A JACKSON, G TAYLOR, M JOHNSON, PJ COLE, R wILson Host Defence Unit, Department of Thoracic Medicine, Royal Brompton National Heart and Lung Institute, London Salmeterol is a potent long acting $\beta_{2}$ agonist which we have previously shown to reduce the ciliary beat slowing and epithelial disruption caused by the Pseudomonas aeruginosa (PA) toxin pyocyanin ( $\mathrm{Br} \mathcal{F}$ Pharmacol 1994;112:493-8). We have studied the effect of salmeterol on PA interaction with the respiratory mucosa of an organ culture with an air-mucosal interface by scanning electron microscopy. Human adenoid or turbinate tissue was incubated with $4 \times 10^{-7} \mathrm{M}$ salmeterol for 30 minutes prior to inoculation with PA (A), or $20 \mu \mathrm{l} 4 \times 10^{-7} \mathrm{M}$ salmeterol was pipetted onto the organ culture surface immediately prior to bacterial inoculation (B). Infected organ cultures at eight hours showed a significant $(p \leqslant 0.02)$ increase in epithelial damage compared with control, and PA was predominantly associated with mucus and damaged cells. Salmeterol significantly $(A: p \leqslant 0 \cdot 04, B$ : $p \leqslant 0.05)$ reduced epithelial damage caused by PA infection. Salmeterol had no effect on either the number or distribution of PA

\begin{tabular}{|c|c|c|c|c|}
\hline & \multicolumn{4}{|c|}{ Percentage of organ culture surface } \\
\hline & Mucus & Damaged & Ciliated & Unciliated \\
\hline \multirow{3}{*}{$\begin{array}{l}\text { A: control } \\
\text { PA } \\
\text { PA and } \\
\text { salmeterol } \\
\text { B: control } \\
\text { PA } \\
\text { PA and } \\
\text { salmeterol }\end{array}$} & $\begin{array}{l}2 \cdot 9(0 \cdot 7) \\
4 \cdot 7(0 \cdot 8)\end{array}$ & $\begin{array}{l}13.7(4.2) \\
45.6(8.4)\end{array}$ & $\begin{array}{l}36.5(11.5) \\
14.2(9.5)\end{array}$ & $\begin{array}{l}46.9(9.3) \\
35.5(10.0)\end{array}$ \\
\hline & $\begin{array}{r}11.5(9.5) \\
3.9(0.9) \\
8.0(4.0)\end{array}$ & $\begin{array}{l}24 \cdot 0(11 \cdot 0) \\
15 \cdot 0(5 \cdot 2) \\
53.4(9 \cdot 7)\end{array}$ & $\begin{array}{l}35.0(11 \cdot 1) \\
32.5(7 \cdot 0) \\
20.2(9.4)\end{array}$ & $\begin{array}{l}29 \cdot 5(9.8) \\
48.6(4.9) \\
18 \cdot 4(4.6)\end{array}$ \\
\hline & $6 \cdot 8(3 \cdot 2)$ & $24 \cdot 0(8 \cdot 3)$ & $41.6(11.7)$ & $27 \cdot 6(7 \cdot 1)$ \\
\hline
\end{tabular}

adhering to the mucosa. These results show that salmeterol reduces the damage caused by PA infection of the respiratory mucosa in vitro and may benefit patients infected by PA by this mechanism as well as bronchodilatation.

\section{Inhibition of substance $P$ degradation and its effect on human respiratory ciliary activity in vitro}

RP SMITH, $\mathrm{R}$ SHELLARD, G DI BENEDETTO, A MEHTA Department of Respiratory Medicine, King's Cross Hospital, Dundee; Department of Child Health, Ninewells Hospital and Medical School, Dundee; Department of Thoracic Medicine, Royal Free Hospital, London The tachykinin substance $P(S P)$ is an important mediator in many inflammatory conditions of the lung. We have previously demonstrated that SP $\left(10^{-8}-10^{-6} \mathrm{M}\right)$ produces an immediate rise in intracellular calcium which is associated with a transient increase in ciliary beat frequency (CBF) (Eur Respir F 1991;4(Suppl 14):291S). We hypothesised that the breakdown of SP (mediated by the membrane-bound enzyme neutral endopeptidase) was responsible for the rapid termination of this $\mathrm{CBF}$ response. Here we studied the $\mathrm{CBF}$ response to $\mathrm{SP}\left(10^{-6} \mathrm{M}\right)$ in the presence of the endopeptidase inhibitor phosphoramidon $\left(10^{-6} \mathrm{M}\right)$. Respiratory epithelial cells, obtained by brushing the inferior turbinates of 10 normal volunteers, were suspended in medium 199 and placed in a perfusion chamber. CBF was measured using a videomicroscopy system (Respir Med 1994;88:89-101) and the rise in CBF to one of the following was observed: (1) phosphoramidon alone, (2) substance $P$ alone (3) phosphoramidon + substance $P$. We found that phosphoramidon alone had no effect on baseline CBF and confirmed that SP alone produced a transient rise. However, when SP and phosphoramidon were perfused together the transient response was no longer present but was replaced by a delayed rise which was significantly elevated above control at 25 minutes (table). The time

Mean (SE) \% change in CBF (n=10)

\begin{tabular}{llllll}
\hline Perfusate & $10 \mathrm{~min}$ & $15 \mathrm{~min}$ & $20 \mathrm{~min}$ & $25 \mathrm{~min}$ & $30 \mathrm{~min}$ \\
\hline SP alone & 109 & 106 & 104 & $103^{*}$ & 101 \\
& $(4 \cdot 6)$ & $(2 \cdot 8)$ & $(3 \cdot 0)$ & $(2 \cdot 6)$ & $(2 \cdot 3)$ \\
SP + phosphoramidon & 100 & 108 & 111 & $112^{*}$ & 110 \\
& $(2 \cdot 3)$ & $(3 \cdot 9)$ & $(4 \cdot 5)$ & $(5 \cdot 0)$ & $(5 \cdot 6)$ \\
\hline
\end{tabular}

${ }^{*} \mathrm{p}<0 \cdot 01$, unpaired $t$ test. course of this delayed rise in CBF is similar to that we have previously reported with calcium ionophore A23187 ( $F$ Physiol 1991;439:10313). Furthermore, the delayed response was abolished by the calcium channel blocker lanthanum chloride $(250 \mu \mathrm{M})$. We conclude that inhibition of neutral endopeptidase activity modifies the time course of the SP-induced increase in CBF to produce a calcium dependent, delayed rise in $\mathrm{CBF}$.

\section{Primary and secondary ciliary disorientation}

A RUTMAN, CFJ RAYNER, A DEWAR, MA GREENSTONE, PJ COLE, R WILson Host Defence Unit, Royal Brompton National Heart and Lung Institute, London Cilia on the same cell normally beat in the same direction, which can be assessed by electron microscopy. Patients with ciliary disorientation (CD) have impaired mucociliary clearance (MCC) because cilia on the same cell beat in different directions. $\mathrm{CD}$ can be measured by the standard deviation (SD) of the angles that the ciliary axes on a cell make with the perpendicular - that is, the higher the SD, the greater the CD. CD may occur secondary to viral infection or chronic inflammation due to infection, but two case reports ( $N$ Engl f Med 1992;323:1681-4; Thorax 1993;48:70-1) have suggested that $C D$ alone (patients have absent MCC but motile cilia and normal ciliary ultrastructure) can be inherited and cause Kartagener's syndrome (KS; primary ciliary dyskinesia). We have investigated a family in which two siblings have KS but motile cilia with normal ultrastructure. We have also treated (antibiotics and topical corticosteroids) one patient with chronic mucopurulent sinusitis and two patients with $\mathrm{KS}$ and $\mathrm{CD}$ alone to determine if $\mathrm{CD}$ is reversible. The mother, father and a healthy sibling did not have CD (SD of angles $<15 \cdot 6^{\circ}$ ), whereas the two affected siblings had CD which was unchanged in biopsies taken 10 years apart $\left(22.6^{\circ}\right.$ and $26 \cdot 7^{\circ} ; 26 \cdot 4^{\circ}$ and $25 \cdot 0^{\circ}$ ). Right and left bronchial cilia from one sibling were also disorientated $\left(23 \cdot 5^{\circ}, 22 \cdot 2^{\circ}\right)$. In sinusitis CD was reversible $\left(21 \cdot 5^{\circ}-10 \cdot 3^{\circ}\right)$ but in two $\mathrm{KS}$ patients with $\mathrm{CD}$ alone it was not $\left(25 \cdot 2^{\circ}-24 \cdot 5^{\circ} ; 23 \cdot 8^{\circ}-23 \cdot 1^{\circ}\right)$. The presence of CD in two siblings, in biopsies taken 10 years apart, in cilia from different parts of the respiratory tract, and the lack of reversibility in such patients compared with mucopurulent sinusitis, supports the hypothesis that $C D$ alone (primary CD) can cause $\mathrm{KS}$.

Ciliary disorientation alone can cause primary ciliary dyskinesia syndrome

CFJ RAYNER, A RUTMAN, A DEWAR, MA GREENSTONE, PJ COLE, R wilson Host Defence Unit, Department of Thoracic Medicine, Royal Brompton National Heart and Lung Insitute, London; Hull Royal Infirmary, Hull Two case reports have proposed that ciliary disorientation alone may cause the syndrome of primary ciliary dyskinesia (PCD). These cases had motile cilia and normal ciliary ultrastructure but inefficient transport because the direction of the ciliary beat was disorientated. We have identified 11 patients with the clinical features of PCD who have normal ciliary ultrastructure. A clinical assessment, chest radiograph, pulmonary function tests, nasal mucociliary clearance (NMCC), ciliary beat frequency (CBF), ciliary ultrastructure and orientation were assessed in each subject and eight of the $11 \mathrm{had}$ computerised tomography of the thorax. The clinical features were the same as those previously reported in patients with PCD: chronic cough and sputum production, bronchiectasis, chronic sinusitis, middle ear problems, dextrocardia (in five) and infertility (one male had immotile sperm). Cilia ultrastructure was normal but NMCC was absent in all cases. The CBF ranged from $8 \cdot 4-14 \cdot 9 \mathrm{~Hz}$. Ciliary beat pattern was stiff in seven cases, six of whom had slow $(<11 \mathrm{~Hz})$ CBF. Ciliary disorientation was measured using an Improvision image analysis system. The cilia were significantly disorientated in all cases when measured by both the central pair (range $21 \cdot 8-26.4^{\circ}$ ) and basal feet (range $20 \cdot 6-28 \cdot 9^{\circ}$ ) compared with 16 normal controls (range $11 \cdot 0-15 \cdot 5$ and $12 \cdot 3-17 \cdot 6$, respectively). This study suggests that ciliary disorientation alone can lead to the clinical syndrome of PCD. Ciliary disorientation should be assessed if NMCC is absent, CBF is normal or near normal, and ciliary ultrastructure is normal.

Ciliary function tests: can we detect recurrent microaspiration in children with gastro-oesophageal reflux?

WA MCCALlion, WAN PO A LI, SR POTTS Royal Belfast Hospital For Sick Children, Belfast Respiratory tract disease associated with gastro- 
oesophageal reflux (GOR) may be reflex vagally mediated or result from aspiration. Our aim was to determine if ciliary motility is impaired by in vitro aspiration of gastric juice (GJ) or oropharyngeal contents and hence to explore the potential of ciliary function tests in the diagnosis of recurrent microaspiration in children with GOR. Cilia from the inferior turbinates of healthy volunteers were obtained by brush cytology. Control ciliated epithelium was incubated in growth medium at $37^{\circ} \mathrm{C}$. The remaining ciliated cells were exposed transiently (in vitro aspiration) at $37^{\circ} \mathrm{C}$ to milk, saliva and four solutions of GJ in which the concentration of $\mathrm{HCl}$ and pepsin varied, followed by "rescue" into growth medium. Ciliary beat frequency (CBF) was measured using a computerised photometric technique. The odds of ciliated cellular death was calculated. Interaction between GJ components was assessed by factorial design analysis. In vitro aspiration of milk and saliva had no effect on ciliary function whereas transient exposure to GJ impaired CBF $(p<0.05)$ and increased the odds of ciliated cellular death by $2 \cdot 5-8 \cdot 5$ times ( $95 \%$ confidence interval). Increasing the $\mathrm{HCl}$ or pepsin concentration further enhanced the ciliotoxic effect of GJ but there is no synergism. Transient exposure of ciliated epithelium to GJ significantly impairs ciliary motility. An assessment of tracheal ciliary function may be useful in distinguishing recurrent microaspiration of GJ in children with GOR from aspiration direct from the oropharynx or vagally mediated respiratory symptoms.

\section{Breaking the "bad news" of lung cancer: a study of patients' views and support needs}

M CAMpbell, a hughes, J Macfarlane Respiratory Medicine, City Hospital, Nottingham We studied 20 consecutive patients who had been told their diagnosis of lung cancer in our combined lung oncology clinic (CLOC) within the previous two weeks and had seen our Macmillan support sister (MC) at the same time. Our normal practice is to review the patients again in the clinic 2-3 weeks later and to telephone the GP from the clinic when bad news is broken. All patients were contacted by phone by MC within two weeks after the diagnosis discussion and asked questions in a semi-structured manner. Ten patients had "felt worse" since knowing the diagnosis, in only two cases due to deteriorating physical symptoms. Ten felt unchanged or better. Eleven patients had had no professional contact following the clinic visit, six had been contacted by their GP, and three by other staff. Eighteen felt they had understood all or most of the information given in the CLOC but 15 would have liked further discussions shortly after about the diagnosis and their concerns. Of those five patients who did not wish further discussions, three had families who did want more information. Concerns raised mainly involved fear of the treatment and investigations (eight) and uncertainty about their future (six); only two did not understand the information provided initially. Fourteen patients said they would have benefited from a home visit mostly within the first week, two would have liked a visit in the future, two were happy with the planned clinic support, and two were quite content with the support from the GP/other staff. We conclude that, although most patients understand the information given to them at the time of diagnosis, there is a big need for rapid follow up and contact from the lung cancer team, possibly in the form of a home visit by a Macmillan support sister.

\section{Lung cancer in ex-smokers}

M MUNAVVAR, BR O'DRISCOLl Salford Royal Hospitals NHS Trust, Salford It is frequently stated that ex-smokers have a negligible risk of developing lung cancer once they have stopped smoking for more than 10 years (BMf 1994;308:1479). However, Doll and Hill reported that doctors who gave up smoking even 20 years previously had a threefold greater incidence of fatal lung cancer than life long nonsmokers $(0.19 v 0.07$ deaths per 1000) (BMf 1964;1:1399-410). We have recorded the smoking status prospectively in 872 patients referred

\begin{tabular}{lcc}
\hline & $\begin{array}{l}\text { Doll and Hill } \\
(144 \text { tumours })\end{array}$ & $\begin{array}{l}\text { Salford } \\
(n=274)\end{array}$ \\
\hline Current smokers & $86 \%$ & $55 \%$ \\
Ex smokers & & \\
$0-10$ years & $8 \%$ & $26 \%$ \\
$10-20$ years & $2 \%$ & $8 \%$ \\
$>20$ years & $1 \%$ & $4 \%$ \\
Non smokers & $2 \%$ & $1.5 \%$ \\
Other (pipe, mixed etc) & & $5 \%$ \\
\hline
\end{tabular}

for fibreoptic bronchoscopy. Of these $274(31 \%)$ were found to have a visible tumour in the central airways. The smoking status of these patients is shown in the table and the data of Doll and Hill are shown for comparison. We conclude that longstanding ex-smokers have a reduced (but not negligible) risk of lung cancer.

\section{Risk of lung cancer following radiotherapy for breast cancer} in Yorkshire

HSR HOSKER, O JOHNSON, T PAYNE, C JOHNSTON, SB PEARSON LeedS Chest Clinic and Cancer Registry, Cookridge Hospital, Leeds Recent studies suggest that radiotherapy for breast cancer increases the risk of lung cancer, but there are no available data from the UK. A retrospective case note study was set up to examine any excess risk in patients with breast cancer treated with radiotherapy in Yorkshire. All patients with proven breast cancer between 1976 and 1993 and a subsequent diagnosis of lung cancer at least six months later were identified. Patients with inadequate data, or lung histology suggesting adenocarcinoma, were excluded. Patients were compared with 660 matched case controls with breast cancer diagnosed in the same year who had not developed lung cancer. The number of patients from each group who had received radiotherapy for breast cancer was compared. Ninety nine cases were identified with breast cancer and subsequent lung cancer. Thirty three of these had proven lung cancer histology which was not adenocarcinoma, 21 (64\%) of whom had received radiotherapy for breast cancer, compared with 288 of 660 $(44 \%)$ of the case controls who had not developed lung cancer. The relative risk of prior radiotherapy was $2 \cdot 3(95 \% \mathrm{CI} 1 \cdot 1$ to $4 \cdot 9 ; \mathrm{p}=0.03)$. Histological types of lung cancer were similar in the radiotherapy and non-radiotherapy groups, with $61 \%$ being squamous, $27 \%$ small cell, and $9 \%$ poorly differentiated. There was one case of non-Hodgkin's lymphoma. These results suggest that patients with breast cancer who subsequently develop lung cancer are twice as likely to have received radiotherapy for breast cancer than patients who do not develop lung cancer, supporting the hypothesis that such radiotherapy increases the risk of subsequent lung cancer.

\section{Completion pneumonectomy for lung cancer: indications and} outcome

K AL Kattan, P Goldstraw Royal Brompton Hospital, London Completion pneumonectomy for lung cancer has been associated with higher rates of mortality and morbidity and this is reflected in the selection of cases. Over a period of 14 years (January 1980 to December 1993) 24 completion pneumonectomies for lung cancer were performed representing $4.5 \%$ of all pneumonectomies for lung cancer. There were 15 right and nine left completion pneumonectomies (CP) for an average age of 64 years (range 39-77). The indication for CP included local recurrence in 10 , a second in nine, and a new primary after previous pulmonary resection for tuberculosis in two patients. Operative complications requiring $\mathrm{CP}$ after resection for lung cancer included two bronchial stenosis and one empyema. There was one operative death and $6.7 \%$ morbidity in form of bleeding in two, prolonged ventilation in one and empyema in one. We conclude that $C P$ can be performed with an acceptable risk in selected patients. This aggressive approach should only be performed as a potentially curative procedure.

Value of cervical mediastinoscopy combined with anterior mediastinotomy in bronchogenic carcinoma of the left upper lobe

x JiAO, P Goldstraw Royal Brompton Hospital, London In the preoperative evaluation of a patient with lung cancer assessment of the mediastinum to exclude invasion and proximal nodal disease remains a fundamental importance. In this respect, cervical mediastinoscopy is of proven benefit but the development of scanning techniques have allowed this invasive procedure to be applied selectively. The situation is complicated for patients with tumours within the left upper lobe since cervical mediastinoscopy cannot exclude nodal disease beneath the aortic arch, lateral to the aortic arch or within the anterior mediastinum, nor exclude direct tumour invasion in this area. The addition of left anterior mediastinotomy allows biopsy of the subaortic lymph nodes, bidigital palpation of the subaortic fossa and, if necessary, the intrapericardial exploration. We have sought to evaluate 
the contribution that this additional minor operation makes to the preoperative selection of patients with tumours within the left upper lobe. From January 1990 to July 1994, 85 patients who were otherwise thought to have an operable tumour within the left upper lobe underwent cervical mediastinoscopy and left anterior mediastinotomy. Twenty seven $(31.8 \%)$ patients were found to be inoperable, either because of nodal involvement at cervical mediastinoscopy (four patients) or because of extension into the mediastinum at left anterior mediastinotomy (14 patients), or because of positive results from both methods (nine patients). The inoperability determined by this examination for patients with adenocarcinoma $(8 / 18,44.4 \%)$ is higher than for patients with squamous carcinoma $(12 / 52,23 \cdot 1 \%)$. Of the 58 patients with negative findings all proceeded to thoracotomy and complete resection was possible in 54 patients $(93.1 \%)$. We conclude that left anterior mediastinotomy is a valuable additional staging investigation for patients with tumours originating within the left upper lobe.

Thymoma: a comparison between surgical and pathological classification

K AL KATTAN, M SWEERTS, M SHEPPARD, P GOLDSTRAW Royal Brompton Hospital, London During the period of 13 years between January 1981 and December 199354 resections were performed for thymic tumours. A complete revision of the histological slides was available for 27 patients with true thymoma. Intraoperative clinical staging showed 14 in stage 1 , three in stage 2 , six in stage 3 , and four in stage 4. Average follow up period of 63 months (24-109) showed significant longer survival in clinical stage 1 than stages 3 and 4 (actuarial 10 year survival $91 \%$ compared with $66 \%$ and $50 \%$, respectively). The Rosai-Levine cellular staging and the Muller classification failed to correlate with long term survival compared with the Masaoka and operative clinical staging. Complete surgical resection showed a better survival. We conclude that the Masaoka and clinical staging is the best prognostic indicator; however, multicentre studies are needed to evaluate the other classifications. For true thymoma complete surgical resection should be performed to ensure long term survival.

Pleural lactic dehydrogenase (LD) and isoenzymes as additional diagnostic markers in pleural effusion

D DEV, J JOSEPH, MJ SMITH, SM VINEY, P BECK, GS BASRAN Respiratory Unit and Department of Biochemistry, Rotherham General Hospital NHS Trust, Rotherham, South Yorkshire Pleural fluid total LD analysis is being increasingly used in the USA and Europe in separating transudative from exudative pleural effusions. The further role of LD and its isoenzymes as an additional marker to aid in the differential diagnosis of pleural effusions has not been extensively explored. We have studied the biochemical profile of 180 patients with pleural effusions due to a variety of defined diagnoses with special reference to total LD (IU/1), LD isoenzymes, and total protein (TP). Total LD, $\mathrm{LD}$ fluid/serum (F/S) ratio and $\mathrm{LD}$ isoenzymes (1-5) F/S ratio were found to be minimum in cardiac failure (CCF) and maximum in empyema. The value was intermediate in malignancy and other exudative conditions as shown in the table (median concentrations)

\begin{tabular}{|c|c|c|c|c|c|}
\hline Group & $n$ & $\begin{array}{l}T P \\
F / S \text { ratio }\end{array}$ & $\begin{array}{l}\text { Total } \\
L D\end{array}$ & $\begin{array}{l}L D \\
F / S \text { ratio }\end{array}$ & $\begin{array}{l}L D-5 \\
F / S \text { ratio }\end{array}$ \\
\hline Cardiac failure & 37 & $0 \cdot 37$ & 95 & 0.4 & 0.79 \\
\hline Empyema & 17 & $0 \cdot 70$ & 3940 & $16 \cdot 7$ & $18 \cdot 9$ \\
\hline NMT & 65 & 0.61 & 281 & $1 \cdot 1$ & 1.4 \\
\hline Mesothelioma & 7 & 0.59 & 157 & 0.89 & $2 \cdot 8$ \\
\hline Pulm embolism & 20 & 0.60 & 292 & 1.0 & $2 \cdot 2$ \\
\hline Parapneumonic & 12 & 0.52 & 274 & 0.9 & 1.0 \\
\hline Others & 22 & 0.64 & 469 & 1.85 & $4 \cdot 2$ \\
\hline
\end{tabular}

The difference between CCF and non-CCF was significant $(p<0.001)$. Isoenzyme LD-5 ratio tended to be higher $(p<0.05)$ in pleural effusions of mesothelioma origin than in those from non-mesothelial tumours (NMT). Thus, for patients with cardiac failure presenting with pleural effusion, the finding of a total $\mathrm{LD}<100 \mathrm{IU} / 1$ combined with a TP $F / S$ ratio of $<0.4$ excluded other serious clinical conditions such as pulmonary embolism, parapneumonia, and malignancy as a cause of the effusion.
Differential pleural effusion kinetics in mesothelioma and carcinoma: a study using a dual isotope technique

D DEV, J JOSEPH, MJ SMITH, GS BASRAN Respiratory Unit, Rotherham General Hospital, Rotherham, South Yorkshire Pleural effusion results from an imbalance between the rate of formation and rate of removal of fluid from the pleural space. We have monitored the formation and drainage of pleural fluid in man using a radiolabelled protein technique (Nucl Med Commun 1992;13:432). Fifty three patients with free flowing pleural effusion from a variety of causes were investigated $\overrightarrow{\vec{F}}$ using this method. Fluid formation was monitored by measuring the $\overrightarrow{\mathcal{O}}$ rate of movement from blood into effusion of transferrin radiolabelled $\bar{C}$ in vivo with indium-113 $\mathrm{m}$ [Kin]. Fluid drainage [Kout] was estimated $\frac{\mathrm{\sigma}}{\mathrm{\sigma}}$ by measuring the rate of appearance in the blood of radiolabelled $\overline{\bar{\omega}}$ albumin (iodine-125) injected directly into the effusion. The Kin $\mathbb{\Phi}$ and Kout were calculated using a three compartmental model and expressed in the table as mean (SE) $\left(10^{-4} \mathrm{~h}^{-1}\right)$. Outflow (Kout) was

\begin{tabular}{|c|c|c|c|}
\hline Diagnosis & $n$ & Kin & Kout \\
\hline $\begin{array}{l}\text { Mesothelioma } \\
\text { Lung cancer } \\
\text { Met. cancer } \\
\text { Cardiac failure } \\
\text { Pancreatitis } \\
\text { Others }\end{array}$ & $\begin{array}{r}10 \\
18 \\
7 \\
8 \\
1 \\
9\end{array}$ & $\begin{array}{c}9.9(3 \cdot 2) \\
30 \cdot 1(12 \cdot 6) \\
39 \cdot 0(24 \cdot 1) \\
7.6(1.9) \\
114 \\
18.5(9.6)\end{array}$ & $\begin{array}{c}4 \cdot 7(0 \cdot 9) \\
24 \cdot 2(3 \cdot 5) \\
28 \cdot 0(7 \cdot 3) \\
35 \cdot 1(9 \cdot 9) \\
225 \\
53 \cdot 2(9 \cdot 7)\end{array}$ \\
\hline
\end{tabular}

significantly lower in effusions due to mesothelioma than primary lung cancer $(p<0.005)$, metastatic lung cancer $(p<0.001)$, or cardiac 을 failure $(\mathrm{p}<0.001)$. The inflow (Kin) was maximum in pancreatitis (Kin $\rightarrow$ of 114) consistent with increased vascular permeability/exudation. We speculate that, in mesothelioma, the predominant mechanism of effusion formation is the effect of the tumour on the lymphatic system, resulting in low Kout, even before the tumour produces the gross pleural encasement. In carcinomatous involvement of the pleura, however, the predominant mechanism appears to be a modest increase in rate of exudation due to increased vascular permeability. Because $\frac{O}{D}$ of the apparent difference in mechanism and consequently low values $\varrho$ of Kout, the isotope technique could form the basis of a simple $\overrightarrow{\overrightarrow{0}}$ methodology for distinguishing malignant pleural effusions associated 3 with mesothelioma from those associated with carcinoma - a common clinical dilemma.

Use of genetic markers to identify precancerous bronchial lesions in lung carcinogenesis

U PASTORINO, P GOLDSTRAW Royal Brompton Hospital, London In an attempt to define premalignant changes occurring in the multistep process of lung carcinogenesis, a cytogenetic and genetic study was performed at the National Cancer Institute of Milan on 94 patients undergoing pulmonary resection for an early stage lung cancer and 11 controls resected for other diseases. The panel of genetic markers $N$ included the assessment of chromosomal abnormalities, over- or expression of EGFR and HER2/NEU and p53 mutation in normal $N$ bronchial epithelium and primary tumour specimens. Of 94 cases, $N$ 40 displayed multiple synchronous or metachronous tumours in the $\sigma$ upper aerodigestive field, while 54 had either single tumours (45) or multiple tumours out of field (nine). Cytogenetic alterations were observed in $60 \%(28 / 47)$ of the evaluable tumour specimens with $\$$ complex rearranged karyotypes, particularly involving chromosomes $3(64 \%)$ and $17(36 \%)$. Gene alterations were detected including overexpression of the EGFR in $57 \%(46 / 80)$, HER2/NEU in $21 \%$ o (17/79), and p53 mutations in 47\% (23/49). The overall frequency of genetic changes (any type) in the tumour was $78 \%(73 / 94)$. In the normal bronchial mucosa we identified a rearranged karyotype in $\sigma$ $23 \%$ of the evaluable cases (20/86); particularly simple rearrangements involving chromosomes $3 \mathrm{p}$ (13 cases), 7 (six cases), and 17 (three $ᄋ$ cases), as well as overexpression of EGFR in 35\% (27/74) and of HER2/NEU in $15 \%(11 / 74)$. The overall frequency of genetic changes (any type) in the normal epithelium was $43 \%$ (39/91). The overall frequency of genetic changes in the normal epithelium was higher in patients with multiple tumours in the field (60\%) compared with those with single or multiple tumours in other sites (30\%). In addition, deletions of chromosome $3 \mathrm{p}$ and p53 mutations have been detected in precancerous lesions of the bronchial mucosa in five patients. These data indicate that various stages of lung tumorigenesis display specific genetic alterations and that a "field cancerisation" effect results in 
the appearance of detectable genetic lesions at sites distant from the tumour.

\section{Acute neurohormonal responses to hypoxaemia in humans}

RI CARGILL, LC MCFARLANE, WJ COUTIE, BJ LIPWORTH Department of Clinical Pharmacology, Ninewells Hospital and Medical School, Dundee Whilst the acute pulmonary pressor response to hypoxaemia in man is well documented, there remains some controversy regarding the effects of this stimulus on integrated hormonal responses, particularly on activity of the renin-angiotensin-aldosterone system (RAAS). This may be important in the adaptive response to alveolar hypoxia, either in pulmonary disease or adverse environmental conditions. These neurohormonal changes have not been fully characterised nor studied in association with the haemodynamic effects of hypoxaemia. These responses were therefore studied in 10 healthy volunteers on two separate occasions. After reaching a resting haemodynamic state, subjects breathed for 30 minutes either room air or a nitrogen/oxygen mixture which rendered $\mathrm{SaO}_{2}$ between $75 \%$ and $80 \%$. Pulmonary and systemic haemodynamic parameters were measured and venous blood samples taken at baseline and after 30 minutes breathing air or the hypoxic gas. Hypoxaemia significantly increased heart rate, cardiac output, and mean pulmonary artery pressure (MPAP), but not mean arterial pressure (MAP) compared with normoxaemia. Although plasma renin activity (PRA) and angiotensin II were unaffected by hypoxaemia, plasma aldosterone (Aldo) fell significantly in comparison with normoxaemia. There was a nonsignificant increase in plasma levels of cortisol and catecholamines during hypoxaemia but no changes were observed during normoxaemia (table). Thus, the renin-angiotensin system becomes dis-

\begin{tabular}{llcl}
\hline & Normoxaemia & Hypoxaemia & $p$ \\
\hline$\Delta$ MPAP (mm Hg) & $-0.2(0.5)$ & $18.2(1.5)$ & $<0.05$ \\
$\Delta$ MAP (mm Hg) & $-0.5(1 \cdot 6)$ & $4 \cdot 4(2 \cdot 2)$ & NS \\
$\Delta$ PRA (pmol/ml/min) & $-0.13(0.11)$ & $-0.04(0.09)$ & NS \\
$\Delta$ Aldo (pmol/l) & $-1.4(7.5)$ & $-35.7(8.9)$ & $<0.05$ \\
\hline
\end{tabular}

sociated during hypoxaemia where plasma aldosterone levels decreased despite having no significant effects on other components of the RAAS or other markers of adrenal cortical and medullary function. This dissociation may be influenced by the counter-regulatory effects of the natriuretic peptides released under hypoxic conditions.

\section{Effect of acute changes in oxygen tension on responses to} methacholine in bronchi from chronically hypoxic rats

RA ClAyTON, JE NALLY, MR MACLEAN, NC THOMSON, JC MCGRATH Institute of Biomedical and Life Sciences, Glasgow University; Department of Respiratory Medicine, Western Infirmary, Glasgow Sustained alveolar hypoxia is a feature of airway diseases such as chronic obstructive pulmonary disease. We have previously shown in bovine bronchi (Clayton et al. Br F Pharmacol 1994;112:593P) that acute changes in $\mathrm{O}_{2}$ levels alter reactivity to methacholine (MCh); however, it remains to be seen whether chronic hypoxia alters the sensitivity of bronchi to acute changes in $\mathrm{O}_{2}$ tension in the rat. We examined contractions of chronically hypoxic and control rat bronchi to $\mathrm{MCh}$ at $95 \%, 20 \%$, and $4 \% \mathrm{O}_{2}$. Rats were reared in a hypoxic/hypobaric chamber for 14 days under a pressure of 500-550 millibars, giving effective gas concentrations of $10 \% \mathrm{O}_{2}, 0.03 \% \mathrm{CO}_{2}$, and balance $\mathrm{N}_{2}$. Rats were sacrificed and contractions of rings of first order bronchi were measured isometrically ( $n=6$ in each case). Cumulative concentration response curves were constructed to $\mathrm{MCh}$ $\left(10^{-8}-3 \times 10^{-4} \mathrm{M}\right)$, results being expressed as \% of the maximum response in $95 \% \mathrm{O}_{2}$. In control rats there was no difference between responses in $95 \%$ and $20 \% \mathrm{O}_{2}$, however, responses in $4 \%$ were significantly $(p<0.001)$ attenuated, with a mean $29.3 \%$ decrease in maximum response. Bronchi from hypoxic rats showed a similar pattern; with responses in $4 \%$ being significantly $(p<0.05)$ attenuated with a mean $25.6 \%$ decrease in maximum response. Exposure to chronic hypoxia therefore does not appear to alter sensitivity to acute changes in $\mathrm{O}_{2}$ tension in rat bronchi. [Supported by the Chest Heart and Stoke Association (Scotland), the Wellcome Foundation, and the National Asthma Campaign.]
Effect of hypoxia and $\beta_{2}$ agonists on the activity of the reninangiotensin system

EA MILLAR, JE NALLY, RM ANGUS, RA CLAYTON, NC THOMSON Department of Respiratory Medicine, Western Infirmary, Glasgow We have reported that the renin-angiotensin system (RAS) is activated in acute severe asthma and also by $\beta_{2}$ agonists. Another possible stimulus to the activation of the RAS during acute asthma is hypoxia, although previous studies regarding the effect of hypoxia on the RAS have produced conflicting results. The present study examined the effect of hypoxia alone and in combination with $\beta_{2}$ agonist therapy on plasma renin and angiotensin II (AII) levels. On four separate study days in a double blind, placebo controlled crossover study eight healthy volunteers were randomised to receive either hypoxic mixture $\left(\mathrm{FIO}_{2} 12 \%\right)$ or air for a period of 30 minutes, with either salbutamol ( $5 \mathrm{mg}$ ) or placebo $(3.5 \mathrm{ml} \mathrm{N}$ saline) administered into the circuit via a nebuliser (at time $\mathrm{t}=10 \mathrm{~min}$ ). Oxygen saturation was monitored throughout the study by pulse oximeter and blood withdrawn at baseline, $10,20,30,45,60,90$, and 120 minutes for measurement of plasma renin and AII. There was no significant difference between baseline values of renin or AII on either study day. Following the period of hypoxia alone [mean (SE) $\mathrm{O}_{2}$ saturation $82 \cdot 8(1 \cdot 5), 87 \cdot 6$ (3.9), $84.5(3.0) \%$ at 10,20 and 30 minutes] there were no significant changes in plasma levels of renin or AII. When salbutamol was added to the hypoxic mixture, there were significant rises in plasma renin and AII levels [mean (SE) increase in AII from baseline of 7.2 (4.9), $4.5(3.4), 5 \cdot 4(2.9), 3.6(3 \cdot 1) \mathrm{pg} / \mathrm{ml}$ at $30,45,60$, and 90 minutes, and in renin of $14 \cdot 1(6 \cdot 5), 12 \cdot 8(4 \cdot 8), 15 \cdot 5(6 \cdot 3), 4 \cdot 0(3 \cdot 2)$ and $4.0(3.9) \mu \mathrm{U} / \mathrm{ml}$ at $30,45,60,90$, and 120 minutes, respectively]. Nebulised salbutamol in air also increased plasma renin and AII levels, but there was no significant difference between the effect of salbutamol in either the normoxic or hypoxic mixture. We conclude that there is activation of the RAS by salbutamol, but not by hypoxia. Hypoxia does not influence the effect of salbutamol on the RAS. Thus, $\beta_{2}$ agonists, but not hypoxia, are likely to be contributing to the activation of the RAS in acute asthma. [Supported by the National Asthma Campaign.]

Angiotensin II (AII) potentiates, in vitro and in vivo, methacholine (MCh)-induced bronchoconstriction

JE NALLY, EA MILLAR, NC THOMSON, JC MCGRATH Insitute of Biomedical and Life Sciences, University of Glasgow; Department of Respiratory Medicine, Western Infirmary, Glasgow Plasma AII levels are raised in patients with acute severe asthma and also by $\beta$ agonists. In addition, intravenous AII evokes bronchoconstriction in mild asthmatic patients. This study examined the effects of AII on bronchi in vitro and the interaction of AII with $\mathrm{MCh}$-evoked contractions both in vivo and in vitro. Contractions of rings of human and bovine bronchi were measured isometrically. Cumulative concentration-response curves were obtained to AII $\left(10^{-9}-10^{-6} \mathrm{M}\right)$. In addition, seven asthmatic patients with mild bronchial hyperreactivity to $\mathrm{MCh}$ were studied. In a randomised double blind study, patients received placebo or AII 1 or $2 \mathrm{ng} / \mathrm{kg} / \mathrm{min}$ by intravenous infusion. These doses were below those which evoke bronchoconstriction. At the end of the infusion $\mathrm{MCh}$ challenge was undertaken. $\mathrm{FEV}_{1}$ values were measured at baseline, at the end of the infusion, and following each $\mathrm{MCh}$ dose. $\mathrm{PC}_{20}$ results were expressed as geometric mean and range. AII alone in vitro evoked only small $(<0 \cdot 25 \mathrm{~g})$ contractions of human and bovine bronchi (threshold for contraction, $3 \times 10^{-8}-3 \times 10^{-7} \mathrm{M}$ ). Preincubation with AII $10^{-7} \mathrm{M}$ significantly $(\mathrm{p}<0.001)$ enhanced contractions to $\mathrm{MCh}$ with an increased maximum of $39.1 \%$ in human and $13.7 \%$ in bovine bronchi ( $n=6$ in each case). In mild asthmatic patients AII alone evoked no change in baseline $\mathrm{FEV}_{1}$ values. The geometric mean $\mathrm{PC}_{20}$ of $\mathrm{MCh}$ after placebo infusion was $3.09 \mathrm{mg} / \mathrm{ml}$ (range $1.15-6.0 \mathrm{mg} / \mathrm{ml}$ ). After infusion with AII $1 \mathrm{ng} / \mathrm{kg} / \mathrm{min}$ this was $2.14 \mathrm{mg} / \mathrm{ml}$ (range $0.85-3.8 \mathrm{mg} / \mathrm{ml}$ ). In the presence of AII $2 \mathrm{ng} / \mathrm{kg}$ / min there was a significant $(\mathrm{p}=0.006)$ decrease in $\mathrm{PC}_{20}$ compared with placebo (geometric mean, $1.2 \mathrm{mg} / \mathrm{ml}$, range $0.45-2.08 \mathrm{mg} / \mathrm{ml}$ ). In conclusion, AII in subthreshold concentrations enhances $\mathrm{MCh}$ evoked bronchoconstrictions both in vitro in human and bovine tissue, and also in vivo in mild asthmatic patients. [This work was supported by the National Asthma Campaign.]

Lisinopril inhibits elevation of plasma angiotensin II by nebulised $\beta_{2}$ agonists

EA MILLAR, GT MCINNES, NC THOMSON Department of Respiratory Medicine, Western Infirmary, Glasgow We have described activation 
of the renin-angiotensin system (RAS) by high dose nebulised $\beta_{2}$ agonists and have now examined whether this effect is influenced by pretreatment with ACE inhibitor drugs. On four separate days in a randomised double blind crossover study design eight healthy volunteers were pretreated with either lisinopril $(20 \mathrm{mg})$ or identical placebo capsules. Three hours later an intravenous cannula was inserted into a forearm vein for blood sampling, followed by a short rest. Each subject then received either nebulised salbutamol $(5 \mathrm{mg})$ or placebo inhalation $(3.5 \mathrm{ml} 0.9 \%$ saline), so that subjects received all possible combinations of treatment after four study visits. At baseline and at $15,30,45,60,90$, and 120 minutes after nebulisation blood was withdrawn for estimation of plasma levels of renin and angiotensin II (AII). Serum ACE was measured only at baseline and at 120 minutes. Baseline plasma renin levels were significantly raised on both lisinopril treatment days when compared with placebo, and did not alter significantly after placebo inhalation. There were significant increases in plasma renin levels after salbutamol when preceded by placebo or lisinopril (mean (SE) baseline renin of 35.6 $(8 \cdot 3)$ and $160 \cdot 1(20.6) \mu \mathrm{U} / \mathrm{ml}$ increasing to $61 \cdot 7(15 \cdot 6)$ and $198 \cdot 4$ $(18.9) \mu \mathrm{U} / \mathrm{ml}$, respectively, at 15 minutes, $\mathrm{p}<0.05 v$ placebo inhalation). Baseline plasma AII level was significantly lower on both lisinopril days when compared with placebo pretreatment. Salbutamol produced a significant rise in AII when preceded by placebo capsules (mean (SE) baseline AII of $8.2(2 \cdot 2) \mathrm{pg} / \mathrm{ml}$ increasing to $17 \cdot 7(5.4) \mathrm{pg} /$ $\mathrm{ml}$ at 15 minutes, $\mathrm{p}<0.05 v$ placebo). However, pretreatment with lisinopril prevented this rise in AII (mean (SE) baseline AII of 1.4 $(0.1) \mathrm{pg} / \mathrm{ml}$ and $1.5(0.4) \mathrm{pg} / \mathrm{ml}$ at 15 minutes) and AII did not alter significantly at any time point thereafter. In all subjects serum ACE levels on both lisinopril days were suppressed, both prior to, and on completion of, each study visit. These results confirm that high dose $\beta_{2}$ agonists activate the RAS. In addition, pretreatment with ACE inhibitors can prevent the rise in plasma AII by these agents, indicating that this action of $\beta_{2}$ agonists is mediated via the classical RAS pathway involving ACE. [Supported by the National Asthma Campaign.]

\section{Role of potassium channels in the bronchodilator effect of atrial natriuretic peptide in bovine bronchi}

JE NALLY, CC DOCHERTY, NC THOMSON Institute of Biomedical and Life Sciences, University of Glasgow; Department of Respiratory Medicine, Western Infirmary, Glasgow ANP ( $\alpha$-human atrial natriuretic peptide) is a 28 amino acid hormone which evokes bronchodilation in human and bovine bronchi (Angus et al. Clin Sci 1994;86:291-5). The mechanism underlying this response is, however, unknown. The present study examined the role of potassium channels in the bronchodilation evoked by this peptide in bovine bronchi. Contractions were evoked by methacholine $\left(3 \times 10^{-6} \mathrm{M}\right)$. Once a plateau had been reached, cumulative concentration-response curves were obtained for ANP $\left(10^{-9}-3 \times 10^{-6} \mathrm{M}\right.$ in the presence of phosphoramidon $3.7 \times 10^{-5} \mathrm{M}$ to prevent hydrolysis) in the presence and absence of tolbutamide $\left(10^{-7}-10^{-5} \mathrm{M}\right)$, apamin $\left(10^{-8}-10^{-6} \mathrm{M}\right)$, or charybdotoxin $\left(\mathrm{ChTx}, 10^{-9}-10^{-7} \mathrm{M}\right)$. The ATP-sensitive $\mathrm{K}^{+}$channel blocker tolbutamide $\left(10^{-6}\right.$ and $\left.10^{-5} \mathrm{M}\right)$ attenuated the relaxations induced by ANP $\left(-\log\right.$ IC $_{50}, 5.98(0.64)$ and $6.02(0.9)$, respectively, compared with control $6.92(0 \cdot 17), \mathrm{p}<0 \cdot 01, \mathrm{n}=6)$. Tolbutamide $10^{-7} \mathrm{M}$ was ineffective. Blockade of small conductance $\mathrm{Ca}^{2+}$-activated $\mathrm{K}^{+}$ channels with apamin $\left(10^{-8}-10^{-6} \mathrm{M}, \mathrm{n}=5\right)$ had no significant effect on ANP-induced relaxations. Neither tolbutamide nor apamin altered baseline tone. ChTx $10^{-7} \mathrm{M}$ alone evoked a marked increase in the baseline tone, indicative of large conductance $\mathrm{Ca}^{2+}$-activated $\mathrm{K}^{+}$ channels being open under resting conditions. ChTx $\left(10^{-9}-10^{-7} \mathrm{M}\right)$ had no effect, however, on responses to ANP $(n=5)$. In conclusion, the bronchodilation evoked by ANP in bovine bronchi appears to involve the opening of ATP sensitive, but not large or small conductance $\mathrm{Ca}^{2+}$-activated, $\mathrm{K}^{+}$channels. [Supported by the Chest Heart and Stroke Association (Scotland) and the National Asthma Campaign.]

\section{Effect of thiorphan, an inhibitor of neutral endopeptidase, on the bronchodilator response to inhaled atrial natriuretic peptide in asthma}

RM ANGUS, EA MILLAR, GW CHALMERS, NC THOMSON Department of Respiratory Medicine, Western Infirmary, Glasgow The hormone atrial natriuretic peptide (ANP) causes bronchodilation and partially protects against direct and indirect bronchial challenges. ANP is thought to be inactivated by two routes: degradation by the enzyme neutral endopeptidase-24.11 (NEP) and binding to a non-guanylyl cyclase clearance receptor. We have found in studies on human airway that phosphoramidon, whose actions include inhibition of NEP, enhances the in vitro protective effect of ANP on methacholine induced contraction. Similarly in vivo thiorphan, an inhibitor of NEP, enhances the protective effect of ANP against histamine-induced bronchoconstriction. We therefore hypothesised that pretreatment with thiorphan might enhance the bronchodilator response to inhaled ANP. In a randomised, double blind, placebo controlled, crossover study, six asthmatic patients (one woman), mean (SD) age $47 \cdot 3(3 \cdot 8)$ years and $\mathrm{FEV}_{1} 1.91(0.42) 1,55(3.8) \%$ predicted, were studied. All were shown at screening to have at least a $25 \%$ improvement in $\mathrm{FEV}_{1}$ to salbutamol. Patients were asked to withhold $\beta_{2}$ agonists for eight $\frac{\bar{\sigma}}{\bar{\sigma}}$ hours while inhaled corticosteroids were continued unaltered. On $\overline{\bar{S}}$ study visits they received either thiorphan $1 \mathrm{mg}$ (in $2 \mathrm{ml}$ ) followed by ANP $5 \mathrm{mg}$ or placebo (saline) or placebo (saline) followed by ANP $(5 \mathrm{mg})$, placebo or salbutamol $5 \mathrm{mg}$. Spirometric values were meas- $\infty$ ured after each inhalation then followed for two hours. ANP alone $\overrightarrow{0}$ caused a significant bronchodilator response at 10 and 15 minutes when compared with placebo and thiorphan alone, mean (SE) \% $\vec{\omega}$ change in $\mathrm{FEV}_{1}$ of $16.8(8 \cdot 1)$ and $16 \cdot 1(6 \cdot 8)$, respectively. Prior inhalation of thiorphan prolonged bronchodilator effect of ANP, $\overrightarrow{\times}$ significant bronchodilatation being noted at five minutes (mean (SE) $\%$ change in $\mathrm{FEV}_{1}$ of $17.5(1.2)$ ) and being maintained up to 60 minutes $(23 \cdot 1(3 \cdot 4))$. With both ANP and the combination of the NEP inhibitor and ANP the degree of bronchodilatation was significantly less than that produced by salbutamol which was maximal $\vec{\tau}$ at one hour (mean (SE) \% change in $\mathrm{FEV}_{1}$ of $53(10.5)$ ) and 음 maintained at two hours. These results confirm that airway NEP is important in modulating the effect of inhaled ANP. [Supported by UCB and the British Lung Foundation.]

Bronchodilator responsiveness to salbutamol and histamine reactivity after continuous treatment with twice daily salmeterol

A GROVE, RA ClARK, JH WINTER, DP DHILlON, LC MCFARLANE, DG MCDEVITT, BJ LIPWORTH Department of Clinical Pharmacology and Respiratory Medicine, University of Dundee Medical School, Ninewells $\overrightarrow{\overrightarrow{0}}$ Hospital, Dundee Seventeen asthmatics, all receiving inhaled steroids, 3 of mean (SD) age 34 (3) years and $\mathrm{FEV}_{1} 62$ (3)\% of predicted, were evaluated. After a two week run in without $\beta_{2}$ agonists they received salmeterol (SMT) dry powder $50 \mu \mathrm{g}$ bd or placebo (PL) for four weeks in a randomised double blind crossover design. Twelve hours after the last dose of each treatment a histamine test was performed and after 36 hours a salbutamol DRC was constructed. Airway and systemic baselines were not significantly different for SMT $v$ PL. There was a parallel right shift in DRC after SMT $v$ PL for PEFR $(p=0.036)$ and $\mathrm{FEV}_{1}(\mathrm{p}=0.058)$, but not for $\mathrm{FEF}_{25-75}$. The maximal bronchodilator delta response was not, however, attenuated: FEV $_{1}$ : SMT $0.591 v$ PL 0.701 (95\% CI -0.02 to 0.241 ), PEFR: SMT 음 $100 \mathrm{l} / \mathrm{min} v \mathrm{PL} 116 \mathrm{l} / \mathrm{min}(95 \% \mathrm{CI}-6$ to $38 \mathrm{l} / \mathrm{min})$. The $\mathrm{FEV}_{1} \mathrm{PC}_{20}$ (log) following SMT was $0.37 \mathrm{mg} / \mathrm{ml} v$ PL $0.20 \mathrm{mg} / \mathrm{ml}, \mathrm{p}<0.05(95 \%$ 을 CI 0.002 to 0.334$)$. Systemic responses were attenuated after SMT
PL (p<0.05): potassium: SMT $-0.73 \mathrm{mmol} / 1 v \mathrm{PL}-1.03 \mathrm{mmol} / 1 \mathrm{~J}$ ( $95 \%$ CI -0.055 to $-0.05 \mathrm{mmol} / \mathrm{l})$. Lymphocyte $\beta_{2}$ density (log) N tended to be lower after SMT: $0 \cdot 22 \mathrm{fmol} / 10^{6}$ cells compared with PL: N $0.31 \mathrm{fmol} / 10^{6}$ cells, or after run in: $0.31 \mathrm{fmol} / 10^{6}$ cells (NS). AM W PEFR was improved during treatment with SMT $v$ PL $(\mathrm{p}<0.05):$ ㅇ $421 \mathrm{l} / \mathrm{min} v 395 \mathrm{l} / \mathrm{min}(95 \%$ CI 6 to $46 \mathrm{l} / \mathrm{min})$. In summary, SMT $50 \mu \mathrm{g}$ bd produced a right shift in salbutamol DRC but did not blunt the maximal bronchodilator response, resulted in a small but significant protection against histamine reactivity, significantly blunted $T$ maximal systemic $\beta_{2}$ responses to salbutamol, and showed a trend to reduced lymphocyte $\beta_{2}$ adrenoceptor density.

Effect of cyclosporin A (CsA) on the expression of activation markers by $T$ lymphocytes in chronic severe asthma

SH LOCK, CJ CORRIGAN, NC BARNES, AB KAY Royal Brompton National 윽 Heart and Lung Institute, Department of Allergy and Clinical Immunology, London; The London Chest Hospital, London Corrigan et al found that elevated numbers of peripheral blood $T$ lymphocytes express activation markers in acute severe asthma and the reduction of expression after therapy could be correlated with improvement in lung function ( $\mathrm{Am}$ Rev Respir Dis 1990;141:970-7). We have studied the expression of activation markers by peripheral blood $\mathrm{T}$ lymphocytes 
in 39 corticosteroid dependent asthmatics taking part in a double blind, placebo controlled corticosteroid reduction study using CsA. Nineteen patients received CsA at $5 \mathrm{mg} / \mathrm{kg} /$ day and 20 received identical placebo. Mean (SE) blood levels in the CsA group were 144 $(7 \cdot 2) \mathrm{ng} / \mathrm{ml}$. Peripheral blood mononuclear cells were isolated from patients at baseline and after 24 weeks trial medication using Ficoll gradients. Expression of activation markers (HLA-DR, CD25) on CD4 $\mathrm{T}$ lymphocytes were measured using flow cytometry. There was no significant difference in activation marker expression between the cyclosporin and placebo groups at baseline. Aftr 24 weeks of trial medication there was a significant reduction in HLA-DR (median $13 \%, p=0.01)$ and $\operatorname{CD} 25(-37 \%, p<0.01)$ expression on CD4 T lymphocytes in the CsA compared with the placebo group. CsA reduces the expression of activation markers on peripheral blood $\mathrm{T}$ lymphocytes from asthmatics and this may be central to the mechanism of action of CsA in asthma.

\section{MRI cerebral white matter changes in obstructive sleep apnoea} (OSA)

RJO DAVIES, S RENOWNDEN, N MOORE, JR STRADLING Osler Chest Unit and Radiology Department, Oxford Radcliffe Hospital, Oxford Patients with obstructive sleep apnoea suffer an excess of cerebrovascular disease, but this might be due to many confounding risk factors seen in these patients, particularly obesity. Cerebral magnetic resonance imaging (MRI) allows the identification of clinically silent cerebral ischaemic demyelination which is seen as small areas of white matter high signal. To assess whether such silent ischaemia is more frequent in OSA patients than in controls matched for the major confounding variables, eight patients with OSA and 10 control subjects underwent MRI. The study groups did not differ for any of the following indices: sex, age, body mass index, alcohol and tobacco intake, daytime ambulatory blood pressure, waist to hip ratio, and fasting lipid concentrations. The patients with OSA had their obstructive sleep apnoea conformed by full polysomnography ( $>4 \% \mathrm{SaO}_{2}$ dip rate $>15 /$ hour). The controls were normal community subjects. All subjects underwent T1 and T2 weighted MRI imaging. These images were performed and interpreted blind of the patient/control status. The presence of white matter high signal abnormalities was scored at three levels: (1) no abnormality, (2) 1-5 abnormal foci, (3) $>5$ abnormal foci (table).

\begin{tabular}{llll}
\hline & \multicolumn{3}{l}{ Number of abnormal high signal foci } \\
\cline { 2 - 4 } & None & $1-5$ & $>5$ \\
\hline OSA patients & 5 & 1 & 2 \\
Controls & 4 & 1 & 5 \\
\hline
\end{tabular}

Both the patients with OSA and the matched control subjects showed frequent cerebral ischaemic lesions consistent with their generally unhealthy state. These were no more prevalent in the patient group than the control subjects.

Nasal CPAP as a treatment for obstructive sleep apnoea: objective versus subjective compliance

JM PARTLETT, DJ PITSON, S BUCK, JR STRADLING Osler Chest Unit, Churchill Hospital, Oxford Nasal continuous positive airway pressure (CPAP) successfully treats obstructive sleep apnoea (OSA). Data from the US (Kribbs et al. Am Rev Respir Dis 1993;147:887) has suggested poor compliance measured objectively under 25 hours/ week. It is not clear whether compliance needs to be measured or whether patients report usage accurately. This study was carried out on 71 patients using CPAP over an average period of 25 months (range 9-60). The patients were unaware that the clocks were being read for the study. Subjective compliance was collected as part of a validated questionnaire survey (Hoffstein et al. Am Rev Respir Dis 1992;145:841) which allowed comparison with this Canadian group. Mean (SD) objective compliance in our patients was $41.2(11.7)$ hours/week (5.9 hours/night), and subjective compliance was 49.6 (9.3) hours/week (7.0 hours/night), compared with $33 \cdot 2$ hours/week in the Canadian study. On average, our patients overreported their usage by $8.35(95 \%$ CI 5.7 to 11.0$)$ hours/week. The graph illustrates the relationship between subjective and objective compliance. A subgroup $(n=21)$ had two timeclock readings one year apart; mean

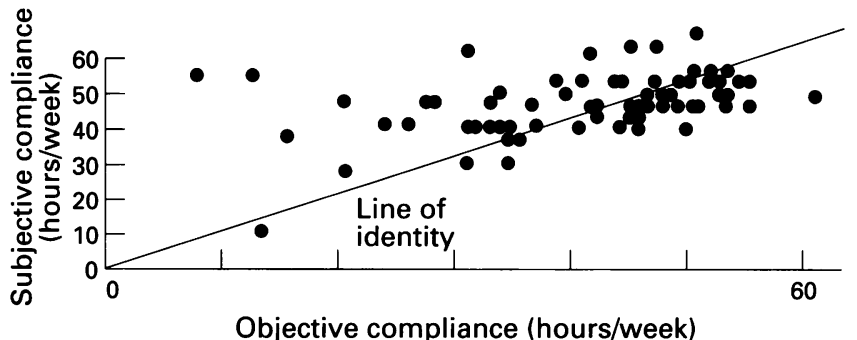

(SD) objective compliance rose from $45 \cdot 2(8 \cdot 8)$ to $50 \cdot 4(7 \cdot 4)$ hours/ week in the subsequent year. On average, patients overestimated their usage by an hour per night and there is an overall increase in objective CPAP compliance with time.

Variation in CPAP requirement using an intelligent CPAP device

N POTTER, AK SIMmONDS Royal Brompton Hospital, London Although conventional continuous positive airway pressure (CPAP) machines deliver a constant pressure throughout the night, the pressure required to maintain airway patency in any individual with obstructive sleep apnoea (OSA) may vary depending on a number of factors including sleep stage and body posture. The Sullivan Autoset Clinical system (Rescare) has been developed to titrate CPAP level to nasal airflow. It has been used in this pilot study to assess the individual variation in overnight CPAP requirement in 17 patients $(15 \mathrm{men})$ with baseline apnoea/hypopnoea index (AHI) of 33 (range 7-91). In eight patients sleep staging was carried out during the autoset study and body position monitored by video recording. For the group as a whole median (SD) CPAP requirement was $8 \cdot 1(1 \cdot 2) \mathrm{cm} \mathrm{H}_{2} \mathrm{O}$ with a 90th centile level of $10.9(1.6) \mathrm{cm} \mathrm{H}_{2} \mathrm{O}$. Mean (SD) apnoea index determined by the autoset was $4(4 \cdot 8)$. There was, however, a wide range in CPAP requirement within individuals overnight. In some patients CPAP level was related to sleep stage, rising in REM sleep, in others changes in sleep stage had no consistent effect. Two groups were identified: one with a $<2 \mathrm{~cm} \mathrm{H}_{2} \mathrm{O}$ variation between median and 90th centile CPAP level and the other with a $>2 \mathrm{~cm} \mathrm{H}_{2} \mathrm{O}$ variation between these values. Body mass index did not differ significantly between the groups but the group with the greater variation in overnight CPAP requirement had a higher baseline AHI (50 v 39, $p=0.01$ ) indicating more severe disease. The consequences of failing to accurately titrate CPAP are unknown, but there does seem to be a subgroup of OSA patients with a highly variable CPAP requirement which might benefit from more flexible overnight treatment.

Use of the Epworth Sleepiness Scale (ESS) to monitor response to treatment with nasal continuous positive airways pressure (nasal CPAP) in patients with obstructive sleep apnoea

FM HARDINGE, D PITSON, JR STRADLING Osler Chest Unit, Churchill Hospital, Oxford The ESS (Johns. Sleep 1991;14:50-5) is a questionnaire derived scale of sleepiness which can be used to assess the degree of daytime sleepiness suffered by patients with obstructive sleep apnoea (OSA). Its use has been well validated in a variety of sleep conditions. When patients with OSA are treated with nasal CPAP their subjective sleepiness often markedly improves. But objective tests of daytime vigilance such as the multiple unprepared reaction time test or multiple sleep latency test have demonstrated significant but often only very small improvements following treatment with nasal CPAP. We administered ESS questionnnaires to patients at initial assessment and at two months after commencing nasal CPAP in one group (48 patients, mean age $50 \cdot 2$ years, $94 \%$ men), and after one year of treatment with nasal CPAP in a second group (18 patients, mean age 49.7 years, all men). In the first group mean (SE) ESS before treatment was $16(0.5)$ and after two months treatment had fallen to $8(0.6)(p=0.0001)$. In the second group mean ESS before treatment was $14(1 \cdot 3)$ and after treatment for one year had fallen to $6(0.9)(p=0.0001)$. In this second group compliance with treatment was monitored in 12 patients using Respironics machines with time clocks of which patients were unaware. Mean (SE) compliance was $6.54(0.52)$ hours/night. We conclude that changes in the ESS following treatment with nasal CPAP are more representative of the magnitude of patients' subjective response to treatment than other 
objective tests of daytime vigilance, possibly because the ESS is measuring a different component of sleepiness. It is therefore likely that the ESS could be useful clinically to monitor progress during treatment.

Use of pulse transit time as a measure of inspiratory effort in obstructive sleep apnoea

DJ PITSON, A SANDELl, R VAN DEN HOUT, JR STRADLING Osler Chest Unit, Churchill Hospital, Oxford Pulse transit time (PTT) is the time taken for the arterial pulse shock wave to travel from the aortic valve to the periphery. PTT is inversely proportional to blood pressure and it has been shown that, like systolic blood pressure swings, the size of the inspiratory swings in PTT correlate well with the degree of inspiratory effort in awake normal subjects breathing through an added threshold inspiratory valve. This study investigated the ability of inspiratory PTT swings to act as a measure of inspiratory effort in patients with obstructive sleep apnoea (OSA). Recordings were made on eight men of mean age 44 (range 34-58) years with OSA (mean $>4 \% \mathrm{SaO}_{2}$ dip rate 43 , range $13-68$ dips/hour) during their initial titration of nasal continuous positive airway pressure (NCPAP) NCPAP was decreased from optional treatment pressure to minimum pressure at three minute intervals in steps of $1 \mathrm{~cm} \mathrm{H}_{2} \mathrm{O}$ to produce a range of inspiratory efforts. Oesophageal pressure (IOP) and PTT were included in the standard sleep monitoring. About 20 breaths at each NCPAP level were measured for each patient. IOP swings were grouped together in sampling bins of $5 \mathrm{~cm} \mathrm{H}_{2} \mathrm{O}$ (range 0 to $-65 \mathrm{~cm} \mathrm{H}_{2} \mathrm{O}$ ) and the mean and standard error of the PTT swings within each bin was calculated. The slopes, intercepts and $r$ values are shown in the table. The correlation between PTT swings and

\begin{tabular}{llcl}
\hline Subject & Slope & Intercept & $r$ \\
\hline 1 & 0.40 & 7.7 & 0.99 \\
2 & 0.34 & 1.2 & 0.99 \\
3 & 0.33 & 7.5 & 0.94 \\
4 & 0.39 & $7 \cdot 0$ & 0.96 \\
5 & 0.38 & $10 \cdot 1$ & 0.90 \\
6 & 0.38 & $7 \cdot 1$ & 0.88 \\
7 & 0.60 & 3.7 & 0.96 \\
8 & 0.28 & 3.9 & 0.89 \\
Mean & 0.39 & 5.9 & 0.94 \\
\hline
\end{tabular}

IOP swings was high, therefore inspiratory PTT swings may be a useful indirect measure of inspiratory effort in patients with OSA

\section{Comparative study of cost of polysomnography and oximetry} in the sleep apnoea syndrome

M MAYOS, JL DIEZ BETORET, L HERNANDEZ, P CASAN, J SANCHIS Unitat Funció Pulmonar, Department de Pneumologia, Hospital Sta Creu i de St Pau; Servei Català de la Salut, Barcelona, Spain In the sleep apnoea syndrome (SAS) polysomnography (psm) is the most widely used diagnostic test. Oximetry (pox) has been proposed as an alternative test which simplifies diagnosis. Its sensitivity (S) and specificity (SP) vary according to the analytical criterion used. A study was performed to compare the financial cost of diagnosing SAS in patients by means of three defined diagnostic strategies: (A) polysomnography alone; (B) oximetry interpreted with criteria of maximum specificity, plus polysomnography if negative; (C) oximetry interpreted with criteria of maximum sensitivity, plus polysomnography if positive. The financial analysis took into account the costs of installation, maintenance and replacement of material, and staff costs. Theoretical values of S, SP and prior probability of suffering from SAS (P) were used. The average cost of diagnosing SAS $\left(\mathrm{C}_{\mathrm{SAS}}\right)$ was calculated by $\mathrm{C}_{\mathrm{SAS}}=\mathrm{C}_{\mathrm{psm}}$ $\mathrm{P}$ for method $\mathrm{A}$; by $\left.\mathrm{C}_{S A S}=\mathrm{C}_{\mathrm{pox}}+[(1-\mathrm{S}) \mathrm{P}+(1-\mathrm{P}) \mathrm{E}] \mathrm{C}_{\mathrm{psm}}\right) / \mathrm{P}$ for $\mathrm{B}$; by $\left.\mathrm{C}_{\mathrm{SAS}}=\mathrm{C}_{\mathrm{pox}}+[(1-\mathrm{P})(1-\mathrm{E})+\mathrm{PS}] \mathrm{C}_{\mathrm{psm}}\right) / \mathrm{P}$ for $\mathrm{C}$. The results are shown in the table. We conclude that, in the combinations of $S, S P$ and $P$

\begin{tabular}{llll}
\hline$P$ & $C_{S A S} A$ & $C_{S A S}{ }^{*} B$ & $C_{S A S}{ }^{*} C$ \\
\hline & & $\mathrm{S}=0.3, \mathrm{SP}=0.9$ & $\mathrm{~S}=0.9, \mathrm{SP}=0.3$ \\
0.3 & $£ 1574$ & $£ 1393$ & $£ 942$ \\
0.5 & $£ 944$ & $£ 798$ & $£ 798$ \\
0.7 & $£ 674$ & $£ 528$ & $£ 597$ \\
\hline
\end{tabular}

* Oximetry at outpatients. In cases of hospitalisation, $\mathrm{B}$ and $\mathrm{C}$ are greater than $\mathrm{A}$. analysed, oximetry at outpatients followed by polysomnography offers a lower financial cost than generalised polysomnography. In a selected population method $\mathbf{B}$ is preferable; in a non-selected population method $\mathrm{C}$ is the best option.

Comparison of laboratory polysomnography with home use of
the Edentrace II recorder in the diagnosis of sleep apnoea

SP FINCH, IL MORTIMORE, NL DOUGLAS Sleep Laboratory, Royal In- $\frac{\overline{\vec{\rho}}}{\stackrel{\rho}{ }}$ firmary, Edinburgh Greater awareness of sleep apnoea, both within the medical profession and the public, has resulted in a steady increase $\underline{\sigma}$ in the number of referrals to sleep laboratories. Home sleep monitoring $\overline{\bar{\sigma}}$ is an obvious method of increasing patient assessment and thus $\overparen{D}$ reducing waiting lists. We have, therefore, compared full polysomnography in our laboratory with home monitoring using the Edentrace II system (Edentec Corporation) which monitors airflow, $\vec{\circ}$ heart rate, chest wall movement and $\mathrm{O}_{2}$ saturation. Fourteen patients had laboratory polysomnography and were instructed in the use $\vec{\omega}$ of the Edentrace system for home monitoring. Staff, patient and equipment costs, together with sleep study indices, were compared. $\overrightarrow{\vec{x}}$ Two home studies failed due to machine faults, and there were two or failures due to patient connection errors. In the 10 patients with successful home studies there was no significant difference in apnoea/ hypopnoea index (AHI) per hour in bed (Edentrace mean (SE) 23 (4); polysomnography $21(4) ; p=0.4$ ) or between AHI per hour in $\overrightarrow{0}$ bed for Edentrace and AHI per hour of sleep assessed by poly- $O$

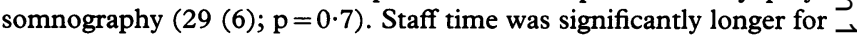
polysomnography (600 (19) minutes) than for home Edentrace (47 (3) minutes; $\mathrm{p}<0 \cdot 001$ ). Home Edentrace monitoring required two laboratory visits per patient, thus travel costs were twice that of polysomnography ( $£ 14.50 v £ 7.25 /$ patient). However travel costs $\vec{\theta}$ were limited by only using patients within a 20 mile radius of the of laboratory. The overall cost (staff, travel and equipment) of home Edentrace monitoring was $33 \%$ of that of polysomnography. In this ongoing study the portable Edentrace II system seems to be a cost effective method of home diagnosis for SAHS giving comparable $\bar{D}$ results to full polysomnography, but no data were obtained in four of 14 subjects in this ongoing study.

Nocturnal oximetry and transcutaneous $\mathrm{CO}_{2}$ in patients with cystic fibrosis

DL SMITH, DE STABLEFORTH Adult Cystic Fibrosis Unit, Birmingham Heartlands Hospital, Birmingham Nocturnal hypoxaemia is a well recognised phenomenon in cystic fibrosis (CF). In 35 patients studied $\overline{0}$ at the end of a course of hospital treatment and 22 patients studied at home we found significant nocturnal hypoxaemia (SNH, defined $?$ as a percentage of total study time with an $\mathrm{SaO}_{2}$ of $<90 \%$ or $>10 \%$ ) in $25 \%$ of patients. These patients may benefit from correction of 0 nocturnal hypoxaemia with the administration of oxygen. We have studied the characteristics of noctural $\mathrm{CO}_{2}$ traces in $18 \mathrm{CF}$ patients $\frac{7}{0}$ with moderate to severe $\mathrm{CF}$ (\%FEV $16-53$, mean 31). Transcutaneous $\mathrm{CO}_{2}$ tensions $\left(\mathrm{PtcCO}_{2}\right)$ measurements were made with a $\mathrm{N}$ TCM3 system (Radiometer, Copenhagen) previously validated against arterial samples in 10 patients studied overnight. Six of the 0 18 patients studied exhibited SNH. Mean $\mathrm{PtcCO}_{2}$ ranged from $3.75 \mathrm{~N}$ to $7 \cdot 0 \mathrm{kPa}$. Five patients had a mean $\mathrm{PtcCO}_{2}$ of $6.0 \mathrm{kPa}$ or more. The $\sigma$ maximum overnight rise in $\mathrm{PtcCO}_{2}$ ranged from 0.2 to $1.0 \mathrm{kPa}$. The pattern of overnight $\mathrm{PtcCO}_{2}$ recordings showed a basically stable trace with episodic elevations associated with simultaneous falls in $\mathrm{SaO}_{2}$. However, not all falls in $\mathrm{SaO}_{2}$ were associated with elevations in $\mathrm{PtcCO}_{2}$, suggesting that at least two different mechanisms might account for the episodes of nocturnal desaturation seen. Spirometric $\frac{1}{\mathbb{D}}$ parameters were not correlated with any measurements of $\mathrm{PtcCO}_{2} ;$ however, minimum nocturnal $\mathrm{SaO}_{2}$ was correlated with maximum $\mathrm{Q}$ $\mathrm{PtcCO}_{2}$ and maximum rise in $\mathrm{PtcCO}_{2}$ (maximum $\mathrm{PtcCO}_{2}-$ mean $\left.\mathrm{PtcCO}_{2}\right)$. Six patients with $\mathrm{SNH}$ were further studied during administration of noctural oxygen therapy at a flow rate of $21 / \mathrm{min}$. SNH was abolished in five and greatly ameliorated in the other patient. Oxygen administration resulted in an increase in mean $\mathrm{PtcCO}_{2}$ in all patients ranging from 0.4 to $1.6 \mathrm{kPa}$ (mean $0.8 \mathrm{kPa}$ )

\section{The role of CD10 in the modulation of neutrophil function}

M MIKAMI, CG LLEWELLYN-JONES, RA STOCKLEY Lung Immunobiochemical Research Laboratory, The General Hospital, Steelhouse 
Lane, Birmingham CD10/NEP (neutral endopeptidase) is a zinc metalloproteinase originally identified on acute lymphoblastic leukaemia cells and more recently found on neutrophil surface membranes where it is thought to have a modulating effect on neutrophil function (Connelly et al. Proc Natl Acad Sci USA 1985; 82:8737). We have therefore studied the role of CD10 in neutrophil chemotaxis, degranulation and superoxide generation. In order to do this we have studied the effects of the metalloproteinase inhibitor, 1,10phenanthroline and a monoclonal antibody to CD10. 1,10-phenanthroline reduced neutrophil chemotaxis to $10 \mathrm{nM}$ FMLP from a mean of $40 \cdot 7(4 \cdot 8)$ cells/field to $1 \cdot 2(0 \cdot 2)$ cells/field in a dose dependent manner $(p<0.002)$. In addition, 1,10 -phenanthroline $(0.1 \mathrm{nM})$ preincubated with cells resulted in a shift of the FMLP dose response for neutrophil chemotaxis to the left. However, 1,10-phenanthroline had only a slight inhibitory effect on neutrophil chemotaxis to IL-8 with no shift of the IL-8 dose reponse. Similarly the anti-CD10 antibody reduced the neutrophil chemotaxis to $10 \mathrm{nM}$ FMLP significantly $(p<0.04)$ in a dose dependent manner, from a control mean of $26.8(1 \cdot 8)$ to $14 \cdot 7(1 \cdot 1)$ cells/field at antibody concentration of $25 \mu \mathrm{g} / \mathrm{ml}$ and shifted the dose response to the left. The IL-8 effect was also reduced by the antibody but there was no associated shift in the dose response. Neither extracellular proteolysis nor superoxide anion release was influenced by either agent. These data suggest that the metalloproteinase, CD10 plays a minor role in the modulation of neutrophil chemotaxis to FMLP and possibly IL-8. The observed shift in the FMLP dose response suggests that CD10 may exert its modulating effect on neutrophil responses to FMLP by cleavage of FMLP peptide bonds.

\section{Effects of interleukin 8 on neutrophil function and the inter-} action with FMLP

M MIKAMI, CG LLEWELLYN-JONES, RA STOCKLEY Lung Immunobiochemical Research Laboratory, The General Hospital, Steelhouse Lane, Birmingham Interleukin 8 (IL-8) is an important inflammatory mediator which may play a role in the pathogenesis of lung diseases. However, there is some controversy regarding the effects of IL-8 on neutrophil function. Although it is known that IL-8 is a potent chemoattractant and is able to stimulate superoxide production, there is uncertainty whether it can alter neutrophil degranulation. Therefore we have designed experiments to look at the effect of IL-8 on resting neutrophils and their response to FMLP. IL-8 showed a dose response effect on chemotaxis with a maximum at a concentration of $5 \mathrm{nM}$ (mean (SE) $22.7(1.7)$ cells/field). Neutrophil degranulation was also increased from a control value of $1.93(0.31) \mu \mathrm{g} \mathrm{FN}$ to 2.85 $(0.33) \mu \mathrm{g} \mathrm{FN}$ at $10 \mathrm{nM}(\mathrm{p}<0.02)$. Finally, superoxide generation was increased from a control value of $2.79(0.37) \mathrm{nmol} / \mathrm{h}$ to 4.31 $(0.44) \mathrm{nmol} / \mathrm{h}(\mathrm{p}<0.01)$ at $10 \mathrm{nM}$. Preincubation of neutrophils with IL-8 showed a dose dependent suppression of chemotaxis to FMLP from a control mean of $18.2(4 \cdot 2)$ to $8.5(3.9)$ cells/field at $10 \mathrm{~nm}$ $(p<0.01)$. When IL-8 was used as a chemoattractant together with FMLP it showed an additive effect at suboptimal concentration of each agent. However, at optimal concentrations the chemotaxis was increased less than would be expected for each alone (IL-8 $=17 \cdot 1$ $(1 \cdot 1)$ cells/field, FMLP $=25 \cdot 0(2 \cdot 6)$ cells/field, $\mathrm{IL}-8+\mathrm{FMLP}=30 \cdot 2$ $(2 \cdot 6)$ cells/field). FMLP-induced neutrophil degranulation increased after preincubation of the cells with IL-8 $(10 \mathrm{nM})$ from a mean of 4.67 $(0.61)$ to $6.20(0.77) \mu \mathrm{g} \mathrm{FN}(\mathrm{p}<0.04)$. FMLP-induced superoxide generation also demonstrated an increase after preincubation with $5 \mathrm{nM}$ IL-8 from a control mean of $15.58(2 \cdot 79)$ to $21 \cdot 32(2 \cdot 1) \mathrm{nmol} /$ $\mathrm{h}$. This increase, $5.74(0.94) \mathrm{nmol} / \mathrm{h}$, was greater $(\mathrm{p}<0.01)$ than the effect of IL-8 treatment alone (increase $=0.84(0.32) \mathrm{nmol} / \mathrm{h}$ ), suggesting synergism. These data indicate that IL-8 is not only a potent chemoattractant for neutrophils but also that it stimulates other neutrophil functions such as degranulation and superoxide generation. Preincubation of neutrophils with IL-8 reduces the chemotactic response to FMLP but has an additive effect with FMLP as a chemoattractant and stimulant of degranulation. Finally, IL-8 acts synergistically with FMLP for superoxide generation.

Interleukin 8 may be involved in the recruitment of neutrophils into the respiratory tract of smokers and patients with chronic obstructive pulmonary disease

VM KEATINGS, PD COLLINS, DM SCOTT, TJ WILlIAMS, PJ BARNES Departments of Thoracic Medicine and Applied Pharmacology, The National Heart and Lung Institute, London It is known that patients with chronic obstructive pulmonary disease (COPD) have increased numbers of neutrophils in the airway lining fluid compared with controls. This observation has also been made in smokers with normal lung function. The mechanisms for this are unknown. The cytokine interleukin 8 (IL-8) is produced by alveolar macrophages, epithelial cells, and neutrophils. Its production is stimulated by TNF $\alpha$, interleukin 1, and neutrophil elastase. We wished to determine whether this cytokine was detectable in the airway lining fluid of patients with COPD. We induced sputum using inhaled hypertonic saline in 13 patients with COPD, 12 healthy smokers, and 16 normal nonsmoking controls. Total and differential cell counts were carried out. A proportion of the sputum was ultracentrifuged at $60000 \mathrm{~g}$ to obtain the sol phase which was assayed for IL-8 using a radioimmunoassay. Patients with COPD and healthy smokers had significantly higher IL-8 concentrations than the non-smoking controls $(1.73,0.57$ and $0.13 \mathrm{nmol}$ respectively, $\mathrm{p}=0.03$ and $\mathrm{p}=0.02$.) In the COPD group the IL-8 concentrations correlated strongly with the total neutrophil concentration $(r=0.78)$, suggesting that IL-8 and the neutrophilia are linked in this condition.

Tumour necrosis factor $\alpha$ is present in high concentrations in the airways of patients with chronic obstructive pulmonary disease

VM KEATINGS, DM SCOTT, PJ BARNES Department of Thoracic Medicine, The National Heart and Lung Institute, London It is known that patients with chronic obstructive pulmonary disease (COPD) have increased numbers of neutrophils in the airway lining fluid compared with controls. This observation has also been made in smokers with normal lung function. The mechanisms for this are unknown. The cytokine tumour necrosis factor $\alpha(\mathrm{TNF} \alpha)$ is produced by alveolar macrophages and is chemotactic for neutrophils both directly and through the induction of adhesion molecules. It has been implicated in the pathogenesis of the cellular infiltrate and the bronchial hyperresponsiveness seen in asthma. We wished to determine whether this cytokine was present in the airways of patients with COPD. We induced sputum using inhaled hypertonic saline in 13 patients with COPD, 16 patients with asthma, 12 healthy smokers, and 16 normal non-smoking controls. Total and differential cell counts were carried out. A proportion of the sputum was ultracentrifuged at $60000 \mathrm{~g}$ to obtain the sol phase which was assayed for TNF $\alpha$ using an enzyme linked immunosorbent assay. Levels of TNF $\alpha$ did not differ between healthy smokers and normal controls (concentrations 26.5 and $16.3 \mathrm{pg} / \mathrm{ml}$, respectively). Patients with COPD had significantly higher TNF $\alpha$ concentrations $(98 \cdot 1 \mathrm{pg} / \mathrm{ml})$ than the smoking and non-smoking controls $(p=0.04$ and $p=0.01)$. TNF $\alpha$ concentrations in the patients with COPD were higher than in the asthmatic group, but this did not reach significance $(p=0.07)$. These data suggest that there is a significant inflammatory response in the airways of patients with COPD and that TNF may play a significant part in this.

Contribution of neutrophils, tumour necrosis factor and glutathione to cigarette smoke-induced increased airspace permeability

XY LI, I RAHMAN, K DONALDSON, W MACNEE Respiratory Medicine Unit, Department of Medicine, University of Edinburgh; Department of Biological Sciences, Napier University, Edinburgh Increased airspace epithelial permeability (EP) is characteristic of cigarette smokers and is important in augmenting the inflammatory response in the airspaces and hence may have a role in the pathogenesis of emphysema. The purpose of this study was to investigate the mechanism of this phenomenon. We have previously shown that intratracheal instillation of cigarette smoke condensate (CSC) induces increased EP in vivo in rats and in vitro in epithelial cell monolayers associated with a disturbance in glutathione (GSH), a major lung antioxidant. In this model neutrophils account for $5-10 \%$ of the bronchoalveolar (BAL) cells. Depletion of neutrophils and macrophages by an intraperitoneal injection of anti-neutrophil antibody did not influence the EP induced by CSC. The role of tumour necrosis factor (TNF) was also investigated. Although instillation of TNF $\alpha$ increased EP in the rat lung $(0.62(0.04)$ to $1.27(0.29) \%, p<0.001) 16$ hours after instillation, only a trivial amount of TNF was detected in BAL fluid in vivo or in culture medium from BAL leucocytes obtained from CSC-treated animals. Furthermore, anti-TNF antibody did not abolish the increased EP induced by CSC. The CSC-induced increased EP was, however, associated with a profound fall in BAL GSH $(2.56(0.91)$ 
to $0.31(0 \cdot 21) \mathrm{nmol} / \mathrm{ml}, \mathrm{p}<0.001)$ and in lung GSH (869 (78) to 502 $(70) \mathrm{nmol} / \mathrm{g}$ lung ww, $\mathrm{p}<0.001$ ) associated with an increase in oxidised GSH (90 (6) to $149(85), p<0.01)$ one hour after instillation with a return to control values in lung but not in BAL six hours after instillation. These studies confirm an important role for the antioxidant glutathione in the increased epithelial permeability caused by cigarette smoke condensate.

\section{Cigarette smoke and rat lung glutathione metabolism}

I RAHMAN, XY LI, K DONALDSON, W MACNEE Respiratory Medicine Unit, Department of Medicine, University of Edinburgh; Department of Biological Sciences, Napier University, Edinburgh Cigarette smoke contains over 4700 highly electrophilic chemicals. The glutathione redox system is protective against smoke-induced injury. We studied the acute effects of cigarette smoke condensate (CSC) on glutathione (GSH) metabolism in rat lung and primary cultured type II alveolar epithelial cells. One hour after intratracheal instillation of CSC (table) and in

\begin{tabular}{lllrl}
\hline $\begin{array}{l}\text { Time } \\
\text { (hours) }\end{array}$ & GSH & $\begin{array}{l}\text { GSH conjygates } \\
\text { (nmollg lung wet wt) }\end{array}$ & GSSG & $\begin{array}{l}\text { PrSSG (nmoll } \\
\text { mg protein) }\end{array}$ \\
\hline 0 & $780(32)$ & $22(8)$ & $94(10)$ & $32(6)$ \\
1 & $510(70)^{* *}$ & $72(7)^{* *}$ & $105(10)$ & $28(2)$ \\
6 & $775(60)$ & $44(8)^{*}$ & $65(4)$ & $27(2)$ \\
24 & $770(45)$ & $32(8)$ & $93(5)$ & $29(2)$ \\
\hline
\end{tabular}

${ }^{*} \mathrm{p}<0.05,{ }^{* *} \mathrm{p}<0.001$. Values are mean (SE).

vitro exposure of epithelial cells GSH diminished, without elevation of oxidised glutathione (GSSG) or protein-GSH mixed disulphides (PrSSG). However, the depletion of GSH was associated with rapid elevation of GSH conjugates unrelated to the action of glutathioneS-transferase. Activities of $\gamma$-glutamylcysteine synthetase, glutathione peroxidase, and glucose-6-phosphate dehydrogenase were significantly decreased after one hour, returning to normal levels after six hours exposure to CSC without change in glutathione-S-transferase and glutathione reductase activities. Thus CSC exposure causes transient depletion of GSH by the formation of GSH conjugates, in association with inhibition of GSH synthesis and other redox enzymes. These results are relevant to the changes in glutathione metabolism in smoker's lungs and the associated airways injury. [Supported by the Tobacco Products Research Trust and The Norman Salvesen Emphysema Research Trust.]

Nitration of tyrosine by gas phase cigarette smoke: a mechanism of biomolecular damage by exposure to excess nitric oxide?

JP EISERICH, V VOSSEN, CA O'NEILL, B HALLIWELL, CE CROSS, A VAN DER VLIET Division of Pulmonary/Critical Care Medicine, University of California, Davis, California, USA Nitric oxide (NO) is a chemical species of considerable recent interest, both because of its multiple physiological roles (ranging from blood pressure modulation to neurotransmission) and because of its toxic effects when generated in excess. NO is present in cigarette smoke (CS) at up to $500 \mathrm{ppm}$, and probably represents one of the greatest exogenous sources of 'NO to which humans are exposed. We have previously shown that CS induces depletion of plasma aqueous phase antioxidants, extensive protein modification and small amounts of lipid peroxidation. Oxidative damage to proteins by CS, as measured by protein carbonyl formation, was found to be primarily due to reactions of $\alpha, \beta$-unsaturated aldehyes with protein $-\mathrm{SH}$ and $-\mathrm{NH}_{2}$ groups. Free radical oxidative damage to proteins by CS thus has not been directly observed. In the present investigation we have explored the possibility that nitrogen oxides present in CS may modify proteins in the respiratory tract. To test this hypothesis phosphate buffered solutions of $N$-acetyltyrosine $(1 \mathrm{mM})$ were exposed to approximately $50 \mathrm{ml}$ puffs of gas phase CS and incubated at $37^{\circ} \mathrm{C}$ for various periods of time (1-20 min) before the next puff of CS was administered. After a total of 18 puffs of gas phase CS the samples were hydrolysed and the concentrations of 3-nitrotyrosine were found to range from 0.3 to $17 \cdot 1 \mu \mathrm{M}$ for incubation times ranging from $1-20$ minutes, respectively. Dityrosine, the dimerisation product of tyrosyl radicals, was also formed upon CS exposure, but at much lower levels than 3-nitrotyrosine. Both products were also formed in solutions of albumin exposed to CS. Glutathione, ascorbic acid and uric acid, important antioxidants found in the respiratory tract lining fluids, decreased the CS-induced formation of both 3-nitrotyrosine and dityrosine. We suggest that nitrogen oxides in CS modify proteins in the respiratory. tract and may contribute to CS toxicity by inactivating enzymes and $\overrightarrow{\vec{F}}$ membrane transport proteins, and interfering with cell signalling $\stackrel{5}{\rightarrow}$ pathways involving tyrosine phosphorylation. These results are also? relevant to other situations in which excess exposure to $\mathrm{NO}$ causes toxicity in humans.

Effectiveness of low molecular weight thiols in protecting epithelial lining fluids from ozone-induced damage

A VAN DER VLIET, CA O'NEILl, JP EISERICH, B HALLIWELl, CE CROss Department of Internal Medicine, UC Davis Medical Center, $\rightleftharpoons$ Sacramento, California, USA Ozone $\left(\mathrm{O}_{3}\right)$ is a powerful oxidant in $\stackrel{\times}{\times}$ photochemical smog, and the first biological matrix that will interacto with $\mathrm{O}_{3}$ upon inhalation are the epithelial lining fluids (ELFs). Because? of difficulties in obtaining ELFs we used blood plasma as a surrogate $\vec{\omega}$ extracellular fluid to study interactions of $\mathrm{O}_{3}$ with ELF constituents. Plasma was exposed to $\mathrm{O}_{3}(2-16 \mathrm{ppm})$ for two hours. Upon exposure to $\mathrm{O}_{3}$ plasma levels of ascorbate and urate declined rapidly, some 5 depletion was observed in protein thiols, and increaseed levels of protein carbonyls were detected, indicative of (oxidative) protein $\frac{7}{0}$ modification. At concentrations up to $8 \mathrm{ppm} \mathrm{O}_{3}$ did not significantly affect plasma levels of $\alpha$-tocopherol or ubiquinol, nor were lipid $\vec{\bullet}$ hydroperoxides detected. Both GSH $(1 \mathrm{mM})$ and dihydrolipoic acide (DHLA) did not afford significant protection against antioxidant ${ }^{\text {. }}$ depletion or protein carbonyl formation by $\mathrm{O}_{3}$. A slight decrease in ascorbate depletion was observed in the presence of DHLA, possibly because it inhibits autooxidation of ascorbate. When thiols such as GSH or DHLA are added to plasma, rapid oxidation of the added@ thiol occurs. For instance, when $1 \mathrm{mM}$ GSH was added to plasma only $50 \mu \mathrm{M}$ GSH could be detected after two hours. With both thiols it was observed that reduced protein thiol levels were markedlyö increased, indicating interaction of the added thiols with mixed 3 disulfides on proteins. The studies presented here indicate that low molecular weight thiols such as GSH or DHLA are ineffective in preventing reactions of inhaled $\mathrm{O}_{3}$ with ELF constituents. However, these thiols may have protective effects against secondary products 0 from interactions of $\mathrm{O}_{3}$ with ELF constituents such as hydrogen $\underset{\times}{\mathbb{N}}$ peroxide and aldehydes.

Ozone-induced export of glutathione and epithelial permeability in vitro is associated with cytoskeletal disruption

D MORRISON, A LANGRIDGE, XY LI, I RAHMAN, W MACNEE Unit of Respiratory Medicine, Department of Medicine, Royal Infirmary, Edinburgh Epithelial permeability is increased in cigarette smokers $\mathrm{N}$ and following ozone exposure (Jones. Lancet 1980; Kehrl. Am Rev Respir Dis 1987). The mechanism is unknown. Both have been shown to deplete intracellular reduced glutathione (GSH), an importantc thiol antioxidant. Thiol groups may have an important role in the function of the cytoskeleton. We therefore studied the effect of in vitro exposure to 1000 parts per billion (ppb) ozone for one hour on GSH levels, epithelial permeability and the cytoskeleton in monolayers of A549 type II alveolar epithelial cells. Cells were exposed in air/ $5 \% \mathrm{CO}_{2}$ at $37^{\circ} \mathrm{C}$. There was a trend for intracellular $\mathrm{GSH}$ and its oxidised form GSSG to fall (GSH: control, mean (SE) $6.0(0.9) \mathrm{nmol} / \stackrel{\mathbb{\Phi}}{\mathbb{D}}$ $10^{6}$ cells, ozone, $5 \cdot 1(0.9)$; GSSG: control, $0.13(0.05)$, ozone $0.08 \frac{\varrho}{9}$ $(0.04), n=6)$. In addition, glutathione increased in the medium (control, $0.12(0.03)$, ozone $0.28(0.04), \mathrm{p}<0.001)$. Epithelial per-ত meability to ${ }^{125} \mathrm{I}$-albumin increased from $0.12(0.02) \%$ to 0.17 $(0.03) \%, p<0.001$, following ozone $(n=5)$. The percentage of cellso showing the normal distribution of actin decreased from 60.4 (3.9) to $22 \cdot 7(1 \cdot 9), p<0.001(n=3)$. In conclusion, exposure of A549 cellsô to ozone $1000 \mathrm{ppb}$ for one hour produced export of glutathione and? increased epithelial permeability in association with disruption of the cytoskeleton. [Supported by the British Lung Foundation.] 